\title{
SIMULATION OF AIR FLOW AND DROP TRAJECTORIES IN THE NASA GLENN ICING RESEARCH TUNNEL
}

\author{
A Thesis \\ Presented to \\ the faculty of the School of Engineering and Applied Science \\ University of Virginia \\ In Partial Fulfillment \\ of the requirements for the Degree \\ Master of Science Mechanical and Aerospace Engineering \\ by \\ Kevin McAllister Clark
}

May 2012 


\section{APPROVAL SHEET}

This thesis is submitted impartial fulfillment of the requirements for the degree of

Master of Science Mechanical and Aerospace Engineering

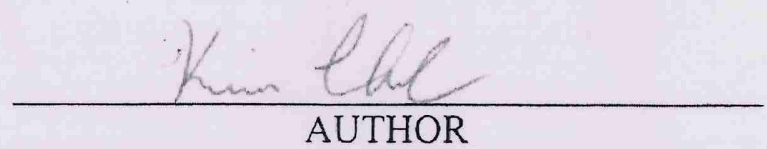

The thesis has been read and approved by the examining committee

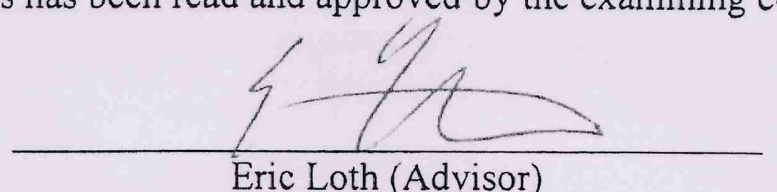

Eric Loth (Advisor)

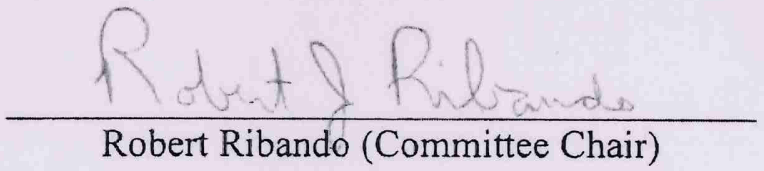

$\frac{2+21}{\text { Houston Wood }}$

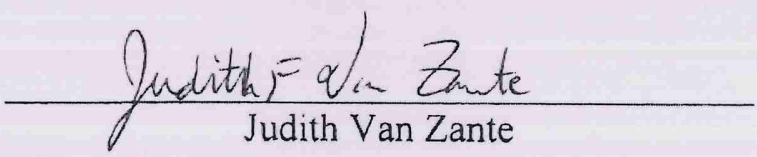

Accepted for the School of Engineering and Applied Science:

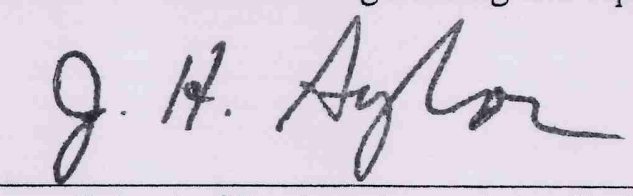

Dean, School of Engineering and Applied Science

May 2012 


\begin{abstract}
The NASA Glenn Icing Research Tunnel (IRT) was constructed in 1944 to replicate aircraft icing conditions seen during flight in a controlled environment for analysis and certifications. The tunnel is periodically updated and modified to improve air flow quality and droplet distribution uniformity. In the first portion of this study (Part I), Computational Fluid Dynamics (CFD) was applied to three IRT geometries corresponding to configurations in 2000, 2009 and 2012. The simulations employed three-dimensional Reynolds-Averaged Navier-Stokes (RANS) modeling for the turbulent air flow combined with Lagrangian trajectories for the water droplets. These trajectories diffuse stochastically based on the turbulent kinetic energy of the air flow. Their distribution is important for predicting and understanding Liquid Water Content (LWC) uniformity.
\end{abstract}

The Year 2000 tunnel configuration was simulated from the spray bars to the test section and the results indicated that the RANS model predicted reasonable test section conditions. The 2009 tunnel configuration also was initiated just upstream of the spray bars but included vertical struts that were installed to increase LWC uniformity as well as multiple Mod-1 air jets implemented using embedded velocity profiles. These changes were found to increase the turbulent kinetic energy throughout the IRT. The 2012 tunnel configuration simulation included a new heat exchanger installed in 2011 as well as the ensuing Corner D which is upstream of the spray bars. The heat exchanger was simulated using a two-dimensional RANS model to provide an inlet boundary condition for the 3-D tunnel flow simulation in to Corner D. The results indicated an increase in turbulent kinetic energy from the 2009 tunnel configuration, especially near the inner wall. A transfer map was developed to show the droplet locations in the test section for specific 
water nozzles and was compared to maps developed experimentally. The result indicated good qualitative agreement, but under-predicted the droplet diffusion. Future studies should investigate the 2012 calibrated nozzle positions and the unsteadiness emanating from the spray bars and vertical struts (as discussed in Part II) to improve modeling fidelity and investigate opportunities to improve the LWC uniformity at the test section. Similarly, modifications to the spray bar and tunnel walls should also be considered to improve uniformity.

For the second portion of this study (Part II), a hybrid RANS and Large Eddy Simulation (LES) model was utilized to capture the unsteady phenomenon, e.g. wake shredding and flow recirculation, observed in jets released from certain water nozzles in the IRT. Since the RANS/LES model is computationally expensive, the domain was restricted to the region near a spray bar and its spray nozzles. A new boundary condition method was developed to translate the unsteady near-field air jet flow generated by a nozzle into a larger domain (far-field). The technique was denoted the Recorded Interface Boundary Condition and was found to reasonably reproduce the mean and turbulent velocities in the far-field without requiring the small time-steps and high resolution domain associated with the near-field. A single spray bar without any vertical struts or active nozzles was simulated with the RANS/LES model to study the unsteady flow in its wake. Results indicated high vortex shedding and flow separation which create a large unsteady wake downstream with greater turbulence intensity and wake spread than that predicted with the RANS description. The RANS/LES single spray bar simulation and new methodology for modeling jet flows form a foundation which spray bars with vertical struts and active water nozzles should be integrated. The RANS/LES model is 
recommended to be incorporated into the full IRT model and is expected to improve the fidelity of the droplet trajectories and LWC predictions. 


\section{Acknowledgements}

I want to thank my adviser Professor Loth for bringing me into the project. I would not have been able to have accomplished so much without his guidance and encouragement. I want to also thank Albert Lee, Yong Yeong, and Tyler Gillen for their assistance in getting me setup at University of Virginia and the software needed for my research. Thank you to my undergraduate assistant, Michael Malinowski, for his assistance and work on the project. I would also like to thank the many members of Loth's research group and UVA engineering graduate students for their support and friendship during my studies.

This research was funded by the Icing Branch of the NASA Glenn Research Center and was much appreciated. I especially want to thank Judy Van Zante, Robert Ide, Laura Steen, and Colin Bidwell. Their guidance and direction on the project was extremely helpful as well as their wiliness to share their knowledge of the Icing Research Tunnel.

Finally I want to thank my family for providing emotional and financial support during the course of my education. I want to thank my mother for giving me the best guidance she could for any situation and my brother, Corey, for the advice and competiveness that only an older brother could provide. 


\section{Table of Contents}

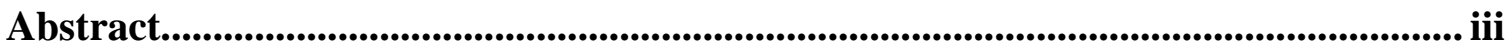

Acknowledgements.......................................................................................................................... vi

List of Figures....................................................................................................................................

List of Tables................................................................................................................................... xi

List of Symbols............................................................................................................................. xii

Part I. RANS Simulations of the NASA Glenn Icing Research Tunnel........................ 1

Chapter 1. Introduction............................................................................................. 1

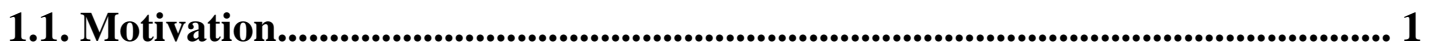

1.2. NASA Glenn IRT............................................................................................... 3

1.3. Previous NASA IRT Simulations............................................................................ 10

1.4. Objectives.............................................................................................................. 13

Chapter 2. Air Flow Simulations.............................................................................................. 15

2.1. Methods.................................................................................................................... 15

2.2. Quarter-Tunnel for 2000 and 2009 IRT Configurations................................. 26

2.3. 2-D Simulations of the 2012 Heat Exchanger...................................................... 35

2.4. Full Tunnel with Turning Vanes............................................................................. 40

Chapter 3. Liquid Water Concentration and Droplet Trajectories.......................... 53

3.1. Methods...................................................................................................................... 53

3.2. Droplet Trajectories and Transfer Map.............................................................. 57

Chapter 4. Conculsions........................................................................................................... 67

Part II. Hybrid RANS/LES Simulations of the Spray Bar Region............................... 70

Chapter 5. Introduction........................................................................................................ 70

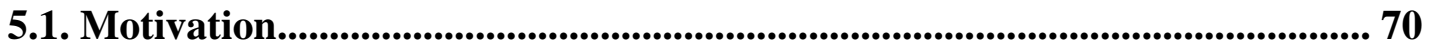

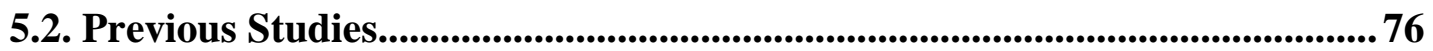

5.3. Objectives.................................................................................................................... 79

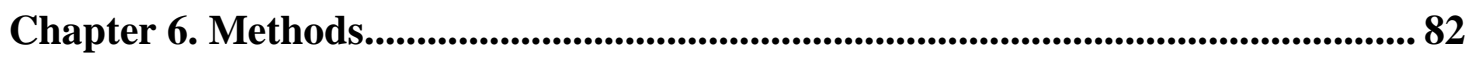

6.1. Hybrid RANS/LES Numerical Method............................................................82

6.2. Cylinder Wake Validation.................................................................................... 85

Chapter 7. New Coupling Method of Near-field and Far-Field for Jet Flows........90

7.1. Single Jet Computational Domain...................................................................... 90

7.2. Jet Interface Coupling...............................................................................................93

7.3. Results and Comparisons to Previous Studies..................................................... 96

Chapter 8. Single Spray Bar Hybrid RANS/LES Simulation................................... 109

8.1. Computational Domain......................................................................................... 109

8.2: Single Spray Bar Results........................................................................................... 110

Chapter 9. Summary and Recommendations for Future Work............................ 115

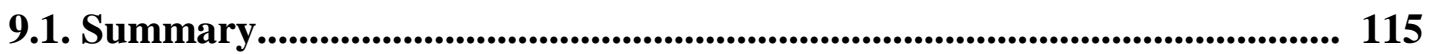

9.2. Recommendations for Future Work..................................................................... 116 


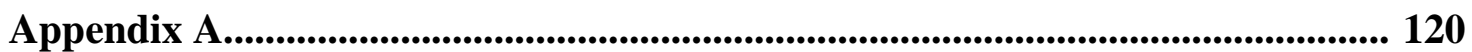

Appendix B...................................................................................................................... 124

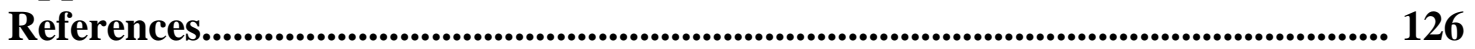




\section{List of Figures}

Figure

Figure 1.1.1. Ice accumulation on a model within the NASA Glenn IRT .................2

Figure 1.2.1. Schematic of the NASA IRT ...........................................

Figure 1.2.2. Photograph of the NASA IRT fan.........................................

Figure 1.2.3. Side view drawing of 2012 heat exchanger................................

Figure 1.2.4. Photograph of exit of 2012 heat exchanger..................................6

Figure 1.2.5. Photograph of Corner $\mathrm{D}$ with its turning vanes...........................

Figure 1.2.6. Photograph of spray bars and vertical struts............................. 8

Figure 1.2.7. Close-up picture of the spray bar's trailing edge........................................

Figure 1.2.8. Photograph of contraction upstream of the test section........................

Figure 1.2.9. Test grid used for IRT calibrations...............................................10

Figure 1.3.1. Velocity contour from simulation by Bhargava.......................................12

Figure 1.3.2. LWC plots from simulations by Bhargava.........................................12

Figure 2.1.1. Simple wake comparison between Menter SST model and experiment......18

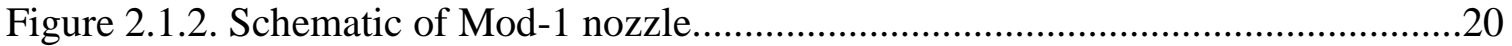

Figure 2.1.3. Axisymmetric computational mesh for single air jet flow...........................21

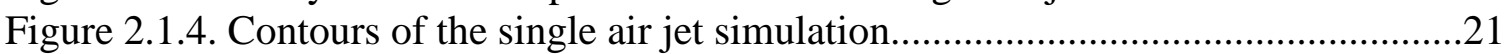

Figure 2.1.5. Contours of the single air jet simulation using a 4" profile..........................23

Figure 2.1.6. Centerline comparison of the single air jet.................................................24

Figure 2.1.7. Radial comparison at $20 \mathrm{~cm}$ for the single air jet.......................................25

Figure 2.2.1. Computational domain for 2000 IRT configuration....................................26

Figure 2.2.2. Side view contours for 2000 IRT configuration.........................................27

Figure 2.2.3. Test section turbulent kinetic energy for 2000 IRT configuration................29

Figure 2.2.4. Side view contours for 2009 IRT configuration..........................................30

Figure 2.2.5. Turbulent kinetic energy slices along 2009 IRT configuration......................31

Figure 2.2.6. Test section turbulent kinetic energy for 2009 IRT configuration.................32

Figure 2.2.7. Nozzle map for Mod-1 nozzle for 2009 IRT................................................33

Figure 2.2.8. Velocity contour showing nozzle locations for 2009 IRT simulation...........33

Figure 2.2.9. Turbulent kinetic energy slices along 2009 IRT configuration with jets.....34

Figure 2.2.10. Test section turbulence for 2009 IRT configuration with jets.....................35

Figure 2.3.1. Outline of 2-D 2012 heat exchanger simulation .........................................36

Figure 2.3.2. Detailed view of computational grid around the pipes ...............................37

Figure 2.3.3. Velocity contour of the 2-D 2012 heat exchanger simulation......................39

Figure 2.3.4. Photograph of the exit of the 2012 heat exchanger......................................40

Figure 2.4.1. Top down view of the computational grid for Corner D simulation............42

Figure 2.4.2. Detailed view of the computational grid around turning vanes ..................42

Figure 2.4.3. Inlet conditions for Corner D simulation....................................43

Figure 2.4.4. Contour slices along Corner D ..........................................4

Figure 2.4.5. Contour of outlet conditions from Corner D...............................45

Figure 2.4.6. Yaw and pitch comparison between experiments and predictions................47

Figure 2.4.7. Turbulent kinetic energy for the 2012 IRT configuration $\quad . . \ldots \ldots \ldots \ldots \ldots \ldots . . . . . . .48$

Figure 2.4.8. Turbulent length scale for the 2012 IRT configuration ........................49

Figure 2.4.9. Turbulent kinetic energy slices along the 2012 IRT configuration...............50

Figure 2.4.10. Normalized velocity fluctuations slices for the 2012 IRT configuration...51

Figure 2.4.11. Test section turbulence for 2012 IRT configuration.........................52 
Figure 3.1.1. Radial profile of the particle velocities at $10 \mathrm{~cm}$ of the nozzle...............55

Figure 3.1.2. Centerline air flow velocity for single air jet....................................56

Figure 3.1.3. Droplet velocities at 20 and $30 \mathrm{~cm}$ downstream of the nozzle....................57

Figure 3.2.1. Droplet diameter sizes using a Rosin-Rammler fit...................................58

Figure 3.2.2. Velocity contour 4" downstream of the IRT spray bars............................59

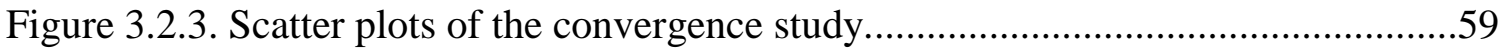

Figure 3.2.4. Concentration plots of the convergence study......................................60

Figure 3.2.5. Velocity contour 4" downstream of the spray bars during calibrations........61

Figure 3.2.6. Droplet locations for a single row of nozzles.........................................62

Figure 3.2.7. Droplet locations for a single column of nozzles..................................62

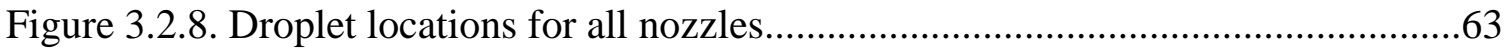

Figure 3.2.9. Transfer map for all rows and columns of nozzles...................................65

Figure 5.1.1. Snapshot of an IRT video during an experiment.....................................72

Figure 5.1.2. Time sequence of two nozzles during the IRT video................................72

Figure 5.1.3. Nozzle map showing the "dancing" intensities........................................73

Figure 5.1.4. Number of nozzles at the three "dancing" intensities for the three possible

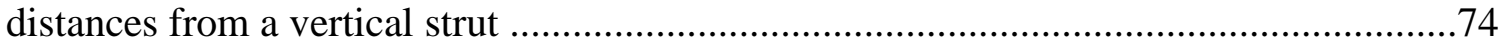

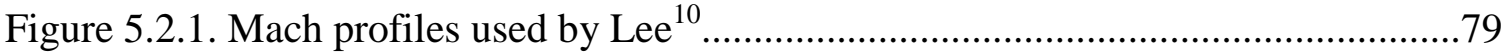

Figure 6.2.1. Computational grid for cylinder wake validation.....................................79

Figure 6.2.2. Spectral density in the wake behind a cylinder.....................................88

Figure 6.2.3. Strouhal numbers for multiple cylinder studies.......................................89

Figure 7.1.1. Computational domain for full-field single jet simulation.......................91

Figure 7.1.2. Schematic of near,far, and full-field domains.....................................99

Figure 7.2.1. Time graph displaying the two types of RIBC profiles............................96

Figure 7.3.1. Instantaneous Mach number for full-field jet simulation...........................97

Figure 7.3.2. Centerline velocity for full-field jet simulation and experiment.................98

Figure 7.3.3. Instantaneous Mach number for far-field jet simulation using a RIBC.......99

Figure 7.3.4. Time sequence of temperature contours using the two versions of RIBC.101

Figure 7.3.5. Centerline velocity for full-field and far-field jet simulations..................103

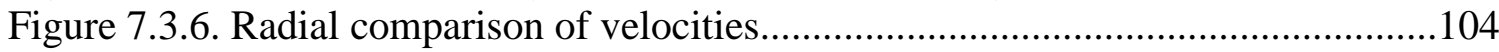

Figure 7.3.7. Radial comparison of turbulent kinetic energy.....................................105

Figure 7.3.8. Radial comparison of velocity fluctuations........................................... 108

Figure 8.1.1. Computational domain of single spray bar simulation.............................109

Figure 8.2.1. Instantaneous contours of single spray bar simulation............................111

Figure 8.2.2. Velocity contours for RANS and hybrid RANS/LES single spray bar .....112

Figure 8.2.3. Turbulent kinetic energy contours for single spray bar using RANS and

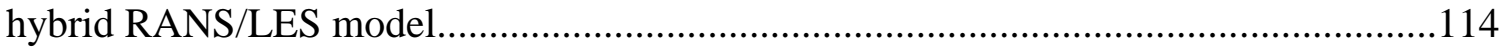

Figure 9.2.1. Examples of spray bar modification...................................................118

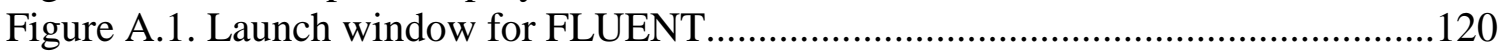

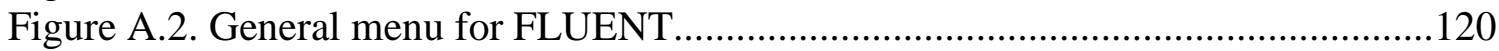

Figure A.3. The profile screen to read and write flow profiles...................................121

Figure A.4. The boundary conditions menu and window for velocity inlet boundary....121

Figure A.5. The methods menu...................................................................... 122

Figure A.6. The models menu to turn on the discrete phase model..............................122

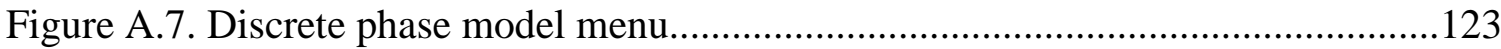

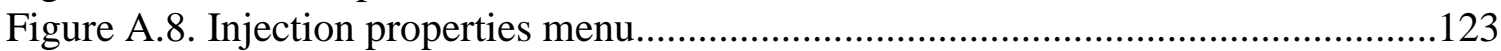




\section{List of Tables}

Table Page \#

Table 2.2.1. Test section velocity fluctuation comparison for 2000 IRT configuration....29

Table 2.2.2. Test section velocity fluctuation comparison for 2009 IRT configuration.....35

Table 2.4.1. Test section average turbulent kinetic energy ……......................................52

Table 7.1.1. Information of the computational demand for each LES simulation.............93 


\section{List of Symbols}

$\begin{array}{ll}a & =\text { speed of sound } \\ \partial & =\text { partial derivative } \\ d & =\text { droplet diameter } \\ D & =\text { cylinder diameter } \\ e & =\text { energy } \\ g & =\text { gravity } \\ G & =\text { filter function } \\ F & =\text { frequency } \\ F & =\text { fluid forces } \\ F_{1} & =\text { blending function } \\ k & =\text { turbulent kinetic energy } \\ l & =\text { turbulent length scale } \\ m & =\text { mass } \\ M & =\text { Mach number } \\ p & =\text { pressure } \\ q & =\text { arbitrary variable } \\ r & =\text { radial distance } \\ R e & =\text { Reynolds number } \\ S t & =\text { Strouhal number } \\ t & =\text { time } \\ T & =\text { temperature } \\ T_{\text {far }} & =\text { integration period for far-field } \\ T_{n e a r} & =\text { integration period for near-field } \\ u & =\text { velocity } \\ v & =\text { particle velocity } \\ W e & =\text { Weber number } \\ x & =\text { streamwise distance } \\ y & =\text { vertical distance } \\ y^{+} & =\text {dimensionless wall distance } \\ z & =\text { spanwise distance } \\ & \end{array}$

\section{Greek and Other Symbols}

$\begin{array}{ll}\alpha & =\text { water concentration } \\ \Delta & =\text { grid length } \\ \Delta t & =\text { time-step } \\ \varepsilon & =\text { dissipation rate } \\ \kappa & =\text { thermal conductivity } \\ \Lambda & =\text { turbulent length scale } \\ \mu & =\text { dynamic viscosity } \\ \nu & =\text { viscosity } \\ \rho & =\text { density }\end{array}$




$$
\begin{array}{ll}
\sigma & =\text { surface tension } \\
\tau & =\text { viscous stress tensor } \\
\tau^{s} & =\text { sub-grid scale Reynolds stress tensor } \\
\phi & =\text { arbitrary constant } \\
\omega & =\text { specific dissipation rate } \\
\zeta & =\text { random number } \\
\Omega & =\text { vorticity } \\
\xi & =\text { clipping function } \\
\nabla & =\text { divergence }
\end{array}
$$

\section{Subscripts}
( ) avg = average
( ) far $=$ far-field
( $)_{\text {near }}=$ near-field
()$_{r m s}=$ root mean square
( ) $)_{\text {res }}=$ residual
( $)_{r r} \quad=$ Rosin-Rammler
( ) sub $_{\text {sub-grid }}$
( ) $x=$ streamwise direction
( ) $y=$ vertical direction
()$_{z} \quad=$ spanwise direction 


\section{Part I. RANS Simulations of the NASA Glenn Icing Research Tunnel}

\section{Chapter 1. Introduction}

\subsection{Motivation}

Accretion of ice on aircraft surfaces has been a cause for concern since the beginning of human aviation. Ice buildup causes a multitude of issues which negatively affect aircraft performance, e.g. additional weight, damage to control surfaces, and disturbing the flow field aerodynamics. Disturbing the aerodynamics can have enormous effects on the lift and drag forces on an aircraft and may even result in flow separation on a wing causing dangerous stalling conditions. Since icing conditions are common in the lower part of the atmosphere, aircraft frequently accumulate ice during landing and takeoff. Such conditions have led to numerous incidents that have caused many casualties. Accidents caused by ice accretion has decreased in recent years due to numerous studies that have led to new innovations and de-icing techniques. However, the issue still remains and more studies are required to learn how to handle icing properly and efficiently.

Understanding the physics and effects of ice buildup requires performing experiments in an icing environment that replicates conditions seen during flight. However, flying an aircraft in the proper icing conditions relies on unpredictable weather conditions and is extremely dangerous. Icing Research Tunnels (IRT) were developed to reproduce the icing conditions on the ground in a safe and controlled environment. These tunnels are specialized wind tunnels that reduce the moving air flow's temperature and then proceed to spray water droplets into the flow field. Spraying water droplets into the high velocity air flow creates a fast moving cloud of super-cooled water droplets. Ideally, the conditions at a test section would match natural icing conditions seen during flight, 
allowing for analysis of the ice buildup on different test models. An example of ice accumulation on a model in an IRT can be seen in Fig. 1.1.1. Different design models can be placed within an IRT to replicate different aircraft components providing a safer alternative to in-flight testing.

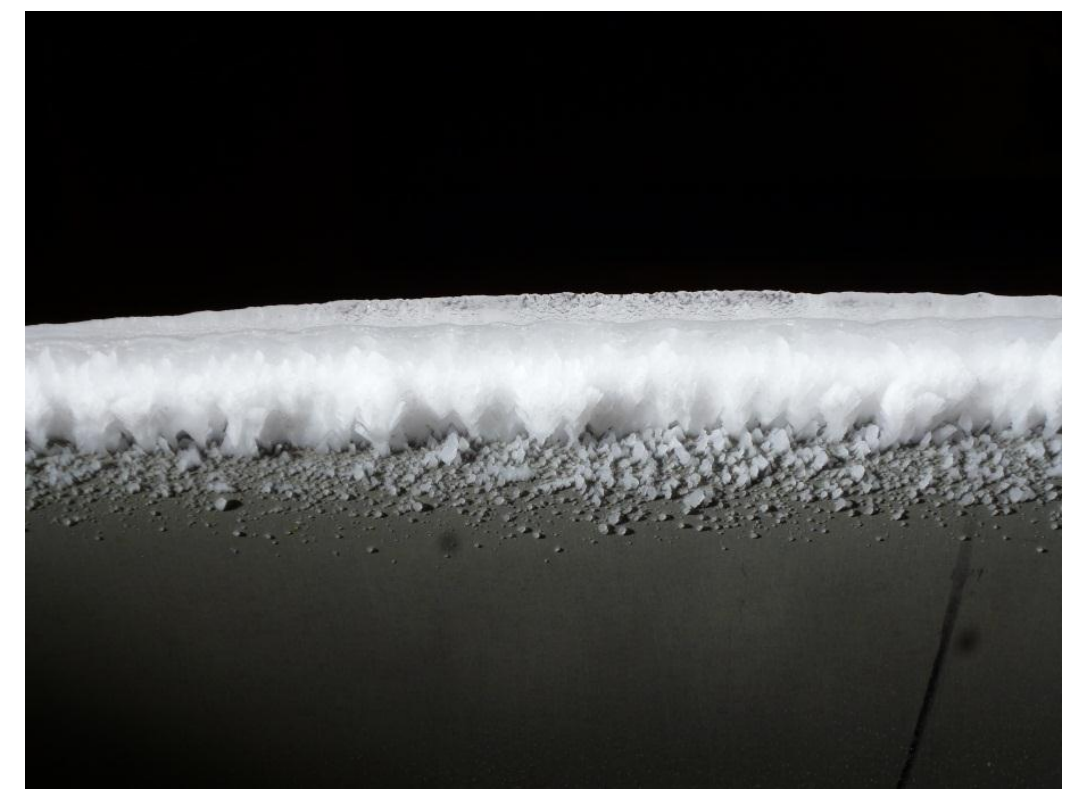

Figure 1.1.1. Ice accumulation on a leading edge of a model within the NASA Glenn IRT.

The NASA Glenn Icing Research Tunnel is one of the oldest and largest running closed loop IRTs in the world. Located in Cleveland, Ohio it was built in 1944 to study icing effects on piston and propeller driven aircraft after World War II. It is described as a closed loop tunnel because the airflow is recirculated back towards the test section instead of reintroducing new airflow (i.e. open loop). Multiple modifications have been performed on the tunnel over the years to replace aging components and incorporate new designs to improve testing conditions. However, the IRT has to be recalibrated after any tunnel modification. Recalibration is an expensive and time consuming process that involves adjusting the location of the nozzles releasing the super-cooled water droplets to 
produce the most ideal cloud. The super-cooled water cloud needs to be as large as possible while keeping uniform liquid water content (LWC) at the tunnel test section to replicate natural icing conditions.

Recalibration requires an extended amount of time because the flow physics generated by the new tunnel components are not clearly defined and their droplet trajectories are complex and asymmetric in the test section. Selecting the set of active water nozzle locations is based on an experimental testing method, which employs transfer maps followed by a series of iterations. An optimal solution is difficult to achieve since the LWC varies as a function of tunnel speeds and non-linearly with nozzle locations. Computational fluid dynamics (CFD) that solve the governing equations for a flow field have historically been used for assuming a simplified IRT geometry with uniform flow entering the spray bars as well as vertical and horizontal symmetry so that only a quarter of the tunnel air flow is simulated. Recent advances in computer processing power have allowed for more complex three-dimensional flows to be simulated. Utilizing computer simulations to model the detailed flow within the NASA Glenn IRT will allow for greater understanding of its flow physics. Knowing the behavior of certain aspects of the tunnel may allow for improved estimates during calibrations, reducing cost and time.

\subsection{NASA Glenn IRT}

A schematic of the NASA Glenn IRT after a new heat exchanger was installed in 1999 can be seen in Fig. 1.2.1. This plan view of the closed loop tunnel illustrates the locations of the different tunnel components. At the top of the schematic (between Corner $\mathrm{B}$ and Corner $\mathrm{C}$ ) is the fan that powers the tunnel and moves the air flow. Figure 1.2.2 is 
a photograph looking downstream to the fan. People may be seen walking past the fan and provide a reference to its size. After the fan, the flow moves towards Corner $\mathrm{C}$ and the heat exchanger immediately downstream of Corner C.

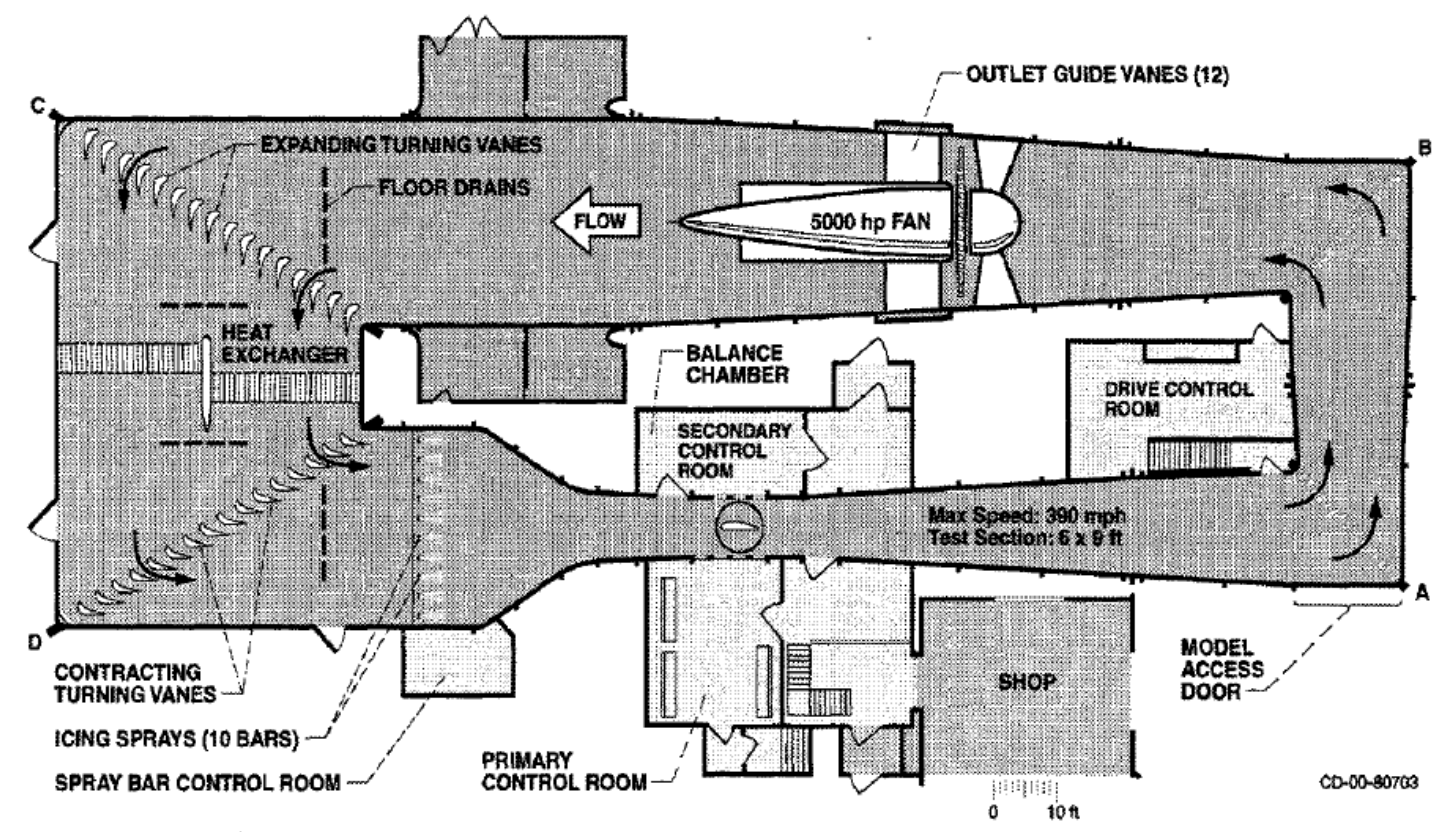

Figure 1.2.1. Schematic of the NASA IRT configuration after the 1999 modifications ${ }^{1}$.

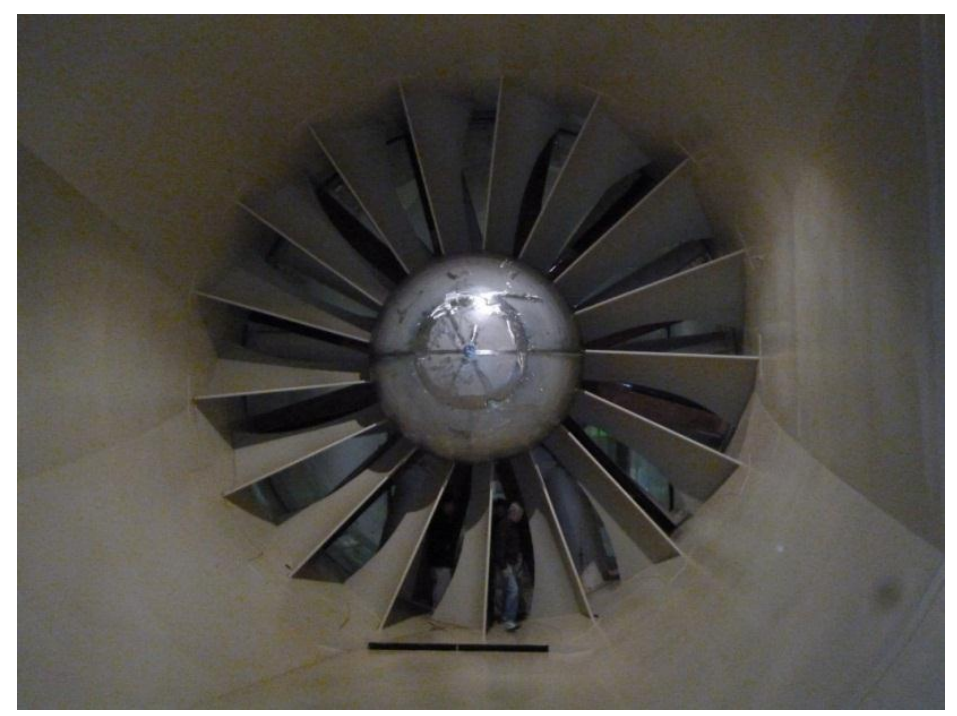

Figure 1.2.2. Photograph looking downstream to the fan that moves the air flow in the NASA Glenn IRT. 
The tunnel between Corner C and Corner D was widened to $49 \mathrm{ft}$. and a height of 26 ft. in 1999 to fit a new heat exchanger. A new heat exchanger was installed in 2012 that kept the same tunnel dimensions as the 1999 heat exchanger. Figure 1.2.3 is a side view drawing of the 2012 heat exchanger whereby the air flow moves from the left to the right. The heat exchanger is comprised of six different horizontal sections connected by splitter plates and stacked vertically from the tunnel floor to its ceiling. Figure 1.2.4 is a close-up photograph of the exit of one section of the heat exchanger. As illustrated in Fig. 1.2.3 and Fig. 1.2.4 the rows of pipes in the heat exchanger sections are arranged in an approximately 30 degree angles from the tunnel floor and into the incoming air flow. This differs from the more uniform vertical spacing seen in the 1999 heat exchanger. Exiting the heat exchanger, the flow moves into Corner D with its turning vanes.

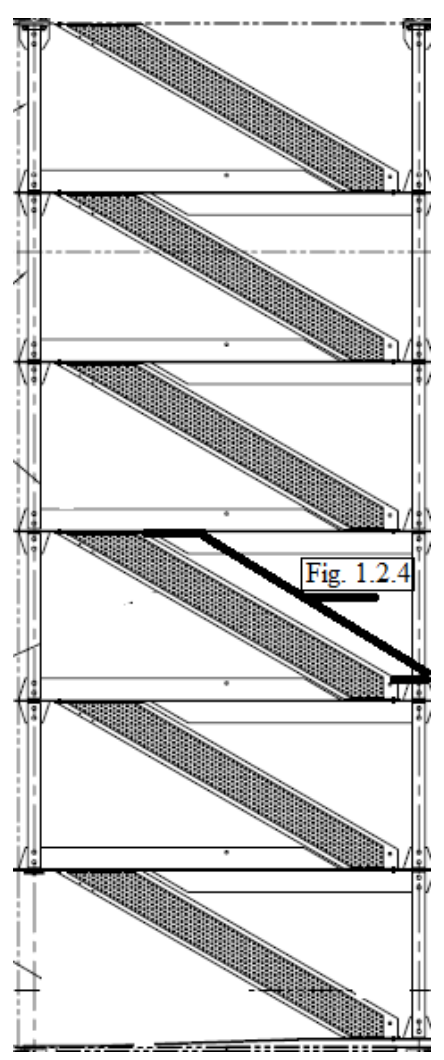

Figure 1.2.3. Side view drawing of the 2012 heat exchanger whereby the air flow moves from the left to the right. The view for Fig. 1.2.4 is shown by the black lines. 


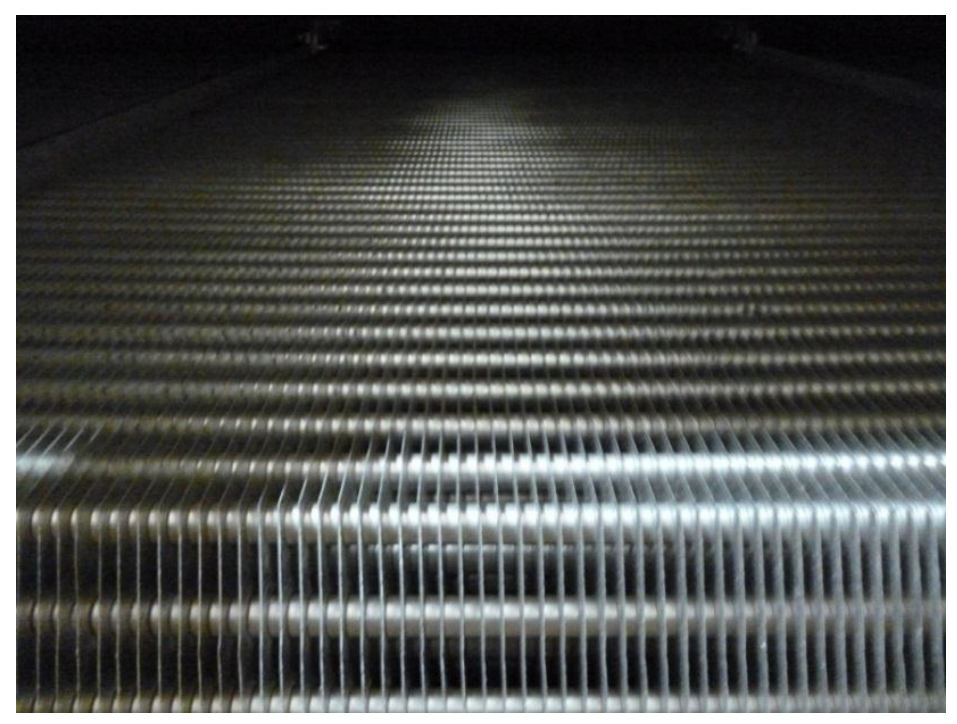

Figure 1.2.4. Close-up photograph of one section of the heat exchanger looking upstream (i.e. the photographer is standing downstream of the heat exchanger and is facing upstream). The heat exchanger includes six of these section stacked vertically in the tunnel shown in Fig. 1.2.3.

Figure 1.2.5 is a photograph of the turning vanes on Corner D with people standing in the background. Turning vanes are the key internal component of the corner and ensure that the flow direction smoothly transitions around the tunnel's sharp corner. After the corner, the width of the tunnel converts back to its original width before the 1999 heat exchanger of $29 \mathrm{ft}$. Because of this change in the tunnel's width, the turning vanes are designed to converge to compensate for the changing area and resulting increase in velocity. 


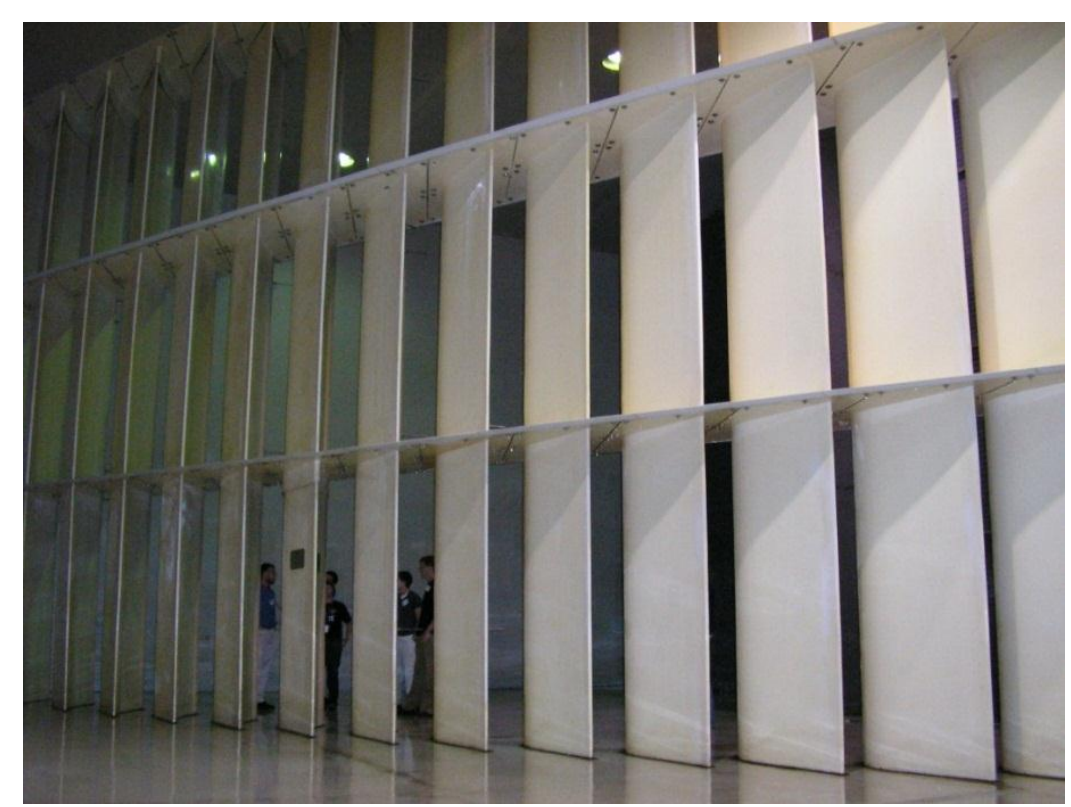

Figure 1.2.5. Photograph looking upstream to the turning vanes on Corner D which guide the flow around the sharp turn. The people standing next to the vanes provide a reference for their size.

A photograph looking downstream to the ten horizontal spray bars can be seen in Fig. 1.2.6. The area incorporating the spray bars and vertical struts before the tunnel's contraction is called the settling chamber. The spray bars are shaped aerodynamically and are vertically spaced two feet from each other while the top and bottom bars are four feet from the tunnel ceiling and floor respectively. At the center of the spray bars is a vertical strut that extends from the floor to the ceiling of the tunnel to provide structural support for the spray bars. In 2009, six additional vertical struts were installed that extend from the top most spray bar to the bottom most spray bar with horizontal spacing of 30 " expect the outer struts which had 60 " of horizontal spacing. These struts were installed to assist in water droplet dispersion by creating additional air flow turbulence near the exit of the water nozzles on the trailing edge of the spray bars. Figure 1.2.7 provides a close-up view of the spray bar and strut intersection with the nozzle ports on the spray bar's trailing edge. The ports are spread 6" from each other, and can contain either Mod-1 
nozzles, Standard nozzles or a sealed plug. The Mod-1 and Standard water nozzles are air assisted and can create a range of different water droplet sizes. The spray bar trailing edge is located 27.5" from the start of the tunnel contraction which can be seen in the background of Fig. 1.2.6.

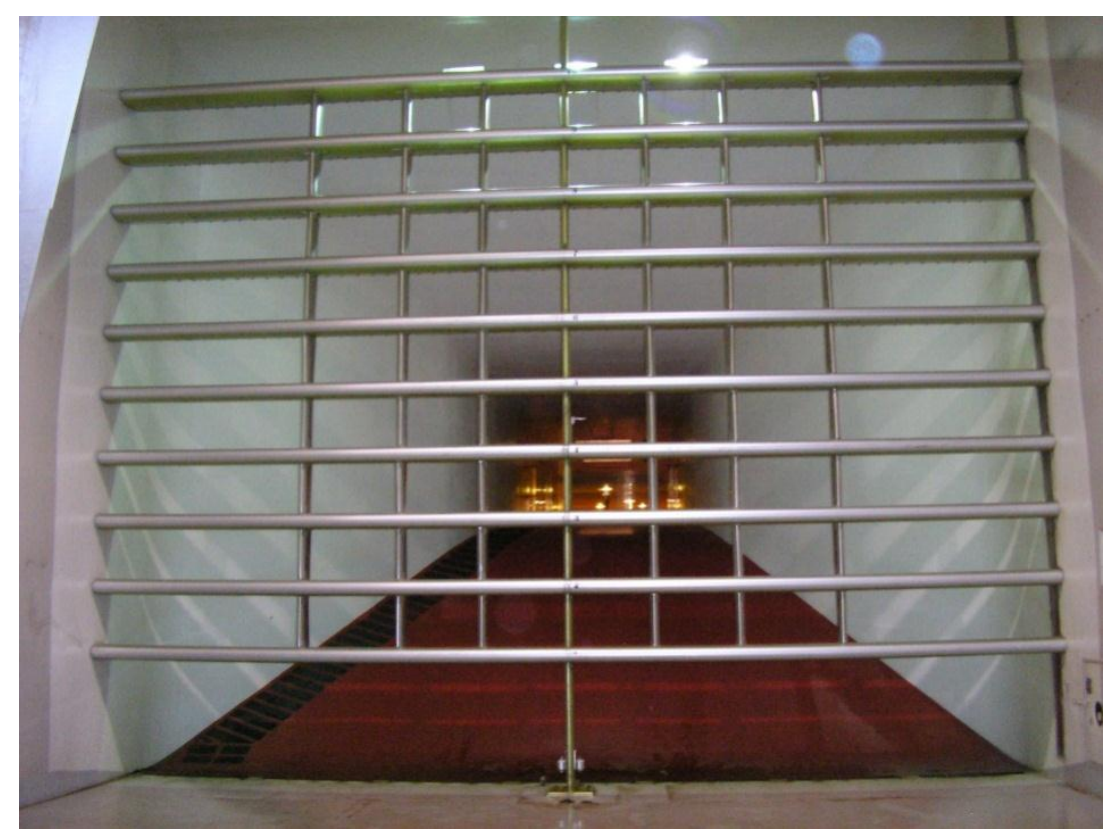

Figure 1.2.6. Photograph looking downstream to the spray bars in the NASA Glenn IRT. The tunnel contraction towards the test section can be seen beyond the spray bars.
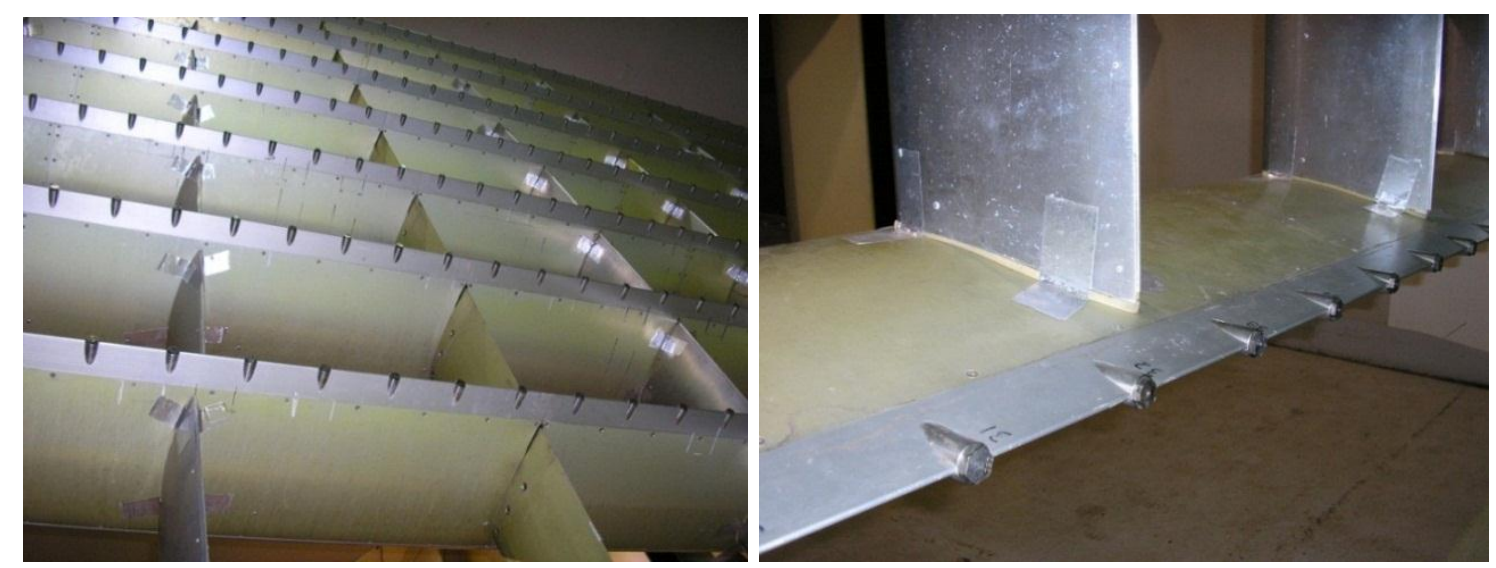

Figure 1.2.7. Close up picture of the spray bar trailing edge with nozzle port locations and vertical struts. 
Figure 1.2.8 is a photograph looking downstream to the tunnel contraction that leads to the test section. The contraction is a 14 to 1 ratio which forms a test section a width of $9 \mathrm{ft}$. and a height of $6 \mathrm{ft}$. Air velocity within the test section can range from 50 to 350 knots and have temperatures as low as -43 Celsius. Windows that provide vision of the test section from the control room are mounted along the entire side of the test section, and can be seen in the background of Fig. 1.2.8. After the test section, the flow continues around corners A and B and recirculates back to the fan. Corners A and B have 1" metal meshes to catch debris to protect the fan from any possible collision damage.

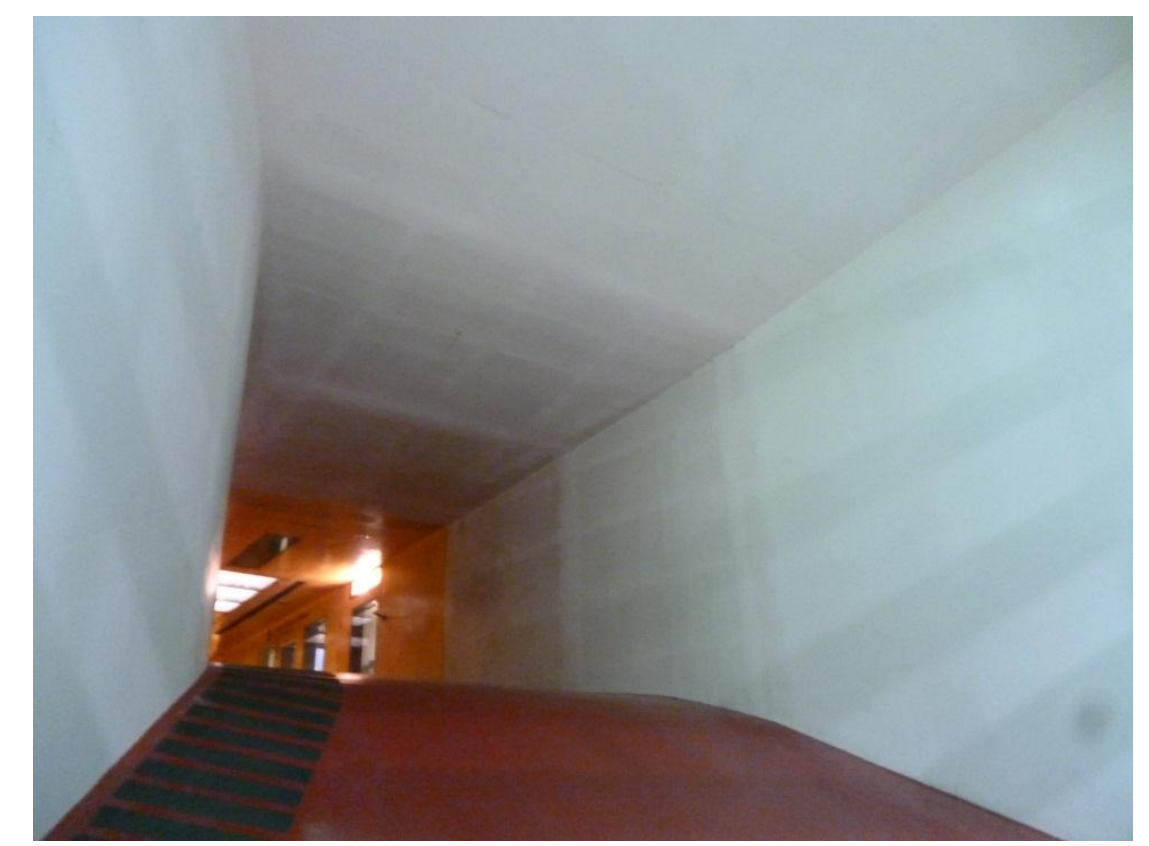

Figure 1.2.8. Photograph of the tunnel contraction that leads into the test section. Windows allowing the control room to view the test section can be seen on the far sidewall.

Major tunnel modifications require the tunnel to be recalibrated for optimal test section conditions in terms of LWC uniformity. To measure this, a $6 \mathrm{ft}$. by $6 \mathrm{ft}$. grid is placed at the center of the test section to accumulate ice. An example of ice buildup on the calibration grid can be seen in Fig. 1.2.9. The ice accumulation should be uniform 
throughout the grid to demonstrate ideal icing conditions at the test section. Figure 1.2.9 shows uneven ice accumulation shown by the varying heights within the ice growth. During the calibration process, the active water nozzles are continually moved to new locations on the spray bars upstream in order to further improve uniformity. With this new nozzle map, the ice accretion on the IRT test section grid is re-evaluated. If the results are unsatisfactory, then the water nozzle locations are altered again. This process continues until an acceptable nozzle map that provides adequate test section conditions is acquired $^{2}$.

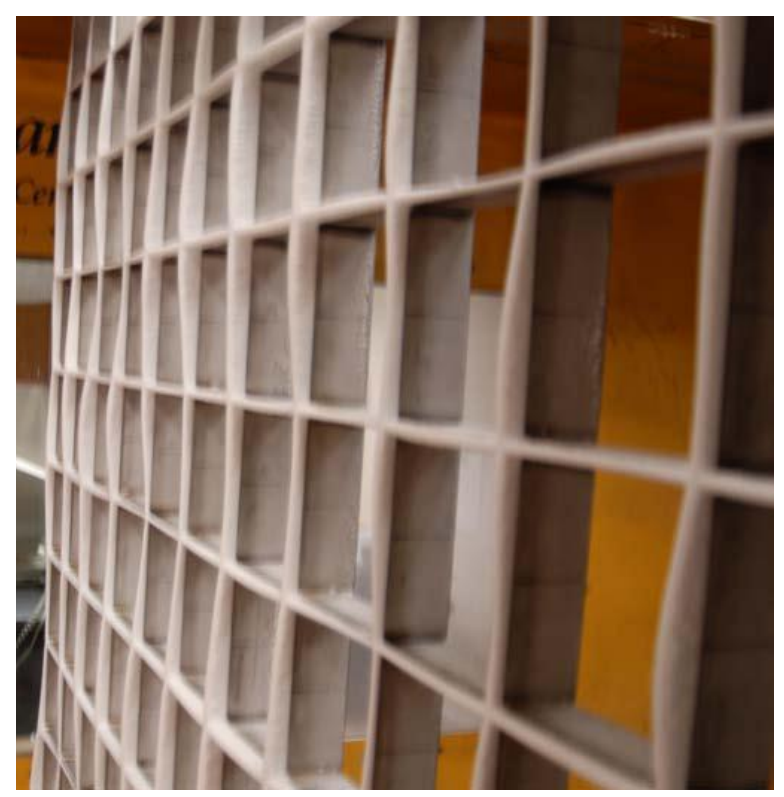

Figure 1.2.9. Ice accumulation on the test section grid used to calibrate the NASA IRT.

\subsection{Previous NASA IRT Simulations}

Because of limitations in computational resources, previous computational simulations of the NASA Glenn Icing Research Tunnel have been limited to modeling certain tunnel sections, i.e., the tunnel contraction and test section. As such, simple computational grids can be utilized by avoiding the complex geometries of the spray bars, 
turning vanes, and other intricate tunnel components. Hancir $e t a l^{3}$ preformed flow field simulations downstream of the spray bars to the test section. By tabulating data from tunnel experiments and individual component simulations and then using a simple linear (empirical) combination of the flow elements (empty tunnel, spray bar element and jet), the inlet boundary conditions were established just downstream of the spray bars. A steady-state compressible flow solution with a $\mathrm{k}-\varepsilon$ turbulence model was used for the air flow and Lagrangian calculations were used for the water droplet trajectories. This simulation matches the NASA IRT configuration before the 1999 modifications which includes a new heat exchanger and increased tunnel width between corners C and D.

Bhargava $e t a l^{4}$ continued the studies performed by Hancir by modifying the simulations to correspond to the NASA Glenn IRT after the 1999 modifications. Bhargava study kept the same methodology that Hancir developed, but developed new inlet boundary conditions that incorporated the flow of the 2000 tunnel configuration. Figure 1.3.1 shows a velocity contour produced from Bhargava which qualitatively looks similar to the Hancir simulations. Figure 1.3.2 illustrates concentration plots of the water droplets at the test section in the NASA IRT from both experiments and simulations. Four water nozzles were active for Fig. 1.3.2 with test section speed of 100 knots and droplets with a mean volumetric diameter (MVD) of 21 microns. These simulations highlighted the importance of the effect air flow turbulence has on droplet transportation and dissipation. Even though the studies showed relatively high degree of agreement with experiments, there is concern in using an empirical linear combination of the turbulence generated by the upstream tunnel components and the use of vertical and horizontal symmetry for the airflow. A fully integrated method by simulating the components 
together for the entire test cross-section would more accurately capture the flow interaction of the various tunnel elements.

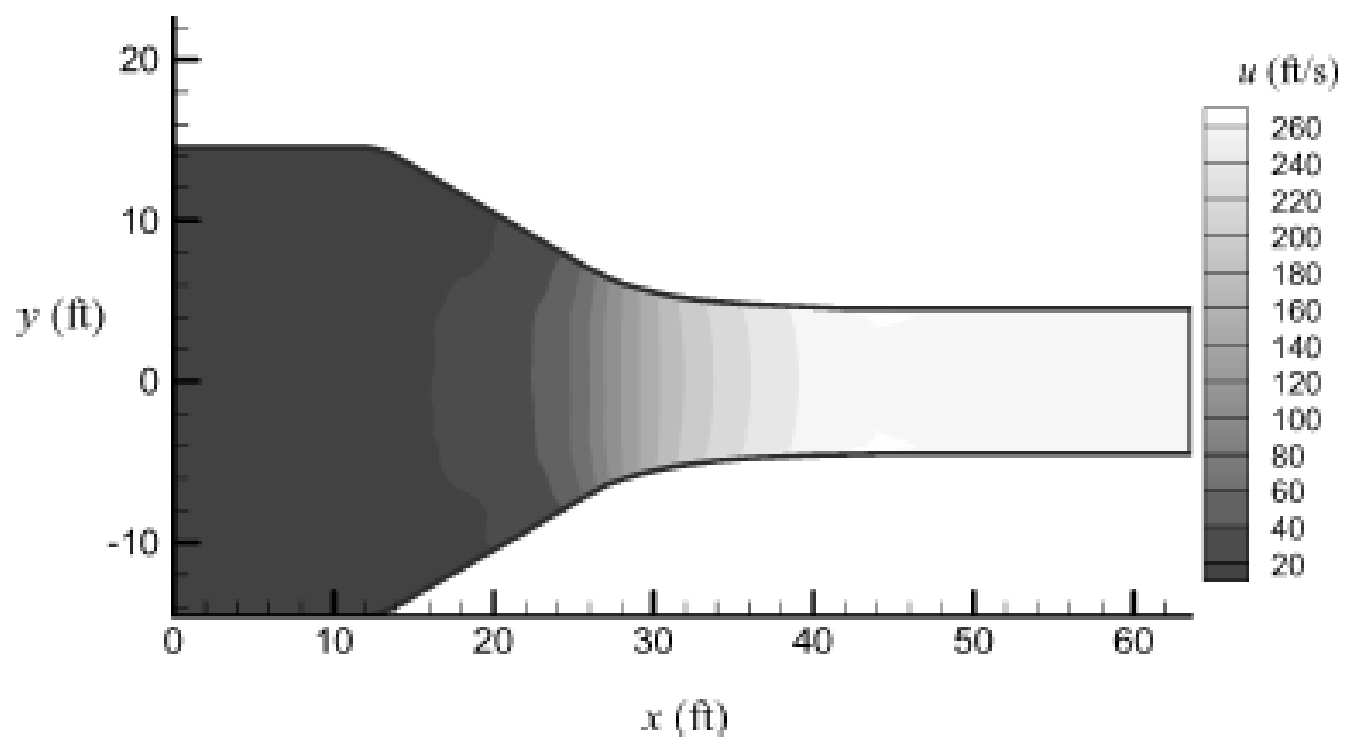

Figure 1.3.1. Velocity contour (ft/s) of the NASA Glenn IRT computed through simulations performed by Bhargava ${ }^{4}$.

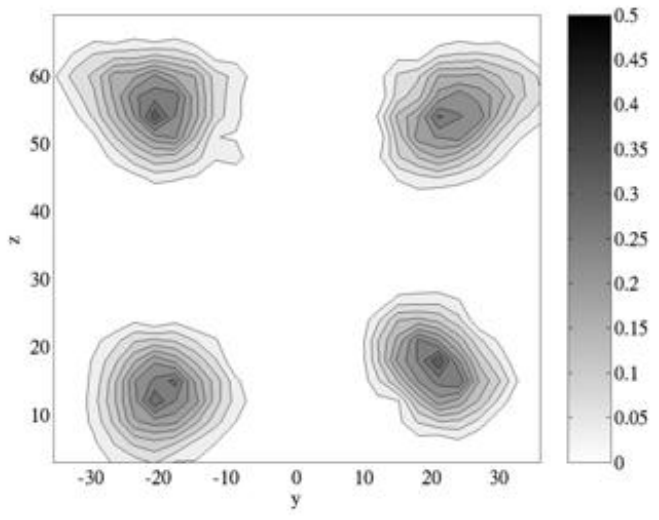

(a)

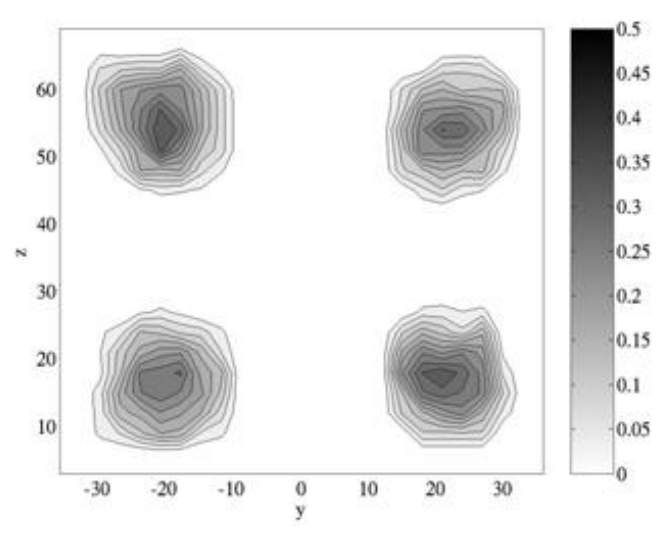

(b)

Figure 1.3.2. Liquid water concentration plots by Bhargava ${ }^{4}$ for four active nozzles tabulated from (a) experiments and (b) simulations. The axes are measured in inches. 


\subsection{Objectives}

The main objective of the current study is to investigate the flow field within the NASA Glenn IRT using CFD to assist in improving Liquid Water Content (LWC) uniformity at the test section. Part I of the study utilizes a Reynolds Average NavierStokes (RANS) method with a Menter SST turbulence model. A RANS model is ideal because it allows for coarser and less uniform computational grids around the complex geometries in the IRT which lowers computational cost. The air flow turbulence generated in the IRT is a focus of the present study and is ideally to be kept to a minimum in the test section. However, turbulence in the settling chamber is helpful for dispersing the water droplets to create a more spatially-uniform LWC, to be consistent with atmospheric conditions. Therefore, it is helpful to understand the influence and dissipation of the flow turbulence to help create optimal conditions for testing to balance the turbulence levels.

For the 2000 and 2009 tunnel configurations, a quarter-tunnel simulation from the spray bars to the test section was used (consistent with previous studies) but with direct integration of all spray bars (avoiding the liner flow field empiricism of the previous studies). In addition, the 2009 configuration simulation incorporated vertical struts directly (these were neglected altogether in previous studies) to represent the tunnel modifications in 2009 and to analyze their impact on the tunnel's turbulence. Finally, air jets were included in the simulation to analyze the increase in turbulence from active nozzles. The test section turbulent kinetic energy was analyzed and compared to 
experiments to validate the RANS model and understand the impact of the different tunnel components.

To model the effect of a new heat exchanger installed in 2012 on the tunnel turbulence, a Corner D simulation was added to the computational domain, thereby extending the domain further upstream. In addition, top-bottom and left-right symmetry was no longer assumed and the full cross-section of the tunnel was simulated (instead of just one quarter). To produce accurate inlet conditions to the corner, a two-dimensional simulation was performed on a section of the heat exchanger which is immediately upstream of Corner D. Using this as the input profile to the three-dimensional computational domain allowed the flow to be computed without symmetry assumptions from just upstream of Corner D all the way into the test section. The mean velocity and turbulence throughout this domain and particularly at the test section was recorded and compared to the quarter-tunnel simulation.

Liquid water droplets were also released in the 2012 tunnel configuration to develop LWC scatter and concentration plots at the test section. Lagrangian calculations with a discrete random walk model (DRW) are utilized to calculate the droplet trajectories. Calibration locations for the water nozzles were used as locations for releasing the water droplets to generate a transfer map. The transfer map provided the location of water droplets in the test section released from specific rows and columns of water nozzles. The transfer map also assisted in calibrating the IRT and was utilized to ascertain the accuracy of the RANS model and the Lagrangian calculations. 


\section{Chapter 2. Air Flow Simulations}

\subsection{Methods}

Continuous flows are governed by the laws of conservation: mass, momentum, and energy. These three conservation equations through a fixed volume in tensor notation are

$$
\begin{aligned}
& \frac{\partial \rho}{\partial t}+\nabla \cdot\left(\rho u_{i}\right)=0 \\
& \frac{\partial\left(\rho u_{i}\right)}{\partial t}+\nabla \cdot\left(\rho u_{i} u_{j}\right)=\rho g-\nabla p+\nabla \tau_{i j} \\
& \frac{\partial(\rho e)}{\partial t}+\nabla \cdot\left(\rho e u_{i}\right)=\nabla \cdot\left(u_{j} \tau_{i j}\right)-\nabla \cdot\left(u_{j} p\right)+\nabla \cdot(\kappa \nabla T)
\end{aligned}
$$

These partial differential equations dictate the behavior of the continuous flow and how its properties vary in space and time. Sometimes all three equations are called the NavierStokes equations but usually only the momentum equation (eq. 2.1.2) is called the Navier-Stokes equation. Depending on the situation, these equations can be simplified (e.g. incompressible flow if the peak Mach numbers is less than 0.3 or steady-state solutions if the flow is laminar). The Mach number is defined as,

$$
M=\frac{u}{a}
$$

where $a$ is the speed of sound. For turbulent flow, analytically solving these equations is impossible regardless of their form because of the complexity and non-linear coupling behavior. Thus, one has to solve the equations numerically which can still prove to be quite difficult and computationally intensive for even the simplest flows at modest Reynolds numbers. Reynolds number is defined as,

$$
\operatorname{Re}=\frac{\rho u D}{\mu}
$$


where $\mathrm{D}$ is a reference length, $\mathrm{u}$ is the freestream velocity, $\rho$ is the density of the flow and $\mu$ is the viscosity of the flow.

Reynolds Averaged Navier-Stokes equations (RANS) assume that the flow variables are comprised of a time-average mean component and a fluctuating component shown as

$$
q=\bar{q}+q^{\prime}
$$

By definition the time average of the fluctuating component must be equal to zero, while the time-average of two fluctuating components is not zero, i.e.

$$
\begin{aligned}
& \overline{q^{\prime}}=0 \\
& \overline{q^{\prime} q^{\prime}} \neq 0
\end{aligned}
$$

If the flow is ergodically stationary, the mean of the product fluctuations will converge with time to a constant value at a given point in the flow. In this case, the time-average of the conservation equations yields a steady-state solution, e.g. the momentum equation becomes,

$$
\begin{gathered}
\frac{\partial\left(\rho \bar{u}_{i}\right)}{\partial t}+\frac{\partial\left(\rho \bar{u}_{i} \bar{u}_{j}\right)}{\partial x_{i}}=-\frac{\partial \bar{p}}{\partial x_{i}}+\frac{\partial}{\partial x_{j}}\left[\tau_{i j}-\rho \overline{u_{i}^{\prime} u_{j}^{\prime}}\right] \\
\tau_{i j}=\mu\left(\frac{\partial \bar{u}_{i}}{\partial x_{j}}+\frac{\partial \bar{u}_{j}}{\partial x_{i}}\right)
\end{gathered}
$$

Solving for the time-averaged tensor of the two fluctuating velocities on the right-hand side of Eq. 2.1.9 requires a turbulence model $^{5}$.

While there are many choices for turbulence models, a two-equation model is ideal for the present study as it allows for specification of both an integral time and length scale of the turbulence, which is needed for the droplet dispersion method, discussed 
later. The Menter Shear Stress Transport ${ }^{6}$ (SST) model is perhaps the most commonly used and robust two equation turbulence model and has the form:

$$
\begin{aligned}
& \frac{\partial(\rho k)}{\partial t}+\frac{\partial\left(\rho k u_{i}\right)}{\partial x_{i}}=\tau_{i j} \frac{\partial u_{i}}{\partial x_{j}}-\beta^{*} \rho \omega k+\frac{\partial}{\partial x_{j}}\left[\left(\mu+\sigma_{k} \mu_{t}\right) \frac{\partial k}{\partial x_{j}}\right] \\
& \frac{\partial(\rho \omega)}{\partial t}+\frac{\partial\left(\rho \omega u_{i}\right)}{\partial x_{i}}=\frac{\gamma}{v_{t}} \tau_{i j} \frac{\partial u_{i}}{\partial x_{j}}-\beta \rho \omega^{2}+\frac{\partial}{\partial x_{j}}\left[\left(\mu+\sigma_{\omega} \mu_{t}\right) \frac{\partial \omega}{\partial x_{j}}\right]+2\left(1-F_{1}\right) \rho \sigma_{\omega 2} \frac{1}{\omega} \frac{\partial k}{\partial x_{j}} \frac{\partial \omega}{\partial x_{j}}
\end{aligned}
$$

where $v_{t}$ is the eddy viscosity and $\gamma, \beta$, and $\sigma$ s are all constants. These constants $(\phi)$ are derived as follows,

$$
\phi=\phi_{1} F_{1}+\phi_{2}\left(1-F_{1}\right)
$$

where $\phi_{1}$ and $\phi_{2}$ are constants based on the k- $\omega$ and k- $\varepsilon$ models respectively and $F_{1}$ is a blending function. This blending function $\left(\mathrm{F}_{1}\right)$ depends on the distance from a no-slip surface and allows the model to convert from a k- $\omega$ model near the surface to a $k-\varepsilon$ model in the far-field. The Menter SST is a robust method that utilizes the strengths of both the $\mathrm{k}-\omega$ and the k- $\varepsilon$ methods to model the flow field.

To validate the accuracy of the $k$ - $\omega$ turbulence model for a simple turbulent wake flow, a computational mesh was constructed to match the physical dimensions of the square duct used in experiments by Synder and Lumley ${ }^{7}$. The mesh contains slip walls and a grid resolution of $200 \times 10 \times 10$ nodes with decreased grid spacing near the entrance of the computational domain to resolve the larger flow gradients at that location. The numerical inflow conditions were specified to be equal to the experimental inflow values while the turbulent kinetic energy $(k)$ and specific dissipation rate $(\omega)$ at 20 grid spaces were specified to be $k=3.0354 \mathrm{ft}^{2} / \mathrm{s}^{2}$ and $\omega=435 \mathrm{~s}^{-1}$, respectively. The value for $\omega$ was obtained by converting $\varepsilon$ using the relation 


$$
\omega=\frac{\varepsilon}{c_{\mu} k}
$$

where $c_{\mu}$ is an empirical constant of 0.09. As shown in Fig. 2.1.1, both the turbulent kinetic energy and specific dissipation rate compare favorably to the experimental measurements.

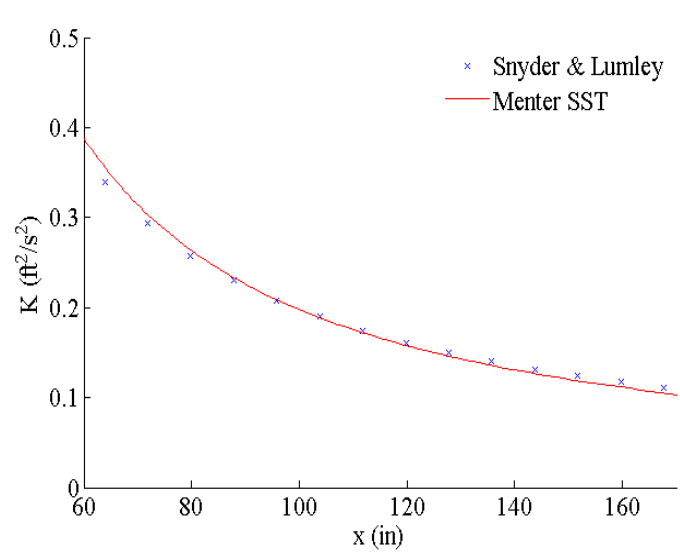

(a)

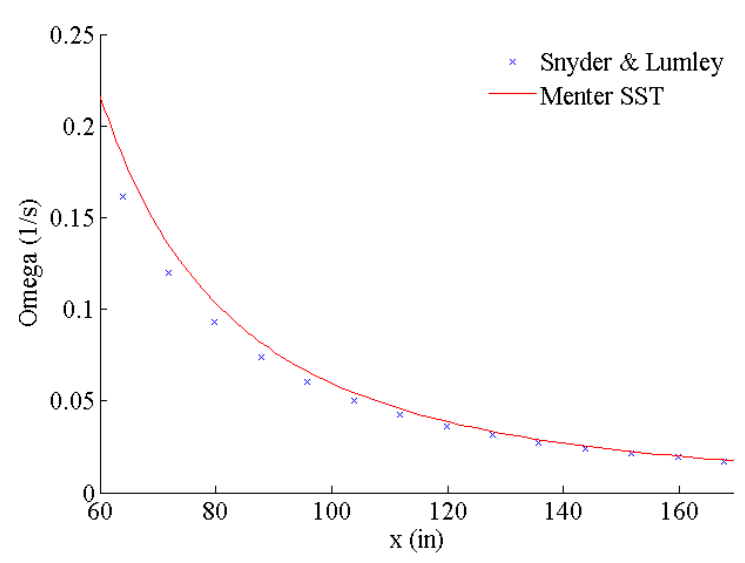

(b)

Figure 2.1.1. Validation of the k- $\omega$ turbulence model with experimental values measured by Snyder and Lumley ${ }^{7}$ showing (a) turbulence and (b) specific dissipation rate.

A semi-implicit method for pressure-linked equations (SIMPLE) pressurevelocity coupling scheme was utilized to close the conservation of mass and momentum equations. A SIMPLE technique requires an initial estimate of the pressure $\left(p^{*}\right)^{n}$ and momentum $\left(\rho u_{i}^{*}\right)^{n}$ to solve for a new momentum value $\left(\rho u_{i}^{*}\right)^{n+1}$ at every cell within a domain. A new pressure value $\left(p^{*}\right)^{n+1}$ is calculated at every cell and generates an entire new set of values for the next iteration. This process is repeated until a measure of convergence is satisfied ${ }^{8}$. A second-order upwind scheme was utilized to spatially discretize the momentum and the Menter SST equations. 
The RANS method described above was employed by the FLUENTv12.1 software packaged provided by the ANSYS Corporation. The process to invoke the described methods in FLUENT via the user interface can be found in Appendix A. Grid generation and modifications were done within the Gridgen software that imported to the FLUENT software for calculations. Data analysis and contour generation for visual interpretations were completed in the TECPLOT software. Additional post-processing of the data for comparisons to experimental data were performed in MATLAB and Microsoft EXCEL

To integrate the jet flows into the tunnel simulation, it was first necessary to simulate the aerodynamics of a single jet. The geometry of the Mod-1 (Fig. 2.1.2) is used to compute the near-field jet flow starting at the nozzle exit. Since the flow speed is chocked and at supersonic when leaving the nozzle exit, a RANS density-based model was used to account for the compressibility effects while the turbulence was still modeled with the Menter-SST model. To validate this approach, a computational study of a single jet flow using the Mod-1 nozzle exhausting into quiescent conditions was conducted and compared with experimental results obtained by Bulzan et al. ${ }^{9}$ The experiment consisted of a Mod-1 air-assist nozzle positioned within a $5.9 \mathrm{ft}$ x $5.9 \mathrm{ft}$ x $7.8 \mathrm{ft}$. enclosure with the spray directed vertically downward with air and water mass flow rates of $2.25 \mathrm{~g} / \mathrm{s}$ and $0.49 \mathrm{~g} / \mathrm{s}$ respectively at a pressure of $20 \mathrm{psig}$. Jet flow and particle flow characteristics at various axial distances were then obtained using Laser Doppler Anemometry (LDA) and Phase/Doppler instrumentation, respectively. 


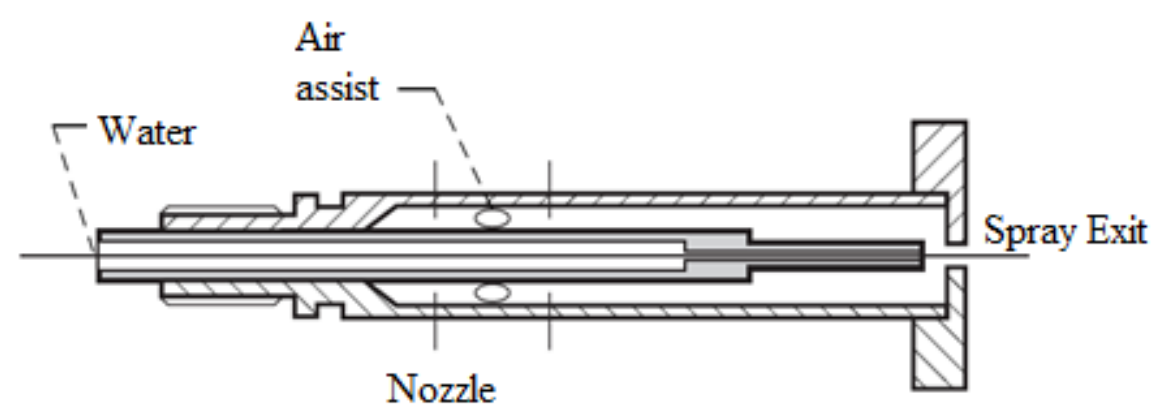

Figure 2.1.2. Schematic of Mod-1 nozzle where flow moves left to right. ${ }^{13}$

As shown in Fig. 2.1.3 a two-dimensional axisymmetric mesh consisting of approximately 8500 node points was constructed for the single jet domain. The computational domain measures 35"x 10" and the nozzle was placed at the bottom left corner with inflow conditions matching the Bulzan ${ }^{9}$ experiments, i.e. choked with zero nozzle exit turbulence and dissipation. To satisfy choked conditions with the prescribed mass flow rate and assuming a uniform and unidirectional exit velocity from the nozzle, the effective radius of the nozzle in the computational domain was set to be 0.0448 " to account for vena contraction effects as discussed by Lee et al. ${ }^{10}$. Figure 2.1.4 shows contours of the results for the jet flow simulation. It can be observed in Fig. 2.1.4 that the flow exits from the nozzle and becomes supersonic leading to the formation of shock diamond patterns before spreading and slowing down to subsonic speeds. This jet flow is accompanied by a shear layer as shown in Fig. 2.1.4 due to the interaction of the core flow with the surrounding air. Since the single jet computation employs a twodimensional axisymmetric mesh, the computational time required for a converged solution is quite reasonable. 


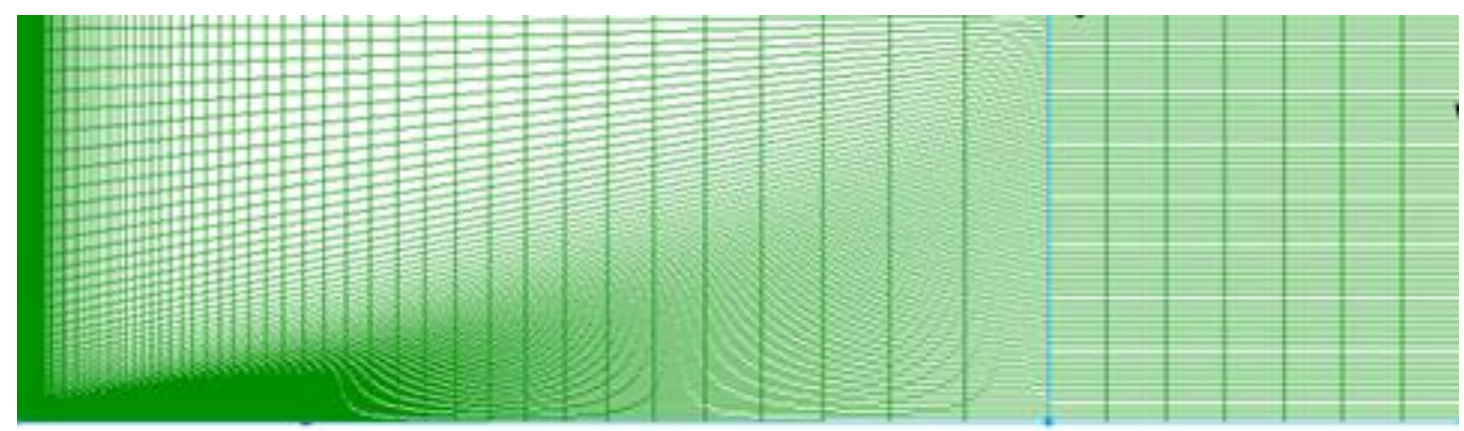

Figure 2.1.3. Computational mesh and domain for the simulation of an air jet from the Mod-1 nozzle.

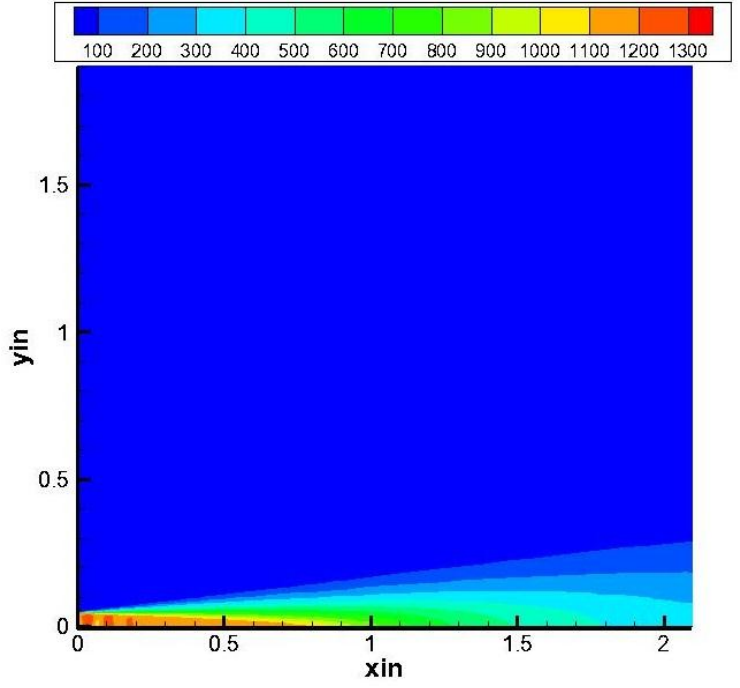

(a)

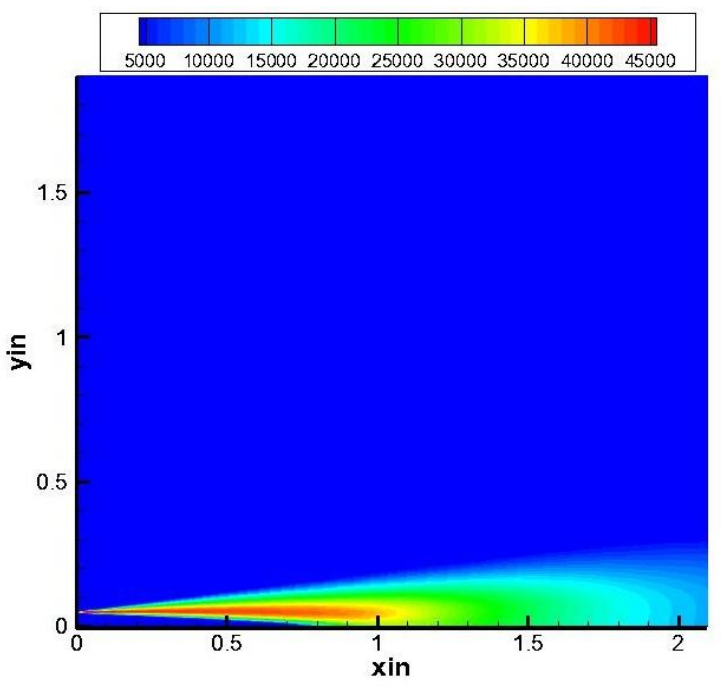

(b)

Figure 2.1.4. Contours of the axisymmetric simulation of the air jet from the mod-1 nozzle showing (a) velocity in $\mathrm{ft} / \mathrm{s}$ and (b) turbulence in $\mathrm{ft}^{2} / \mathrm{s}^{2}$.

In contrast to the single jet simulation, incorporating an array of a hundred or so of these jet flows with a three-dimensional grid that resolved each of their near-fields into the entire three-dimensional IRT domain would be highly impractical in terms of both excessive computational time and grid points. Both problems stem because of the large number of grid points and large variation in length scales required to capture the small scale flow structures in all the jet near-fields (where the supersonic portion of each jet 
flow are limited to a few millimeters) while distributing these over the entire of the tunnel (where the cross-section height and width are on the order of 30 feet). To allow this flow to be feasible computationally, a jet profile was obtained at 4" downstream from a single jet computational domain and this profile was applied as velocity inlet boundary condition within the IRT domain. This allows the IRT computational grid to avoid submillimeter grid resolution and also allows its solution to employ a pressure based solver due to the lack of compressibility effects, when more than 4 " downstream of the jet (where the centerline Mach number has been reduced to 0.15). Data obtained at 4" downstream from the nozzle simulation was used as the velocity profile to compute the far-field.

To determine the accuracy of the profile used as the initial condition profile for the overall IRT domain, a far-field single jet computational domain was constructed with a two-dimensional axisymmetric mesh that used the 4" profile as an inflow condition. The 4" profile encompasses 1.2 " radially from the jet centerline which encompasses the entire jet flow at 4" with the velocity negligible at the outer radius. Restricting the profile to only 1.2 " allows for flow characteristics generated upstream to have an influence on the jet flow. The profile is incorporated into the domain using a combination of a pressure outlet and a velocity inlet boundary condition. The pressure outlet is facing upstream and set to match the current flow field while the velocity inlet faces downstream set to the obtained jet profile and initiates the jet flow for the far-field domain. Figure 2.1.5 shows the far-field axisymmetric velocity and turbulence contours where it can be seen that this 4" profile boundary condition produced physically consistent flow field evolutions, without introducing non-physical oscillations upstream or downstream. 


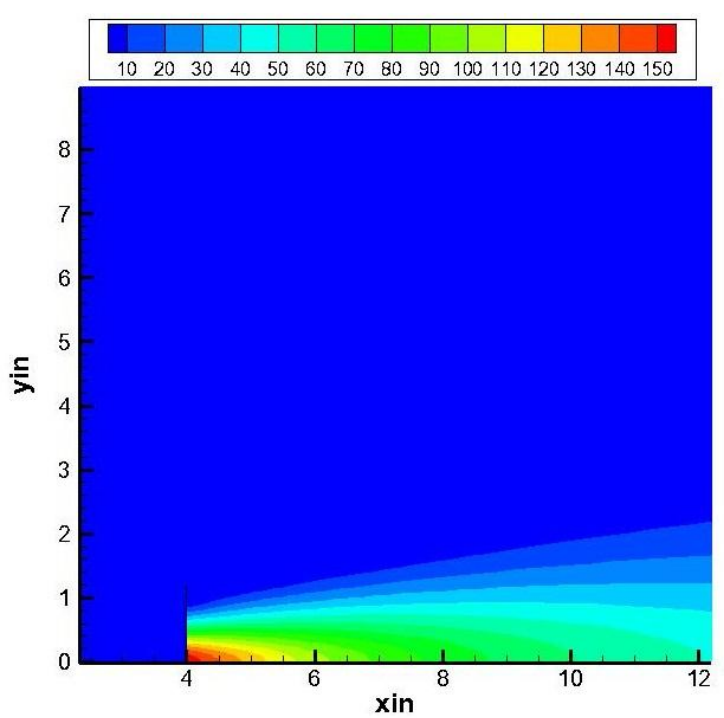

(a)

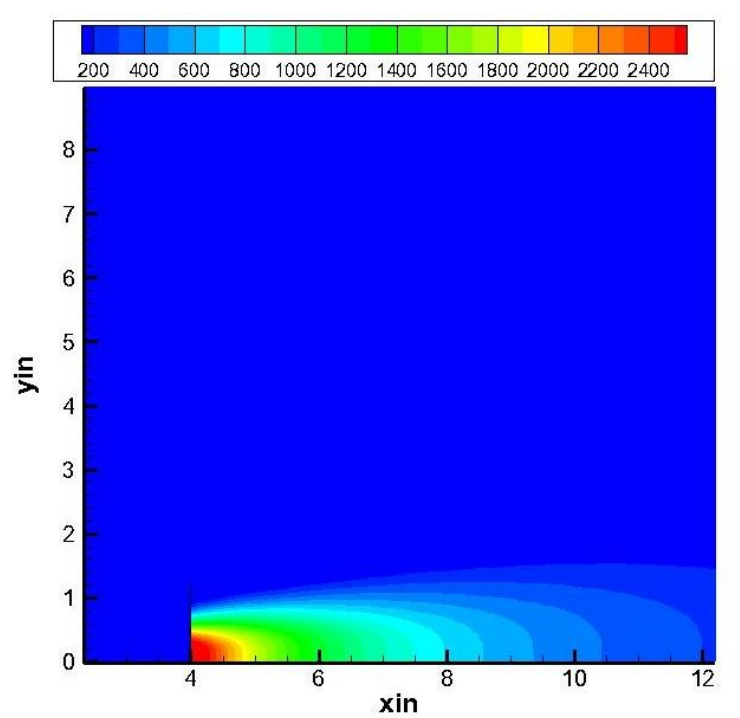

(b)

Figure 2.1.5. Contours of the axisymmetric simulation of the jet flow initiated at 4" downstream of nozzle exit showing (a) velocity in $\mathrm{ft} / \mathrm{s}$ and (b) turbulence in $\mathrm{ft}^{2} / \mathrm{s}^{2}$.

The far-field predictions and those of the near-field simulations were compared with experimental results. Figures 2.1.6 and 2.1.7 compare the experiments from Bulzan with the simulations from the nozzle and the 4" velocity profile. The simulations and experiments differ by $6 \%$ and $10 \%$ at the centerline $20 \mathrm{~cm}$ downstream (Fig. 2.1.7) for the velocity and turbulence respectively. The simulations compare even more favorably as the radius increases which illustrate the model is reproducing reasonable flow physics. Since the differences between the simulations from the nozzle and the simulation using the 4" profile are negligible (compared to differences with experimental results), implementing 4" profile within the IRT simulations is also expected to be reasonable. 


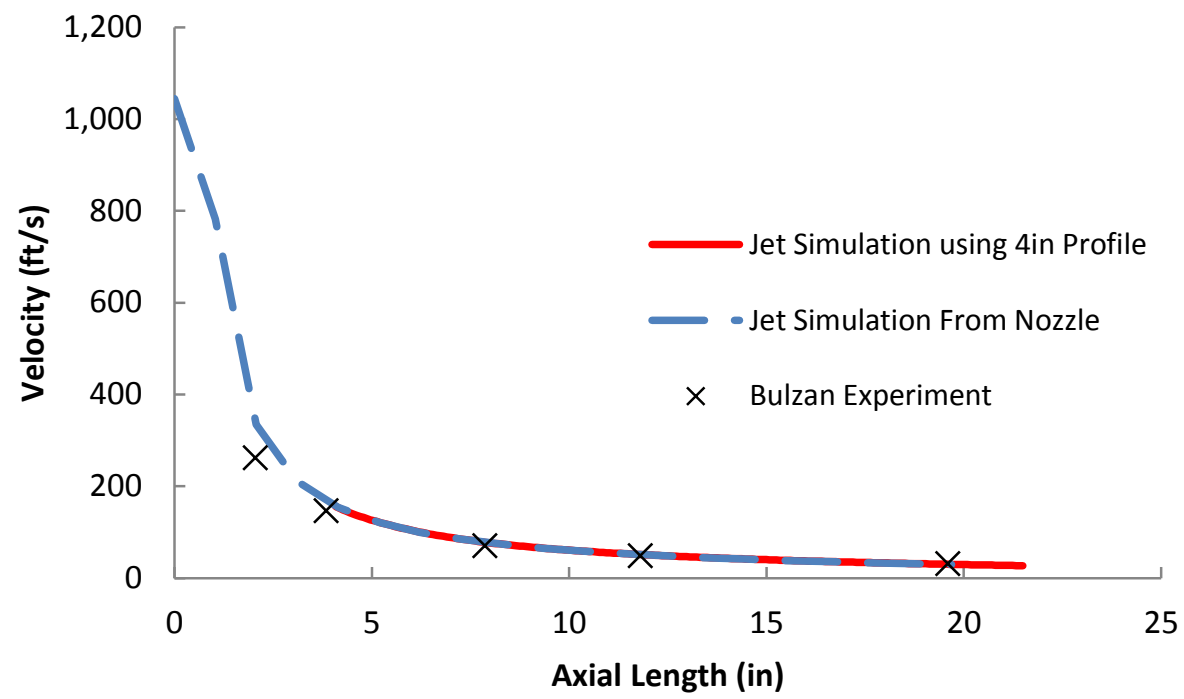

Figure 2.1.6. Comparison of axial jet flow velocity between simulations from nozzle and 4" profile with experimental data from Bulzan". 


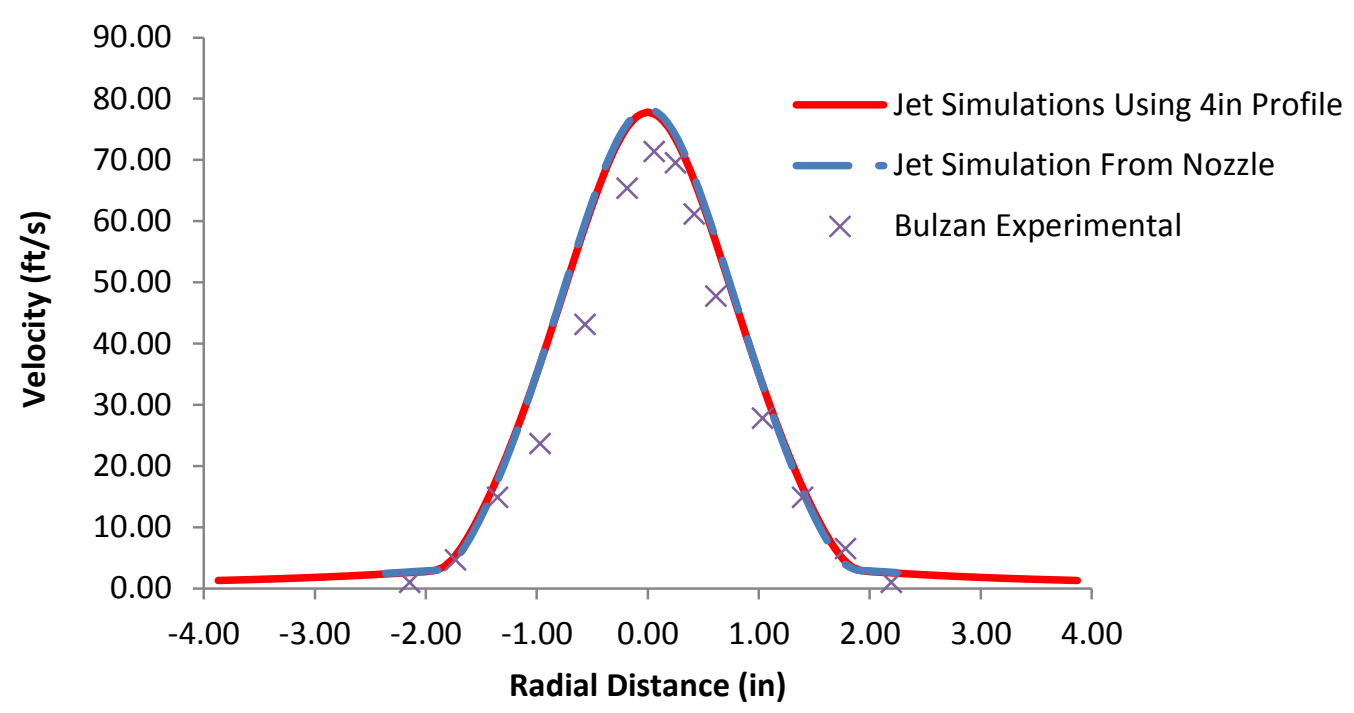

(a)

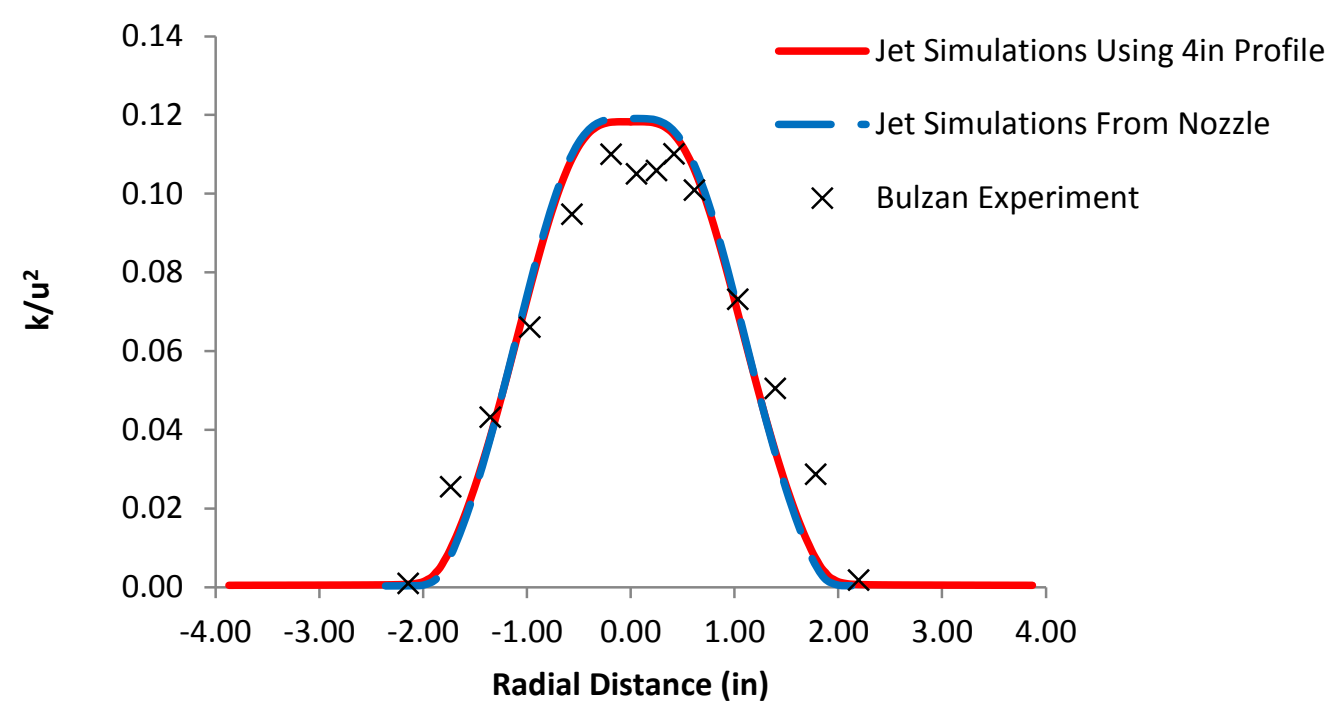

(b)

Figure 2.1.7. Comparison of radial profiles 7.9 " $(20 \mathrm{~cm})$ downstream from nozzle for predictions with experimental data from Bulzan ${ }^{9}$ (a) velocity profile and (b) normalized turbulence. 


\subsection{Quarter-Tunnel for 2000 and 2009 IRT Configurations}

To allow for comparison with experiment and previous studies, simulations were performed on a domain that included the spray bars and extended to the test section, for both the year 2000 and 2009 tunnel configuration. The year 2000 tunnel geometery does not include the additional vertical struts on the spray bars added in 2009. A side view of the grid used in the spray bar only simulation for the 2000 IRT configuration can be seen in Fig 2.2.1. This domain extends 80 " before the spray bars to the center of the test section. Due to the assumed symmetry within the IRT, only the upper left of the tunnel when looking upstream, is simulated. These simulations used an uniform inflow with a velocity of $18.1 \mathrm{ft} / \mathrm{s}$ and an outlet pressure of $14.1 \mathrm{psi}$ to maintain a velocity around 175 $\mathrm{mph}$ in the test section to compare with experiments by Gonsalez ${ }^{1}$. Based on data processed previously by Bhargava ${ }^{4}$, the inflow turbulent kinetic energy, $k$ was specified to be $0.53 \mathrm{ft}^{2} / \mathrm{s}^{2}$ and the specific dissipation rate, $\omega$ given by $4.08 \mathrm{~s}^{-1}$. The walls and the spray bars are treated as no-slip surfaces while the tunnel center-line was modeled with symmetry boundary conditions. The grid is a structured mesh with C-grids around the spray bars with a dimensionless wall distance $\left(y^{+}\right)$of 1 for the first grid spacing close to the spray bars and walls. The grid was coarsened before the spray bars and during the tunnel contraction and has a total of 774,000 cells.

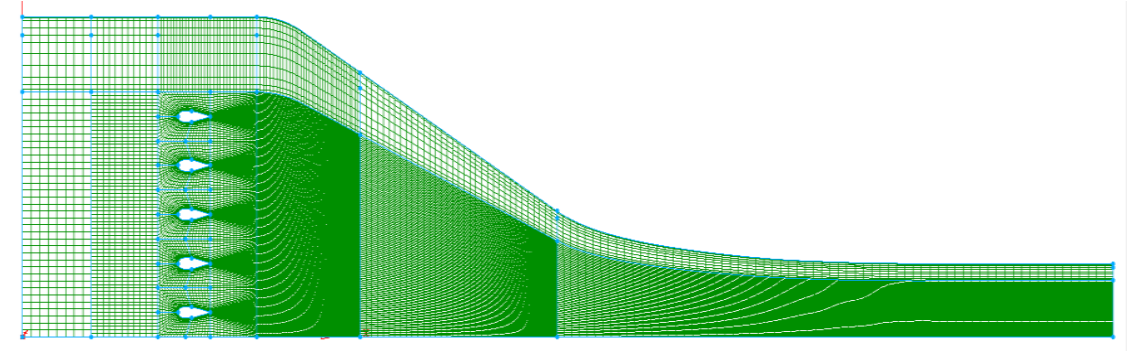

Figure 2.2.1. Computation domain of the quarter-tunnel simulation of the 2000 IRT configuration with spray bars only. 
Figure 2.2.2 shows contours of the mean velocity and turbulence at the spray bar region for the quarter-tunnel simulations of the 2000 tunnel configuration. The contours are taken at a vertical slice located at the center of the tunnel. The main flow interactions in the tunnel are the wake of the spray bars and the contraction of the tunnel leading to the test section. The upper spray bars create a wake that curves downwards as compared to the relatively straight wakes seen behind the spray bars near the centerline. This wake on the upper spray bars creates higher turbulent kinetic energy immediately below the trailing edge. Due to the symmetry condition at the center of the tunnel, the bottom spray bars in the tunnel will generate similar turbulence effects. In previous studies by Bhargava and Hancir, this three-dimensional effect was not observed since the spray bar wakes were assumed to be identical and taken from a single 2-D solution (i.e. the spray bars were previously not gridded within the IRT domain).

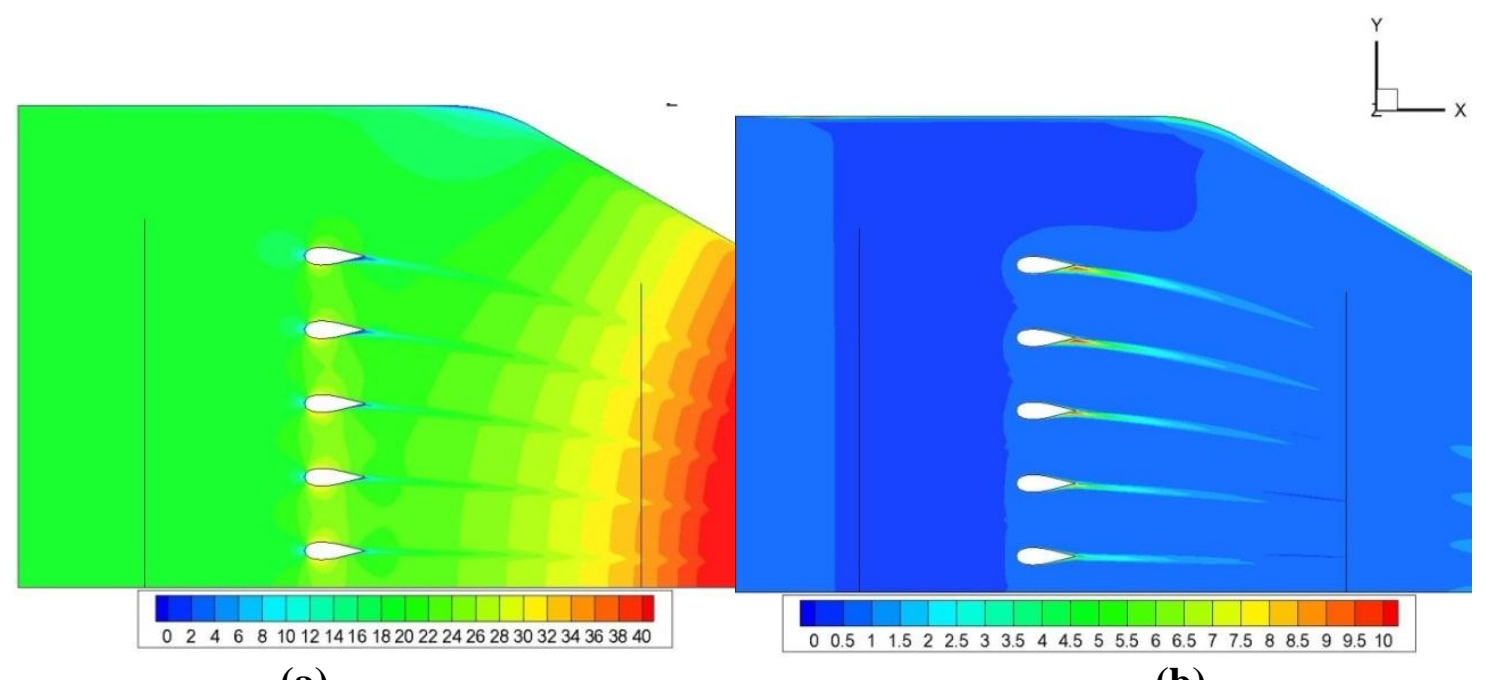

(a)

(b)

Figure 2.2.2. (a) Contour plot of mean velocity in $\mathrm{ft} / \mathrm{s}$ and (b) contour plot of the turbulent kinetic energy in $\mathrm{ft}^{2} / \mathrm{s}^{2}$ for 2000 configuration. These slices were taken at the center of IRT (right side of quarter-tunnel simulation).

Test section turbulent kinetic energy contours can be seen in Fig. 2.2.3. All of the contours of the air flow are generated looking upstream (i.e. the left side represents the 
outer wall). The highest turbulent kinetic energy is located at the center of the tunnel and the outer walls (top and left side of the contour). The turbulence at the center is generated from the wakes of the spray bars located upstream while the high turbulence at the walls can be attributed to the wall boundary layers. Table 2.2.1 compares the velocity fluctuations of the test section with experiments from Gonsalez ${ }^{1}$ using the same data point locations. The RANS predicted velocity fluctuations described within Table 2.2.1 are based on a isotropic turbulence model and therefor assume that the turbulence intensity is the same in all directions, i.e..

$$
u_{r m s}=u_{x, r m s}=u_{y, r m s}=u_{z, r m s}
$$

where $u_{r m s}$ is the root-mean-square of the velocity fluctuations and $u_{x, r m s}, u_{y, r m s}$, and $u_{z, r m s}$ are in the three different directions. Table 2.2.1 shows the measured velocity fluctuations for both the streamwise and vertical directions during experiments and the isotropic RANS based on the following relation ${ }^{11}$.

$$
u_{x, r m s}=u_{y, r m s}=\sqrt{\frac{2}{3} k}
$$

The present predictions are bounded by the two velocity fluctuations that were measured from the experiments. The predictions show a $13 \%$ difference from the average velocity fluctuations $(1.28 \mathrm{ft} / \mathrm{s})$. Considering the empiricism of the RANS turbulence model and the assumption of isotropy, the $13 \%$ percent error is reasonable for the RANS model and demonstrates reasonable results at the test section. 


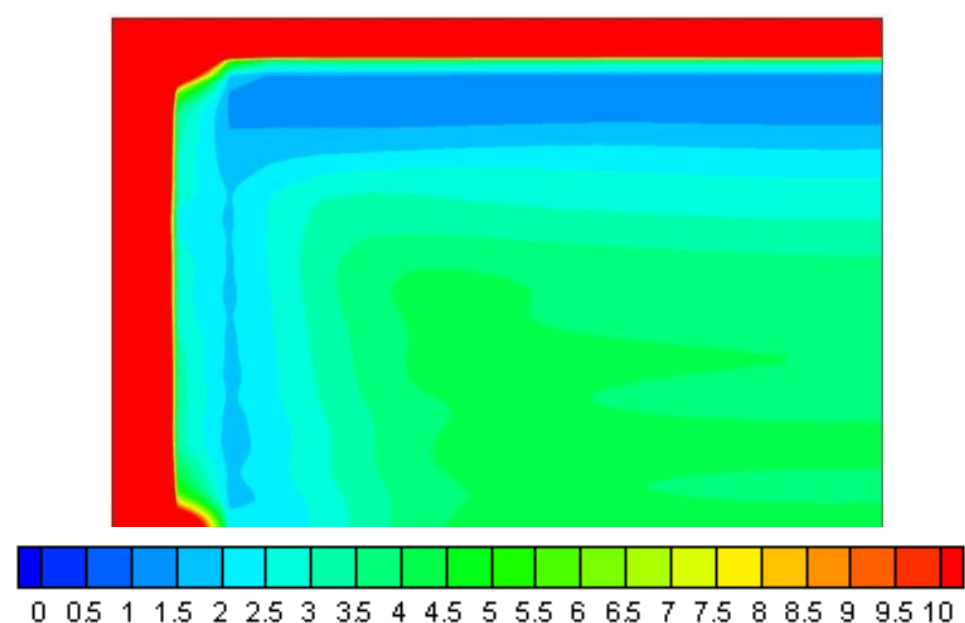

Figure 2.2.3. Contour of turbulent kinetic energy in $\mathrm{ft}^{2} / \mathrm{s}^{2}$ at test section for quarter-tunnel simulation of the 2000 IRT configuration without active jets. The air flow contours were generated looking upstream (i.e. the left side represents the outer wall and the right side represents the center of the tunnel).

\begin{tabular}{|c|c|c|c|}
\hline Source & Jets & $\begin{array}{c}\mathbf{u}_{\mathbf{x}, \mathbf{r m s}} \\
(\mathbf{f t} / \mathbf{s})\end{array}$ & $\begin{array}{c}\mathbf{u}_{\mathbf{y}, \mathbf{r m s}} \\
(\mathbf{f t} / \mathbf{s})\end{array}$ \\
\hline Present Predictions & No Jets & 1.48 & 1.48 \\
\hline Gonsalez $^{1}$ & No Jets & 1.03 & 1.54 \\
\hline
\end{tabular}

Table 2.2.1. Test Section velocity fluctuation comparison between Gonsalez ${ }^{1}$ experiments and the present simulation for 2000 tunnel configuration (i.e. without struts).

Experiments done in early 2009 led to implementing additional vertical struts to assist in dispersing the liquid water droplets. The simulation with spray bars and vertical struts uses the same inlet and outlet boundary conditions as the 2000 (spray bars only case). The grid is also similar to the quarter-tunnel simulation of the 2000 tunnel configuration, but with an increased number of cells in the spanwise direction. This is needed to resolve the vertical strut boundary layers which increased the total number of cells to four million. Contours of the mean velocity and kinetic energy on a spray bar and strut intersection can be observed in Fig. 2.2.4. The slice of the contours is taken in the middle of the strut located 60 " from the spanwise center of the tunnel. There is higher 
turbulent kinetic energy directly behind the spray bar and strut intersection as compared to the spray bar only case due to the two wakes interacting. For the upper spray bars, the flow below an intersection has more turbulence then above an intersection because of the wake converging downwards towards the center of the tunnel. The spray bar near the center, lowest on Fig 2.2.4, has a more even separation at the intersection. The increased turbulence at the right end in Fig 2.2.4 is from the wake of an outer strut converging towards the center of the tunnel. Figure 2.2.5 displays the progression of the turbulence along the tunnel from the spray bars to the test section. The turbulence is shown to be increasing and converging towards the center as the flow accelerates moves towards the test section, but remains relatively constant while flowing through the straight test section.

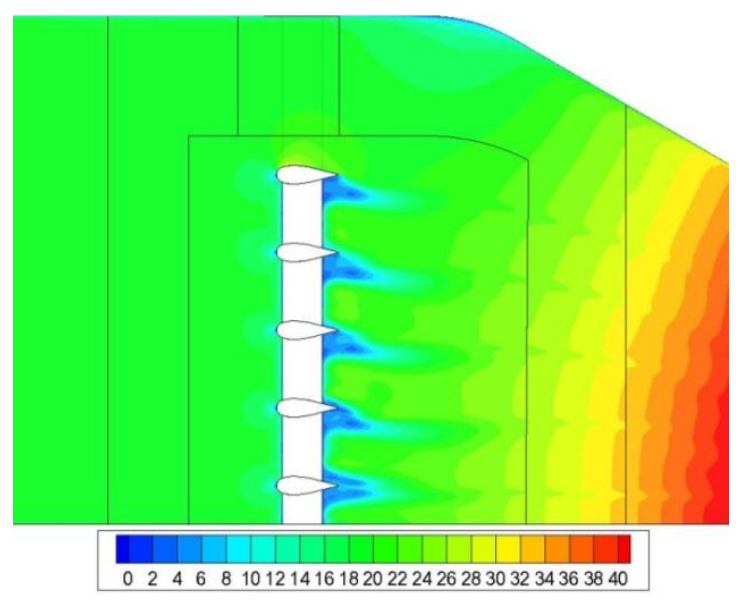

(a)

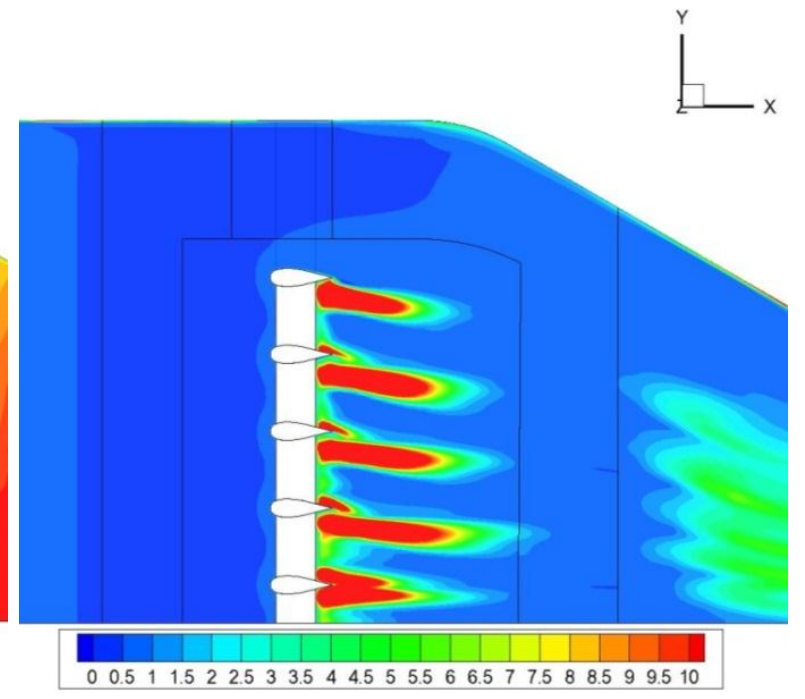

(b)

Figure 2.2.4. (a) Contour plot of the mean velocity in $\mathrm{ft} / \mathrm{s}$ and (b) contour plot of turbulence in $\mathrm{ft}^{2} / \mathrm{s}^{2}$ in the middle of the strut 60 " from center of the tunnel for 2009 tunnel configuration without jets. 


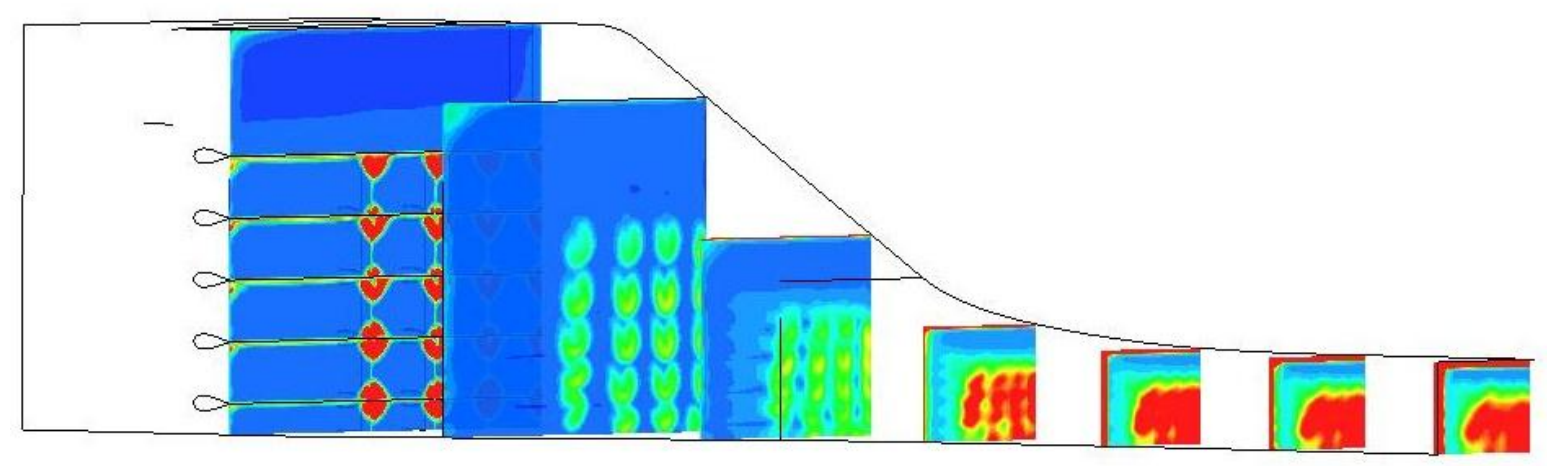

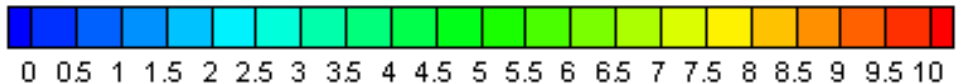

Figure 2.2.5. Contour slices of turbulence in $\mathrm{ft}^{2} / \mathrm{s}^{2}$ starting at the spray bars to the test section for quarter-tunnel simulation of the 2009 IRT configuration without jets.

Figure 2.2.6 shows the contour of the turbulent kinetic energy at the test section for the 2009 tunnel configuration without jets. This contour indicates that the walls and the center of the test section contain the highest amount of turbulent kinetic energy which is similar to the 2000 tunnel configuration. However, the turbulent kinetic energy is much higher at the test section center for this configuration when compared to the 2000 configuration in Fig. 2.2.3. This is caused by the additional turbulent kinetic energy from the wakes produced by the struts and the high turbulence generated by the intersections between the spray bars and struts. 


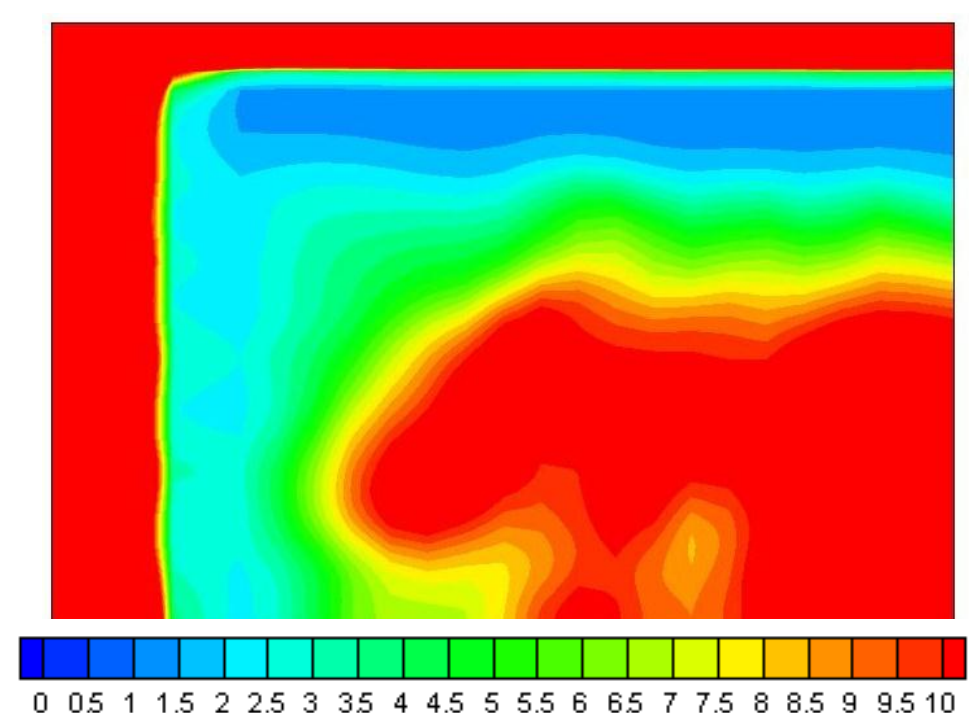

Figure 2.2.6. Contour of turbulent kinetic energy in $\mathrm{ft}^{2} / \mathrm{s}^{2}$ at test section for quarter-tunnel simulation of the 2009 IRT configuration without jets.

The 2009 tunnel configuration was used to implement the jet velocity profiles into a larger tunnel simulation using the procedure described in section 2.1. The velocity profiles were implemented 4" downstream of the spray bars according to 2009 jet configuration provide by NASA Glenn found in Fig. 2.2.7. The velocity profiles were modified for a 30 psig jet instead of the previous 20 psig to correspond with experiments provided by Van Zante ${ }^{12}$. Figure 2.2 .8 shows a velocity contour streamwise slice 4" downstream of the spray bars where the velocity profiles for the jets are positioned. The high velocity points are where the jets are active for this tunnel configuration. The low velocity locations are due to the spray bar and vertical strut wake interaction. Figure 2.2.9 displays the contour slices of the turbulence along the length of the tunnel. Comparing Fig. 2.2.9 to Fig. 2.2.5 shows that the jets cause higher distribution of the high turbulent kinetic energy instead of having only high turbulence behind a strut and spray bar interaction. However, once the test section is reached the difference between 
having jets implemented and without jets implemented is not as high as one might initially expect. This can be seen in Fig. 2.2.10.

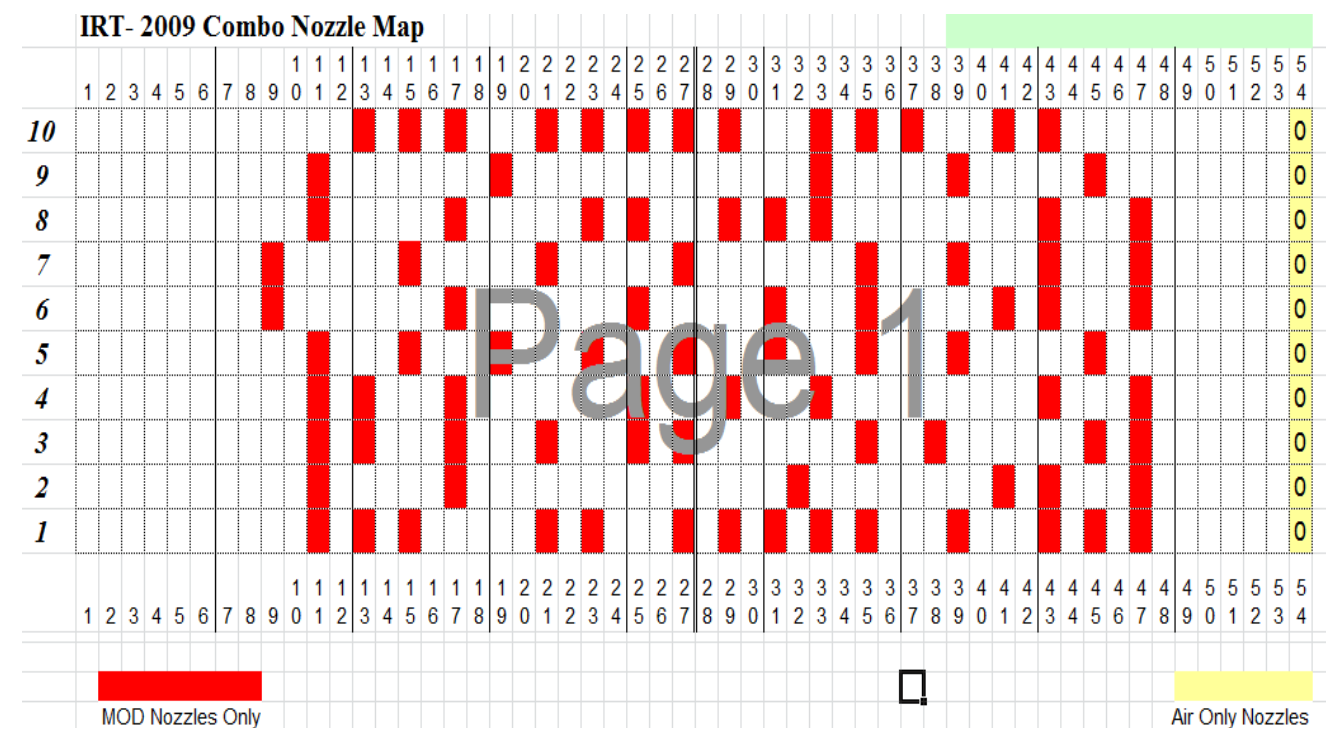

Figure 2.2.7. Map of the nozzle locations for 2009 tunnel configuration. The map was created looking upstream, i.e. the right side represents the inner wall of the IRT ${ }^{12}$.

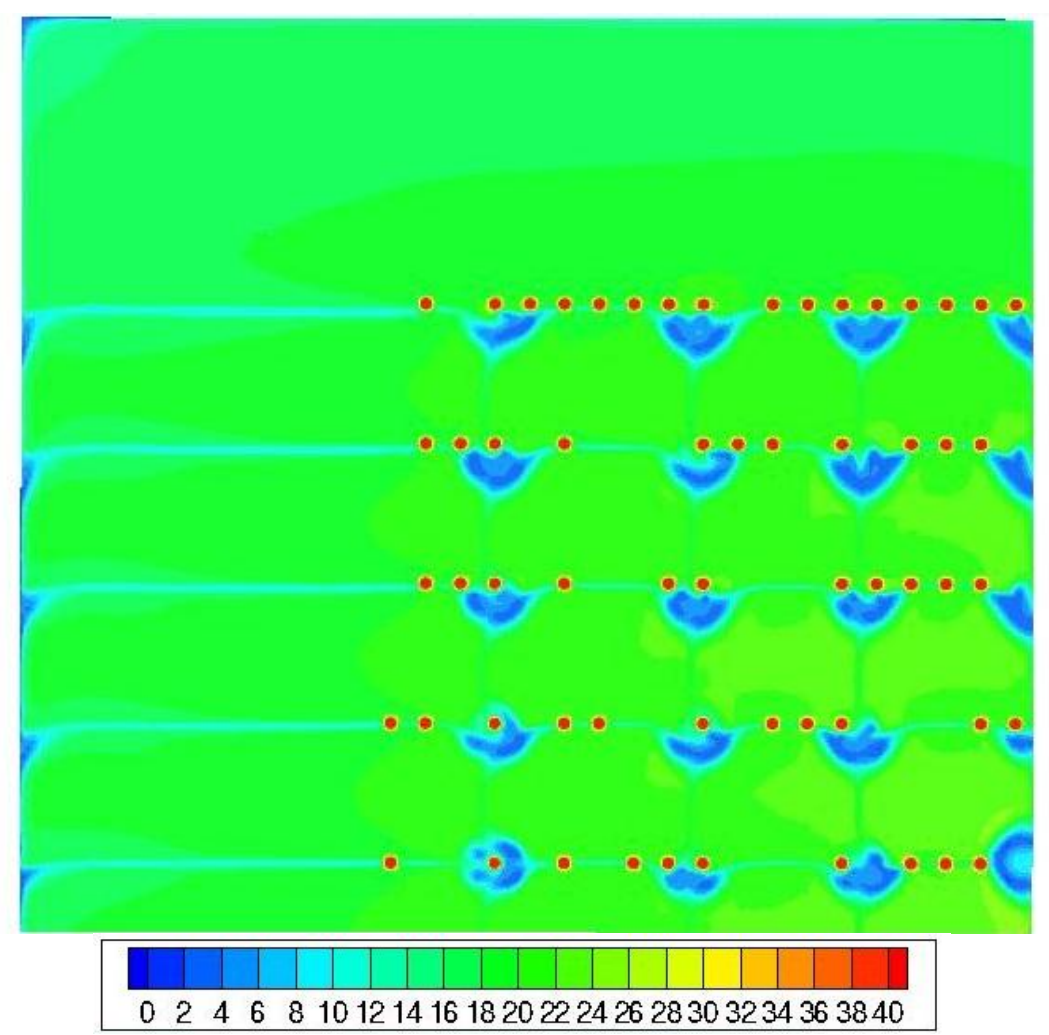

Figure 2.2.8. Velocity contour in ft/s of a vertical slice 4" downstream of spray bars in the quarter-tunnel simulation of the 2009 IRT configuration with jets. The red circles of high velocity are the jet locations. 

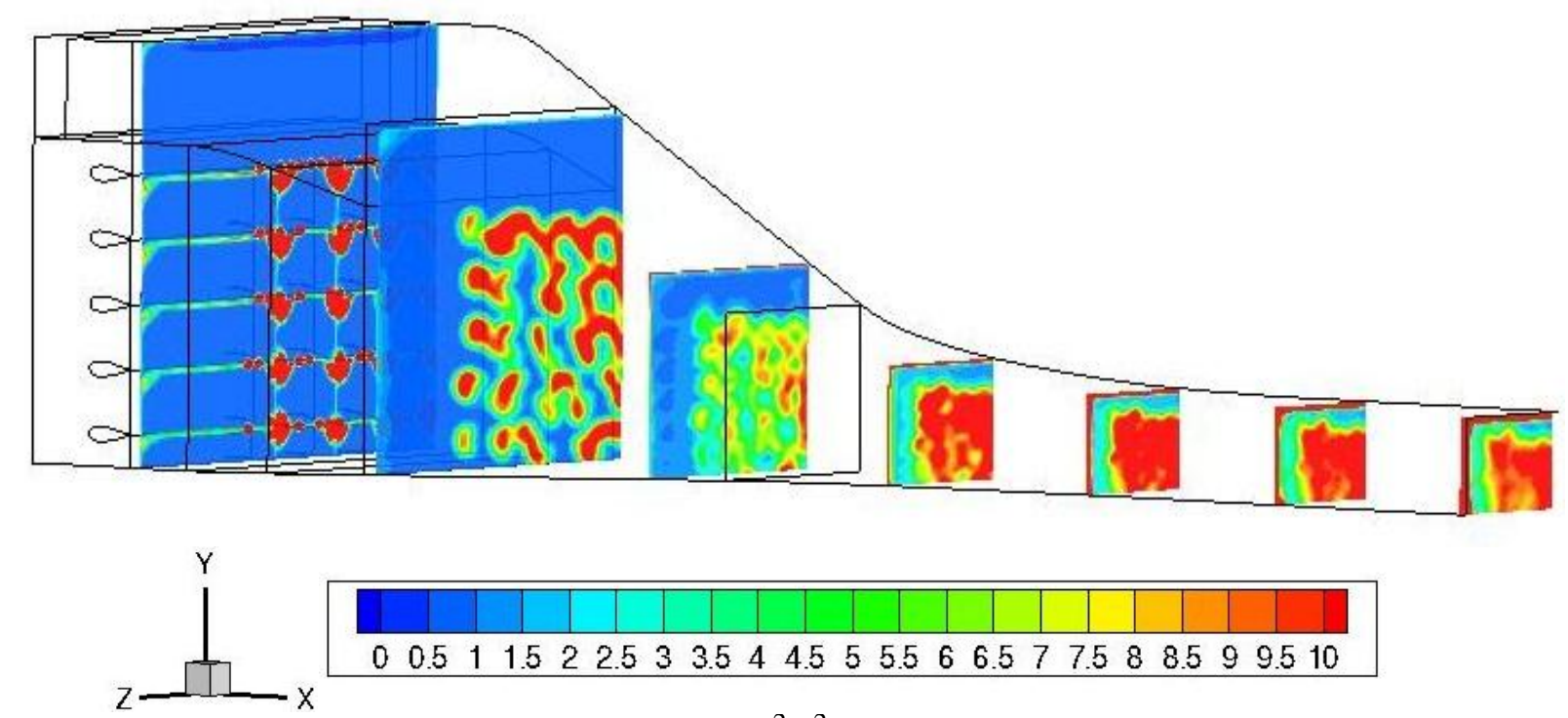

Figure 2.2.9. Contour slices of turbulence in $\mathrm{ft}^{2} / \mathrm{s}^{2}$ starting at spray bars to the test section for 2009 configuration with jets.

Figure 2.2.10 displays the turbulent kinetic energy at the test section for the simulation with the jets implemented and is similar to the results without jets. The high turbulence with jets implemented is more spread across the test section while the simulation without jets is more concentrated at the center, but the intensity between the two cases is approximately the same. Table 2.2.2 compares the simulations of the 2009 tunnel configuration with experiments provided by Van Zante ${ }^{12}$. These experiments used hot wire probes to obtain the $\mathrm{u}_{\mathrm{x}, \mathrm{rms}}$ but did not obtain any $\mathrm{u}_{\mathrm{y}, \mathrm{rms}}$ or $\mathrm{u}_{\mathrm{z}, \mathrm{rms}}$ values and the $\mathrm{u}_{\mathrm{x}, \mathrm{rms}}$ for the present predictions assume isotropic turbulence. The current simulations under predict the intensity of the velocity fluctuations by $30 \%$ without jets and $18 \%$ with jets. The under predictions may be due to highly unsteady flow associated with vertical struts and spray bar interaction (as discussed in Part II, RANS substantially underpredicts the kinetic energy associated with flow separation on a spray bar). These quarter- 
tunnel simulations were modeled after the 2000 and 2009 tunnel configurations which will be altered in 2012 with a new heat exchanger.
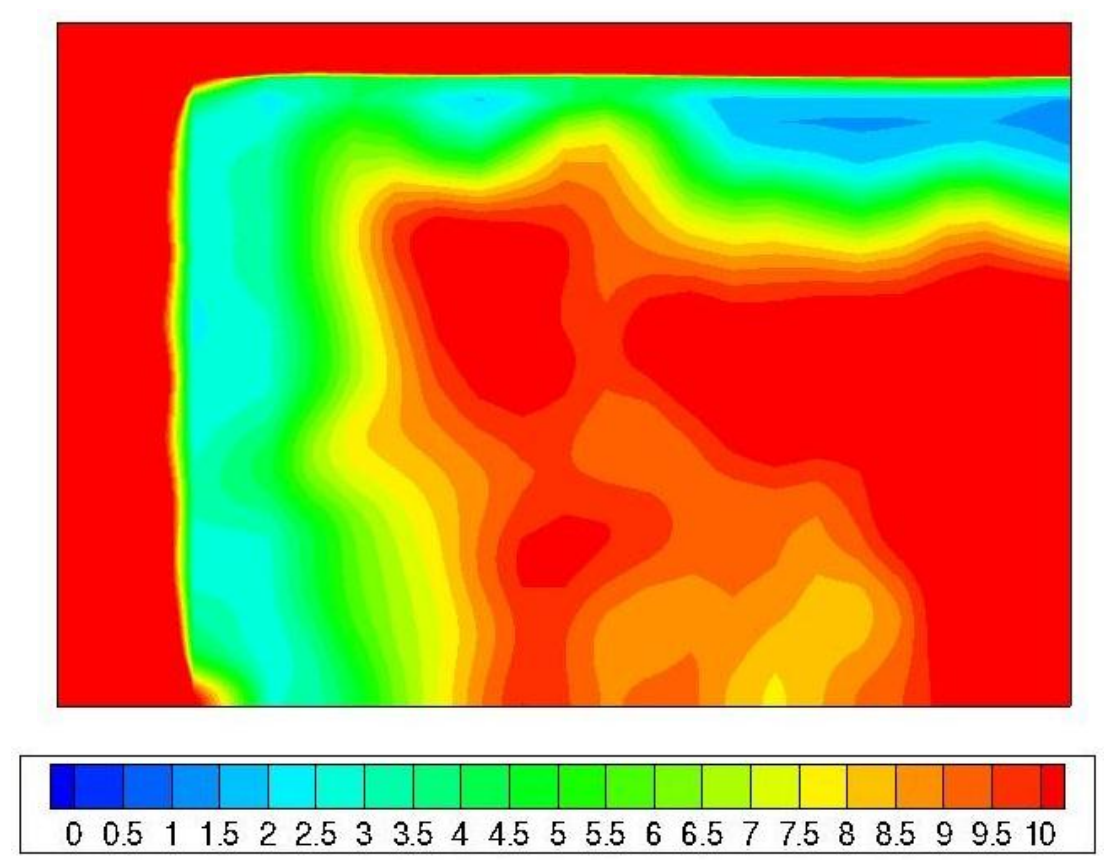

Figure 2.2.10. Contour of turbulent kinetic energy in $\mathrm{ft}^{2} / \mathrm{s}^{2}$ at test section for the quartertunnel simulation of the 2009 tunnel configuration with jets.

\begin{tabular}{|c|c|c|}
\hline Source & jets & $\mathbf{u}_{\mathbf{x}, \mathbf{r m s}}(\mathbf{f t} / \mathbf{s})$ \\
\hline Present & no & 2.27 \\
\hline Van Zante & no & 3.26 \\
\hline Present & yes & 2.48 \\
\hline Van Zante & yes & 3.03 \\
\hline
\end{tabular}

Table 2.2.2. Test section velocity fluctuation for Van Zante experiments ${ }^{12}$ and present simulations for 2009 tunnel configuration with struts.

\subsection{2-D Simulations of the 2012 Heat Exchanger}

A new heat exchanger was installed in 2012 to improve the temperature control and uniformity within the NASA Glenn IRT. The new heat exchanger is designed with rows of pipes stacked at a 30 degree angle from the tunnel floor seen in Fig 2.3.1. This shows an outline for the two-dimensional grid used to model one section of the 2012 heat 
exchanger. The outline is a side-view perspective of the inner tunnel wall (i.e. the air flow moves from left to right through the heat exchanger). The locations of the pipes are shown by the circles and are arranged in rows at the 30 degree angle. A single section of the heat exchanger is outlined in Fig. 2.3.1; the entire heat exchanger consists of six of these sections stacked vertically from tunnel floor to ceiling. In comparison, the previous flat panel heat exchanger used for simulations by Bhargava ${ }^{4}$ and the previous quartertunnel simulations had a more uniform positioning for its pipes without the angled rows. The vertical variations in the 2012 heat exchanger geometry are expected to cause additional flow disturbances not seen generated by the previous heat exchanger. To better understand the flow field in the 2012 tunnel configuration, investigations were performed to characterize the 2012 heat exchanger exit flow.

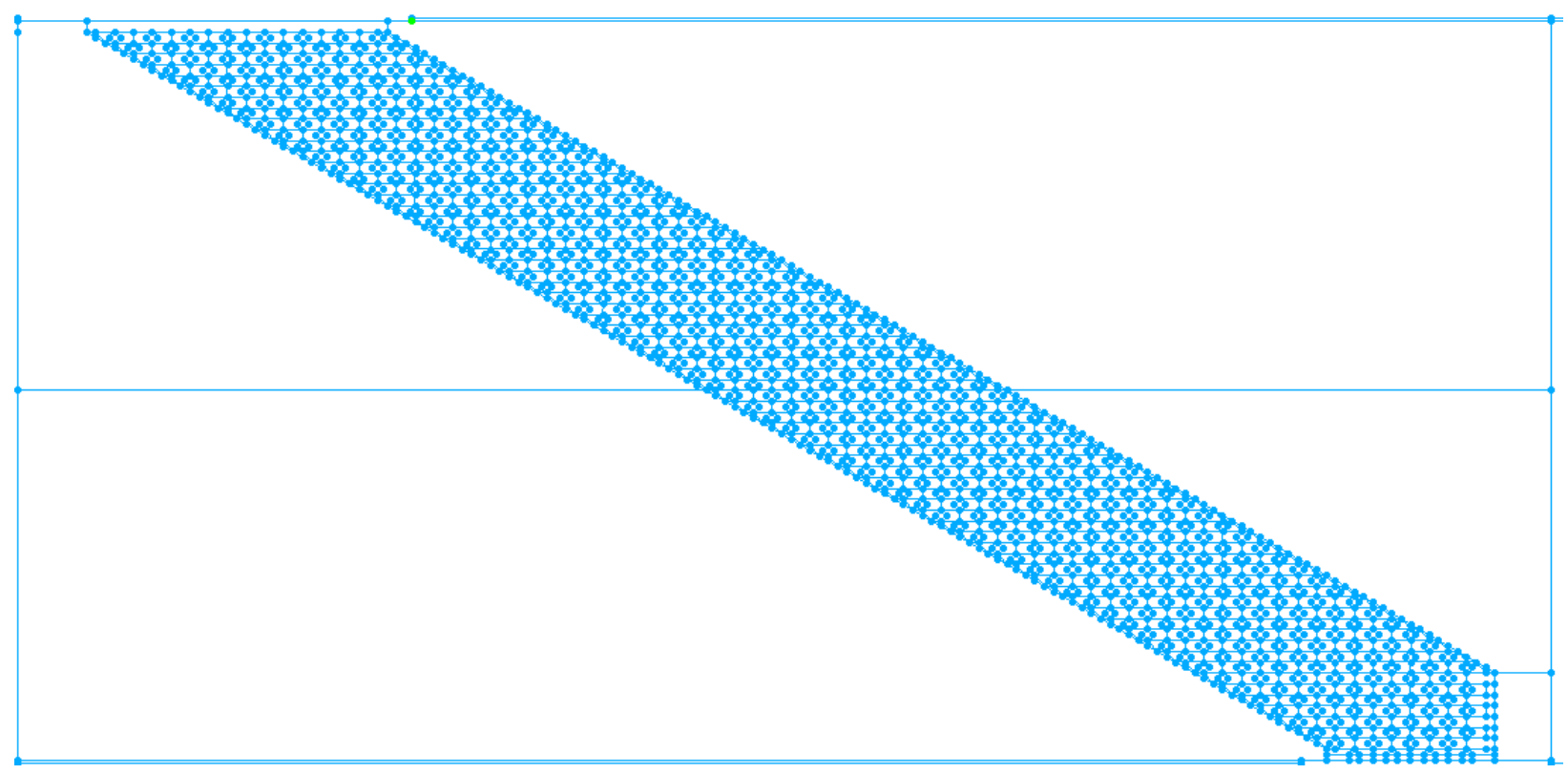

Figure 2.3.1: Outline for the computational grid used to model one section of the 2012 heat exchanger. 
Each section of the 2012 heat exchanger consists of 512 different pipes laid horizontally. Figure 2.3.2 displays a zoomed-in view of the two-dimensional computational grid around the pipe and highlights the detailed grid spacing required to properly capture the complex flow around each pipe. Simulating the entire heat exchanger with the six different sections arranged vertically would be computationally expensive, even for a two-dimensional simulation. Simulating a portion of the 2012 heat exchanger allows for the complex flow around the pipes to be examined without the need to simulate the entire heat exchanger.

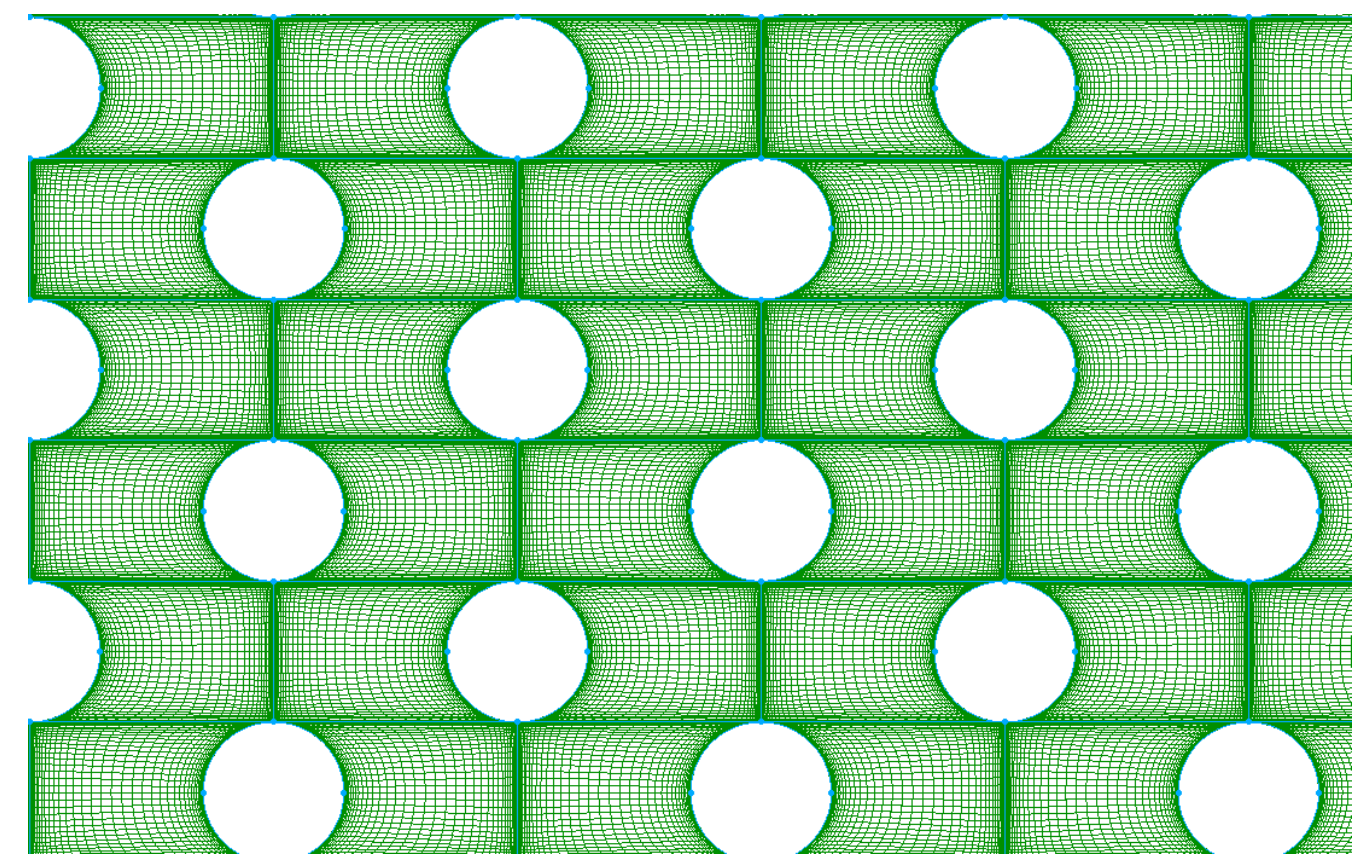

Figure 2.3.2. Zoomed in view of the computational grid around the pipes within the 2012 heat exchanger.

The initial simulation included only a single row of pipes with periodic boundary conditions on the top and bottom boundaries. This single-row computational solution forced the flow to non-physically leave either the top or bottom boundary and be reintroduced into the simulation downstream. A second row of pipes was added to avoid forcing all of the flow through a periodic boundary condition. However, the two-row 
simulation showed that the air flow favored moving between the rows that did not incorporate the periodic boundaries. Realistically, the air flow would enter the heat exchanger uniformly between the different rows and would not favor one row over another. Additional rows were included to increase the simulated flow area that would avoid the periodic boundary conditions on the top and bottom boundaries. When multiple rows of pipes (4-8 rows) were simulated a trend becomes evident that the top rows induce a higher flow velocity than the bottom rows. As additional rows were included the velocity gradient between the top and bottom rows increased. To investigate this flow phenomenon and as well as analyze the interaction between the air flow and the splitter plate that separates the different heat exchanger sections, an entire section of the heat exchanger was eventually modeled in a two-dimensional simulation as seen in Fig. 2.3.1.

The outline in Fig. 2.3.1 extends from the upper supporting bracket to the lower supporting bracket of one heat exchanger section creating a 106 " by 51.5 " domain. The supporting brackets are plates that support the pipes and mount to the splitter plates that separate the different sections of the heat exchanger. To incorporate the entire splitter plate, the domain was extended 12" upstream (to the left) and 12" downstream (to the right). Another 36" were added downstream to simulate the wake that will form behind the trailing edge of the splitter plate and to ensure that any outlet boundary conditions would not affect the flow within the heat exchanger. The entire domain consists of 2.2 million grid points with $\mathrm{y}^{+}$values of 1 near all the no-slip walls. The pipes, supporting bracket, and splitter plate were modeled as no-slip walls while the top and bottom boundaries past the splitter plate are modeled as a slip wall. The slip boundary allows for realistic conditions when incorporating the outlet into the full IRT RANS simulations at 
the tunnel ceiling and floor. An inlet with a $10.75 \mathrm{ft} / \mathrm{s}$ streamwise velocity and minimal turbulent kinetic energy and dissipation was set for the left inlet boundary with the assumption that the air flow would be nearly uniform after Corner C. The right boundary was set as a pressure outlet with atmospheric conditions.

Figure 2.3.3 displays the velocity contour of the air flow through one section of the heat exchanger. The black arrow marks the location of the splitter plate trailing edge. The bottom portion of the section shows very low velocity due to the wake from the bottom rows of pipes. The negative values of streamwise velocity suggest strong recirculation in this region. As the flow moves past the end of the splitter plate it starts to increase in velocity. Having such low velocity in the lower region of the section forces the upper region to have much higher velocity to sustain mass continuity in the flow. These high velocity gradients greatly increase the turbulent kinetic energy especially downstream of a splitter plate where two heat exchanger sections connect. At the splitter plate, the bottom heat exchanger section will have the high velocity seen at the top of Fig. 2.3.3 while the upper section will have the low velocity in the wake seen at the bottom of Fig 2.3.3.

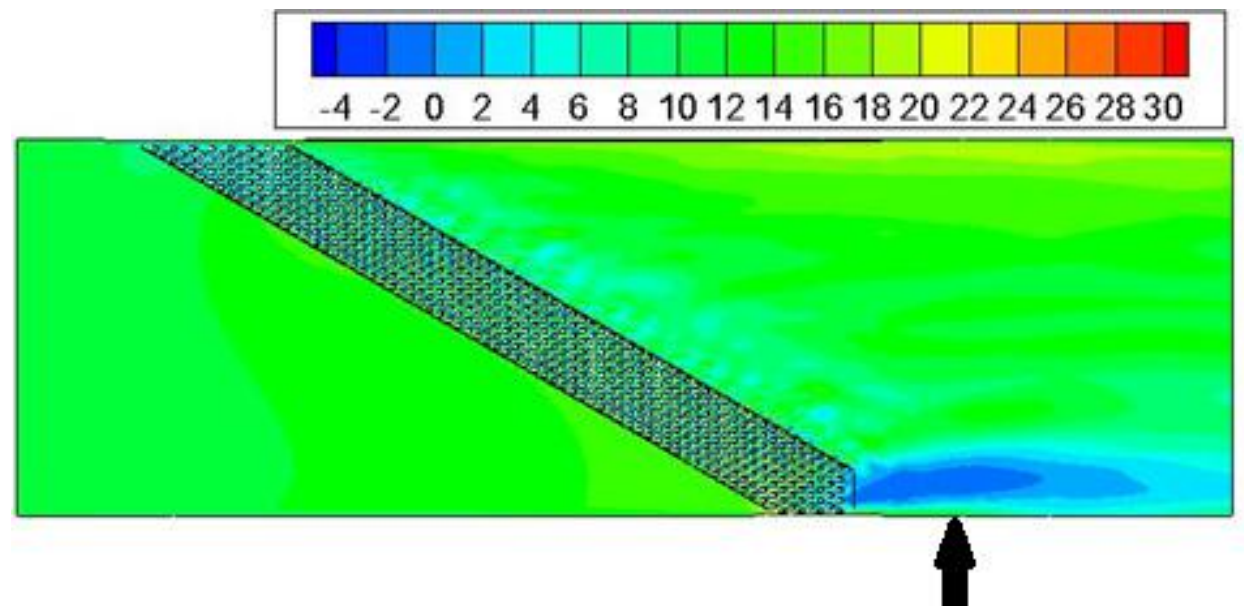

Figure 2.3.3: Velocity contour (ft/s) of the air flow through one section of the heat exchanger. The black arrow marks the location of the end of the splitter plate. 
Streamwise velocity, vertical velocity, turbulent kinetic energy, and turbulent dissipation will be recorded 12" downstream of the splitter plate for a new inlet boundary condition for the full tunnel IRT simulations. This location matches with the beginning of Corner D seen in Fig. 2.3.4. Figure 2.3.4 is a photograph with a side view perspective of the exit of the heat exchanger; the inner wall around Corner D can be seen in the background. The 12" past the splitter plate (the brown horizontal plate at the bottom of the Fig. 2.3.4.) match with the start of the turn in Corner D (the white part of inner wall seen in the background in Fig. 2.3.4). As the two-dimensional simulation encompasses only one section of the 2012 heat exchanger, the full tunnel inlet will incorporate six profiles from the two-dimensional heat exchanger simulation stacked vertically.

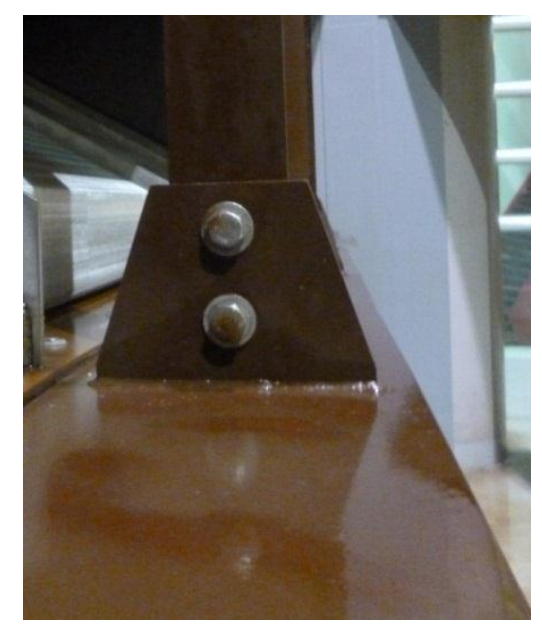

Figure 2.3.4. Photograph of the exit of the heat exchanger showing the splitter plate that separates the different sections of the 2012 heat exchanger. In the background the inner tunnel wall can be seen starting its turn for Corner D. The support beam and the bolts are not modeled in the 2-D 2012 heat exchanger simulation.

\subsection{Full Tunnel with Turning Vanes}

To obtain new inlet boundary conditions upstream of the spray bar, a RANS simulation was computed from the exit of the 2012 heat exchanger, around the turning 
vanes in Corner D, and up to the spray bars. Having a quarter-tunnel simulation of this section of the tunnel would be inappropriate because of the lack of horizontal symmetry around Corner D and the lack of vertical symmetry from the six sections of the heat exchanger. As such, the simulations needed to be performed with full three-dimensional tunnel domains. Figure 2.4.1 displays a top view of the computational domain developed for the simulation around Corner D. The air flow moves from the top of Fig. 2.4.1, at the exit of the heat exchanger, around the turning vanes and then to the right towards the outlet which is located 25 " before the spray bars. The finest grid spacing is located around and immediately downstream of the turning vanes (Fig. 2.4.2) to capture the boundary layer and wake generated by the vanes. The domain is coarsened upstream and downstream of the vanes to ease computational demand, but was tailored to ensure it sill properly model the turning vane wakes. The computational grid also becomes finer at the center of the tunnel near the outlet to match the computational domain of the settling chamber simulation which has fine grid spacing at the tunnel center for the spray bars and vertical struts. The Corner D computational domain has a total of 11.8 million grid points. 


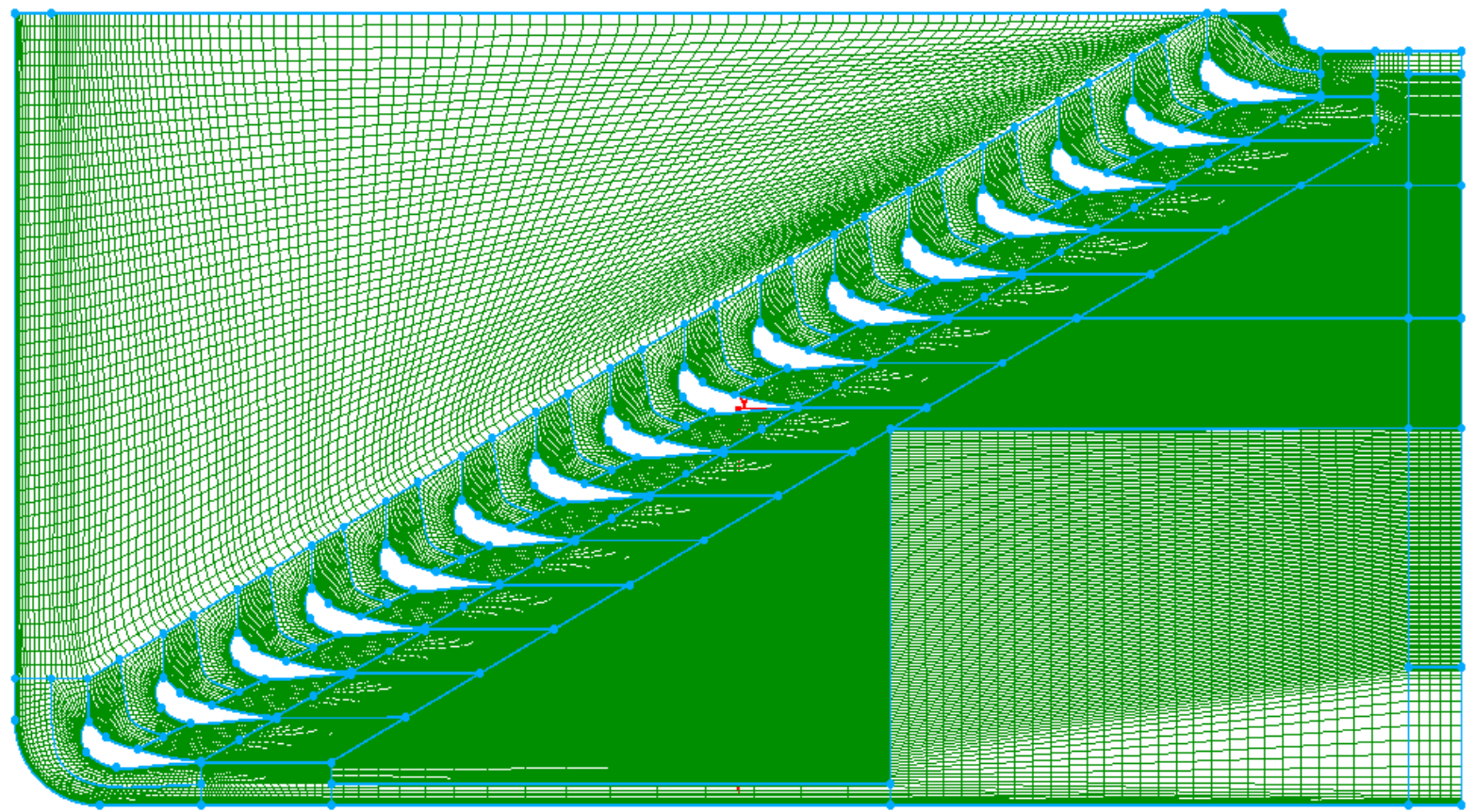

Figure 2.4.1. Top view perspective of the computational grid for the full tunnel at Corner D. The finest grid spacing is around and immediately downstream of the turning vanes while the grid coarsens upstream and downstream of the vanes where the flow is relatively calm.

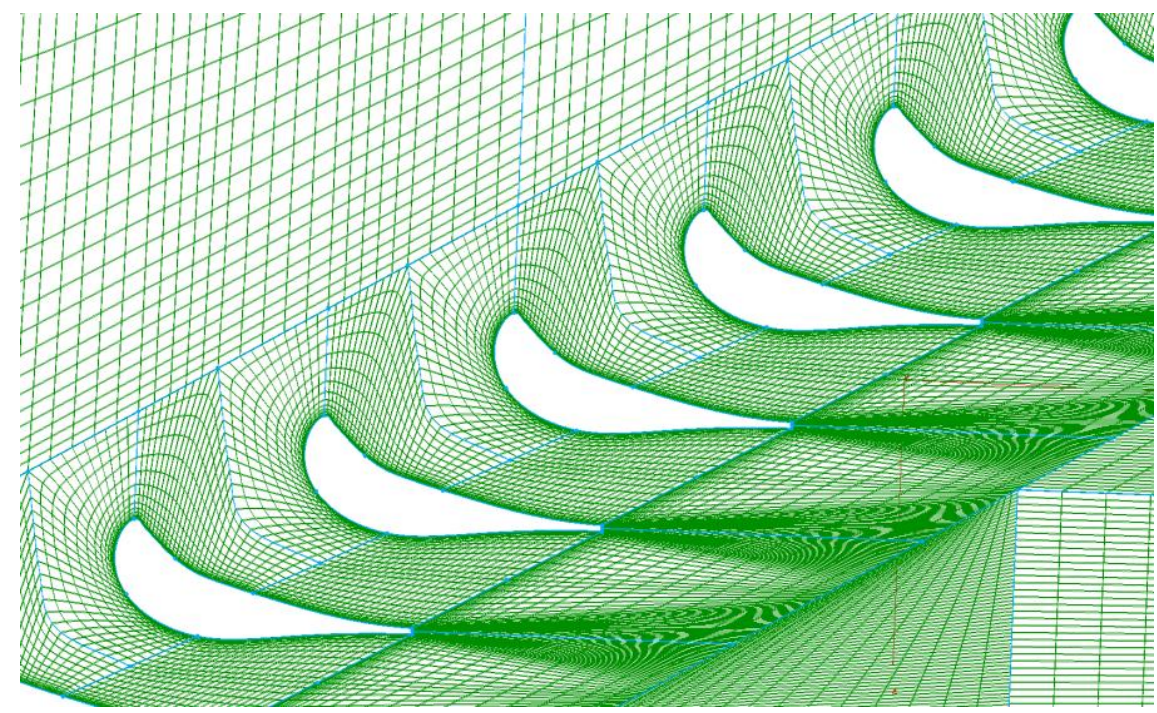

Figure 2.4.2. Close up of the computational domain around the turning vanes. 
A profile generated by the two-dimensional simulation of the heat exchanger shown previously is used to generate the inlet boundary for the simulation around Corner D. Since the heat exchanger simulation only included one section of the heat exchanger, the profile was arranged vertically six times and assumed to be uniform in the horizontal direction to encompass the entire tunnel. Figure 2.4.3 displays contours of velocity and turbulent kinetic energy of the inlet looking upstream (i.e. the right side represents the inner tunnel wall). The low velocity regions represent the lower region of a heat exchanger section that generates from the wake behind the cooling pipes and the high velocity region represents the upper region of a heat exchanger section. This high velocity gradient creates a high amount of turbulence at a splitter plate which is shown by higher regions of turbulent kinetic energy seen in Fig 2.4.3b. This non-uniform flow field at the heat exchanger exit greatly differs from the previous IRT simulations. As such, its effect on the flow throughout the NASA IRT is a main focus of the 2012 tunnel simulation.

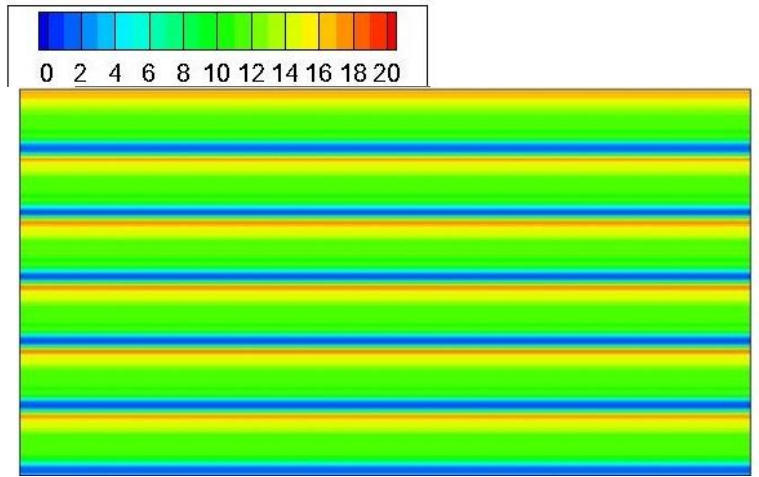

(a)

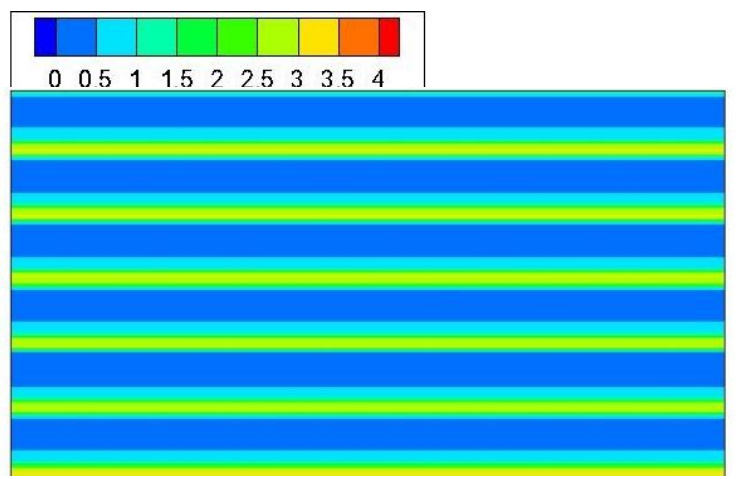

(b)

Figure 2.4.3. Contours at the exit of the 2012 heat exchanger and inlet conditions for Corner D simulations: (a) velocity magnitude (ft/s) and (b) turbulence $\left(\mathrm{ft}^{2} / \mathrm{s}^{2}\right)$. 
Figure 2.4.4 illustrates the flow progression through Corner D by showing multiple vertical slices of velocity and turbulent kinetic energy. The inlet, immediately upstream of the turning vanes, immediately downstream of the turning vanes, further downstream of the vanes, and the outlet were chosen for slice locations. The velocity increases after the turning vanes due to the converging geometry of the vanes and the reduction in the width of the tunnel after Corner D. Wakes can be seen downstream of the turning vanes as vertical lines of low velocity. The turbulent kinetic energy generated by these vertical wakes can be seen interacting with the horizontal lines of high turbulent kinetic energy generated by the sections of the 2012 heat exchanger in Fig. 2.4.4b. The inner section of the turn demonstrates much higher turbulent kinetic energy than the outer section of the turn due to the outer turn having more area to dissipate the turbulence. The differences between the inner and outer section of the turn can be clearly seen in the outlet contours (Fig. 2.4.5).

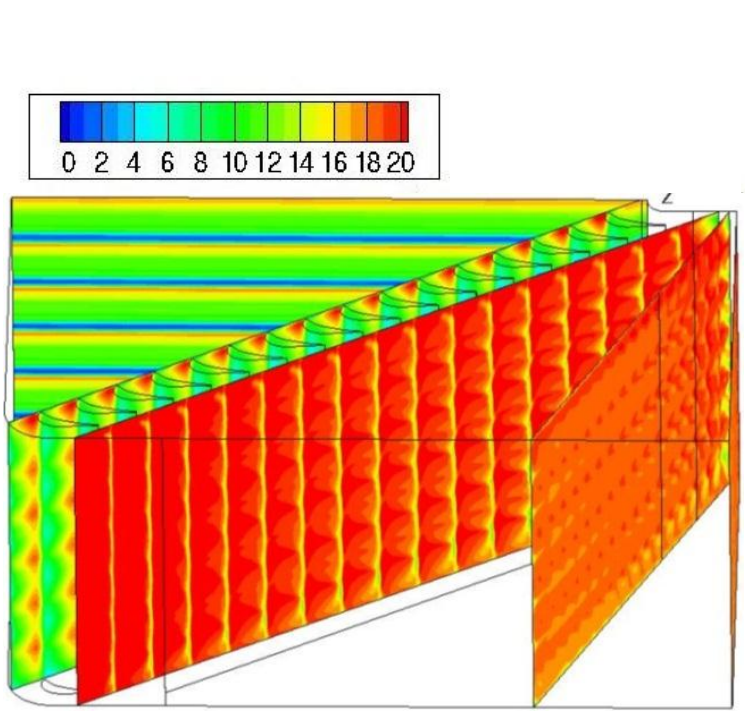

(a)
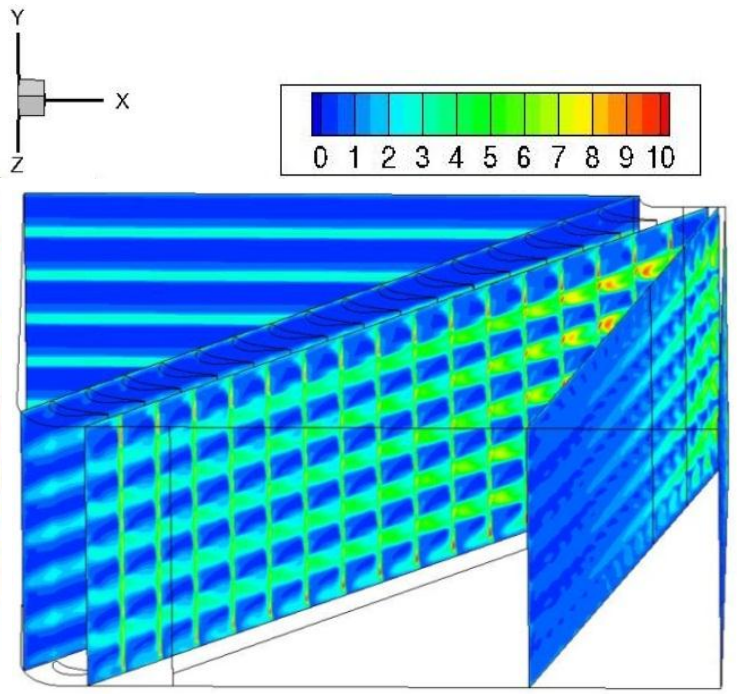

(b)

Figure 2.4.4. (a) Velocity contour slices in $\mathrm{ft} / \mathrm{s}$ and (b) turbulence contour slices in $\mathrm{ft}^{2} / \mathrm{s}^{2}$ of Corner D simulation with 2012 heat exchanger inlet. 


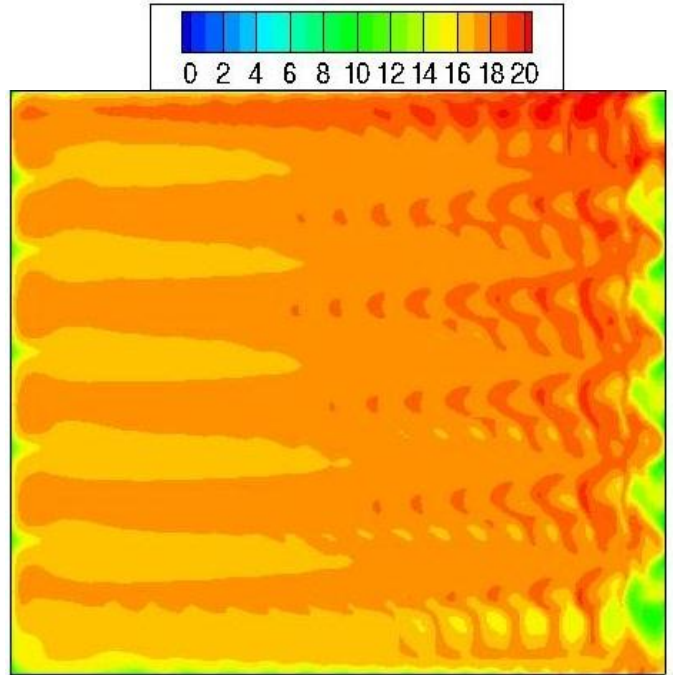

(a)

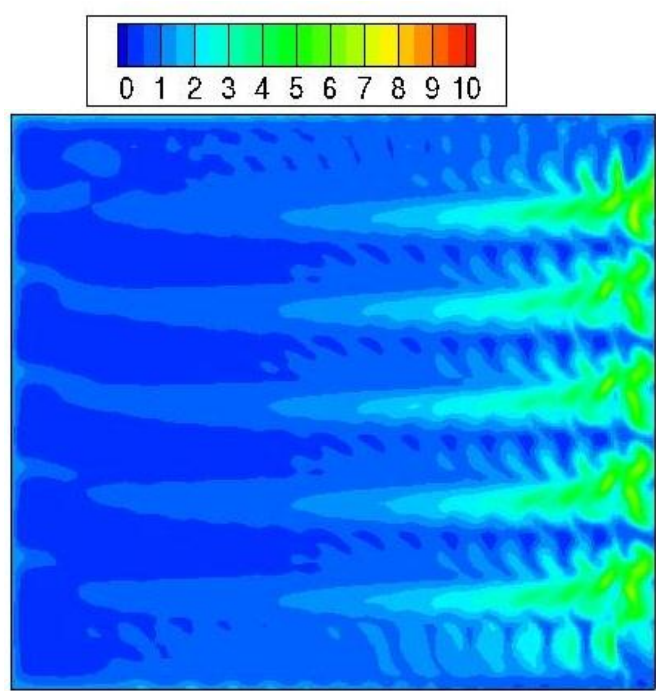

(b)

Figure 2.4.5. Contour slices of the outlet in the Corner D full tunnel simulation and is located 25 " before the spray bars and vertical struts: (a) velocity magnitude ( $\mathrm{ft} / \mathrm{s})$ and (b) turbulence $\left(\mathrm{ft}^{2} / \mathrm{s}^{2}\right)$.

Figure 2.4.5 shows contours of velocity in $\mathrm{ft} / \mathrm{s}$ and turbulent kinetic energy in $\mathrm{ft}^{2} / \mathrm{s}^{2}$ for the outlet of the Corner D simulation which is 25 " upstream of the spray bars. This location corresponds to a new inlet for the full tunnel simulation from the spray bars to the tunnel test section. The contours are looking upstream, i.e. the right side represents the inner wall of the tunnel. The inner section of the tunnel creates much higher turbulent kinetic energy than the outer section of the tunnel because of the shorter distance in which air flow has to travel from the heat exchanger to the outlet at the inner section. The larger distances provide the air flow with more time to dissipate the higher kinetic energy generated by the 2012 heat exchanger. Some of the wakes from the inner turning vanes persist and can be seen as vertical lines of high turbulent kinetic energy. However, the majority of the turbulence at this location seems to be generated by the 2012 heat 
exchanger, especially downstream of an intersection between two heat exchanger sections which explain the five distinct horizontal areas of high turbulent kinetic energy.

The pitch and yaw flow angles were calculated using the streamwise, spanwise and vertical velocities predicted at the Corner D outlet to compare with experimental measurements $^{14}$ (Fig. 2.4.6). The vertical rakes were located 88", 195.5", and 263"from the inner wall. The simulated angles are shown as continuous lines and the experimental data is shown as individual data points. The pitch angles show the highest difference between the simulation and experiments. Experiments indicate pitch angles that vary between -5 and 5 degrees depending on the vertical rakes locations. However, the predictions yield much lower flow pitch angles and thus are not properly capturing the vertical angles of the flow direction.

In contrast, the yaw angles match up exceptionally well with both measurements and predictions displaying high fluctuations along the vertical rake of consistent magnitude and wavelength (where the latter is consistent with the vertical spacing between heat exchanger segments). Rakes farther from the inner wall show lower amplitudes in yaw angle variation which is consistent with the decay in these variations owing to the longer flow paths. The vertical rake comparisons demonstrate that the simulations are capturing some of the flow physics (yaw angles) but there is still room for improvement (pitch angles). Since the wavelength of the pitch angle is on the order of the entire cross-sectional height, the cause for these variations may lie upstream of the heat exchanger. 


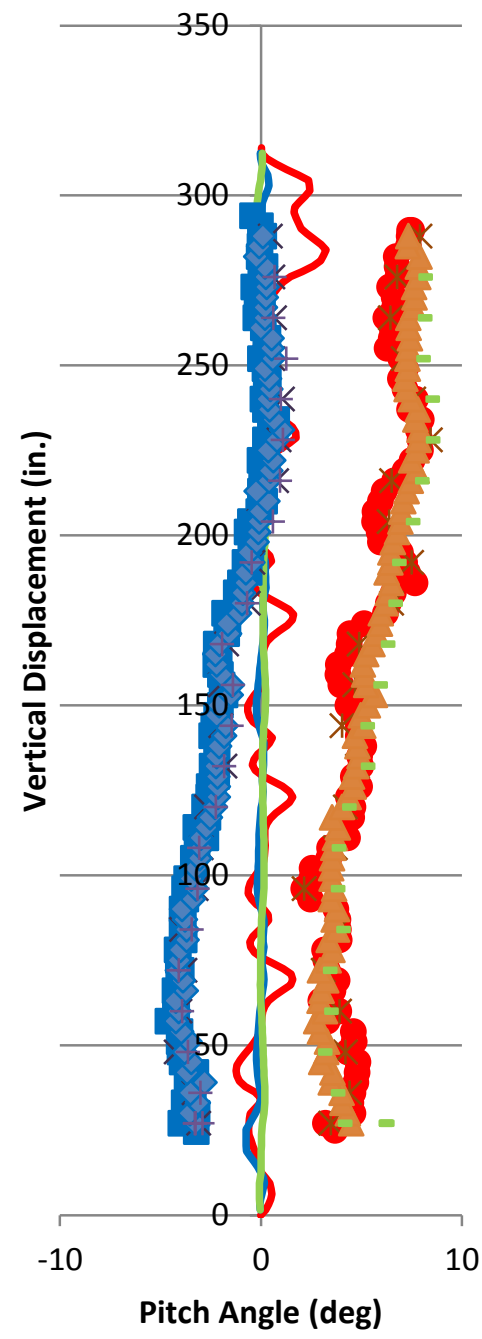

(a)

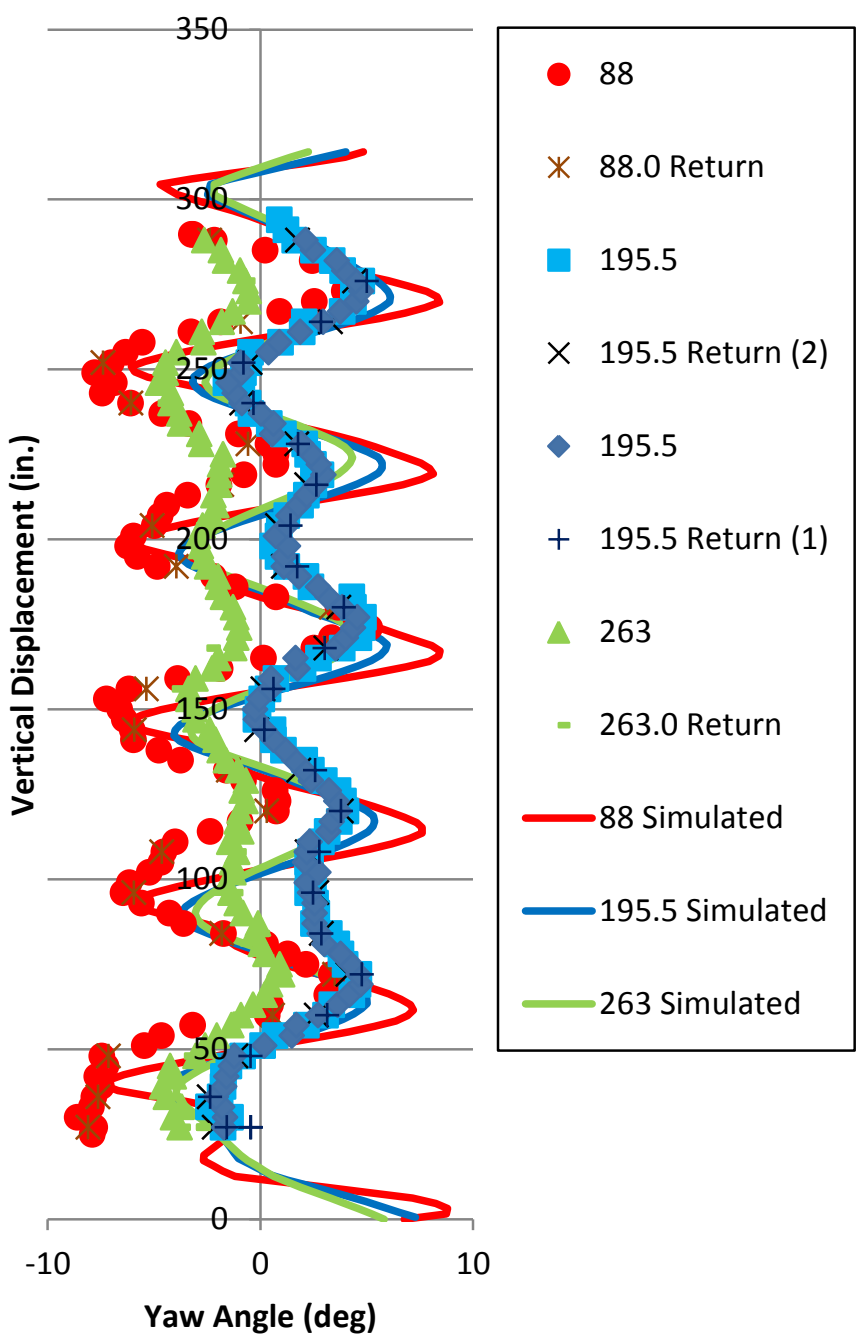

(b)

Figure 2.4.6. (a) Pitch angle and (b) yaw angle comparisons between experiments ${ }^{14}$ and simulations along three different vertical rakes located upstream of spray bars at the Corner D simulation outlet. The legend gives the distance in inches from the inner wall.

As mentioned above, the computational domain used for the previous quartertunnel simulations were expanded to encompass the entire NASA Glenn IRT due to the lack of flow symmetry. Incorporating the entire tunnel greatly increased the number of cells, e.g. the computational domain for a tunnel with additional vertical struts and no active jets has 11 million grid points. The turbulent kinetic energy from the spray bars to the test section can be seen in Fig. 2.4.7. The contour has a side view of a slice at the 
center of the tunnel. The turbulent kinetic energy from the spray bar and vertical strut wakes can be seen converging towards the center of the test section which was seen previously in the 2000 and 2009 tunnel configuration simulations.

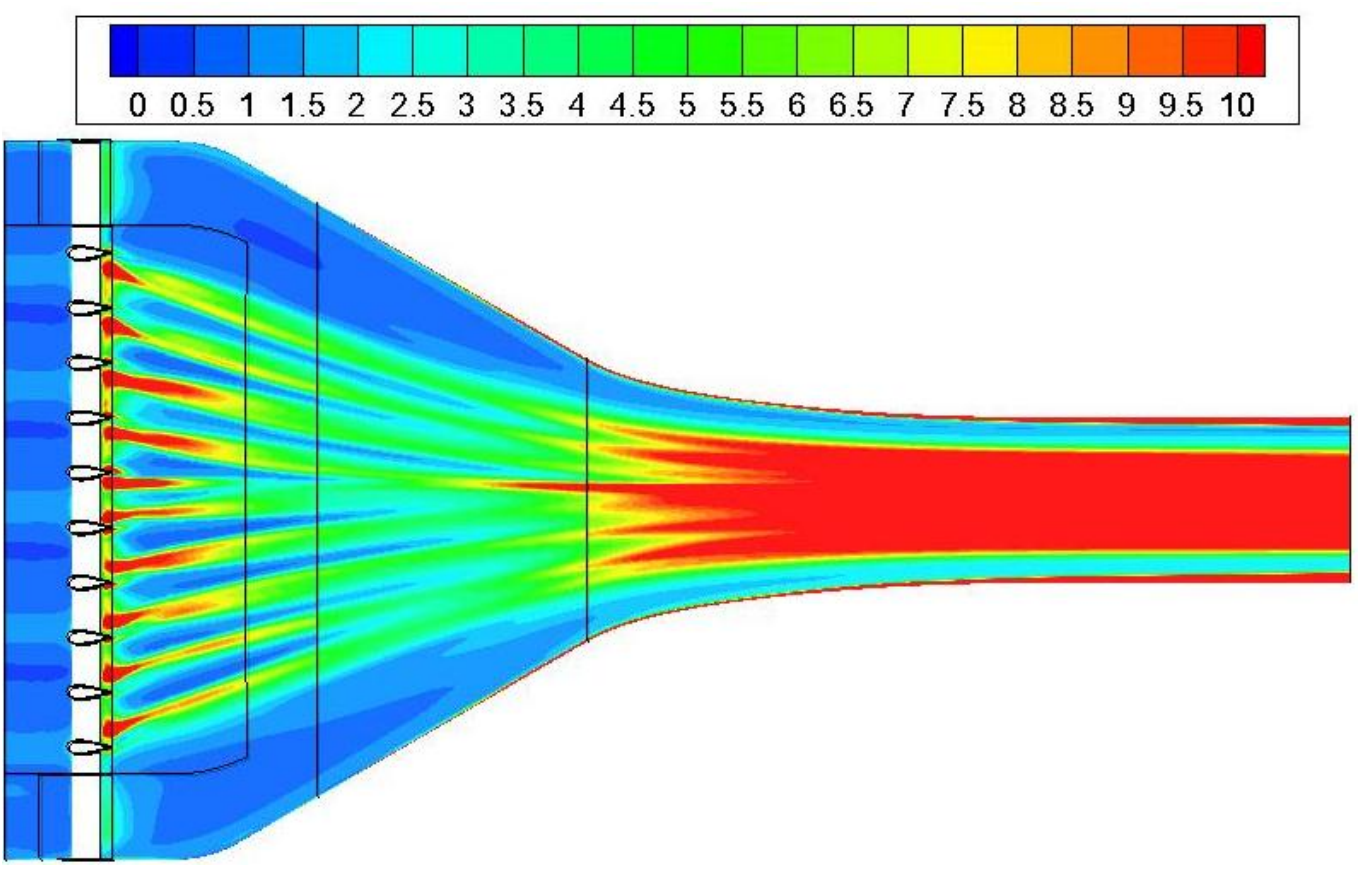

Figure 2.4.7. Contour slice of turbulent kinetic energy in $\mathrm{ft}^{2} / \mathrm{s}^{2}$ at the center of the tunnel for 2012 tunnel configuration without jets.

A contour of the turbulent length scales can be seen in Fig. 2.4.8. The turbulent length scales are calculated by ${ }^{11}$,

$$
l=\frac{k^{\frac{3}{2}}}{\varepsilon}
$$

The largest flow structures are upstream of the spray bars and struts. The spray bars and struts break up these large flow scales as the flow moves past. The smallest flow structures are immediately downstream of the spray bars and struts and show scales that are on the order of 1 " or less. The length scales grow as the flow travels through the 
tunnel contraction and increase in velocity and reach values of approximately one foot at the test section.
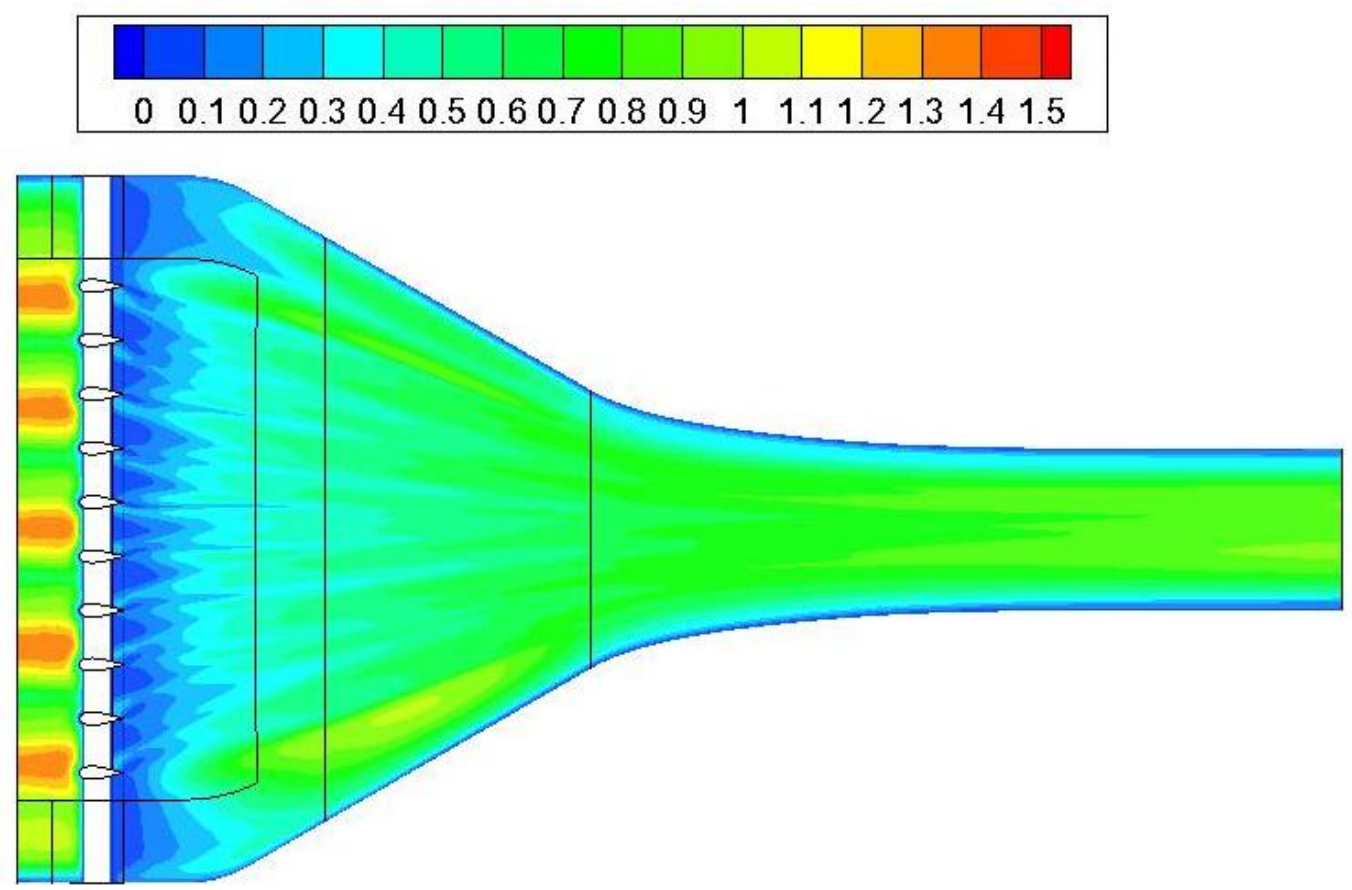

Figure 2.4.8. Contour slice of turbulent length scale in $\mathrm{ft}$ at the center of the tunnel for 2012 tunnel configuration without jets.

Vertical slices of turbulent kinetic energy from the spray bars to the test section of the 2012 IRT with vertical struts but without any active jets are shown in Fig. 2.4.9. The turbulence generated from the 2012 heat exchanger persists and can be seen interacting with the turbulence produced by the wakes of the spray bars and vertical struts. The inner section of the tunnel is shown to consistently have higher turbulent kinetic energy than the outer section due to the flow's turn around Corner D. The turbulent kinetic energy is shown to increase as the air flow accelerates through the tunnel contraction and towards the test section which is similar to the previous quarter-tunnel simulations of the 2000 and 2009 tunnel configurations. 


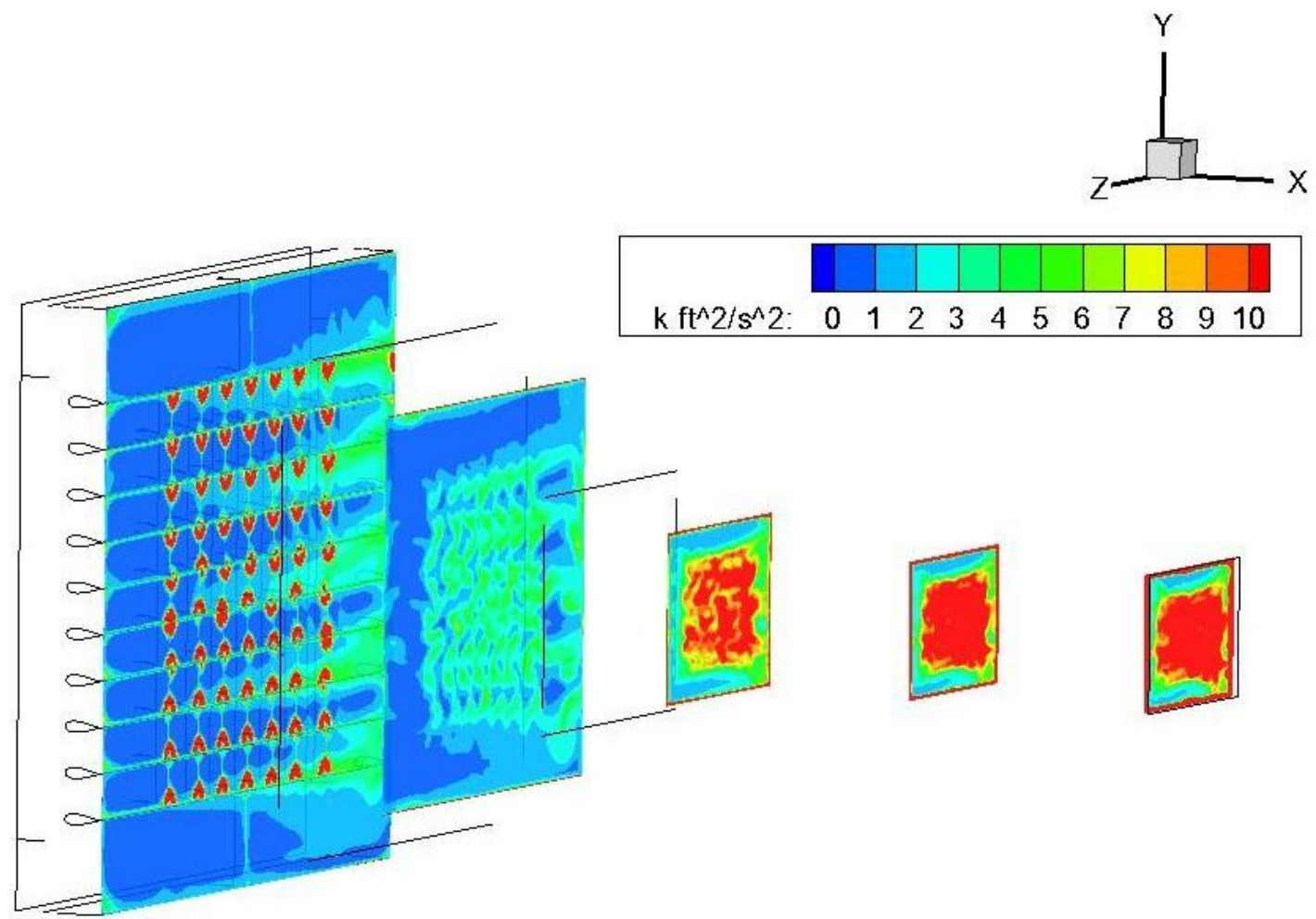

Figure 2.4.9. Contour slices of turbulence in $\mathrm{ft}^{2} / \mathrm{s}^{2}$ starting at the spray bars to the test section for 2012 tunnel configuration without jets.

Even though the turbulent kinetic energy is increasing as the flow moves through the contraction, the intensity of the turbulence relative to the fluid speed actually decreases. This can be seen in the slices of normalized velocity fluctuations in Fig. 2.4.10. The velocity fluctuations are normalized by the mean streamwise velocity by,

$$
u_{r m s}{ }^{*}=\frac{u_{r m s}}{u_{\text {mean }}}
$$

where $u_{\text {mean }}$ is the average streamwise velocity for the given contour slice. The scale is set to an exponential scale to better visualize the changes along the tunnel. The highest relative turbulence is immediately downstream of the spray bars. The relative velocity fluctuations dramatically reduce as the flow increases in velocity through the tunnel contraction (which is the purpose of a tunnel contraction). 

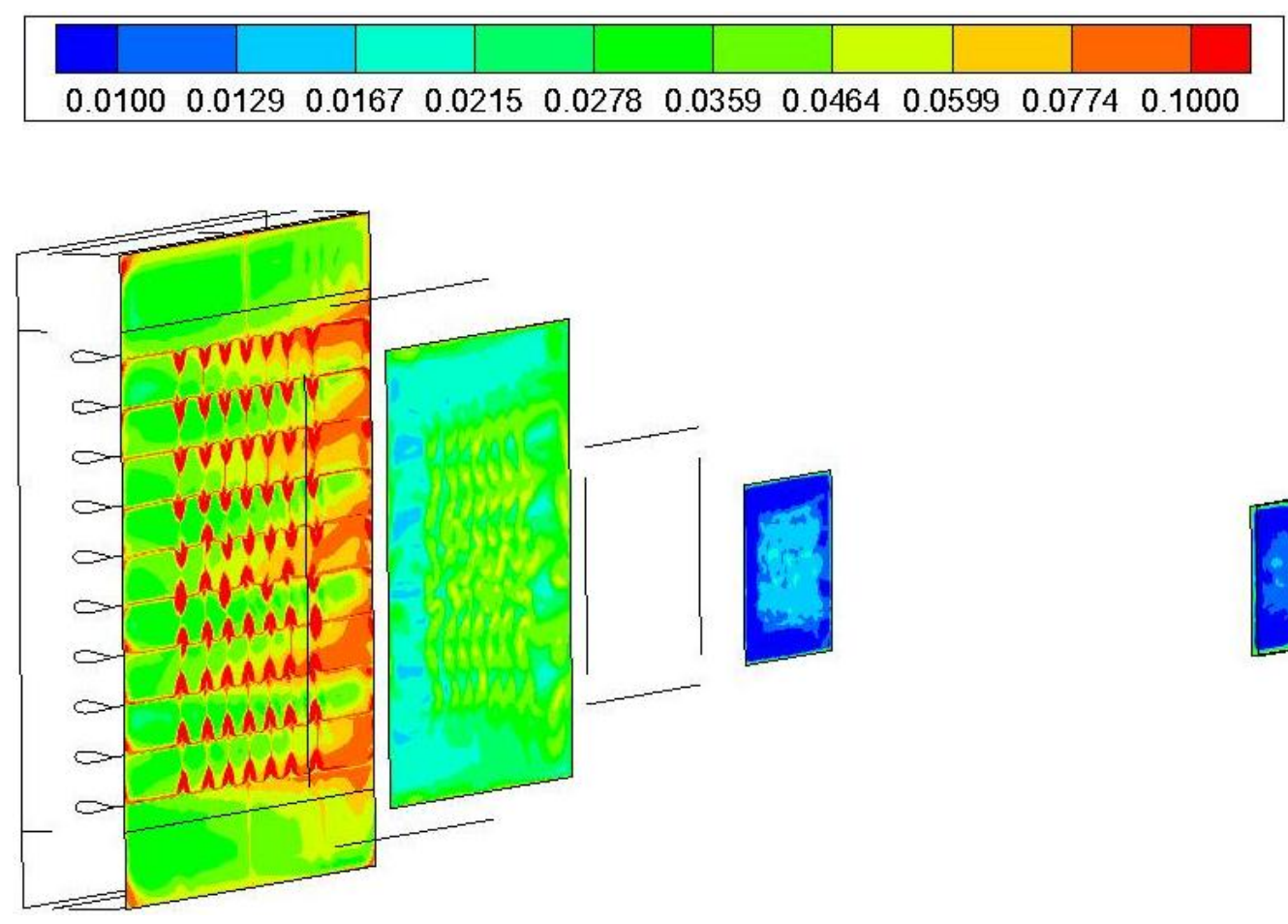

Figure 2.4.10. Contour slices of velocity fluctuations normalized by the mean streamwise velocity for the given slice. The slices are along the 2012 IRT configuration.

Figure 2.4.11 displays the turbulent kinetic energy at the test section. The center of the test section again illustrates relatively high turbulence at the center due to the spray bars and vertical struts. However, the magnitude of the turbulent kinetic energy is higher than the predictions of the 2000 and 2009 IRT simulations, due to the additional turbulence generated by the 2012 heat exchanger. Table 2.4.1 displays the average turbulent kinetic energy at the test section excluding the high values at the walls for the 2000, 2009, and 2012 simulations. All three cases do not have active jets running, but as mention previously, the 2009 case includes additional vertical struts which were included in the 2012 simulation. The 2012 tunnel configuration was simulated and run experimentally without the additional vertical struts, but the droplet dissipation was shown to be unsatisfactory. This required the additional vertical struts to be installed. The 
turbulent kinetic energy in the 2012 tunnel configuration increased by about 36 percent from the 2009 tunnel configuration as shown in Table 2.4.1. The additional turbulence will assist in water droplet distribution for the 2012 IRT, but may cause undesirable flow features at the test section. Experimental testing will be required to ensure that the new level of turbulent kinetic energy at the test section is acceptable.

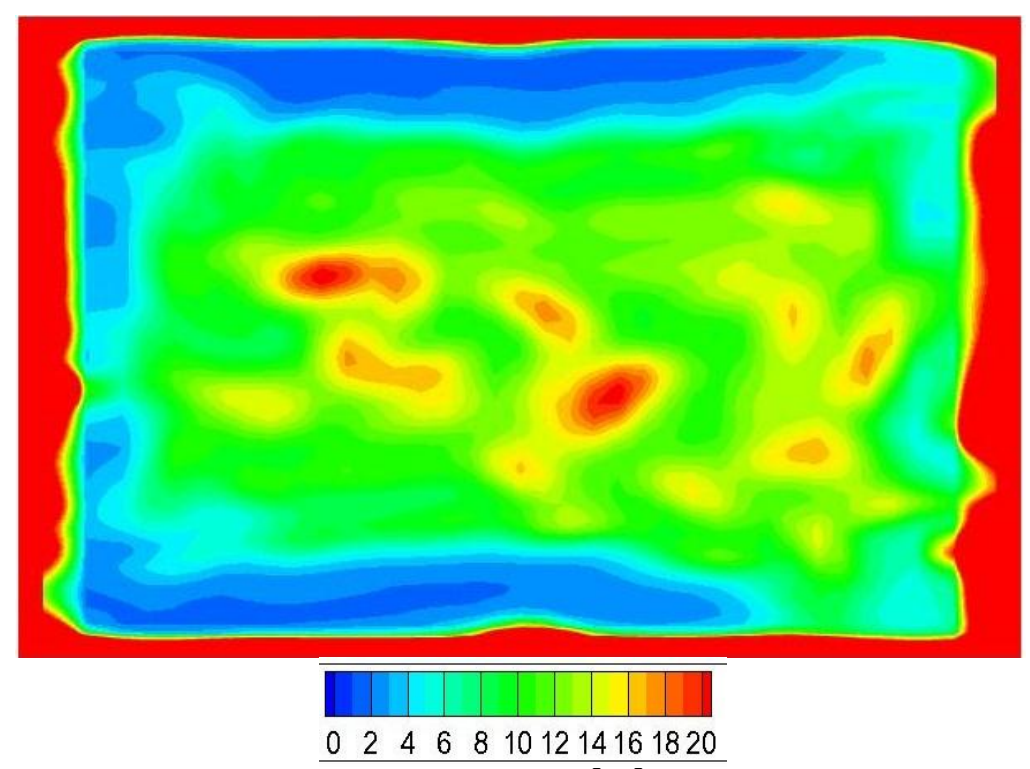

Figure 2.4.11. Turbulent kinetic energy contour in $\mathrm{ft}^{2} / \mathrm{s}^{2}$ at the test section for the 2012 tunnel configuration without jets.

\begin{tabular}{|c|c|c|c|}
\hline Source & $\begin{array}{c}\text { Tunnel } \\
\text { Configuration }\end{array}$ & Jets & $\mathbf{k}_{\mathbf{a v}}\left(\mathbf{f t}^{2} / \mathbf{s}^{\mathbf{2}}\right)$ \\
\hline $\begin{array}{c}\text { Present } \\
\text { Predictions }\end{array}$ & 2000 & No Jets & 3.00 \\
\hline $\begin{array}{c}\text { Present } \\
\text { Predictions }\end{array}$ & $\begin{array}{c}2009 \\
\text { vertical struts installed }\end{array}$ & No Jets & 6.44 \\
\hline $\begin{array}{c}\text { Present } \\
\text { Predictions }\end{array}$ & $\begin{array}{c}2012 \\
\text { new HX also installed }\end{array}$ & No Jets & 8.75 \\
\hline
\end{tabular}

Table 2.4.1 Average test section kinetic energy comparison between 2000, 2009, and 2012 tunnel configurations. 


\section{Chapter 3. Liquid Water Concentration and Droplet Trajectories}

\subsection{Methods}

Water droplet trajectories within the NASA Glenn IRT simulations were computed with an unsteady Lagrangian discrete phase model. A Lagrangian model tracks each individual particle. It differs from a Euleran approach that assumes the particles act as a continuum. The droplets in the current study were assumed to be spherical particles with a constant density of water. The particle equation of motion for this model is,

$$
\frac{d v_{i}}{d t}=\frac{F}{m}+g
$$

The force $(F)$ on the particle can include many different components, e.g., drag, lift, added mass, fluid stress, collisions, Brownian diffusion, etc, ${ }^{15}$. Brownian and thermophoresis occur at the molecular level and can be neglected because the water droplet diameters considered were large, being on the order of microns (10-40 microns). Lift, added mass, history, and fluid-stress forces can be assumed insignificant for high ratios of particles density to surrounding fluid density (e.g. water droplets in air). As such, the current study assumes that the drag force will comprise the majority of the surface force on the particle and was implemented with a sphere drag law ${ }^{16}$. This is reasonable because the Weber number of these drops was always much less than unity. The Weber number is defined as,

$$
W e=\frac{\rho_{f}(u-v)^{2} d}{\sigma}
$$

where $\sigma$ is the surface tension forces, $\rho_{f}$ is the density of the fluid, $u$ is the fluid velocity, $v$ is the particle velocity, and $d$ is the particle density. 
A discrete or discontinuous random walk model (DRW) was employed to allow the strength of the air flow turbulent kinetic energy to affect the trajectories of the water droplets. The DRW assumes that the air flow velocity is the sum of the mean and fluctuating components as shown,

$$
u_{i}=\bar{u}_{i}+\zeta u_{i, r m s}^{\prime}
$$

The $\zeta$ in this equation is a random number with a Gaussian distribution and a mean value of zero. Having a random number generator will replicate the unpredictability seen naturally in high turbulent flows. The root mean square of the velocity fluctuations can be obtained from the turbulent kinetic energy derived from the Menter SST model,

$$
u_{i, r m s}^{\prime}=\sqrt{\frac{2 k}{3}}
$$

Areas of high turbulence intensity will see stronger velocity fluctuations which will cause the droplets to disperse faster ${ }^{11}$.

The liquid water droplets were released in the IRT domain 4" downstream of the spray bars to be consistent with the air flow from the jets that initiate 4 " downstream. Since the water droplets are released and tracked as individual particles, any break-up and coalition was not considered. The initial particle velocities were based off droplet results recorded from experiments by Bulzan ${ }^{9}$ at $3.94 "(10 \mathrm{~cm})$ downstream of the nozzle exit seen in Fig. 3.1.1. This initial radial profile was rotated every 20 degrees until a full 360 degrees profile was created to release the liquid water droplets within the three dimensional NASA Glenn IRT simulations. The initial radial water droplet concentration was based off the given water mass flow rate in the experiment and by assuming the concentration distribution is similar to the radial velocity profile. 


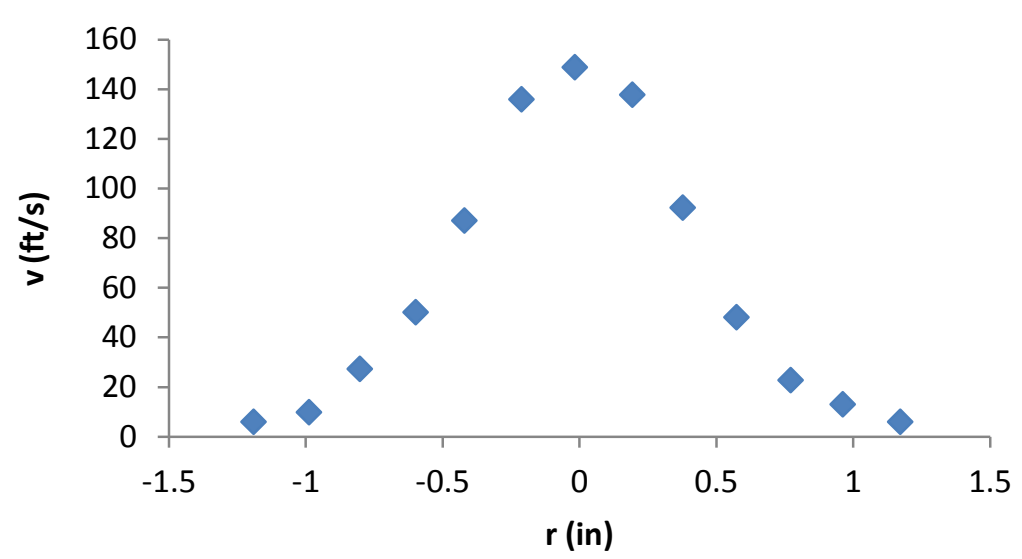

Figure 3.1.1. Radial profile of droplet velocities 3.94" $(10 \mathrm{~cm})$ downstream of nozzle exit from experiments ${ }^{4}$. This profile is utilized as the initial velocities of the droplets being released 4" downstream of the nozzle exit.

A single jet was simulated with $18 \mu \mathrm{m}$ size droplets being released using the three dimensional 4" profile to test its fidelity and to decide if two-way coupling is required. The air flow was analyzed from simulations without droplets and with droplets and twoway coupling to decide if the particles are affecting the air flow at locations past 4" downstream of the nozzle exit. Figure 3.1.2 shows the results of the centerline air flow velocity between the two simulations and experiments by Bulzan'. There are no noticeable differences between simulating the air flow without any particles and simulating particles with two-way coupling which determines that the particles are not affecting the air flow. As such, utilizing one-way coupling and calculating the droplet trajectories separately once the air flow field is determined is a reasonable approach. 


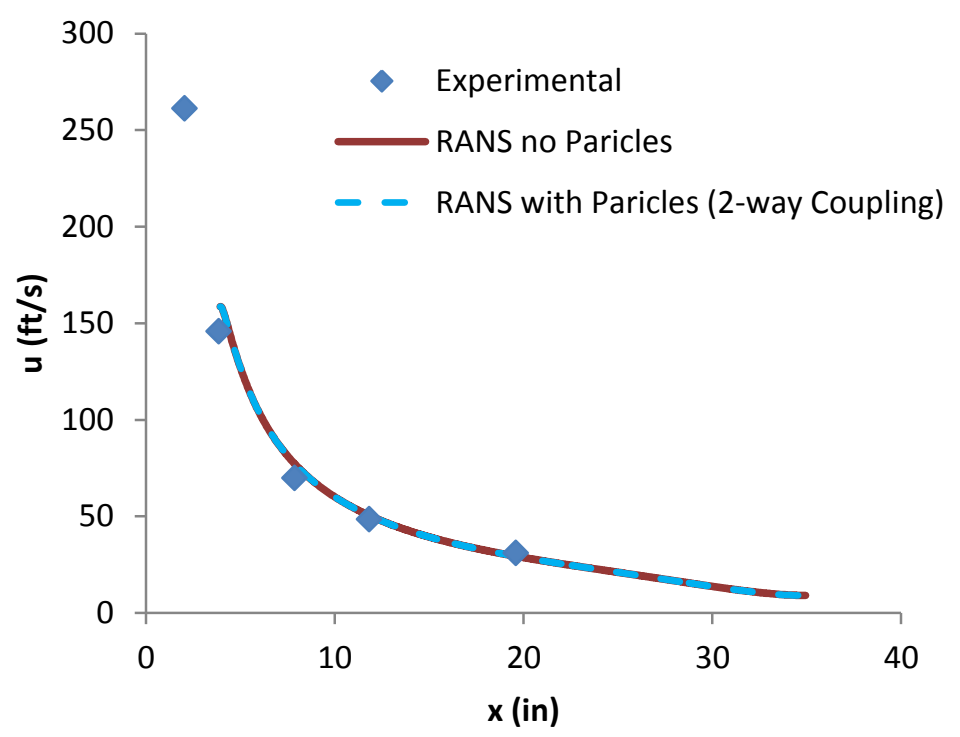

Figure 3.1.2. The centerline air flow velocity for a single water nozzle from experiments ${ }^{4}$, air flow only simulation and simulation of air flow with particles and twoway coupling.

Figure 3.1.3 displays the droplet velocities and radial locations at 7.9" $(20 \mathrm{~cm})$ and 11.8" $(30 \mathrm{~cm})$ downstream of the nozzle exit for both the current single jet predictions and experiments by Bulzan ${ }^{9}$. The average droplet velocities along the radial direction were computed from the droplets shown and are also plotted on Fig. 3.1.3. The droplet simulations seem to slightly over predict the average droplet velocity seen during experiments with the largest difference of 9 percent difference at the centerline $20 \mathrm{~cm}$ downstream of the nozzle. The differences between simulations and experiments decrease at large radial distances and as the droplets move downstream which give confidence in its implementation in the NASA IRT simulations. 


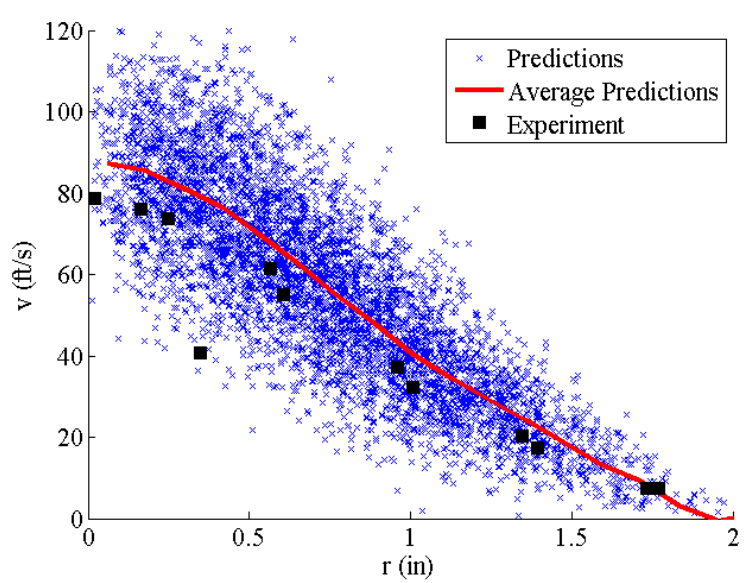

(a)

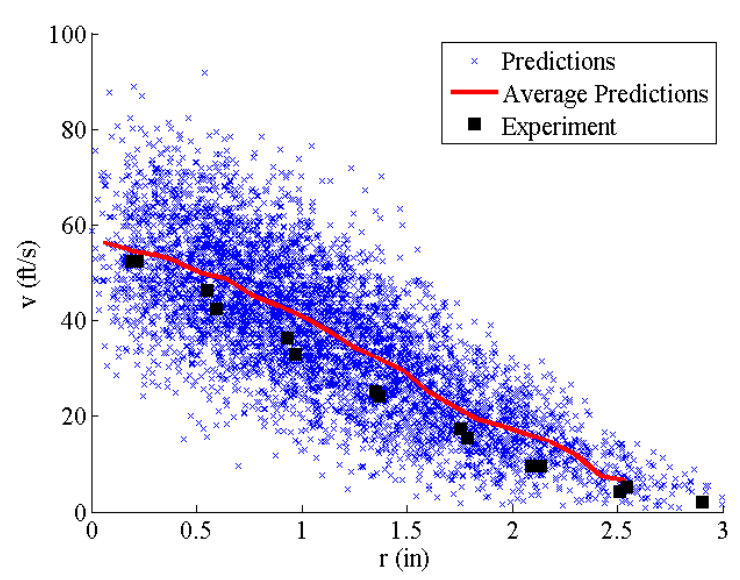

(b)

Figure 3.1.3. Droplet velocities by predictions simulation and experiments by Bulzan ${ }^{9}$ (a) 7.9" $(20 \mathrm{~cm})$ and (b) $11.2 "(30 \mathrm{~cm})$ downstream of the nozzle.

\subsection{Droplet Trajectories and Transfer Map}

The water droplet sizes were based off of a cumulative distribution function tabulated from experimental data ${ }^{4}$ obtained within the NASA Glenn IRT. The experimental data had a range of water droplet sizes with a mean volumetric diameter (MVD) of $21 \mu \mathrm{m}$. The experimental data was fitted with a Rosin-Rammler distribution using the equation seen below,

$$
C(d)=1-\exp \left[-\left(\frac{d}{d_{r r}}\right)^{\frac{1}{h_{r r}}}\right]
$$

In this expression $C(d)$ is the cumulative droplet distribution, $d$ is the droplet diameter, $d_{r r}$ is the reference diameter and corresponds to $C(d)=0.63$, and $h_{r r}$ is the spread parameter ${ }^{11}$. For the current Rosin-Rammler fit $d_{r r}=24 \mu \mathrm{m}$ and $h_{r r}=2.4$. This cumulative distribution function was converted into a probability density function and ten droplet bin sizes were determined by keeping the integral of the Rosin-Rammler fit equal between each droplet size which can be seen by the black lines in Fig. 3.2.1. Ten different 
droplet diameters were chosen to give an adequate range of droplet sizes and using the Rosin-Rammler fit allows the MVD to stay at $21 \mu \mathrm{m}$ and to match with experiments.

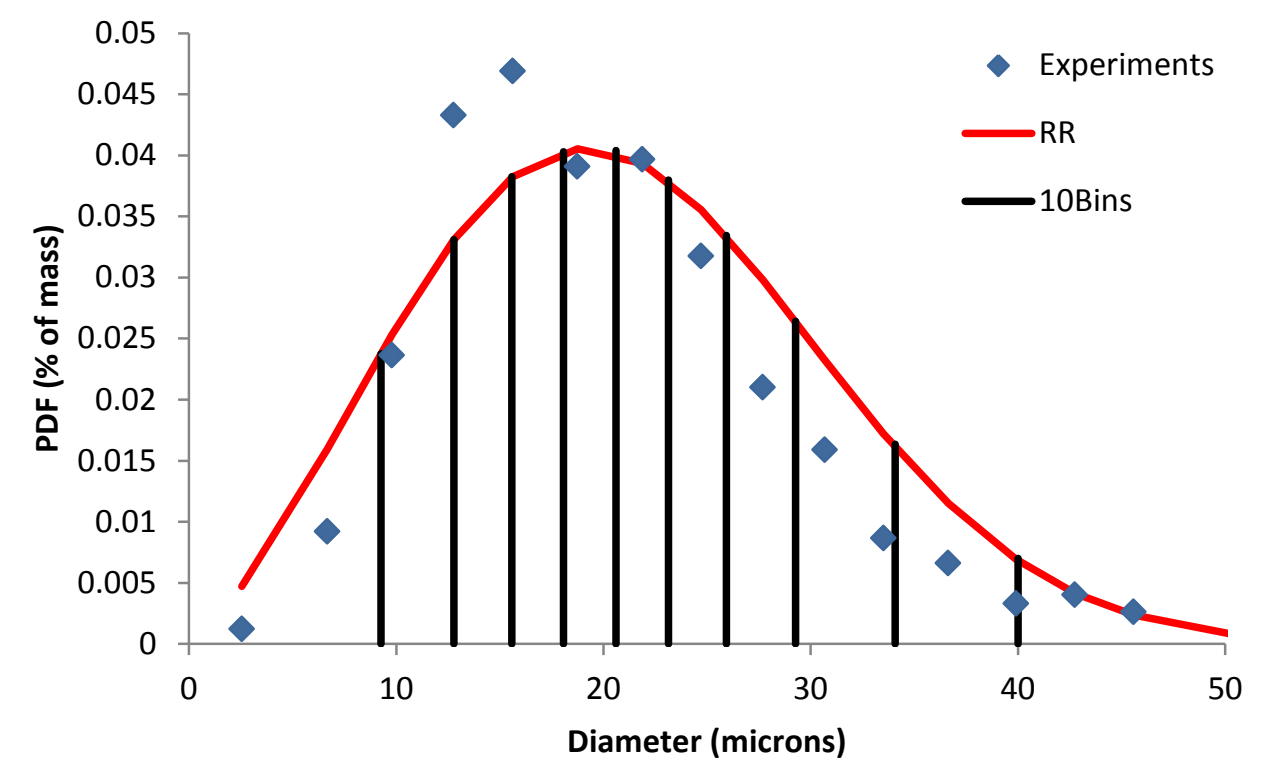

Figure 3.2.1: Diameters sizes for 10 bin distribution using a Rosin-Rammler fit of experimental data ${ }^{4}$.

A convergence study was conducted to determine the number of droplets required to obtain reasonable scatter and concentration plots at the IRT test section. This convergence study used the 2012 NASA IRT with additional struts and released droplets from the $5^{\text {th }}$ spray bar from the tunnel floor with nine jets spaced relatively evenly as seen in Fig. 3.2.2. The first simulation released and tracked 5,000 droplets per jet and the second simulation released and tracked 10,000 droplets per jet. Scatter plots at the test section of the two simulations can be seen in Fig. 3.2.3. The scatter and concentration plots are generated looking downstream, i.e. the left wall represents the inner wall of the tunnel and bottom represents the tunnel floor. The scatter plots show relatively little noticeable difference between 5,000 and 10,000 droplets per jet. 


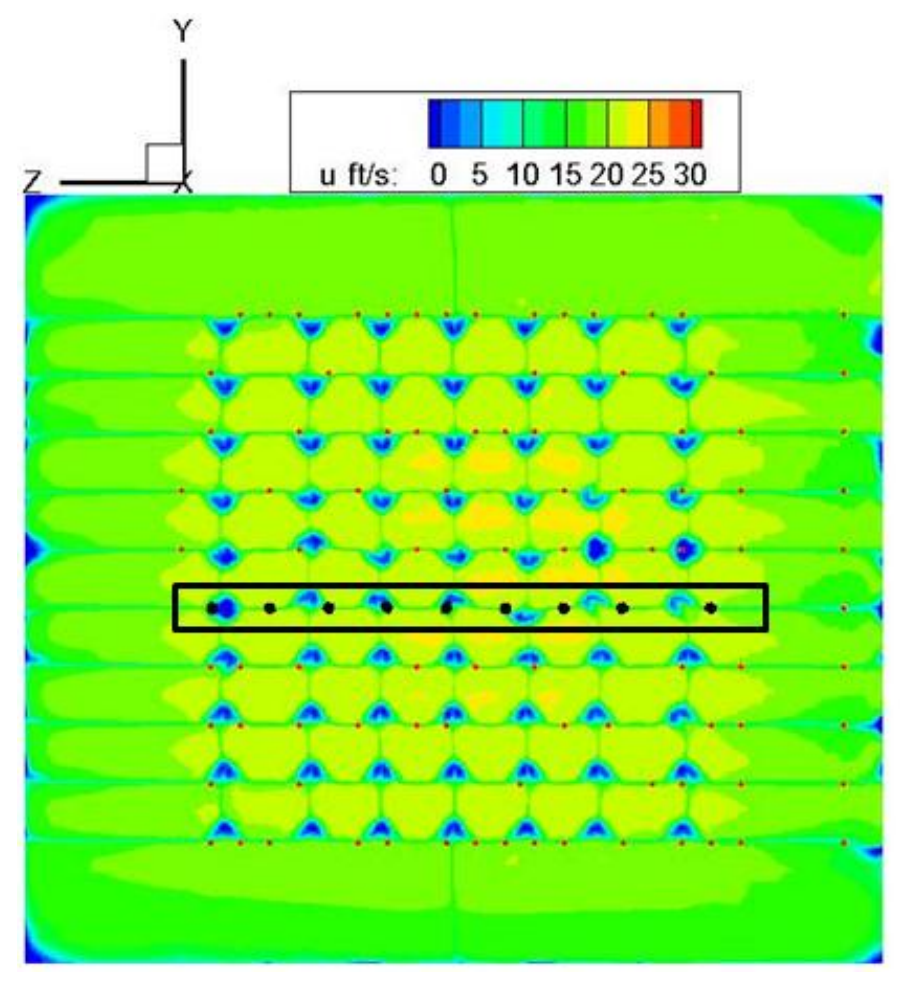

Figure 3.2.2. Velocity contour 4" downstream of the spray bars where particles are injected in the 2012 tunnel configuration. Black box shows which spray bar (bar five) used for convergence study and red spots show active jet locations where particles can be injected. As with the other air flow plots, this is imaged looking in the upstream direction, i.e. the right side represents the inner wall of the IRT.

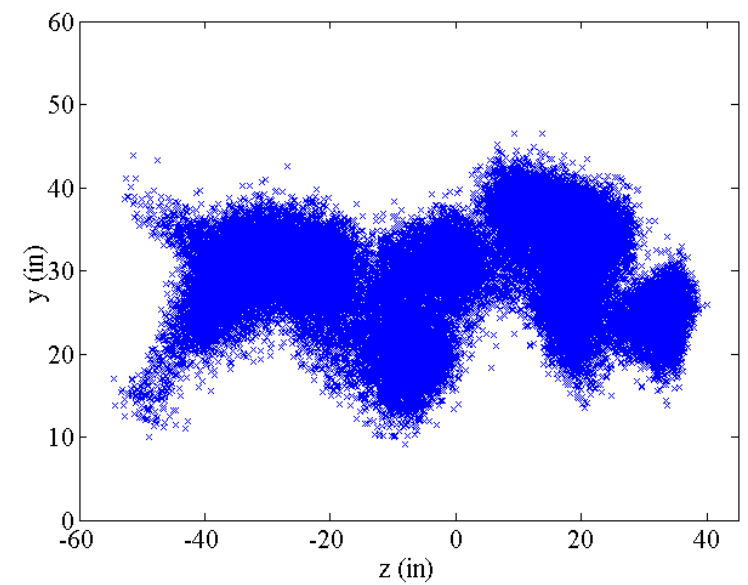

(a)

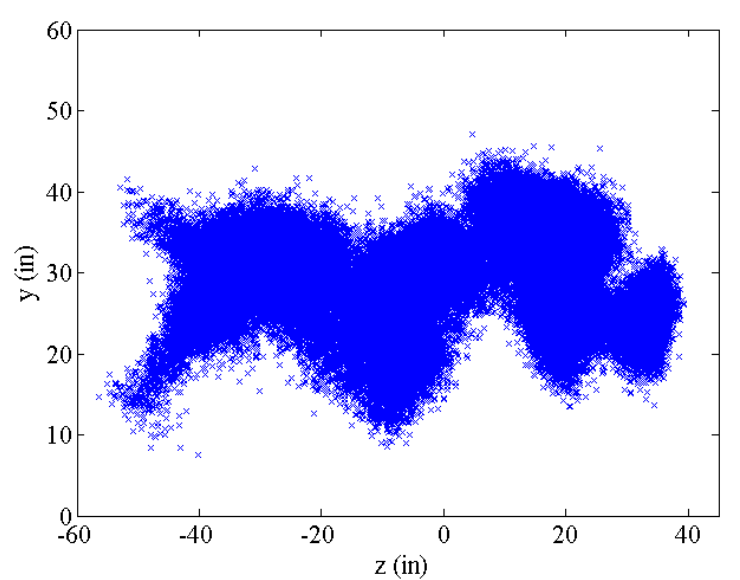

(b)

Figure 3.2.3. Scatter plots at test section of the convergence study (a) 5000 droplets (b) 10000 droplets. As with the following droplet scatter and concentration plots, these are imaged looking in the downstream direction, i.e. the left side represents the inner wall of the IRT and $\mathrm{z}=0$ represents the horizontal center of the tunnel and $\mathrm{y}=0$ represents the floor. 
Liquid water concentration plots at the test section are shown in Fig. 3.2.4 for 5,000 and 10,000 droplets per jet. The liquid water concentration uses 5.9" x 5.9" $(15 \mathrm{~cm})$ bins which correspond to the test grid that is used experimentally for calibration of the Glenn IRT. The concentration plots are calculated and normalized by the following,

$$
\alpha^{*}=\frac{\alpha}{\alpha_{a v g}}, \text { where } \alpha=\sum \frac{d^{3}}{A_{b i n} v}
$$

where $\alpha$ is the concentration, $d$ is the particle diameter, $A_{b i n}$ is the bin area and $v$ is the particle velocity. The normalization, with an average between all the bins, allows for comparison between multiple simulations. The concentration contours seen in Fig. 3.2.4 also demonstrate little noticeable difference between releasing 5,000 droplets and 10,000 droplets per jet and demonstrates that releasing 5,000 droplets per jet gives converged concentration predictions at the IRT test section.

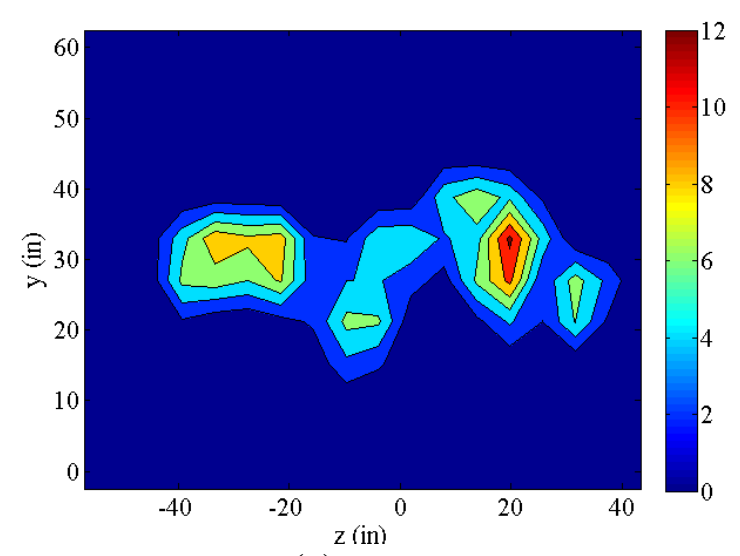

(a)

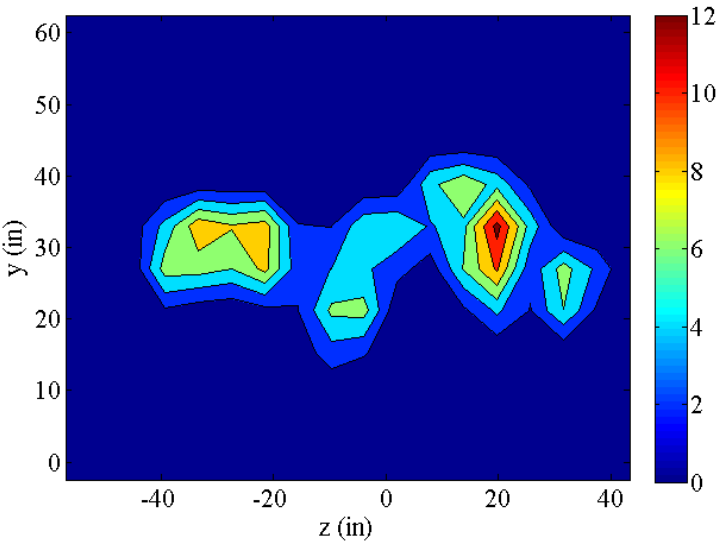

(b)

Figure 3.2.4. Concentration plots at test section of the convergence study (a) 5000 droplets and (b) 10000 droplets.

Figure 3.2.5 displays the velocity contour 4" downstream of the spray bars for the 2012 NASA IRT configuration using the calibration nozzle locations. The air jets are 
implemented using the same method that was implemented for the quarter-tunnel simulations expect with the addition of releasing water droplets. For calibrations the nozzles are aligned vertically and horizontally into rows and columns. Arranging the nozzle in this method allows for the development of a transfer map. A transfer map marks the water droplets positions at the test section for every row and column. The column of nozzles immediately next to the inner wall (farthest right column on Fig. 3.2.5) is a column of air only nozzles which never release any water droplets.

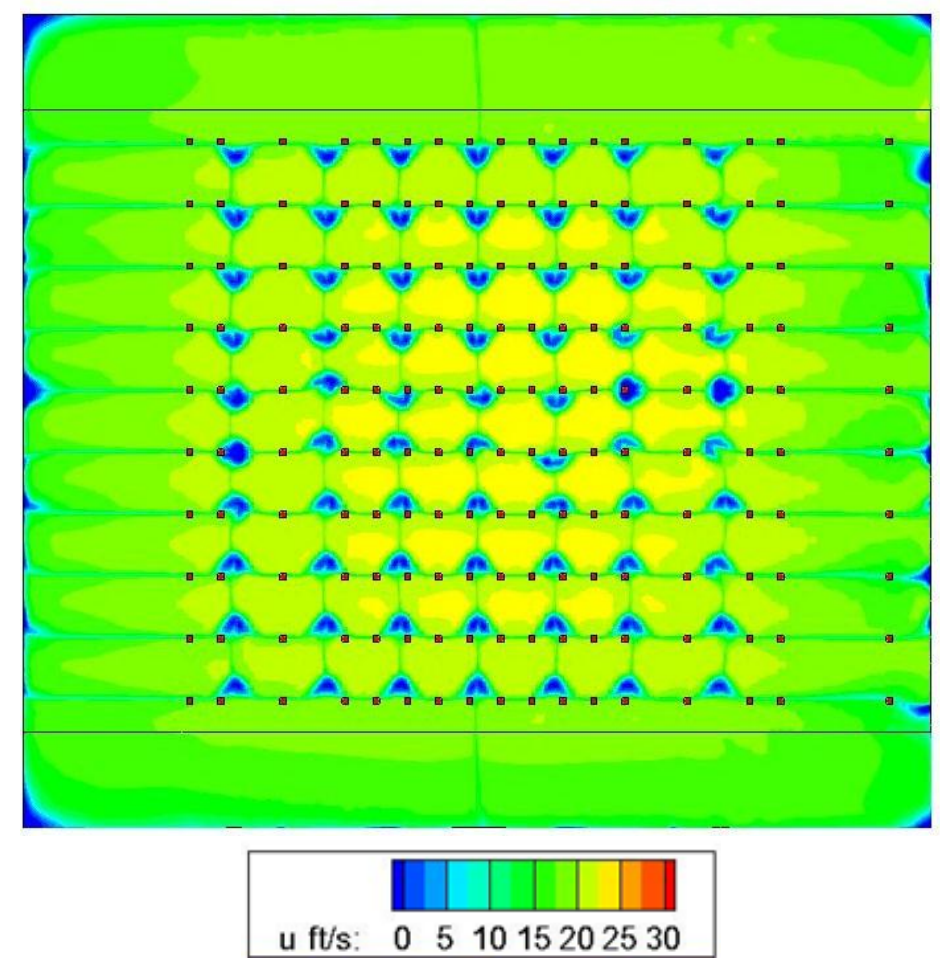

Figure 3.2.5. Velocity contour 4" downstream of spray bars with dark red squares showing active jet locations used to develop the transfer map. These are imaged looking in the upstream direction, i.e. the right side represents the inner wall of the IRT.

Droplets trajectories are calculated from each row of nozzles separately to ensure that the droplet locations at the test section can be easily tracked back to the row from which they were released. Figure 3.2.6 shows the scatter and concentration plot for a single row (the highest row in the IRT). If another row was simulated simultaneously, 
there is a chance that the droplets would start to overlap and increase the difficulty in tracing back every droplet to its source. Figure 3.2.6 shows clearly where the droplets are positioned at the test section for this particular row. A similar process of calculating the droplet trajectories from every column of nozzles individually is also completed and can be seen in Fig. 3.2.7. The column shown in Fig. 3.2.7 is column number 45 (third column from the right in Fig. 3.2.5) and demonstrate the type of results for droplets released from a single column of nozzles.

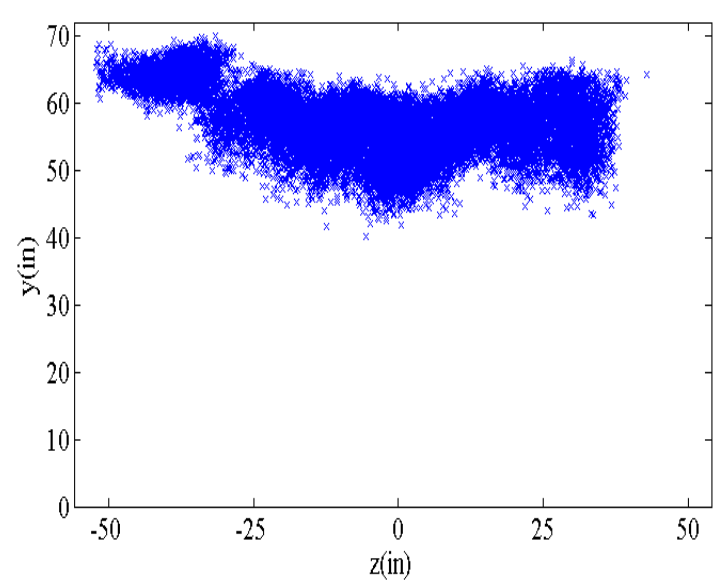

(a)

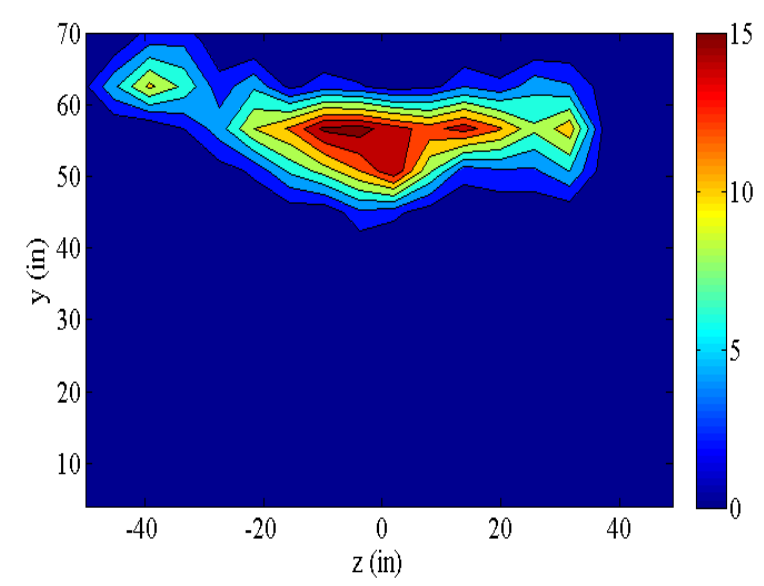

(b)

Figure 3.2.6. Droplet locations at test section from the top bar (bar ten) showed by (a) scatter plot and (b) concentration plot. These are imaged looking in the downstream direction, i.e. the left side represents the inner wall of the IRT.

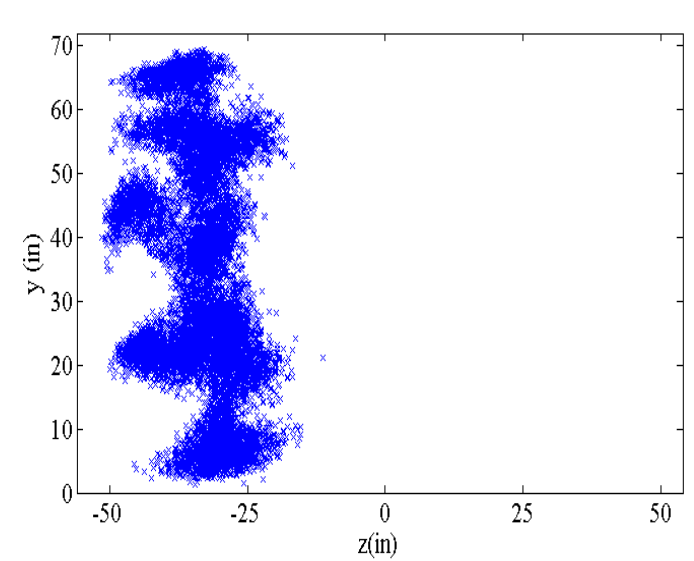

(a)

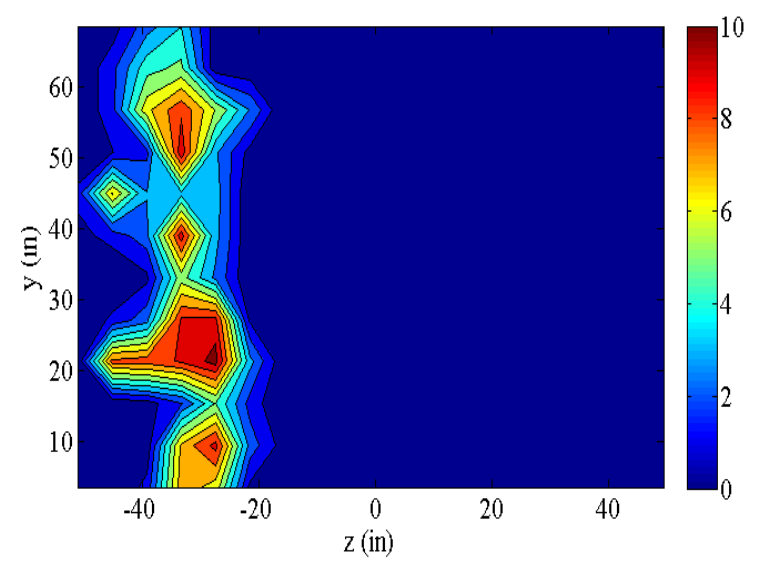

(b)

Figure 3.2.7. Droplet locations at test section from vertical column of nozzles (nozzle column 11) showed by (a) scatter plot and (b) concentration plot. 
Figure 3.2.8 shows the scatter and concentration plot for every nozzle releasing water droplets generated using the data from all the single row and column calculations. The concentration peaks at the center of the test section, then gradually decreases toward the tunnel walls. The high center concentration illustrates the tendency for water droplets to converge towards the center as they travel through the tunnel contraction. The cause for the droplets to converge towards the center of the tunnel is currently unclear. This convergence towards the center is especially true near the spanwise center of the tunnel floor and ceiling which is demonstrated in the concentration and scatter plots by the dip at the center of the ceiling and the bump at the center of the floor. Having the droplets converge towards the center of the tunnel creates a large gradient in LWC at the center of the test section which is highly undesirable because of the tendency of models to be placed at the center of the test section. Further studies are required to understand its cause and solutions to increase LWC uniformity at the center of the test section.

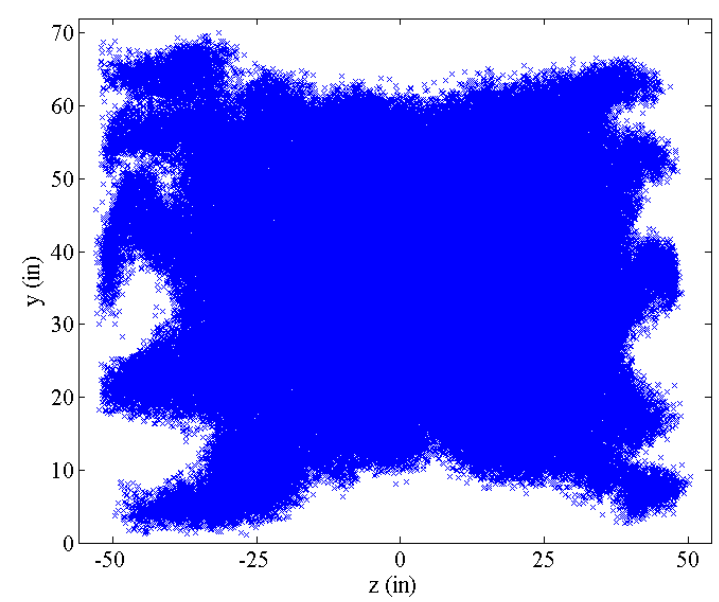

(a)

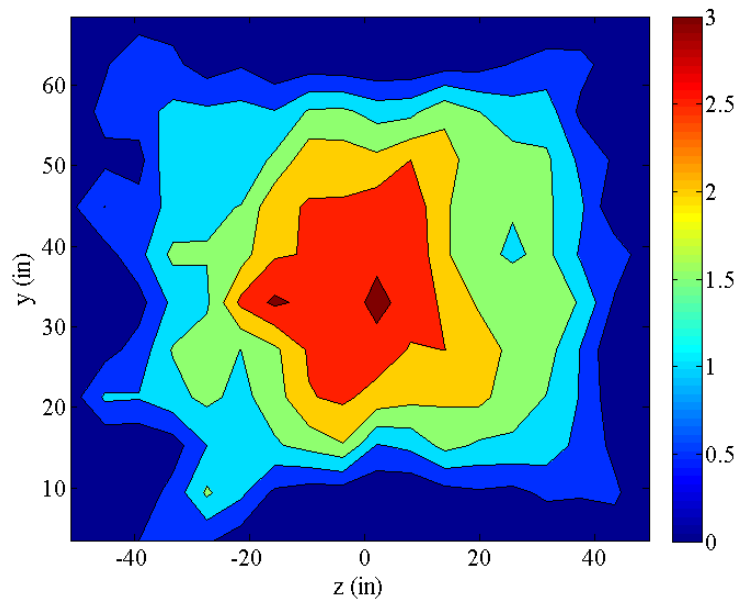

(b)

Figure 3.2.8. Droplet locations at the test section for every nozzle actively releasing droplets as shown by (a) scatter plot and (b) concentration plot. 
Using the solutions generated by the single row and column simulations, e.g. Fig. 3.2.6 and Fig. 3.2.7, a transfer map was created (Fig. 3.2.9). The simulated transfer map is shown in Fig. 3.2.9a and the experimental transfer map is shown in Fig. 3.2.9b. The vertical and horizontal lines map the average water droplets locations at the test section for each row and column of nozzle. The lines for the simulated transfer map are generated using the concentration map with its 5.9" by 5.9" bins and the equation,

$$
Z=\frac{\sum z \alpha}{\alpha_{t o t}}
$$

Equation 3.2.3 sums the concentration multiplied by the horizontal distance for a range of horizontal bins and then divides by the total concentration to give a horizontal location (Z). This equation is used to find the $\mathrm{z}$ location of each section of a vertical line to map the individual columns. The horizontal lines to map the rows are found through a similar process. Calculating the position of the lines this way is equivalent to finding a center of mass of an objected except with concentration values instead of density. The experiments determine the transfer map using the test section grid (Fig. 1.2.8) and visually determine the maximum thickness or centroid location to create the horizontal and vertical lines. 


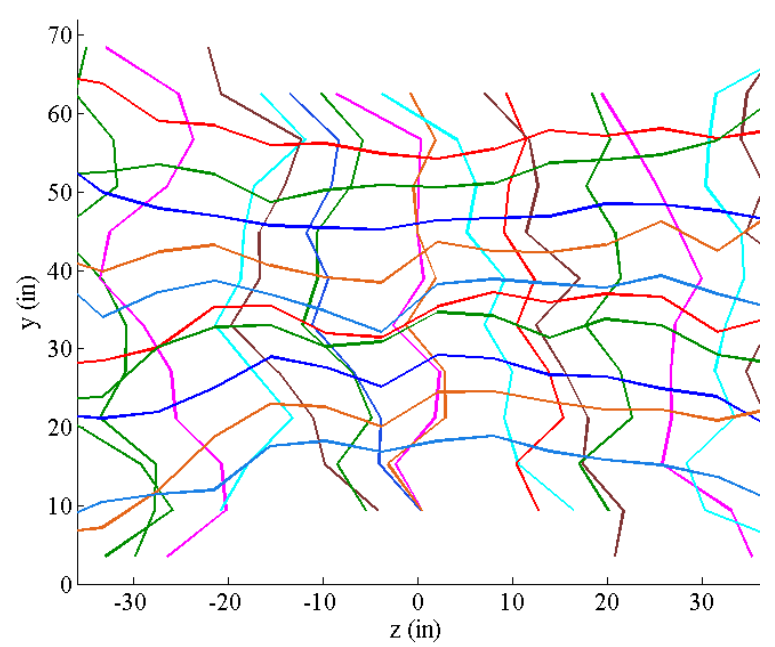

(a)

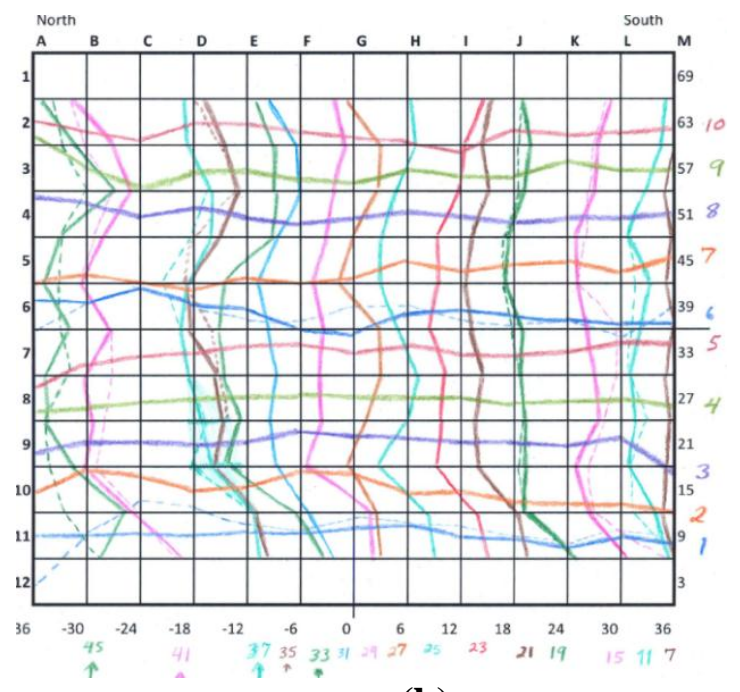

(b)

Figure 3.2.9. Transfer map marking average droplet position at the test section for every row and column that released droplets for (a) simulation and (b) experiments ${ }^{12}$. The plots have an upstream perspective, i.e. the left side represents the inner wall of the IRT and $\mathrm{z}=0$ represents the horizontal center of the tunnel and $\mathrm{y}=0$ represents the floor.

Figure 3.2.9 demonstrates that the simulations are qualitatively reasonable when compared to experiments. The simulations do show more overlap between the columns and more overall fluctuations than the experiments, but the general trends are similar for the two maps. Figure 3.2.9a illustrates the dip and bump in the droplet concentration at the center of the tunnel floor and ceiling respectively by the top and bottom horizontal lines. These lines considerably shift towards the vertical center of the tunnel when they near $\mathrm{z}=0$ (i.e. the spanwise center of the test section). The experiments show a similar trend, but it is not as noticeable as it is for the simulations. The differences in the transfer map indicate that the turbulent diffusion of the droplets is substantially under-predicted. This is attributed to an under-prediction of the RANS model with respect to the turbulence levels downstream of the spray bars. In particular, the RANS is not able to properly capture the spray bar wakes (as described in Part II), for which significant flow separation and vortex generations occurs especially at the intersection of a spray bar and 
vertical strut. In contrast, the spray air jets were modeled adequately with the RANS approach, as evidenced by their good comparison with experiment. A different method, like the hybrid RANS/LES discussed in Part II, would be required to capture the unsteady spray bar wake characteristics and their effect on water droplet distribution. 


\section{Chapter 4. Conculsions}

The air flow within the NASA Glenn Icing Research Tunnel was simulated using a RANS method with a Menter SST model for the flow turbulence. Air flow turbulence within the IRT is required to assist in dispersing the water droplets to create a uniform water cloud, but high turbulence at small length-scaleswithin the test section is unrealistic with respect to actual atmospheric conditions. RANS simulations were utilized to predict the air flow to help understand the turbulence generated from the complex geometries within the NASA Glenn Icing Research Tunnel. A quarter-tunnel simulation of the simple geometry in the 2000 tunnel configuration without struts demonstrated that the RANS method creates a reasonable amount of turbulence when compared to experiments. The simulation also showed that the highest turbulence at the test section is located at the tunnel center and side walls. Additional vertical struts for the 2009 tunnel configuration were incorporated into the quarter-tunnel simulation to analyze their effect on the flow flied. The struts generated additional turbulence throughout the settling chamber, especially behind an intersection of a vertical strut and spray bar. The additional flow disturbances can assist in dispersing the water droplets; however, there is an approximately $75 \%$ increase in velocity fluctuation intensity at the test section which could cause undesired test section conditions. Remarkably the implementation of air jets running at $30 \mathrm{psig}$ does not have a pronounced influence on the turbulence at the test section. The jets do assist in creating a larger spanwise and vertical spread of turbulence which would help create a more uniform droplet concentration.

The new heat exchanger implemented in 2011 for experiments in 2012 is expected to generate more flow turbulence then the 1999 heat exchanger. To simulate the 2012 tunnel configuration, Corner D from the exit of the heat exchanger to the settling 
chamber had to be simulated. A section of the heat exchanger was simulated using a twodimensional model to produce proper inlet conditions for the Corner D simulation. The entire heat exchanger includes six of these sections arranged vertically. The simulations predicted a velocity gradient at the exit of each of these sections with low velocity at the bottom and high velocity at the top. Having these differences in velocity causes a relatively high velocity gradient at a splitter plate where two sections are connected and generates five areas of relatively high turbulent kinetic energy at the exit of the heat exchanger.

The Corner D was simulated using an inlet tabulated from the outlet profile of the two-dimensional heat exchanger simulation. The lack of symmetry required the simulation to incorporate the entire tunnel unlike previous quarter-tunnel simulations. Due to the differences in distances that the air flow has to travel, the inner section of the turn showed much higher levels of turbulent kinetic energy than the outside of Corner D. The larger distances of the outer section of the turn gives more time for the turbulence to dissipate before reaching the spray bars. The flow field at the Corner D outlet was used to calculate the yaw angle for comparison to measurements done experimentally and showed relatively good agreement. Both cases showed high fluctuations in yaw angles due to the installation of the 2012 heat exchanger and the high turbulence flow generated near the splitter plate. New inlet conditions upstream of the spray bars were generated from the Corner D simulation that included much higher gradients in the flow characteristics than the previous quarter-tunnel simulations which assumed a uniform flow before the spray bars. The settling chamber simulation of the 2012 tunnel configuration showed an increase in turbulence with about a 36\% increase in turbulent 
kinetic energy at the test section when compared to the 2009 tunnnel configuration. This turbulence may be higher than desired and modifications may have to be completed to reduce the test section turbulence if test section conditions are inadequate.

Water droplets were released in the RANS simulation of the 2012 NASA IRT configuration to examine the droplet trajectories and locations at the test section. Lagrangian calculations with a discontinuous random walk model were used to calculate the droplet trajectories as they moved from the spray bars to the test section. Scatter and concentration maps were generated at the test section to understand the test section conditions. Releasing droplets from the calibration nozzle locations generated a high concentration of droplets at the test section, which would have to be changed to create more uniform conditions. A transfer map was generated by releasing droplets from a single row and column at a time and marking the droplet locations at the test section. The results were qualitatively correct and indicated a centerline contraction. However, the overall droplet diffusion was under-predicted. This is attributed to the inability of the RANS flow to capture the unsteady spray bar wake effects.

Future droplet simulations should incorporate the 2012 nozzle locations that were developed after the calibrations. These simulations should generate concentration plots that illustrate areas in the test section that could use improved uniformity. New tunnel and spray bar geometries should be investigated to improve the conditions at the test section. Since the RANS model does not factor in any unsteady fluctuations near the water nozzle exit, an LES-based method is recommended to better describe the turbulent diffusion of drops associated with the unsteady fluctuations emanating for the spray bars. 


\section{Part II. Hybrid RANS/LES Simulations of the Spray Bar Region}

\section{Chapter 5. Introduction}

\subsection{Motivation}

Unsteady effects within a flow field such as vortex shedding and instantaneous fluctuations will not be captured properly using a RANS model because RANS simulations converge to a steady state solution which only obtains the mean flow characteristics. Certain jets produced by water nozzles in the NASA Glenn Icing Research Tunnel (IRT) have high intensity flow fluctuations. These unsteady fluctuations cause the jets to rapidly change their spraying direction. Visually the jets that experience these fluctuations appear to be "dancing" when viewed next to jets that do not contain such high intensity fluctuations. The previous RANS computations of the NASA Glenn IRT did not capture these instantaneous fluctuations and their effect on water droplet dispersion, which has been observed experimentally to improve droplet dispersion. Another computational technique will be required to understand the physics behind the "dancing" jets and why only certain IRT nozzles experience this phenomenon.

Large Eddy Simulations (LES) is a computational technique that will resolve the large scale unsteady flow structures instead of modeling them (i.e. RANS). By resolving the large scale flow structures, LES can capture the instantaneous flow variations yielding improved predictions of the "dancing" jet phenomenon. The smallest scales of the flow will still be modeled which reduces the computational demand when compared to a Direct Numerical Simulation (DNS) model that resolves all of the flow scales. A hybrid RANS/LES model was developed because RANS models simulate boundary layers reasonably well from its ability to utilize grids with higher aspect ratios while LES requires finer unilateral grid spacing. This allows for regions near a no-slip surface to be 
modeled by the relatively inexpensive RANS method while the larger flow field to be resolved using a LES method. More information on the process for the hybrid RANS/LES models can be seen in the Methods Chapter below. Unfortunately running a hybrid RANS/LES simulation of the entire tunnel would be too computationally expensive for the resources currently available, so the current study focus on the spray bar region. Simulating this region will assist in understanding the physics behind the unsteady characteristics of a jet flow generated from the IRT water nozzle. An analysis of a video recording the active jets during an IRT experiment was completed before any RANS/LES computations to assist in understanding why certain jets "dance" while others are steadier in their flow direction.

A snapshot of a video observing water nozzles actively releasing air and water droplets during an IRT experiment can be seen in Fig. 5.1.1. The camera is positioned behind the spray bars and directed downstream for a view of the tunnel center near the ceiling. A light on the tunnel ceiling provides enough lighting to monitor 34 different active Mod-1 nozzles and allows for the intensity of the direction fluctuations or "dancing" to be monitored. Fig. 5.1.2 shows three different snapshots of two jet flows initiated from nozzles on the top spray bar. The jet on the left demonstrates high fluctuations in its spraying direction shown by the different spraying angles and the shift in the leftward direction which corresponds to a jet that has high "dancing" intensity. The jet on the right had a nearly constant spray direction and demonstrates little or no "dancing" behavior. 


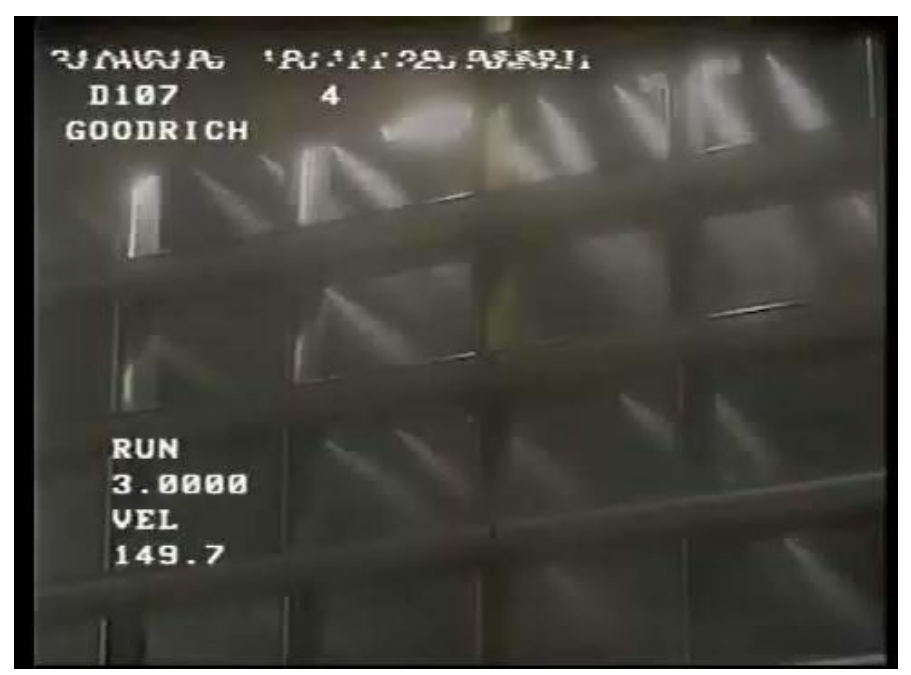

Figure 5.1.1. Snapshot of an IRT video during an experiment which records the "dancing" intensity for multiple Mod-1 nozzles. The video is looking downstream with the left side being the inner wall of the tunnel.
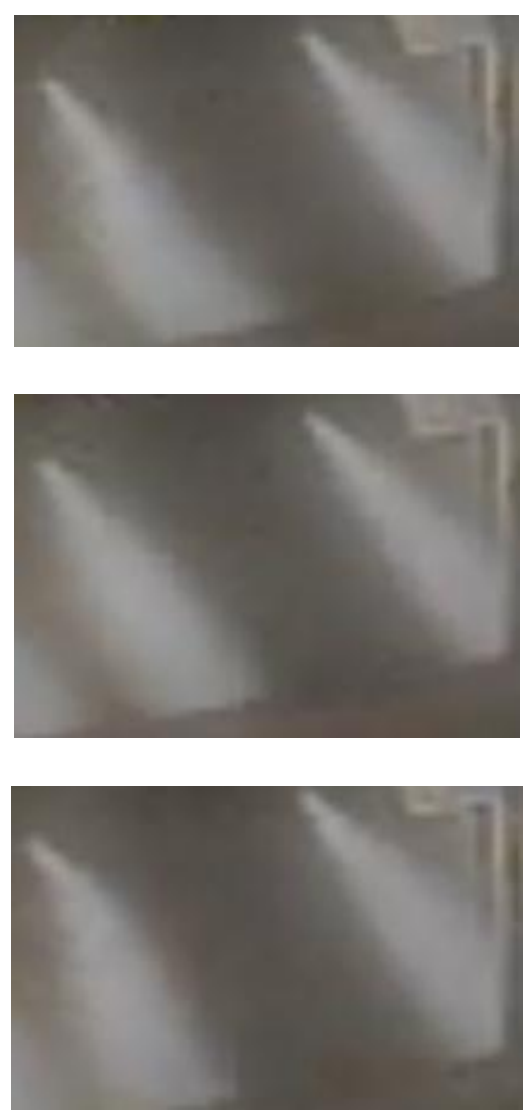

Figure 5.1.2. Time sequence of two nozzles on the top spray bar. The left nozzle had high "dancing" behavior as shown by a significant motion leftward. The right nozzle had low "dancing" behavior with a nearly constant spray direction. 
Figure 5.1.3 is the nozzle map for the Mod-1 nozzles that are active and in view during the recorded video. The map is looking upstream with the right most nozzles position next to the inner wall of the tunnel. The nozzles are colored in terms of the "dancing" intensity demonstrated during the video while nozzles without any color were not able to be sufficiently observed. The "dancing" intensity was determined through qualitative analysis of the recorded video. Highlighted columns at the top of the map illustrate that there is a vertical strut located between these two nozzle locations (e.g. between columns 11-12). Nozzle columns appear to have similar intensities and never contain both a high and low "dancing" nozzle while the rows have a large range of intensities. Every nozzle in a column will have the same distance from a vertical strut and may account for the similarities in "dancing" intensity within a column of nozzles.

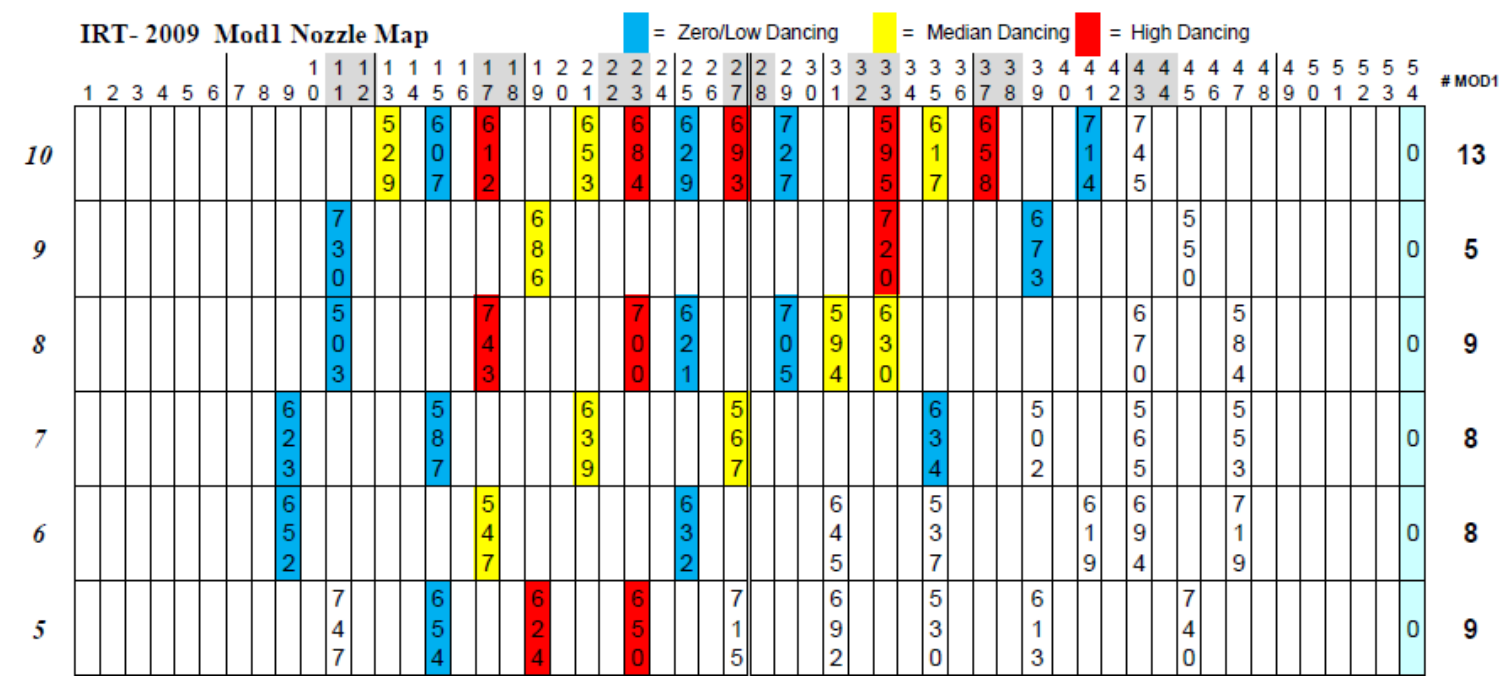

Figure 5.1.3. The "dancing" behavior for the visible Mod-1 Nozzles was measured qualitatively from the video and was categorized into one of three intensities; zero/low, medium and high. The map is looking upstream, i.e. the right side represented the inner wall.

The distance between a nozzle and a vertical strut can either be 3", 9", or 15" because of the number of vertical struts and the 6" distance between nozzles. The number 
of nozzles at three different "dancing" intensities was recorded for the three distances and is shown in a bar graph (Fig. 5.1.4). Nine nozzles with high "dancing" intensity are located 3" away from a vertical strut while only one and zero nozzles have high intensity at 9" and 15 " respectively. The majority of nozzles located at 15 " from a vertical strut have a lower "dancing" intensity; ten nozzles have a zero/low "dancing" rating and the only other nozzle has a medium "dancing" intensity. Figure 5.1.4 demonstrates that there is a relationship between the flow turbulence produced from a vertical strut and the intensity of the unsteady jet fluctuations. Hybrid RANS/LES simulations of this spray bar region will have to demonstrate that they are capturing this increase in "dancing" intensity by comparing a jet with and without a vertical strut and noting the differences in fluctuation intensity.

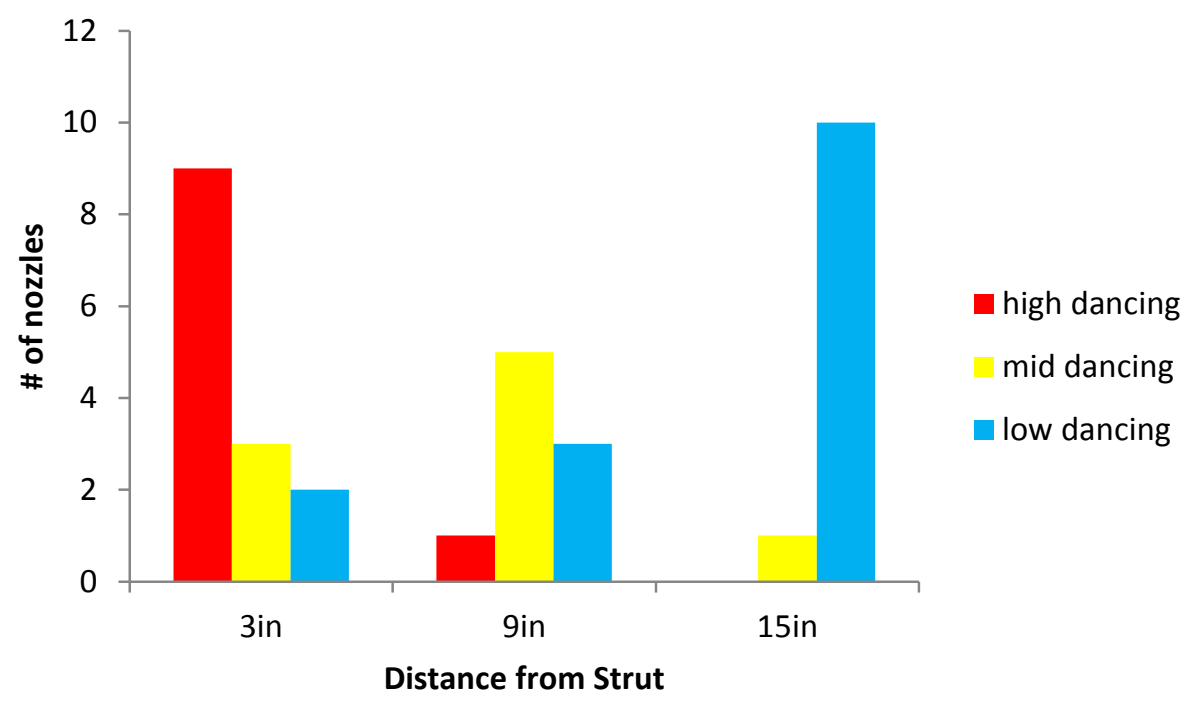

Figure 5.1.4. The number of nozzles at three different "dancing" intensities for the three possible distances from a vertical strut.

The vertical struts that are implemented in the IRT assist in dispersing water droplets because of their ability to increase the "dancing" intensity. Hybrid RANS/LES 
simulations will further explain the physics behind this relationship and why these instantaneous flow fluctuations assist in water droplet dispersion. New spray bar geometries should also be considered and simulated to recreate the "dancing" phenomenon for every water nozzle without the need for vertical struts which create difficulties for spray bar maintenance. An accurate and computationally reasonable LES jet model will be required for any hybrid RANS/LES spray bar simulation with active nozzles even if the nozzles are only releasing air.

Jet flows generate initially small instabilities at the nozzle exit that lead to larger and larger flow structures as they proceed downstream and the turbulence becomes fullydeveloped. As such, fine small-scale resolution is needed at the initial region which requires a small time-step for stability. A small time-step is required because the minimum time-step scales with grid size normalized by the local convection speed. However, the turbulence becomes much larger and the flow is subjected to a reduction in local convection speed as it moves farther downstream (e.g. several jet diameters). The macroscopic unsteadiness is based on these large scales in the flow and generally determines the time integration periods needed to obtain ergodically stationary data from a flow simulation. As such, this pronounced range of time-scales has a profound impact on computational expense. This can be exacerbated for the case when the jet is initially laminar since the initial turbulent structures emanate from small shear layers at the jet edges that are a fraction of the jet diameter.

The problems associated with a single jet are magnified if multiple jets are considered. For our example, the flow in the NASA IRT includes about one hundred Mod-1 nozzles with a diameter of only $3.18 \mathrm{~mm}$, but subsequently interacts in a domain 
that extends nearly $14 \mathrm{~m}$ downstream to the tunnel test section. In this case, simulating the jet flows from the nozzle exits to the test section is computationally impractical with an LES approach. Even modeling a small section of the spray bar region would require multiple jet flows to analyze the unsteady interactions between the jets. These jets will then have to interact with the larger domain as they move towards the tunnel contraction $0.7 \mathrm{~m}(27.5 ")$ from the spray bar trailing edges.

A proper LES jet model needs to make sure that the physics are properly resolved for the entire range of scales especially since the unsteady fluctuations depend on the development of the flow scales as they move downstream. Yet there is a limit to how computationally demanding a simulation can be because of the resources available. Once the jet model has been developed and tested for a single jet flow, it can be implemented into the hybrid RANS/LES simulations of the spray bar region. A hybrid RANS/LES simulation of the spray bar region without a jet model should be developed to make sure of a smooth implementation. Finally once the simulation of the spray bar without an active jet is reasonable the simulation can incorporate vertical struts and active water nozzles to analyze the "dancing" phenomenon and its effect on water droplet dispersion.

\subsection{Previous Studies}

Because of the computational resources required for jet flows, previous computational jet studies tend to focus on either the near-field (e.g. up to ten diameters) or the far-field (e.g. hundreds of diameters), or require extensive resources to simulate both the near- and far-fields. For example, simulations by Muldoon \& Acharya ${ }^{17}$ employed a jet flow domain starting directly at the nozzle exit with a steady velocity

profile but only considered 25 diameters downstream, while simulations by Cai et al. ${ }^{18}$ 
incorporated different jet nozzle geometries with a steady velocity profile but only simulated up to 16 diameters downstream. These studies, focusing exclusively on the near-field region, still require extensive computational resources with fourteen million and four million grid points, respectively. However, these cases employed Direct Numerical Simulation (DNS) which requires higher computational resources than LES. Dinesh et al. ${ }^{19}$ performed LES on a jet flow from the nozzle exit with a steady velocity profile and extending to 60 diameters downstream. These simulations model the nearfield and far-field simultaneously, but, only subsonic jet flows with a mean velocity of 23 $\mathrm{m} / \mathrm{s}$ were considered. A supersonic jet generates smaller time-scales at the nozzle exit, with turbulent structures generated at the shear layer between the supersonic jet flow and free-stream, and would thus require a finer grid than the 2.4 million employed by Dinesh $^{19}$. Using one of these previous jet models would not provide the accuracy in both the near-field and far-field for the spray bar region while still being computationally reasonable.

As discussed in the background in Part I, most of the computational studies of the NASA Glenn IRT are performed with steady state models because of the complex geometry and size of the tunnel. Lee ${ }^{10}$ performed hybrid RANS/LES simulations of a section of the tunnel from the spray bars down to the test section. These simulations included a single spray bar only, spray bar with a vertical strut, and a spray bar with 3-4 active nozzles. However, these simulations did not reproduce the high intensity fluctuations for the jets near a vertical strut, which could have resulted in a combination of factors. First the jet model did not obtain the small scale instabilities from the jet exiting the nozzle but instead created artificial turbulence by issuing a flat velocity profile 
2" downstream of the nozzle called a Plug Jet Profile seen in Fig 5.2.1. Figure 5.2.1 also displays the smooth profile calculated from time-average data obtain from a jet simulation initiated at the nozzle exit. The plug profile created a discontinuity between the jet flow physics and the free stream flow which would generate turbulence not seen from a time-average smooth profile. Since the turbulence was generated artificially, it may not have produced the effects needed to cause the high fluctuations that produce high "dancing" behavior. The second factor that may have caused issues was the fact that the tunnel started to contract right at the spray bar trailing edge when there is actually 27.5 " between the spray bar and the start of the tunnel contraction. This error in geometry could have cause the air flow to start accelerating sooner than reality and prevented any high fluctuations to generate within the jet flow.

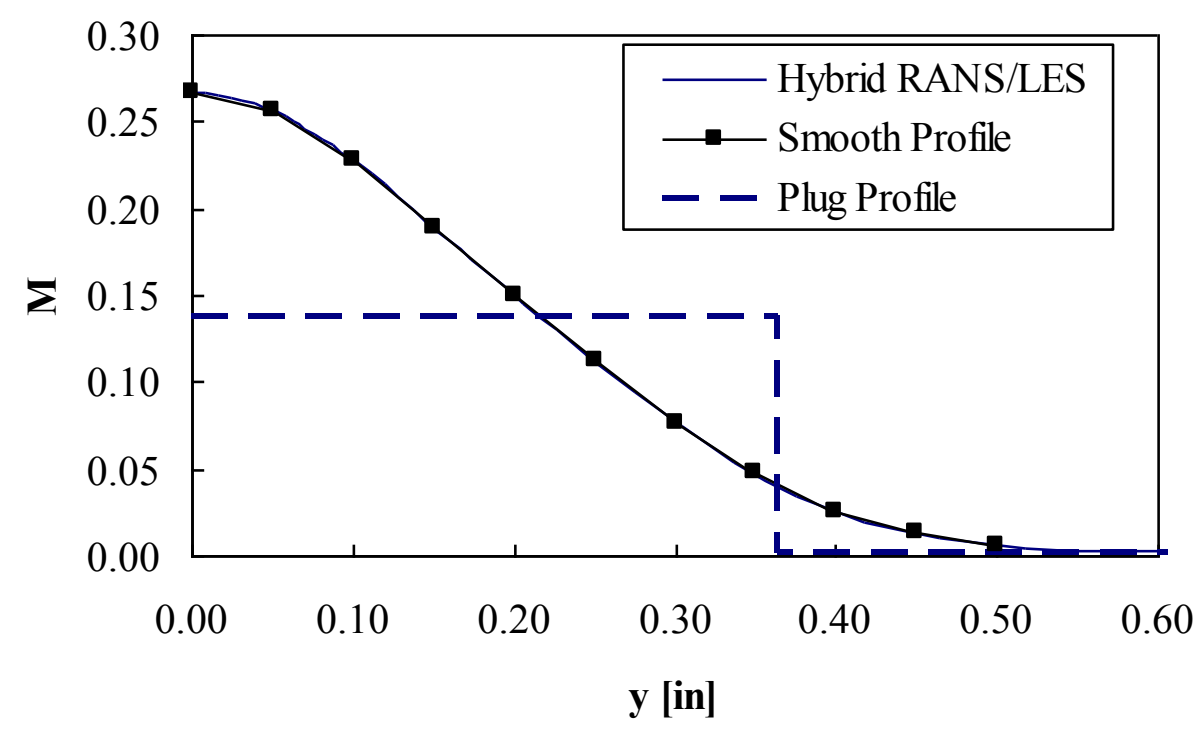

Figure 5.2.1. The time-averaged Mach number profile from Lee ${ }^{10}$ extracted from the full jet simulation $5.08 \mathrm{~cm}$ ( 2 inches) downstream of the nozzle exit and the two profiles used for the jet model in simulations: the smooth profile and the plug profile. 


\subsection{Objectives}

The main objective of the hybrid RANS/LES simulations is to investigate the near spray bar region to understand the physics of the unsteady fluctuations seen in the jet flow released from certain IRT nozzles. The study presented herein developed the techniques and process for future RANS/LES simulations by 1) developing and evaluating a new LES jet model that captures the unsteady flow physics while remaining computationally reasonable and 2) simulating the near spray bar region without any active nozzles or vertical struts. The second objective utilized a hybrid LES/RANS model to simulate the region of the NASA Glenn IRT near a single spray bar. The simulation will not incorporate any active jets or vertical struts but remained simple to provide a base simulation to add these components into. The flow structures behind the single spray bar were analyzed and compared to the RANS model to examine its necessity and the improvement in modeling certain flow structures. The simulations will only incorporate the tunnel up to the tunnel contraction instead of simulating all the way to the test section as seen in Lee ${ }^{10}$ which frees computational resources to allow for a finer grid near the spray bar region.

The first objective addresses a single jet flow without any interaction with a spray bar or strut to allow for the development of an accurate jet model. Since there are no boundary layers or no-slip surfaces the hybrid RANS/LES model will convert to a pure LES model. To address the high CPU cost associated with the very large time-scale range produced by a jet flow, a new multi-scale method was developed that separates the simulation close to the nozzle exit (near-field) and the simulation farther downstream (far-field). Separating the two fields decreases grid resolution and time-step for the farfield to help reduce time per iteration and total number of iterations. The key is to 
establish an unsteady interface condition which couples these two fields in a computationally-efficient and numerically-accurate manner. This study utilizes a Recorded Interface Boundary Condition (RIBC), whereby the instantaneous data exiting the near-field is recorded and replayed in a cyclical fashion for the far-field. This multiscale approach can dramatically relieve the computational demand when compared to the conventional full-field approach, whereby both fields are simulated simultaneously. Note that the present approach is similar to the rescaling-reintroducing method of Urbin and Knight $^{20}$ whereby a recycling region upstream of the domain of interest is established. The turbulent boundary layer structures at the outlet of this domain are rescaled according to inner and outer scaling, and the result is fed back into the upstream portion of the recycling domain. After several flow sweeps through this recycling domain, a fully-developed turbulent boundary layer emanates. The computational domain and cost for this approach are far lower than explicitly simulating the boundary layer development including laminar to turbulent transition. This approach works particularly well for pipe and attached boundary flows. It has also been employed for LES jet studies (Yuan et $a l^{21}$ ) to develop the flow within the pipe leading to the jet nozzle. However, once the flow leaves the nozzle its turbulent length- and time-scales will begin to increase while the mean velocity will decrease. Due to this, recycling the downstream physics back to the nozzle exit would not generate the appropriate flow characteristics. While the recycling method would generate incorrect physics if applied to the development of a jet downstream of the nozzle, the flow reintroduction concept can still be utilized. The jet flow can be simulated within the near-field and reintroduced in the far-field using a RIBC. This method will reasonably generate the mean and turbulent velocities while only 
simulating the near-field once instead of repeatedly recycling the near-field. For this first objective, the methodology for both the full-field and multi-scale approaches will be discussed, followed by investigation and assessment of two variants of the Recorded Interface Boundary Condition. Completing both of these objectives will establish a foundation for future hybrid RANS/LES simulations that will investigate the "dancing" phenomenon. 


\section{Chapter 6. Methods}

\subsection{Hybrid RANS/LES Numerical Method}

Large Eddy Simulations (LES) incorporate the same governing equations for fluid motion discuss in Part I. However, the variables are not separated into mean and fluctuating components (seen in RANS models) which allow for instantaneous fluctuations within the flow field to be resolved. To capture every flow length scale, which occurs in a Direct Numerical Simulation (DNS), would require the computational grid to have extremely fine spacing. To ease the computational cost of the simulations the governing equations are put through a spatial filter seen below.

$$
\bar{q}\left(x_{i}\right)=\int G\left(x_{i}, x_{i}^{\prime}\right) q\left(x_{i}^{\prime}\right) d x_{i}
$$

Where $G\left(x_{i}, x_{i}^{\prime}\right)$ is a filter function which varies from case to case but always involves a length scale $(\Delta)$ that determines when a flow scale is small enough to be modeled. The filtered momentum equation for the case of incompressible flow becomes a form that is similar to a RANS model i.e.

$$
\frac{\partial\left(\rho \bar{u}_{i}\right)}{\partial t}+\frac{\partial\left(\rho \overline{u_{i} u_{j}}\right)}{\partial x_{i}}=-\frac{\partial \bar{p}}{\partial x_{i}}+\frac{\partial}{\partial x_{j}}\left[\mu\left(\frac{\partial \bar{u}_{i}}{\partial x_{j}}+\frac{\partial \overline{u_{j}}}{\partial x_{i}}\right)\right]
$$

The term $\overline{u_{i} u_{j}}$ is not easily computed because of the condition

$$
\overline{u_{i} u_{j}} \neq \overline{u_{i}} \overline{u_{j}}
$$

which leads to a modeling approximation for the difference between the two terms:

$$
\tau_{i j}^{s}=-\rho\left(\overline{u_{i} u_{j}}-\overline{u_{i}} \overline{u_{j}}\right)
$$

$\tau^{s}{ }_{i j}$ is called the sub-grid scale Reynolds stress and is calculated through the sub-grid $\operatorname{model}^{22}$. 
The purpose of the hybrid RANS/LES methods is to reduce the high computational cost that LES simulations require when the turbulent length scale starts to dramatically reduce. This is a common issue with LES simulations incorporating boundary layers formed from no-slip surfaces. These surfaces require very fine resolution meshes to properly resolve these length scales as they develop instead of relying heavily on the sub-grid model. The hybrid RANS/LES method allocates these regions to a RANS based solver which requires much lower computational demand discussed in Part I.

The Nichols-Nelson hybrid RANS/LES ${ }^{23}$ approach based on the Menter SST k- $\omega$ turbulence model was chosen to model the turbulence. The turbulent kinetic energy predicted with this method is the sum of the resolved turbulent kinetic energy and the modeled sub-grid turbulent kinetic energy:

$$
k=k_{r e s}+k_{\text {sub }}
$$

The resolved turbulent kinetic energy is defined as half of the sum of the mean velocity fluctuations, i.e.

$$
k_{r e s}=\frac{1}{2}\left(u_{x, r m s}^{2}+u_{y, r m s}^{2}+u_{z, r m s}^{2}\right)
$$

The resolved turbulence will dominate in the LES regions of the flow which can be seen in the single jet section (chapter 7). The sub-grid turbulent kinetic energy is related to the RANS turbulent length scale given by

$$
\Lambda_{\text {RANS }}=\max \left(6.0 \sqrt{v_{R A N S} / \Omega_{\text {RANS }}}, k_{R A N S}^{3 / 2} / \varepsilon_{R A N S}\right)
$$

In this expression, $v_{R A N S}$ is the eddy viscosity, $\varepsilon_{R A N S}$ is the turbulent dissipation, and $\Omega_{R A N S}$ is the local resolved flow vorticity. The sub-grid kinetic energy is calculated based on the RANS component 


$$
k_{\text {sub }}=k_{\text {RANS }} \xi
$$

and the clipping function $\xi$ is defined as

$$
\xi=\frac{1}{2}\left\{1-\tanh \left[\frac{\pi\left(\Lambda_{R A N S}\right)^{4 / 3}-\pi(2 \Delta)^{4 / 3}}{\left(\Lambda_{R A N S}\right)^{4 / 3}-(2 \Delta)^{4 / 3}}\right]\right\}
$$

where $\Delta$ is the grid length scale determined by the filtering function. The eddy viscosity for this hybrid scheme can be calculated as

$$
v_{h y b}=v_{R A N S} \xi+(1-\xi) v_{\Delta}
$$

where $v_{\text {RANS }}$ is calculated from the RANS-SST model and $v_{\Delta}$ is the sub-grid eddy viscosity given by

$$
v_{\Delta}=\min \left(0.0854 \Delta \sqrt{k_{R A N S}}, v_{R A N S}\right)
$$

The 0.0854 is derived from the one-equation $\mathrm{k}$-model and determines the transition from RANS to LES. Equation 6.1.11 insures that there will be a smooth transition when the simulation transitions from RANS to LES or vice versa.

The current hybrid RANS/LES simulations utilize a Roe fifth order upwindbiased scheme for the spatial discretization. This scheme is an explicit scheme that focuses on calculating the governing equations using values obtained from the direction of mean flow velocity (i.e. upwind). Temporal discretization was handled by a full second order implicit scheme. A constant time-step had to be specified for the entire domain because the hybrid model is a time-dependent scheme. A simple cylinder case was simulated to ensure that the hybrid RANS/LES is predicting reasonable flow physics.

The hybrid RANS/LES method was employed by the WIND-US 2.0 package developed by the National Program of Applications-Orientated Research in CFD 
(NPAORC) Alliance. An example of the input file required by WIND can be seen in Appendix B. Grid generation and data processing were performed by Gridgen and TECPLOT respectively, which was done for the RANS tunnel simulations.

\subsection{Cylinder Wake Validation}

A simple simulation of a cylinder within a flow with a Reynolds number of 8000 was completed to validate the accuracy of this hybrid RANS/LES scheme. A cylinder flow is a case that has been analyzed and investigated multiple times which gives an abundant amount of data for comparison. The frequency of the vortices that were generated within the cylinder wake is the main focus for validation. Vortex shredding is a highly unsteady phenomenon that requires accurate calculations of the unsteady fluctuations. Comparing these simulated fluctuations behind the cylinder to experiments and DNS calculations will determine the fidelity of the hybrid RANS/LES model.

The cylinder simulations utilized a three dimensional structured O-grid around a cylinder with a 2.4" diameter. The computational grid, seen in Fig. 6.2.1, is broken into three different zones with dimensions $150 \times 37 \times 42,74 \times 37 \times 42$, and $59 \times 74 \times 42$. The cylinder boundary is a no-slip wall while the outer edge of the O-grid was set to freestream conditions of 0.1 Mach number in the streamwise direction. The simulation ran for 5000 iterations with a time step of $6 \times 10^{-5}$ seconds after the solution was determined to be converged (i.e. without any errors from initial conditions). 


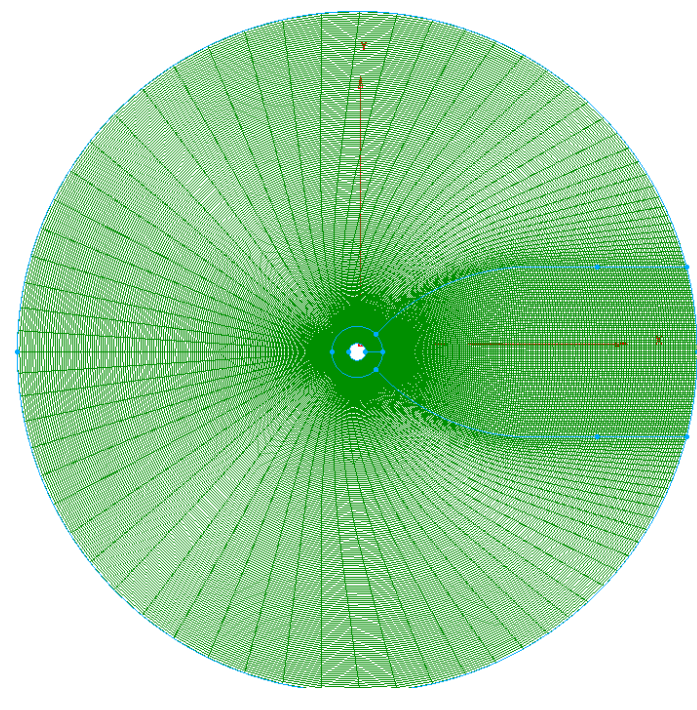

(a)

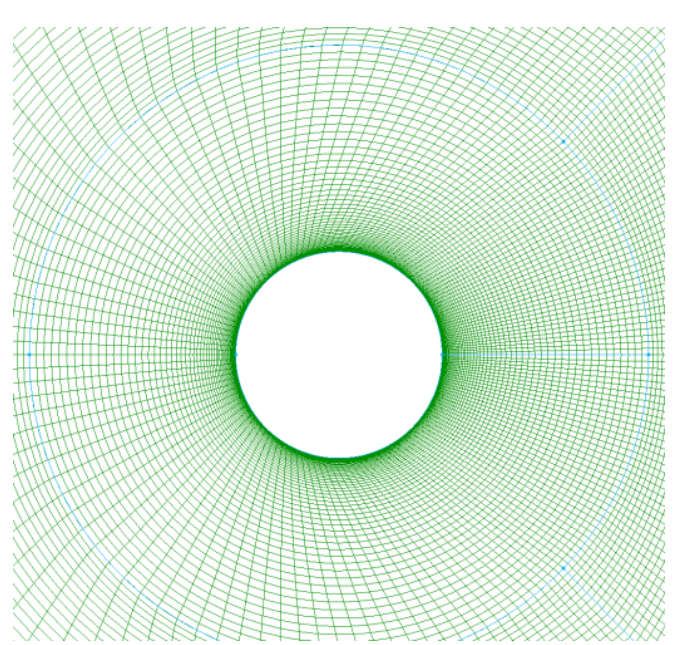

(b)

Figure 6.2.1. Computational grid from Lee $^{10}$ used for the cylinder wake validation: (a) entire computational grid and (b) close-up view of grid around cylinder.

Velocity in each of the directions at every time step was recorded 2.4 inches (one diameter) downstream from the trailing edge of the cylinder. These velocities underwent a Fast Fourier Transform (FFT) which solves the discrete Fourier Transform (DFT) shown below ${ }^{24}$. Figure $6.2 .2 \mathrm{a}$ is the power spectrum density calculated from DNS cylinder simulations performed by Dong ${ }^{25}$. Figure $6.2 .2 \mathrm{~b}$ graphs the power spectrum density for the current cylinder simulations calculated using a FFT of the streamwise and crossflow velocities. The power spectrum density for both the current study and simulations by Dong demonstrate similar treads in decay for larger frequencies. Where the power spectrum density peaks illustrates the frequency of the instabilities within the cylinder wake. The current simulation shows a peak around a Strouhal number of .233 as illustrated clearly in Fig. 6.2.2c. The Strouhal number is calculated by,

$$
S t=\frac{f D}{u}
$$


where $f$ is the frequency, $D$ is the diameter of the cylinder and $u$ is the free stream velocity. The Strouhal number provides a dimensionless number for frequency to compare between different cases. Figure 6.2.3 compares the Strouhal number for multiple cylinder experiments and simulations for a range of Reynolds numbers. The majority of the cases have Strouhal numbers in the range between 0.15 and 0.3 which gives confidence for the hybrid RANS/LES with a prediction of 0.23 and validates its use for simulating unsteady phenomenon. 


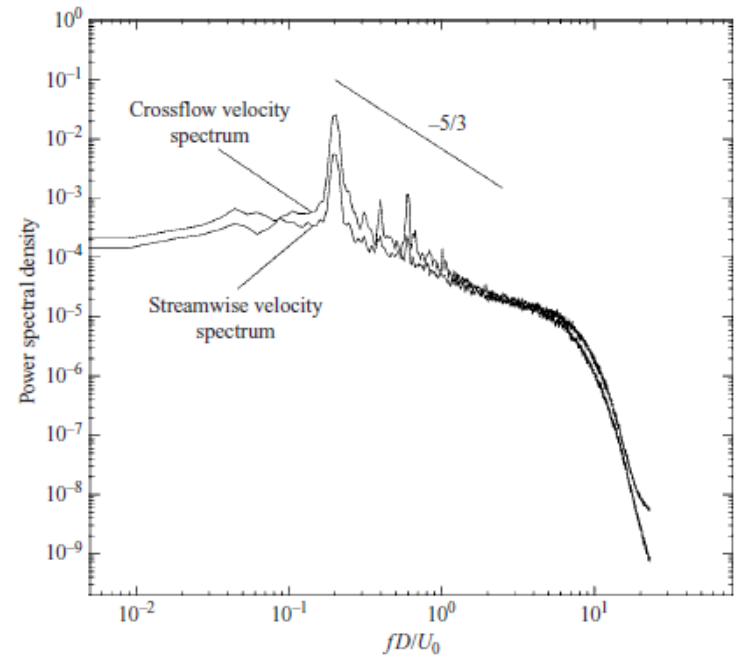

(a)

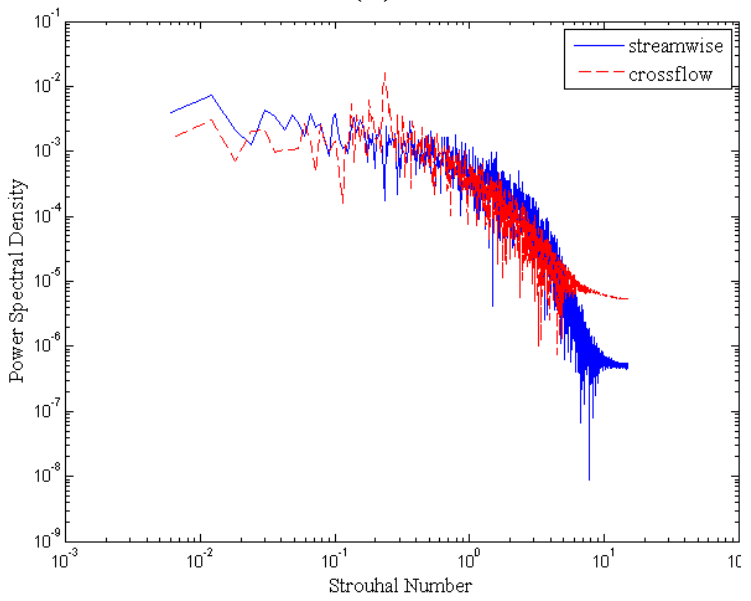

(b)

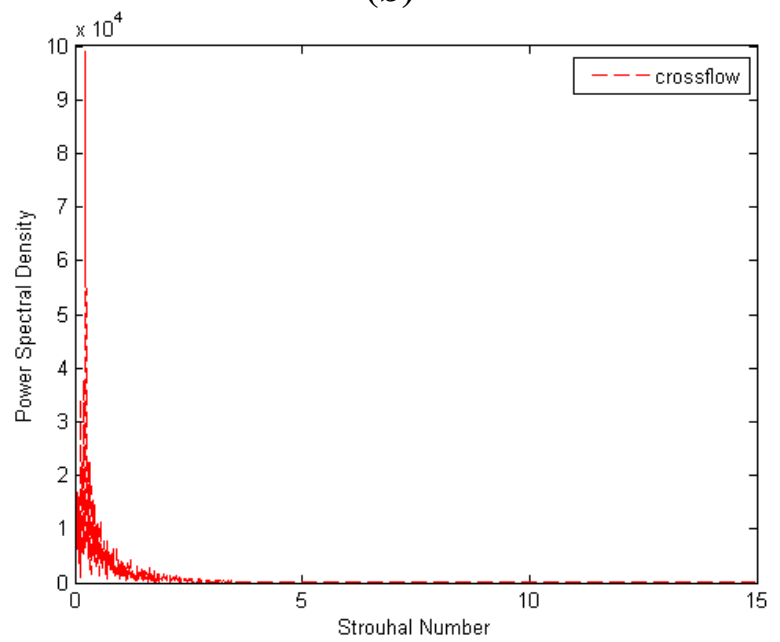

(c)

Figure 6.2.2: Spectral density of the wake behind a cylinder from (a) $\operatorname{Dong}^{25}$, (b) present predictions. (c) The present predictions without a log-log plot to illustrate the Strouhal number location of the peak density. 


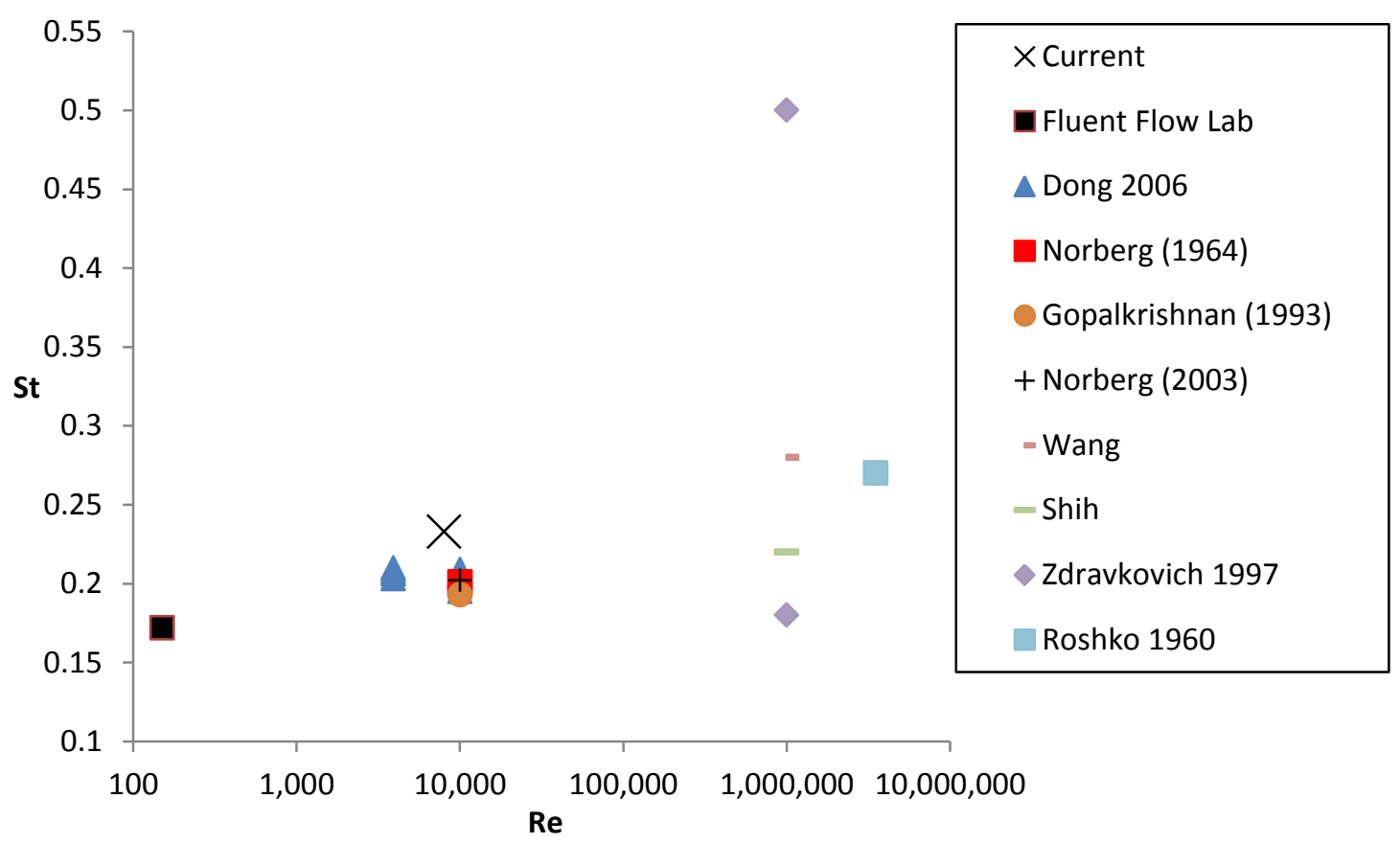

Figure 6.2.3. Comparison of current Strouhal number with previous cylinder studies. 


\section{Chapter 7. New Coupling Method of Near-field and Far-Field for Jet Flows}

\subsection{Single Jet Computational Domain}

Figure 2.1.2 shows a schematic of the Mod-1 nozzle used within the NASA Icing Research Tunnel and is the base nozzle model for the LES jet simulations for developing the jet model conducted herein ${ }^{13}$. It has a diameter of $3.18 \mathrm{~mm}$. The current study focuses on the air flow and assumes that no water is being released from the nozzle. For the fullfield and near-field jet simulations, the nozzle gas pressure was set at 20 psig (34.7 psi) yielding choked conditions based on the experiments of Bulzan ${ }^{9}$. As described by Lee ${ }^{10}$, the actual jet narrows somewhat before exiting the flush plate. To account for this, the computational exit diameter is set at $2.28 \mathrm{~mm}$ consistent with choked conditions (a Mach number of 1), an exit pressure of $34.7 \mathrm{psi}$, and the experimental gas flow rate at $2.25 \mathrm{~g} / \mathrm{s}$. This yields an exit velocity of $315 \mathrm{~m} / \mathrm{s}$. At the inflow plane just outside the jet (i.e. at $\mathrm{r}>1.14 \mathrm{~mm}$ ) and to the sidewall, the flow is set at standard atmospheric pressure and temperature with a weak axial co-flow of $0.035 \mathrm{~m} / \mathrm{s}$ (corresponding to a Mach number of 0.001) to ensure computational stability and eliminate any reverse flow conditions. The outflow boundary condition opposite of the nozzle is an outlet set to atmospheric pressure. The center of the grid is set as a singular axis boundary condition which interpolates its values from the adjacent grid points encircling the axis.

The computational grid follows that of Lee ${ }^{10}$ which was found to give gridindependent predictions of the mean and turbulent kinetic energy as far as $20 \mathrm{~cm}$ downstream. Figure 7.1.1 shows isometric and planar views of the computational cylinder grid for the full-field simulation with 850,000 cells. The domain is $51 \mathrm{~cm}$ long (starting from the jet exit) and has a radius of $15 \mathrm{~cm}$. The grid was set to be finest near the jet exit and along the shear layer. The time-step $(\Delta t)$ for the full simulation is based 
off the grid spacing near the nozzle exit and is set at $4 \times 10^{-7}$ seconds, which was found to be suitable in terms of both numerical stability and time-step independence of the resulting flow solution ${ }^{10}$.

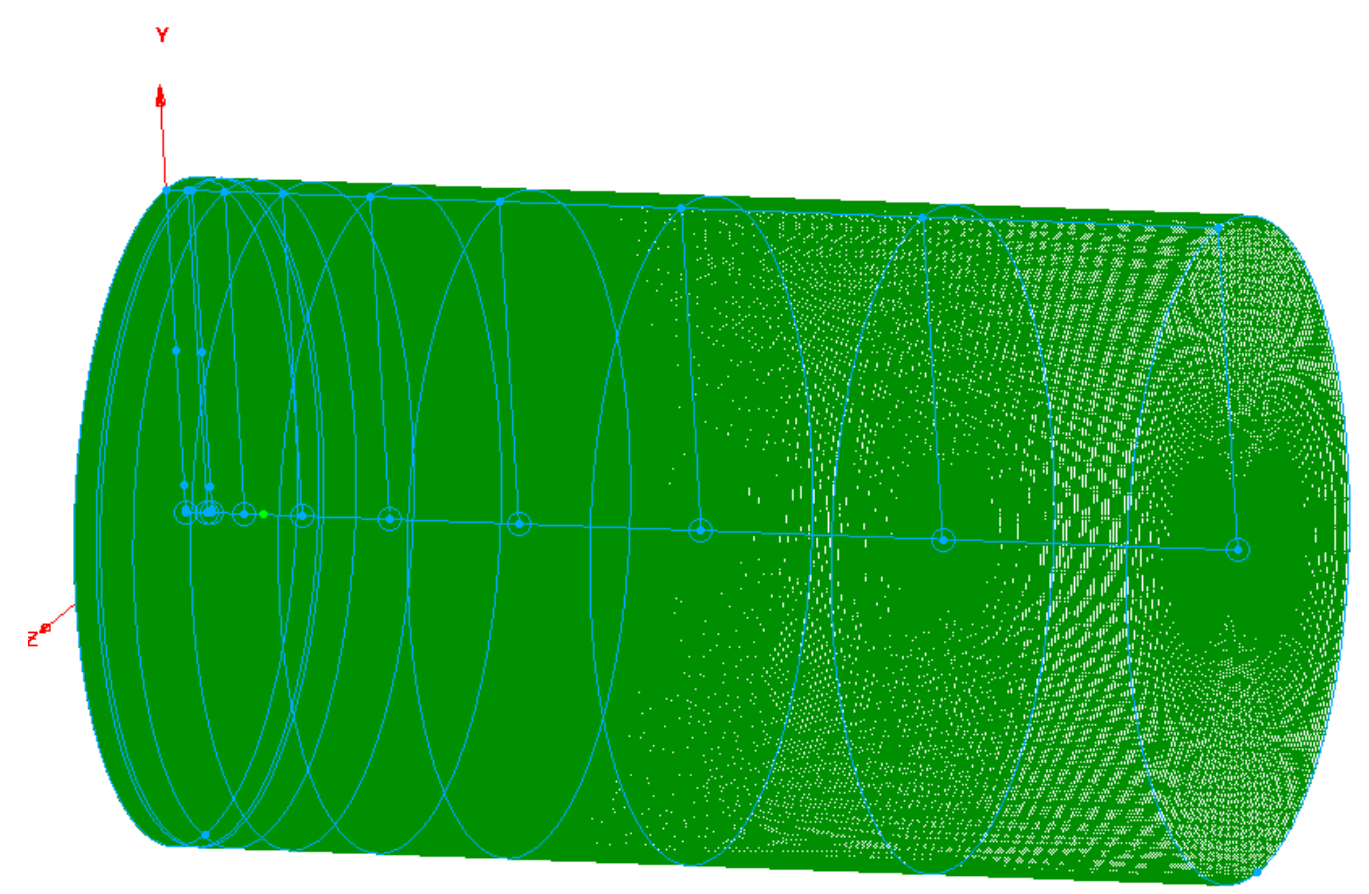

(a)

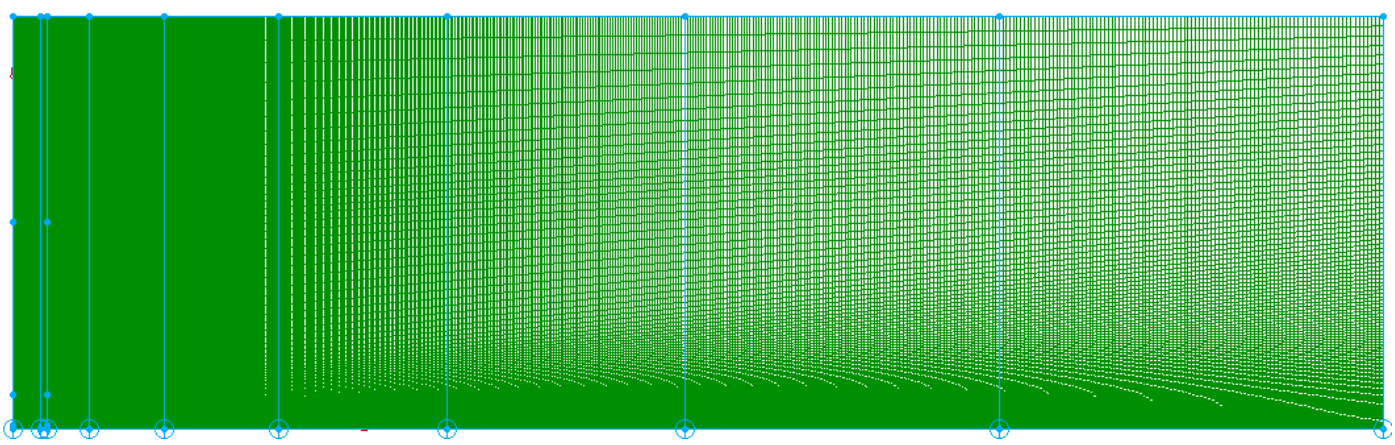

(b)

Figure 7.1.1. Computational domain of full-field jet simulation (a) isometric view of 3D mesh (b) axisymmetric view of the mesh showing radial and streamwise resolution.

To investigate the RIBC, both near-field and far-field grids were also constructed. A schematic showing all three computational domains is shown in Fig. 7.1.2 where it can 
be seen that the full-field domain is divided into near-field and far-field domains separated by a coupling interface which is $1.5 "(3.8 \mathrm{~cm})$ downstream of the jet exit. Table 7.1.1 displays the number of grid points $(\mathrm{N})$, the time-step, the integration period $(\mathrm{T})$ required for statistical converge of the time-averaged kinetic energy, the corresponding number of time-steps (I), and the CPU time normalized by that for the full-field simulation, where the CPU time is assumed to be proportional to $\mathrm{N}^{*}$ time-step. Having a lower combined CPU time for the near- and far-field simulations when compared to the full-field simulation is due to the larger time-step that can be utilized for the far-field domain.

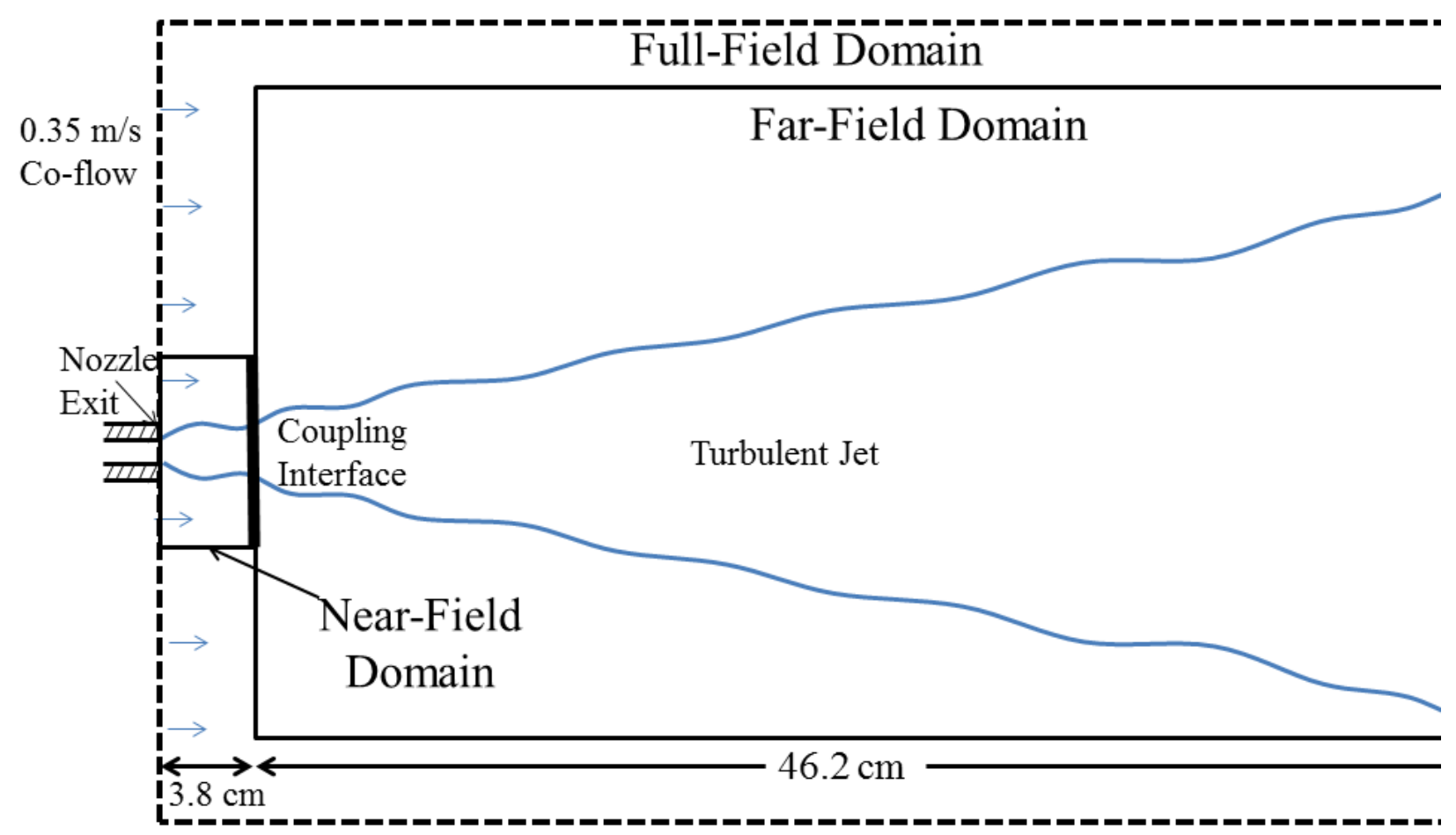

Figure 7.1.2 . Schematic of near, far, and full-field domains. 


\begin{tabular}{|c|c|c|c|c|c|}
\hline Domain & $\begin{array}{c}\text { Number of } \\
\text { Grid } \\
\text { Points }\end{array}$ & $\Delta \mathbf{t}(\mathbf{s e c})$ & $\begin{array}{c}\mathbf{I} \\
\text { Number of } \\
\text { Iterations }\end{array}$ & $\begin{array}{c}\text { T (sec) } \\
\text { Period of } \\
\text { Integration }\end{array}$ & $\begin{array}{c}\text { CPU Time } \\
\text { Normalized } \\
\text { by Full-Field }\end{array}$ \\
\hline Full-Field & $8.5 \times 10^{5}$ & $4 \times 10^{-8}$ & $2 \times 10^{6}$ & $8 \times 10^{-2}$ & 1.000 \\
\hline Near-Field & $2.6 \times 10^{5}$ & $4 \times 10^{-8}$ & $1 \times 10^{5}$ & $4 \times 10^{-3}$ & 0.015 \\
\hline Far-Field & $6.4 \times 10^{5}$ & $4 \times 10^{-7}$ & $2 \times 10^{5}$ & $8 \times 10^{-2}$ & 0.075 \\
\hline
\end{tabular}

Table 7.1.1. Information of the computational demand for each LES jet simulation.

\subsection{Jet Interface Coupling}

The interface between the near and far-field seen in Fig. 7.1.2 is at an axial slice $3.8 \mathrm{~cm}$ (1.5 inches) downstream. At this distance downstream the peak Mach number (M) is 0.7 , and so this region is downstream of the transonic diamond shock pattern generated at the choked nozzle. This means flow is fully subsonic, the pressure field is nearly uniform and equal to the ambient pressure, and the convective velocity is always found to be positive (no reverse flow), one may posit that the turbulent flow structures and the mean flow may be assumed to convect downstream with little upstream influence. This is the primary assumption of the interface coupling schemes investigated in this study and effectively assumes that the flow field characteristics are parabolic, i.e., is similar to the classical Parabolized Navier-Stokes (PNS) approach ${ }^{5}$. This parabolic flow assumption is not reasonable in flows with significant flow curvature, reverse flow regions, or no-slip surfaces (where upstream influence can be strong in the near-wall no-slip portion). Furthermore, this assumption is unreasonable with respect to resolving pressure 
fluctuations, which clearly require upstream communication. Its use with LES has not been previously investigated to the authors' knowledge.

Given the above strong assumption (that the flow can be considered approximately parabolic at the interface plane), one may consider two classes of coupling interface boundary condition: steady radially-symmetric and unsteady asymmetric. For a steady boundary condition, the time-averaged properties from the near-field LES flow at the coupling interface can be obtained as a function of radius and then simply specified as steady input conditions for the far-field domain much like the smooth profile by Lee ${ }^{10}$ seen in Fig. 4.2.1. However, all the near-field unsteadiness is lost and the result is a jet inconsistent with the full-field results discussed in the results section (7.3). Therefore this case was not pursued for the current study.

For an unsteady interface coupling scheme, the flow field quantities along the interface plane are recorded as a function of time starting from a fully-developed flow within the near-field LES simulation. The spatial and temporal recording of the flow variables (mass, momentum, energy, and turbulence variables) is within a radius of .55" $(1.4 \mathrm{~cm})$ and over a period of $4 \times 10^{-3}$ seconds (per Table 7.1.1). This time period corresponds to approximately 40 sweeps of the flow in the near-field domain from the jet exit to the interface plane. The recording radius of .55 " was sufficiently far from the jet centerline such that the velocities were less than $1.8 \mathrm{~m} / \mathrm{s}$ and could be approximated as equal to the steady co-flow velocity.

Since this data is used at the inflow of the far-field region, the temporal resolution required is that for the far-field time-step. For simplicity, this time-step was set to be ten times that of the near-field and was found to provide predictions that were time-step 
independent. As such, the instantaneous data from the near-field was recorded every 10 iterations (for 10,000 iterations) so that it would be consistent with the far-field simulation. However, since the far-field involved much slower time-scales, it required overall integration periods that were twenty times longer than those needed for the nearfield (i.e. $\mathrm{T}_{\mathrm{far}}=20 \mathrm{~T}_{\text {near }}$ ). One option would be to record the near-field for a commensurate length of time. However, much less CPU is required if the near-field recording could be replayed twenty times during the far-field integration. This is the approach employed herein, but it requires special treatment at the end of each playback where the near-field recording at the interface plane is a stored input during the computation.

Figure 7.2.1 graphically shows the two variants employed to handle this issue: one is to use a forward-only profile and the other is to use a combination of forward and backwards profiles. For the "forward-only" profile, the near-field recording is played back over a period of $\mathrm{T}_{\text {near }}$. Once the period is reached, the recording is simply replayed from the initial time, and the process is repeated throughout the far-field integration period. However, this process creates a discontinuous temporal jump in the flow variables. While this discontinuity is only applied to the turbulent fluctuations, which are weak compared to the mean flow, it introduces a local error (and can lead to numerical instabilities). Therefore, a second choice is to play the recorded history at the interface plane backwards in time, after which the forward-profile is replayed, and so on. This reverse order is termed the "forward and backward profile" and prevents the discontinuous jump at the end of the recording. This variant may be reasonable for ergodically steady flows because the Navier-Stokes equations are equally valid whether integrated forwards or backwards in time. While ensuring a smoother transition, this 
forward and backward profile does yields a discontinuity in the time-derivative of the flow quantities, e.g. in the acceleration. Both profiles were investigated in the present study for the jet flow and compared to both experiments by Bulzan ${ }^{9}$ and full-field simulations computed by Lee ${ }^{10}$ as will be discussed.

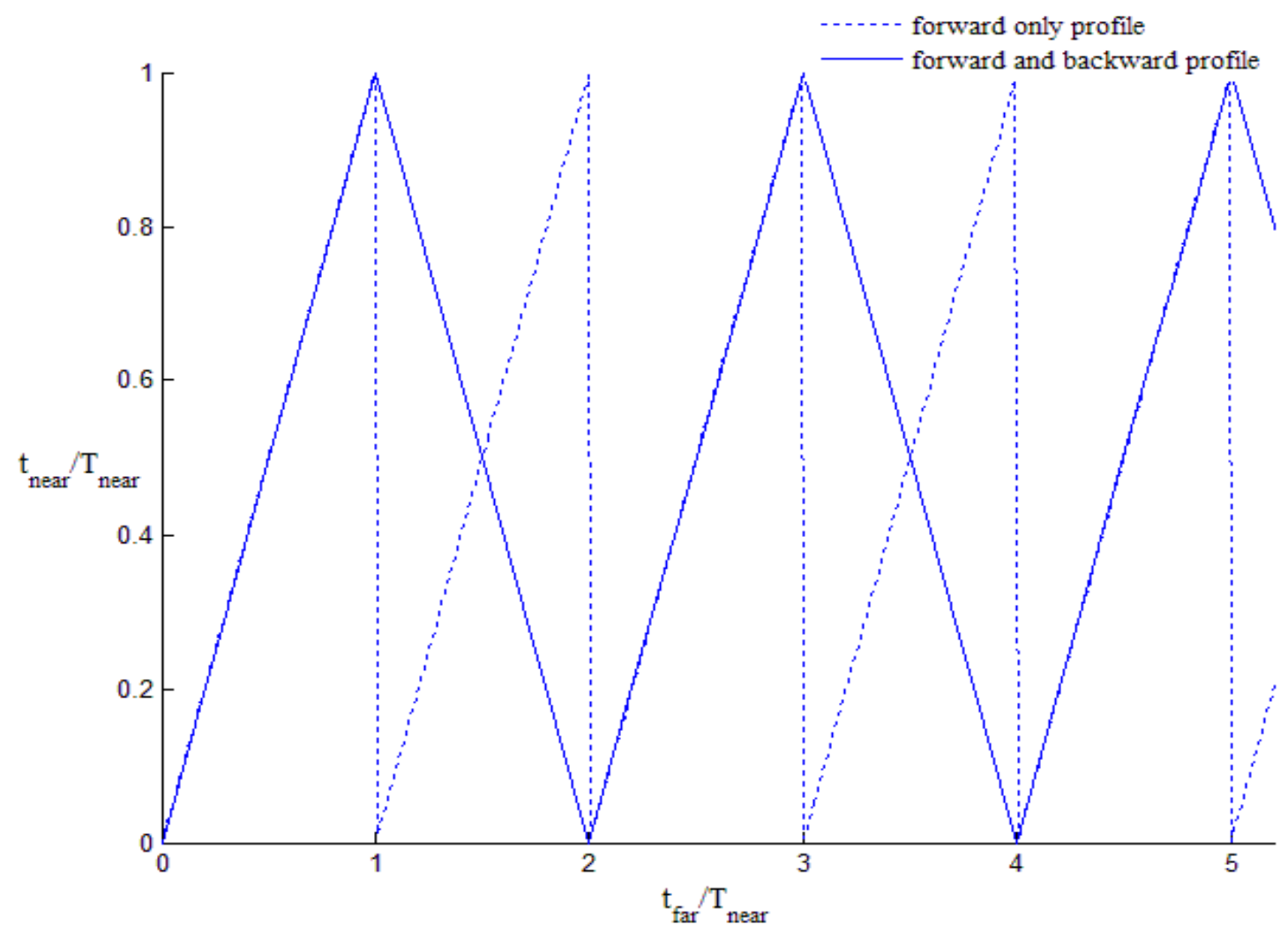

Figure 7.2.1. Time of the interface plane for the far-field $\left(t_{\mathrm{far}}\right)$ in terms of the recorded time in the near-field $\left(\mathrm{t}_{\text {near }}\right)$.

\subsection{Results and Comparisons to Previous Studies}

The instantaneous Mach number contours for the full-field simulations in an azimuthal plane are shown for the range where experimental data are available (Fig. 7.3.1a). In this region the flow spreads and the velocity decreases. In the range corresponding to the near-field domain (Fig. 7.3.1b), the flow is transonic with shock 
diamonds starting at the jet exit which dissipate due to turbulent structures that initiate from the thin shear layers at the jet edge. The near-field region flow complexity and high velocity gradients around the nozzle exit demonstrate the necessity for a fine grid within this area. A comparison of the time-averaged centerline velocity between the full-field LES and experiments can be seen in Fig. 7.3.2. The high velocity fluctuations within the near-field are due to the transonic region. These results indicate that the LES compares favorably to the experiments in terms of the downstream reduction in velocity. Further discussions on the time-step and grid independence studies for this flow are given by Lee $^{10}$.

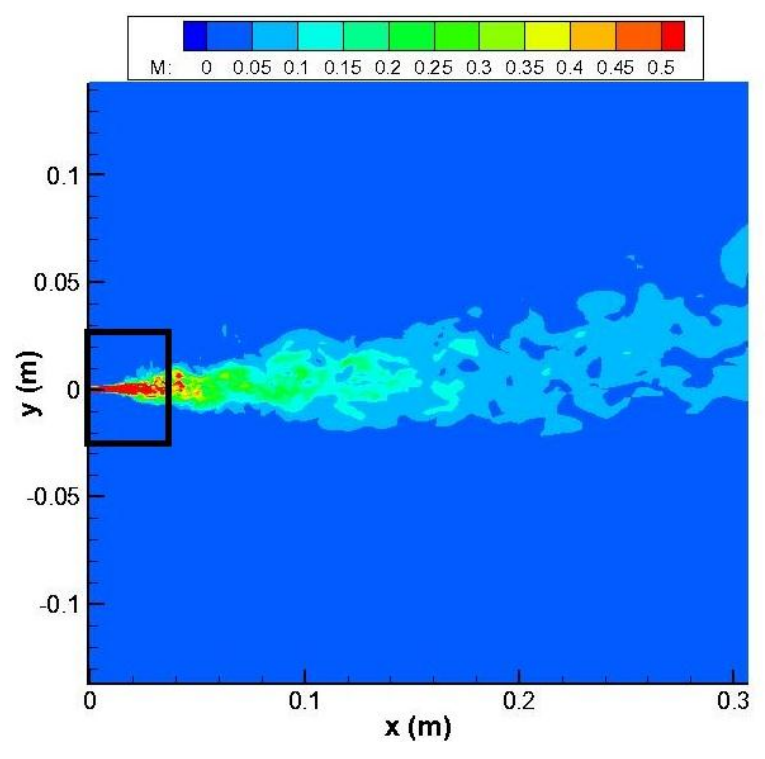

(a)

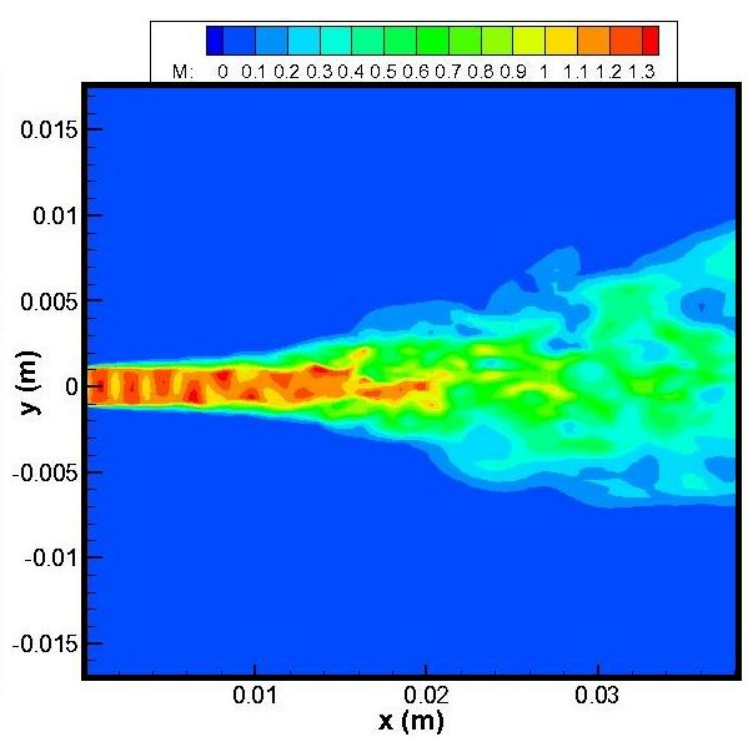

(b)

Figure 7.3.1. Instantaneous contours of Mach number from a planar slice of the full-field jet simulation where field of view corresponds to (a) region where experimental data is available with near-field region shown by a black box and (b) at near-field domain where flow is transonic. 


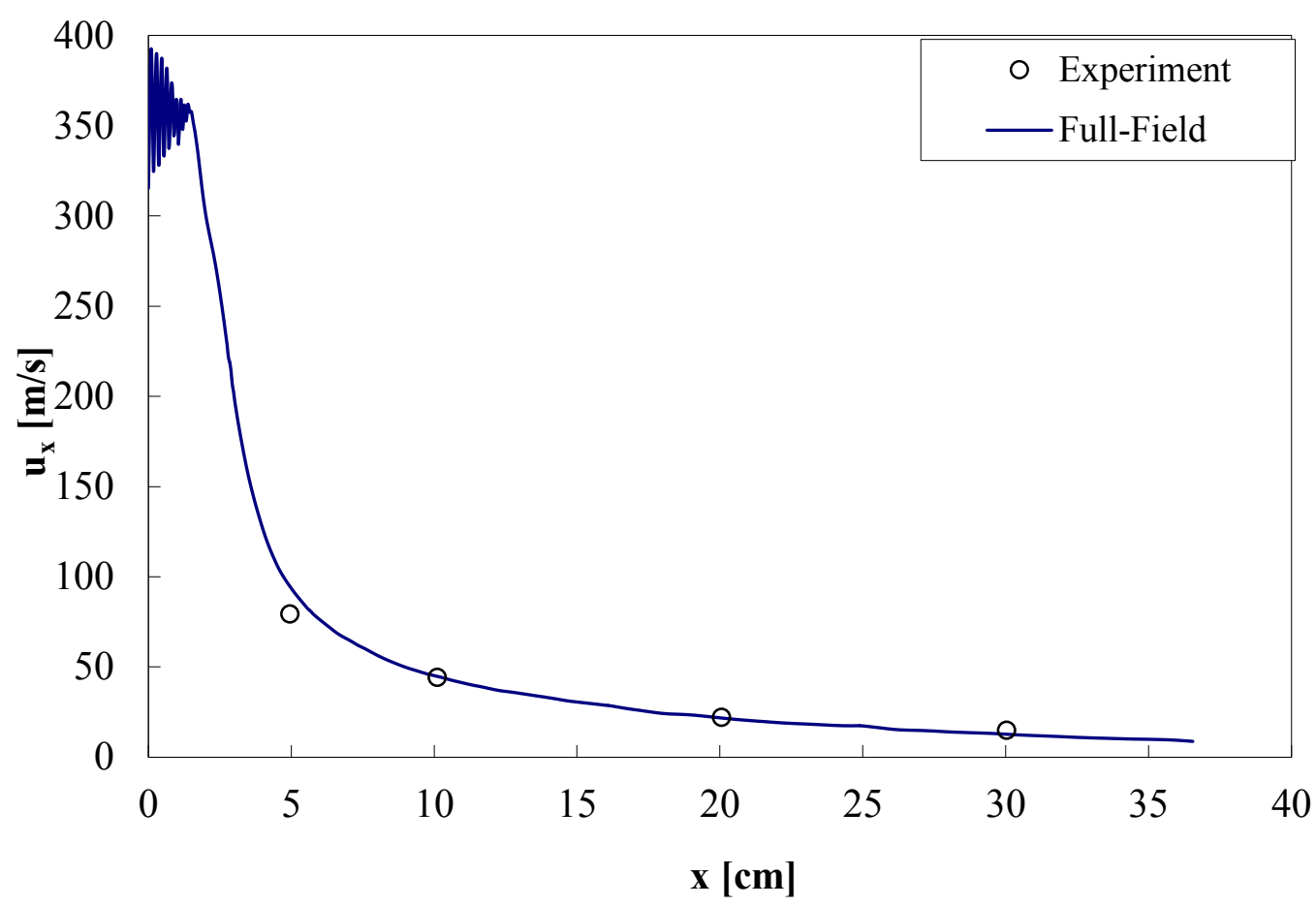

Figure 7.3.2. Comparison of centerline velocity between full-field simulation ${ }^{10}$ and experiment ${ }^{9}$.

Next we consider the far-field simulations using the unsteady interface coupling schemes discussed above. The instantaneous Mach numbers in an azimuthal plane for the far-field simulations using a Recorded Interface Boundary Condition (RIBC) with a forward and backward profile are displayed in Fig. 7.3.3a. Unsteady fluctuations captured by the RIBC are observed in the instantaneous contours as the jet flow moves downstream. The time-averaged Mach numbers (Fig. 7.3.3b) demonstrate that the simulated jet flow dissipates and reduces in velocity as it flows downstream, which was seen in the full-field simulations (Fig. 7.3.1a). The Mach number contours for a RIBC with a forward-only profile offer no noticeable differences from Fig. 7.3.3. 


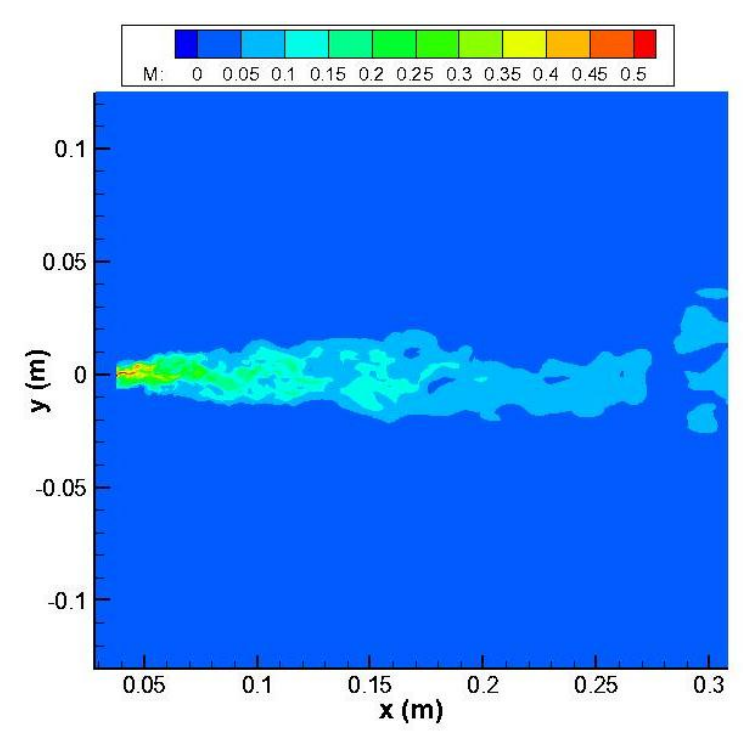

(a)

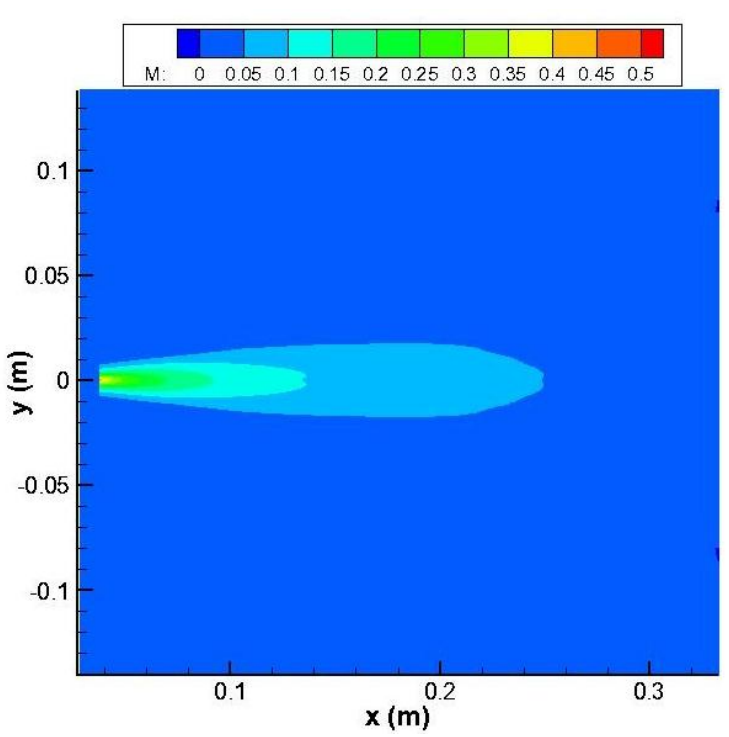

(b)

Figure 7.3.3. (a) Instantaneous and (b) time-averaged Mach number contours for farfield simulation using Recorded Interface Boundary Condition using a backward and forward profile.

A series of temperature contours were taken at the end of the near-field recording to highlight the differences between the forward-only and the forward and backward profiles for a RIBC (Fig. 7.3.4). The first two contours are taken immediately before the end of the recorded data from the near-field and are equal for the forward and backward profile (Fig. 7.3.4a) and the forward-only profile (Fig. 7.3.4b) because these simulations are on the first playback of the recording. The differences begin to appear at the third contour $\left(\mathrm{t}_{\text {far }} / \mathrm{T}_{\text {near }}=1\right)$ when the playback reaches the end of the recording and operates as discussed above (Fig. 7.2.1). The discontinuous temporal jump at $t_{\text {far }} / T_{\text {near }}=1$ for the forward-only profile causes the low temperature pocket at the jet center and similar phenomena that would not otherwise occur. It was found that after long time integrations this case could go unstable, which may be a result of the discontinuity in the flow fluctuations. The forward and backward profile demonstrates a smoother transition 
without any discontinuous changes to the flow characteristics. There may be errors propagated by the discontinuous jump in the time-derivative quantities like acceleration, but these were not observed in this study. The effect of these two variants on the downstream jet simulation will be discussed in the following through comparisons with experiments and full-field simulations. 


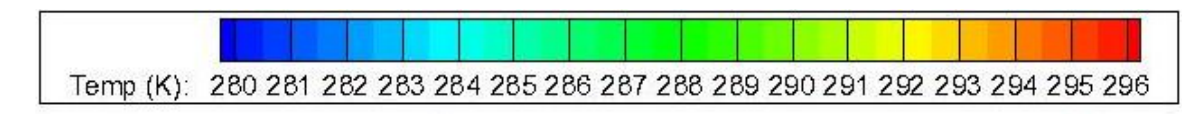

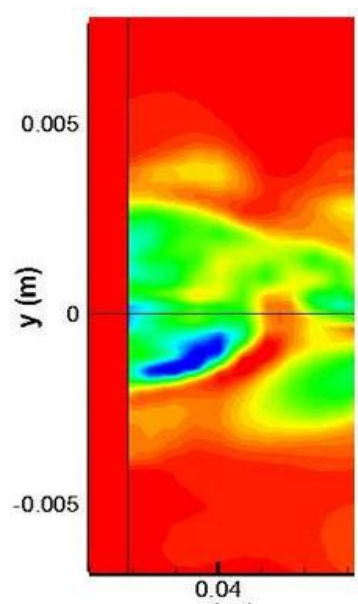

$x(m)$
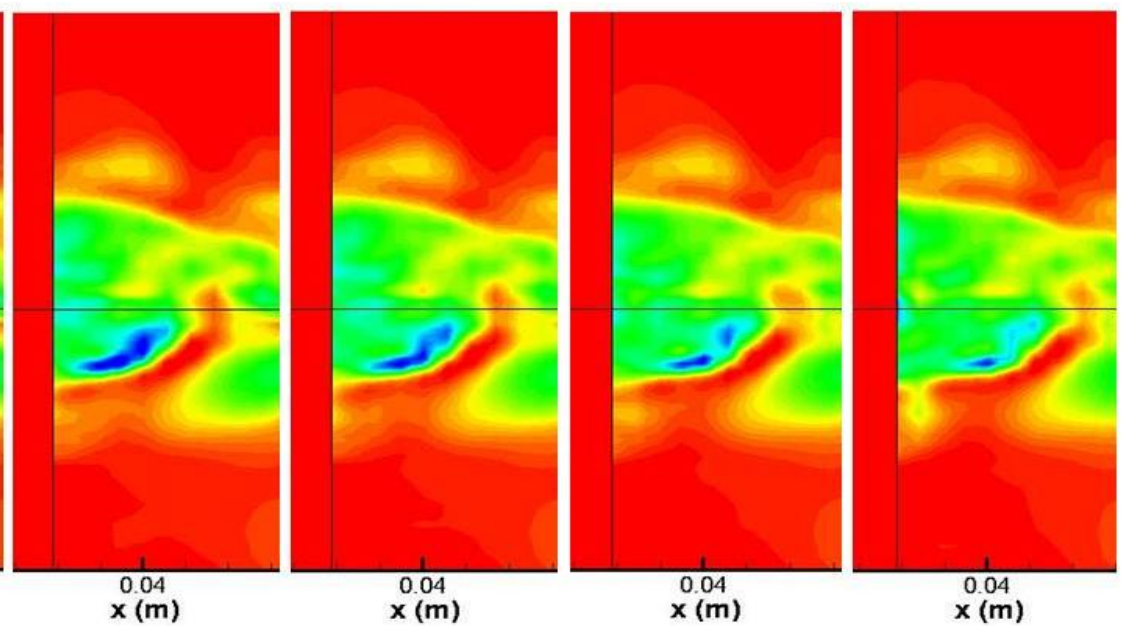

(a)
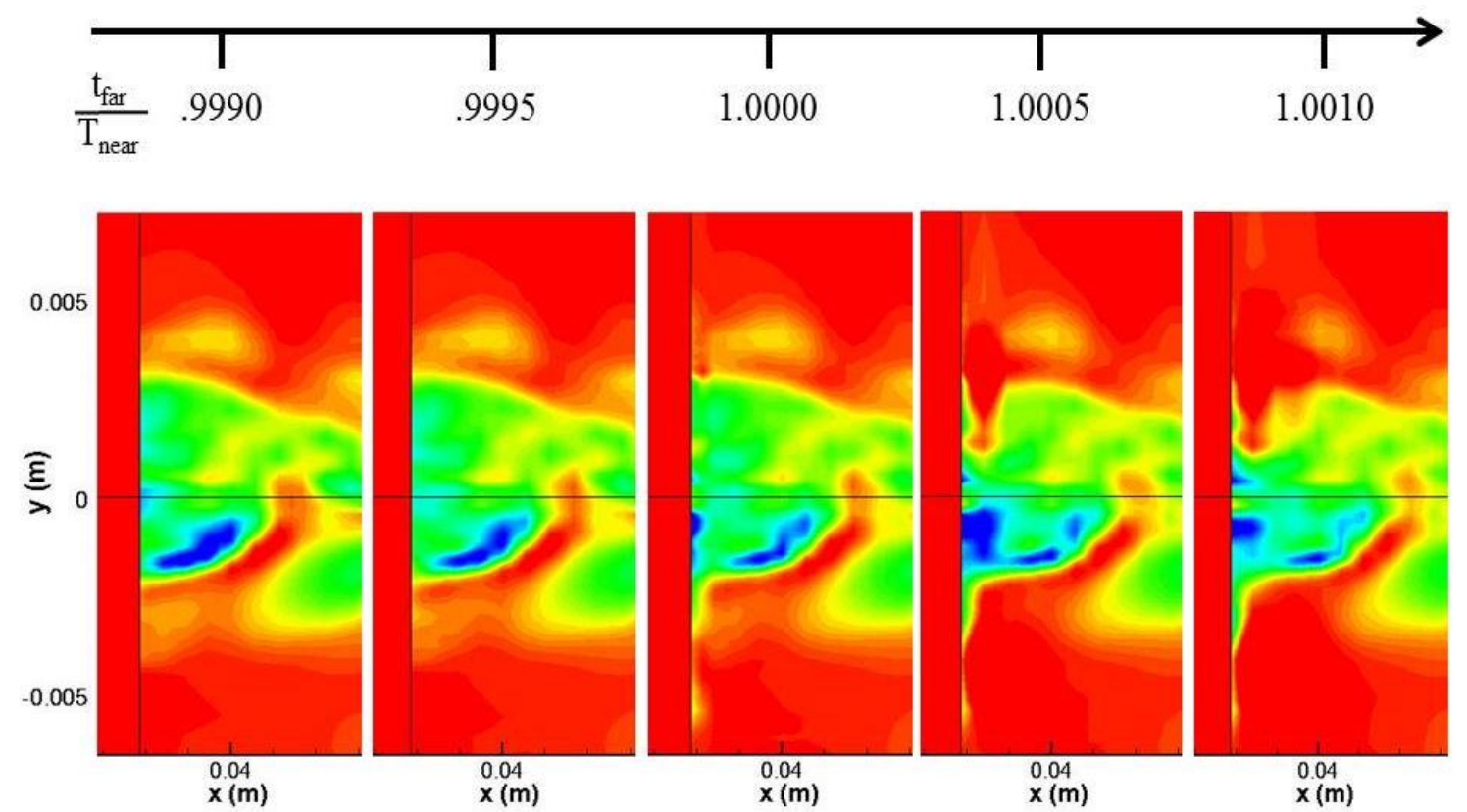

(b)

Figure 7.3.4 . Time sequence of temperature contours for far-field simulation close up on the interface using (a) forward and backward profile (b) forward-only profile. 
Time-averaged centerline velocity for the full-field simulations performed by Lee $^{10}$, experiments from Bulzan ${ }^{9}$ and far-field simulations using a Recorded Interface Boundary Condition are shown in Fig. 7.3.5. The forward and backward profile and the forward-only profile for the RIBC are shown starting at the far-field interface which avoids the velocity fluctuations due to transonic flow seen within the near-field in Fig. 7.3.2. Both profiles demonstrate favorable comparisons to the experiments and full-field simulations in terms of the mean velocity decreasing as the jet flows downstream. Timeaveraged velocities at $10 \mathrm{~cm}$ and $20 \mathrm{~cm}$ downstream of the nozzle are displayed in Fig. 7.3.6. The velocities for the far-field simulations differ more from experiments than the full-field simulation, however, the comparison is favorable with the maximum difference of approximately seven percent between the far-field and experiment. The centerline shows the largest disparities between the far-field simulations and full-field simulations and experiments. Radial distances above $15 \mathrm{~mm}$ at $10 \mathrm{~cm}$ downstream (Fig. 7.3.6a) and above $30 \mathrm{~mm}$ at $20 \mathrm{~cm}$ downstream (Fig. 7.3.6b) show small velocity disparities between each of the cases. There are no major velocity differences between the forward and backward profile and the forward-only profile, which demonstrates the errors generated from the two methods of handling the end of a recording, have minimal effect on the jet velocity. 


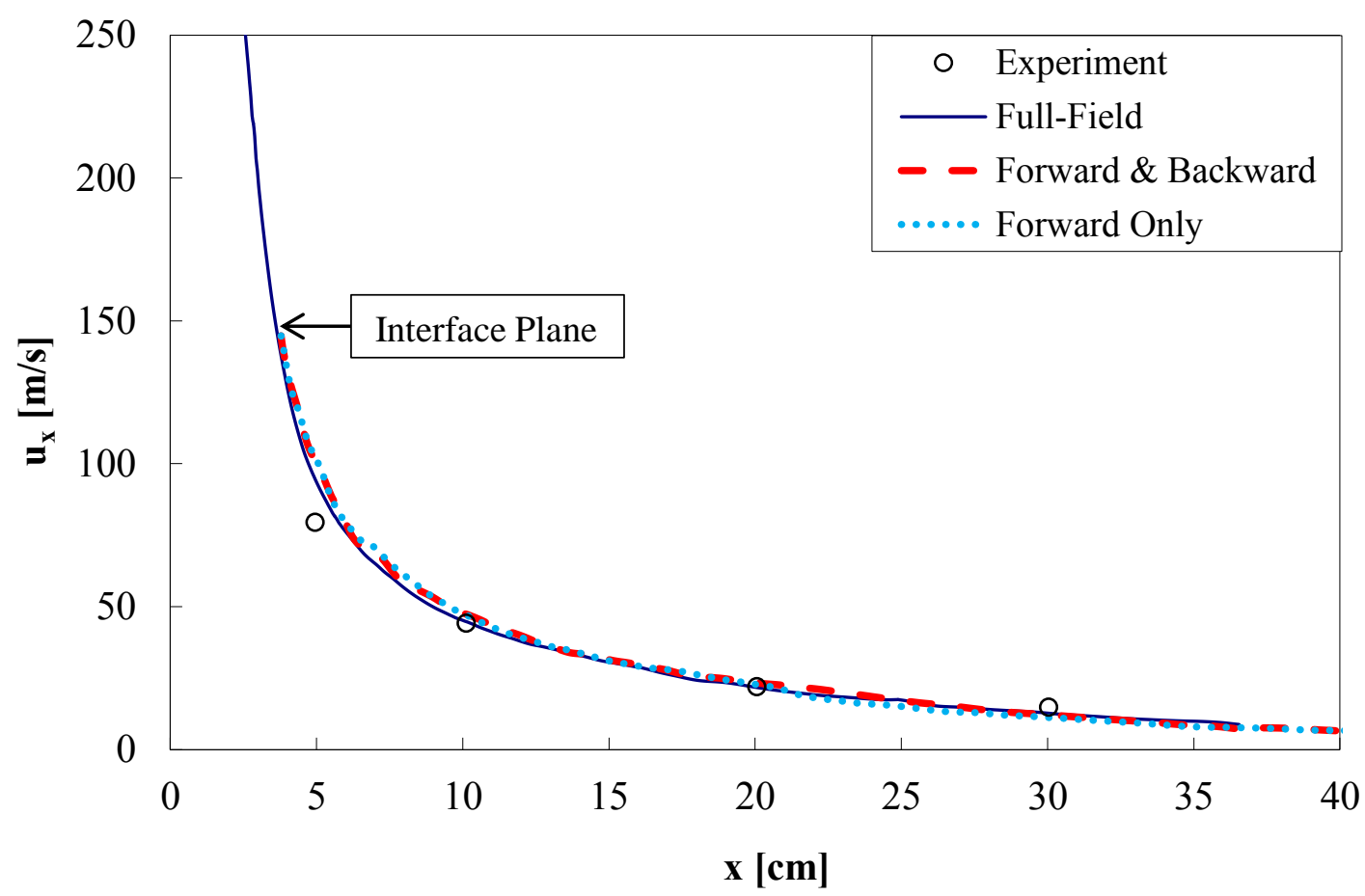

Figure 7.3.5. Comparison of time average centerline velocities between full-field simulation $^{10}$, experiments ${ }^{9}$ and far-field simulations with a RIBC using forward and backward and forward-only profiles. 


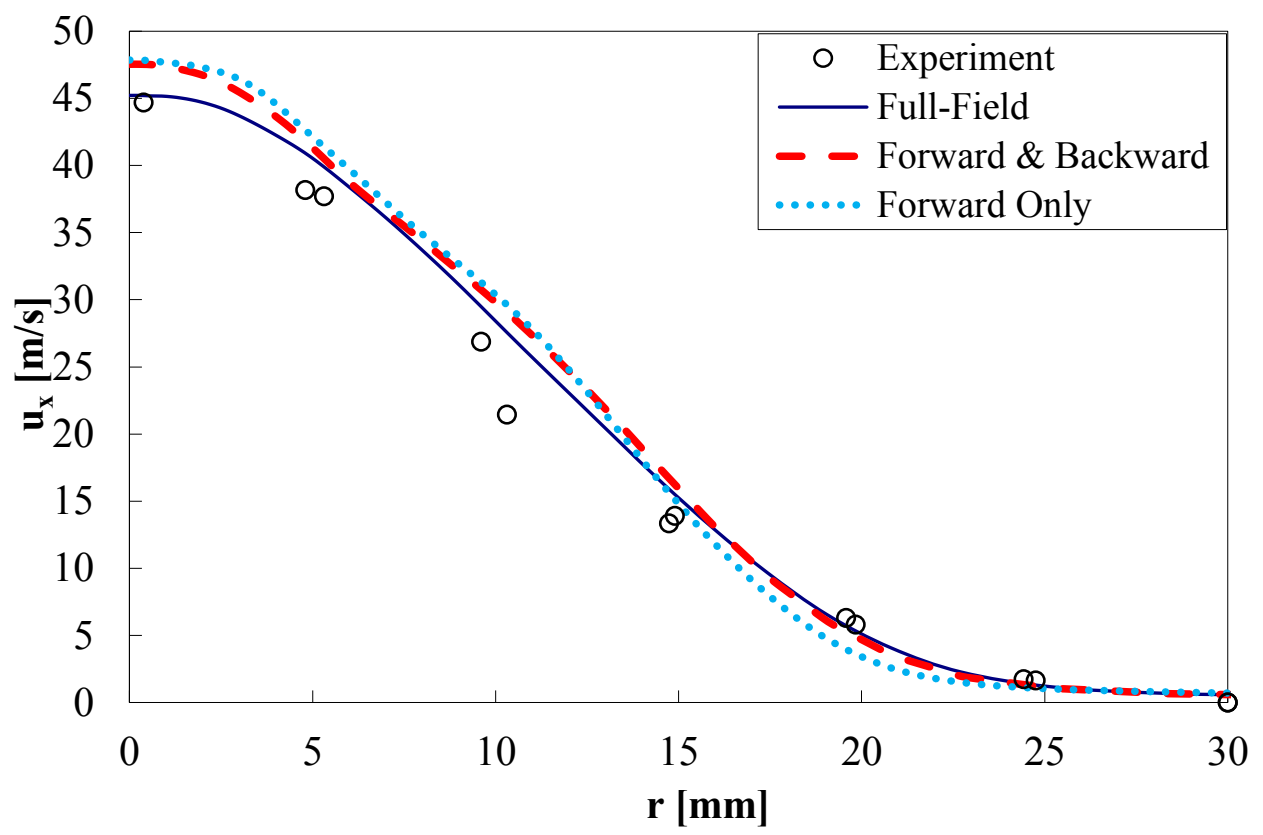

(a)

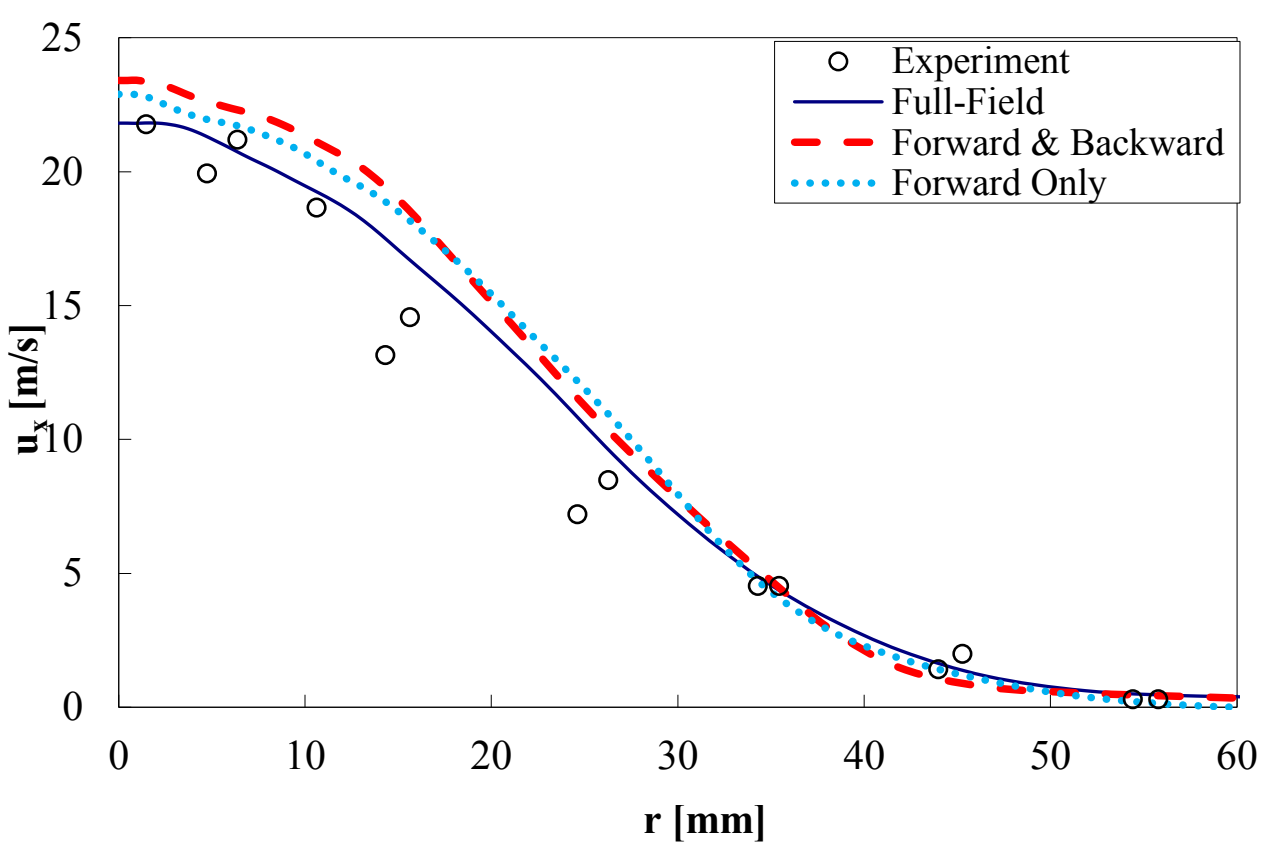

(b)

Figure 7.3.6. Radial comparison of time average velocities between experiment ${ }^{9}$, fullfield simulations ${ }^{10}$ and far-field simulation with the two variants of the RIBC at (a) $10 \mathrm{~cm}$ downstream, (b) $20 \mathrm{~cm}$ downstream. 
The different turbulent kinetic energy components discussed in the methods section are shown for the far-field simulation $10 \mathrm{~cm}$ downstream of the nozzle exit (Fig. 7.3.7a). The total turbulent kinetic energy is the sum of the resolved turbulent kinetic energy and the sub-grid turbulent kinetic energy. The resolved turbulent kinetic energy from the root mean square of the velocity fluctuations comprises the majority of the total turbulent kinetic energy seen in the LES simulations. Figure 7.3.7b displays the total turbulent kinetic energy from experiments, full-field simulation and far-field simulations using the RIBC with either the forward and backward profile or the forward-only profile. The far-field simulations are producing slightly lower turbulent kinetic energy then seen experimentally, but match up favorably with predictions done by full-field simulations. The singular axis boundary condition specified at the center of the jet is hypothesized as the reason for the turbulent kinetic energy in the LES simulations to differ from experiments more at the centerline than the rest of the simulation. 


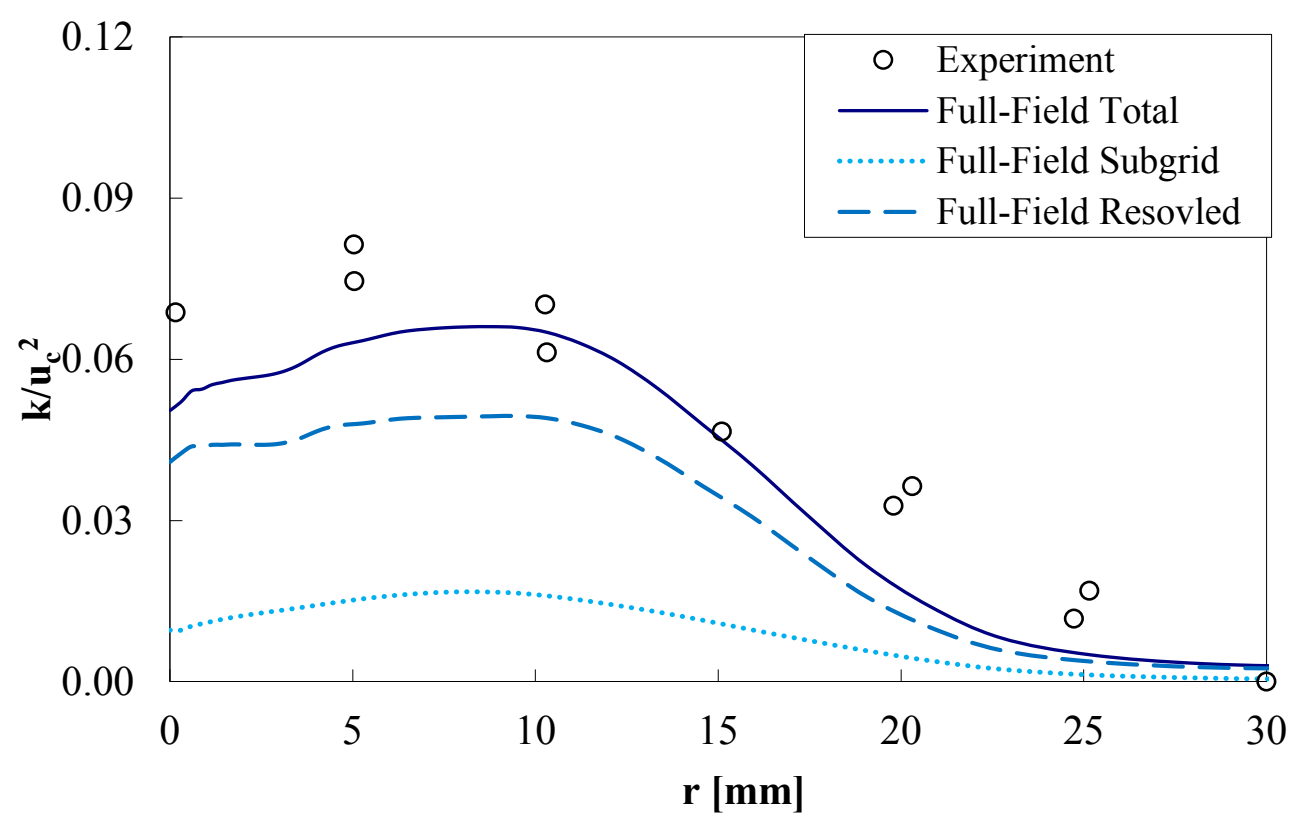

(a)

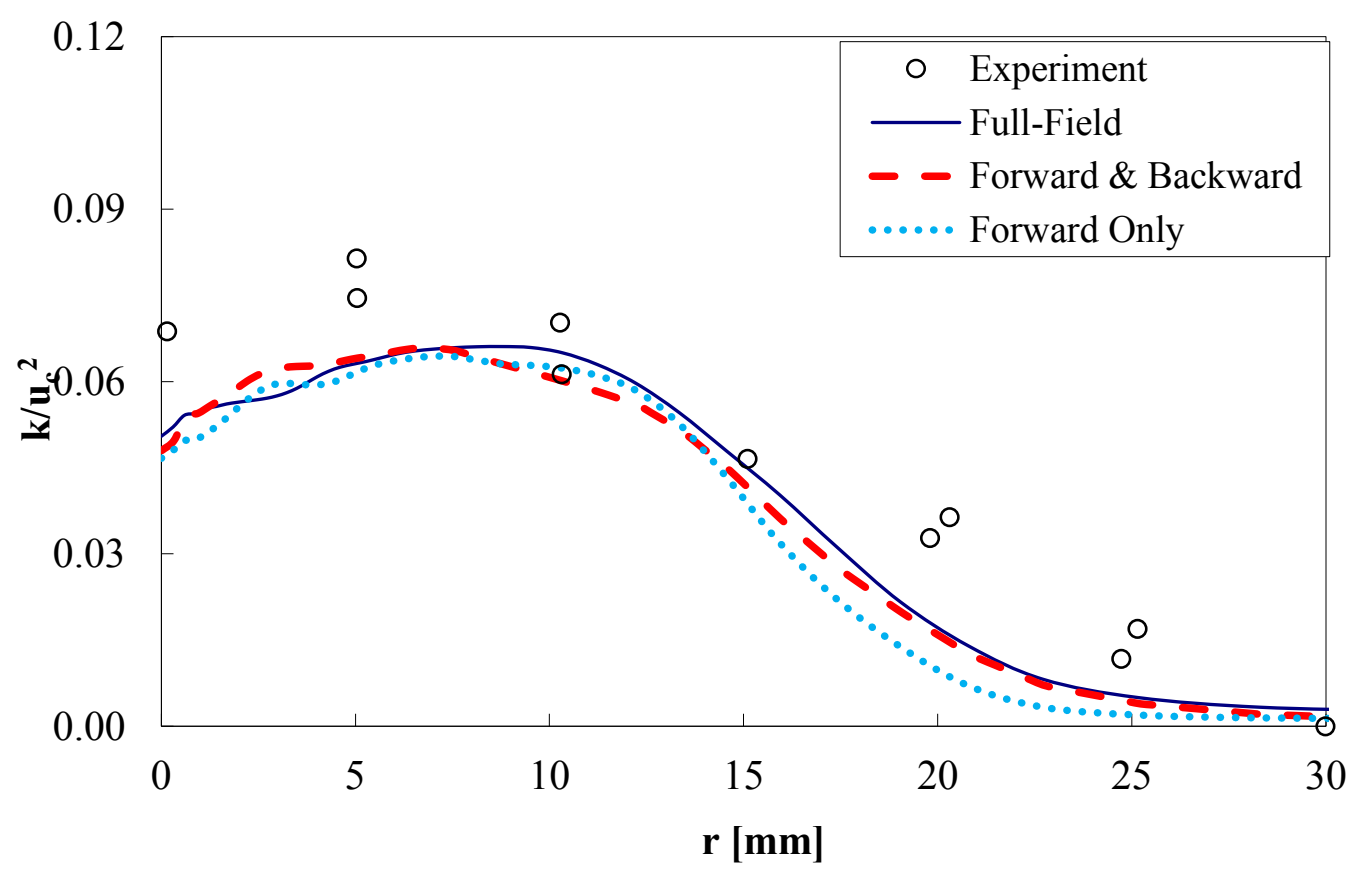

(b)

Figure 7.3.7. Radial comparison at $10 \mathrm{~cm}$ of (a) the different components of turbulence for the full-field simulation and experiments (b) the total turbulence for experiments ${ }^{9}$, full-field simulations ${ }^{10}$ and far-field simulation with the two variants of the RIBC. 
Figure 7.3.8a presents the root mean squares of the velocity fluctuations in each direction for both the full-field case and the far-field case using the two variants of the Recorded Interface Boundary Condition. The streamwise velocity fluctuations $\left(\mathrm{u}_{\mathrm{x}, \mathrm{rms}}\right)$ are around six times higher when compared to the radial fluctuations at the centerline for both the full-field and the far-field simulations. This means that the resolved turbulent kinetic energy seen in Fig. 7.3.7 is mostly comprised of the streamwise fluctuations and since the majority of the total turbulent kinetic energy is resolved turbulent kinetic energy, the bulk of the turbulent kinetic energy is generated by the streamwise fluctuations. Figure 7.3.8(b) shows the root mean square of the streamwise fluctuations for $20 \mathrm{~cm}$ downstream. The far-field simulations are consistent with the full-field simulations for both the forward and backward and the forward-only profile. These turbulent kinetic energy comparison figures illustrate that the multi-scale method is simulating the jet stream relatively well when compared to experiments and very well relative to the full-field simulation. The figures also show that the overall time-average results for the far-field jet stream are not affected by the two different jet profiles.

The RIBC within the far-field is generating appropriate jet physics downstream based off velocity and turbulence comparisons to the experiments done by Bulzan ${ }^{9}$ and full-field simulations by $\mathrm{Lee}^{10}$. This demonstrates that the majority of the flow characteristics are being transferred properly from the near-field domain to the far-field domain. Both the forward and backward profile and the forward-only profile compare favorably to the experiments and full-field simulations. However, the forward and backward profile tends to be more stable than the forward-only profile and is the 
recommended technique for implantation into the hybrid RANS/LES simulations of the spray bar region.

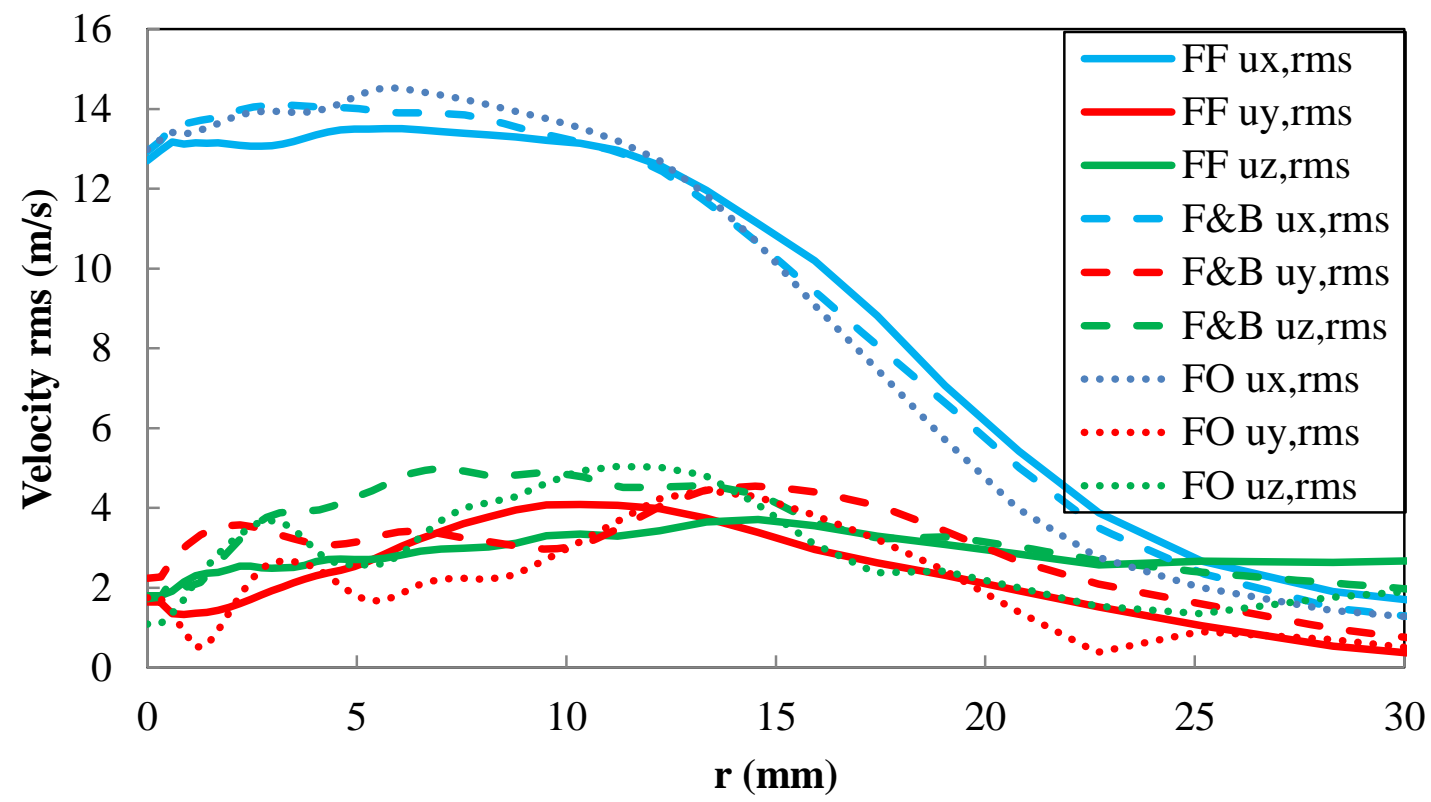

(a)

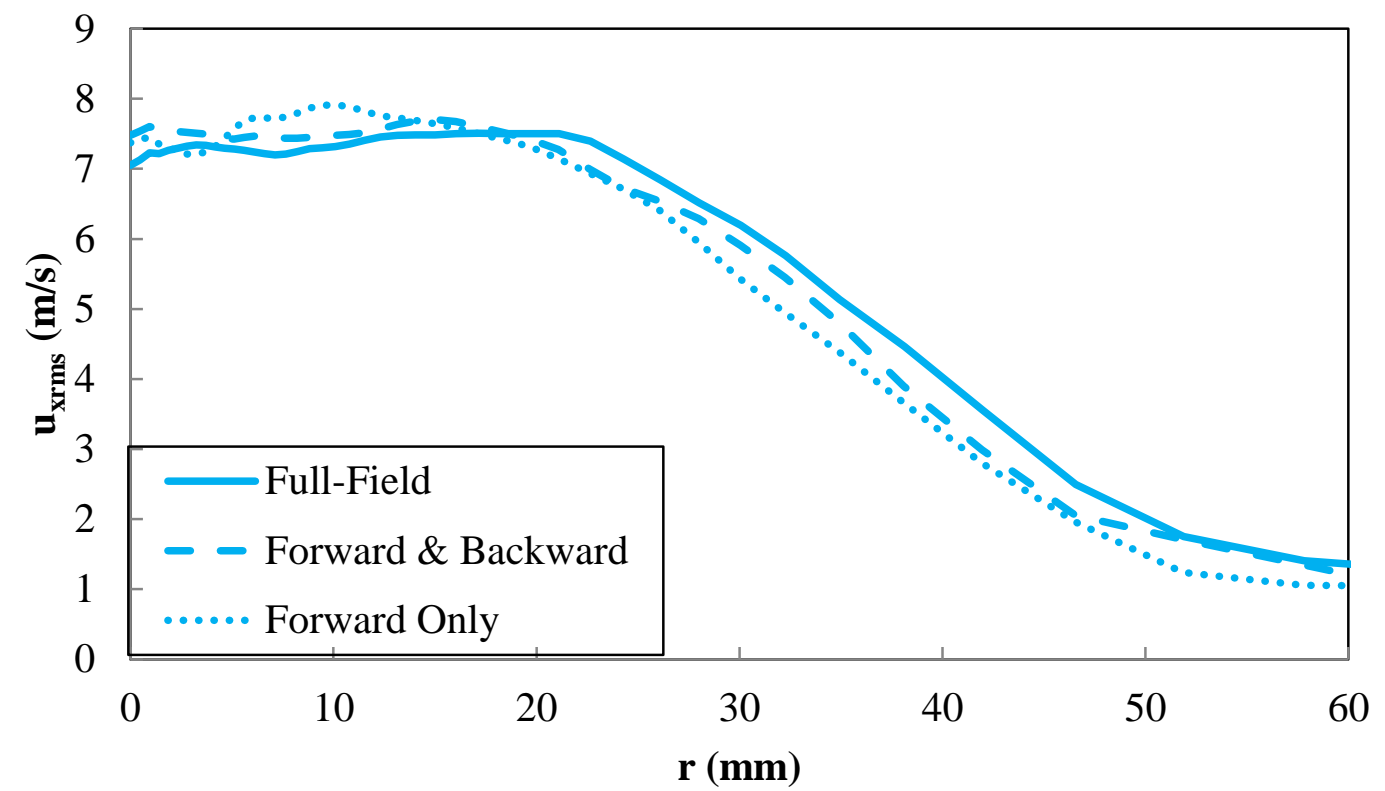

(b)

Figure 7.3.8. Radial comparison between full-field simulations ${ }^{10}$ (FF) and far-field simulations with the RIBC using the forward and backward profile (F\&B) or forwardonly profile (FO) at (a) $10 \mathrm{~cm}$ downstream with all velocities rms (b) $20 \mathrm{~cm}$ downstream with axial velocity rms. 


\section{Chapter 8. Single Spray Bar Hybrid RANS/LES Simulation}

\subsection{Computational Domain}

A simulation of the entire NASA Glenn IRT domain with a hybrid RANS/LES model would be too impractical with the computational resources currently available. Instead the hybrid RANS/LES model can be used to focus on the near spray bar region allowing the model to simulate the flow physics near the nozzle exit where jet flows initiate. Focusing on this area will allow for analysis of the unsteady fluctuations within the jet flows without having to simulate the entire tunnel. For the current study a base simulation was developed for a single spray bar without any active nozzles or vertical struts.

The base simulation has a computational domain that reaches from 58" upstream of the spray bar to 27.5 " downstream where the NASA IRT starts its contraction towards the test section. A coarser domain was incorporated after the 27.5 "to extend the simulation another 50" insuring that any outlet boundary conditions would not be affecting the flow around the spray bar. The simulation has a length of 18 " along the spanwise direction and a vertical distance of 24". The span length was chosen to allow for multiple nozzles to fit in the computational domain and the vertical distance was chosen because of the 12" spacing between spray bars. Figure 8.1.1 shows a side view of the entire single spray bar computational domain.

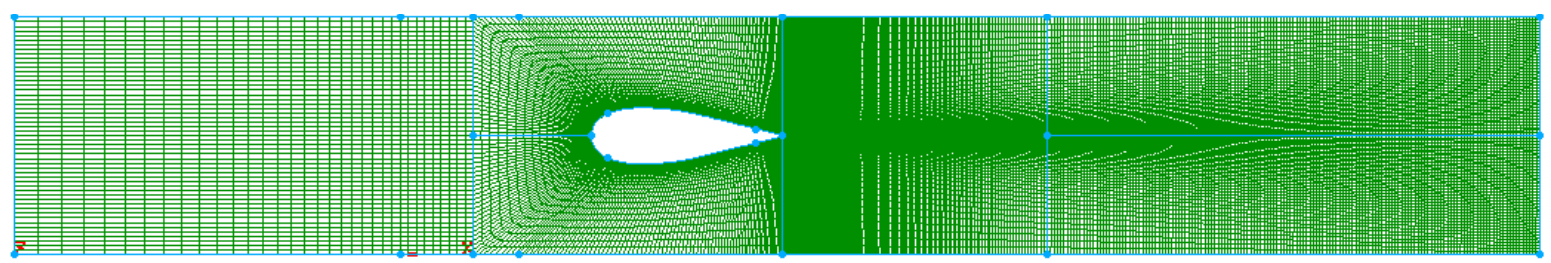

Figure 8.1.1. Side view of the computational domain of the single spray bar simulation. 
The domain was separated into four different zones to allow for multiple computer processors to calculate simultaneously. As seen in Fig. 8.1.1 the four zones are separated so that there is one zone upstream, two zones downstream and a single zone surrounding the spray bar. The two zones downstream are separated 27.5 " downstream of the spray bar where the IRT would start its contraction. The computational zones upstream and downstream of the spray bar are structured rectangular grids while the zone surrounding the spray bar is a structured C-grid.

The four different computational zones have a total of 2.4 million cells. Figure 8.1.1 illustrates that the finest spacing is located near the spray bar and immediately downstream of the trailing edge while the domain coarsens far upstream and downstream. The top, bottom, and side boundaries use an slip wall boundary condition. The front left boundary is an arbitrary inflow condition with atmospheric conditions, a Mach number of 0.1 , and turbulence values of $\mathrm{k}=.528 \mathrm{ft}^{2} / \mathrm{s}^{2}$ and $\omega=4.816 \mathrm{~s}^{-1}$ obtained from studies performed by Bhargava ${ }^{4}$. The end of the simulation is an outflow boundary condition set to atmospheric conditions.

\section{2: Single Spray Bar Results}

The wake shedding and instantaneous fluctuations simulated behind the spray bar using the hybrid RANS/LES model can be seen in Fig.8.2.1. This figure displays instantaneous contours of the streamwise velocity and vorticity magnitude from a side perspective. The flow starts to separate and generate vortices between half way and the last quarter of the spray bar. The vorticity magnitude is very sensitive to flow fluctuations and highlights the location of the vortices as they travel downstream. As the vortices move they grow and become larger than the height of the spray bar as they reach 
the start of the tunnel contraction. The black line 27.5 " downstream of the spray bar in Fig. 8.2.1 represents the start of the tunnel contraction. The previous RANS simulations of the spray bar within the NASA Glenn IRT would not simulate these vortices and flow separation.

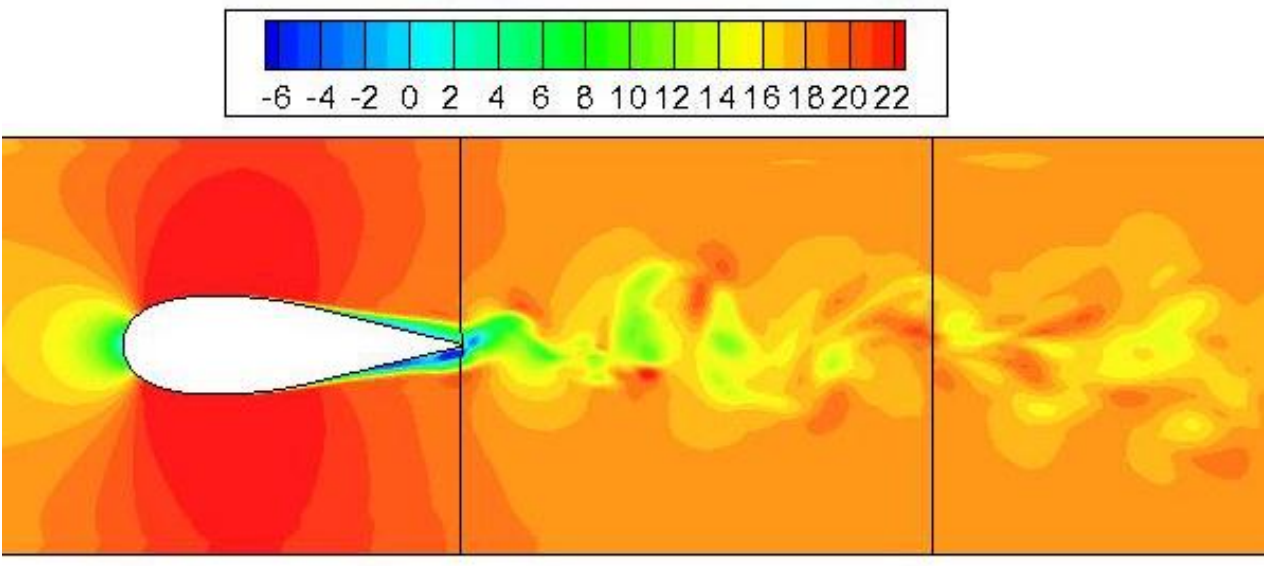

(a)
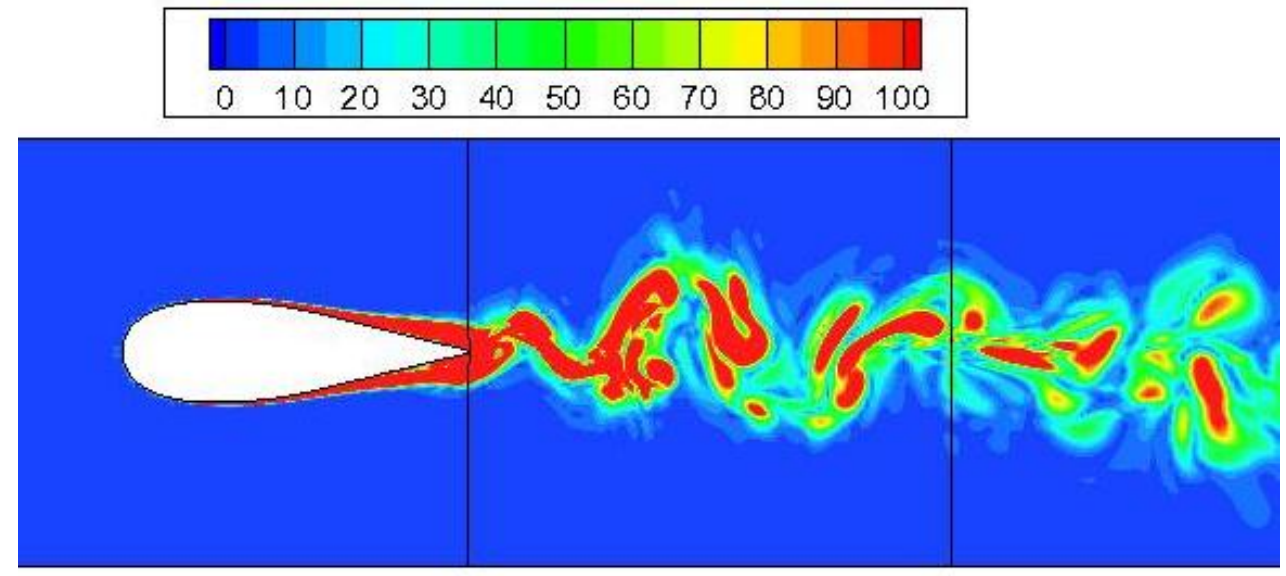

(b)

Figure 8.2.1. An instantaneous snapshot of the (a) streamwise velocity in $\mathrm{ft} / \mathrm{s}$ and (b) vorticity magnitude in $\mathrm{s}^{-1}$ of the single spray bar RANS/LES simulation.

Figure 8.2.2 shows the time averaged streamwise velocity for the hybrid RANS/LES simulation and the quarter-tunnel RANS simulation of the 2000 IRT configuration. The time-averaged contours for the hybrid RANS/LES assume that there is vertical symmetry downstream of the spray bar. The negative velocity near the spray bar 
trailing edge demonstrates that the hybrid RANS/LES is predicting some recirculation in this area. The pure RANS simulation has a smaller wake than the hybrid RANS/LES simulation. The RANS simulation does include the tunnel contraction which explains why it increases in velocity near the right side and has a slight angle of attack. However, the lack of any indication of flow recirculation and the reduction in wake size indicate that the pure RANS simulation is missing some key flow characteristics around the spray bar.

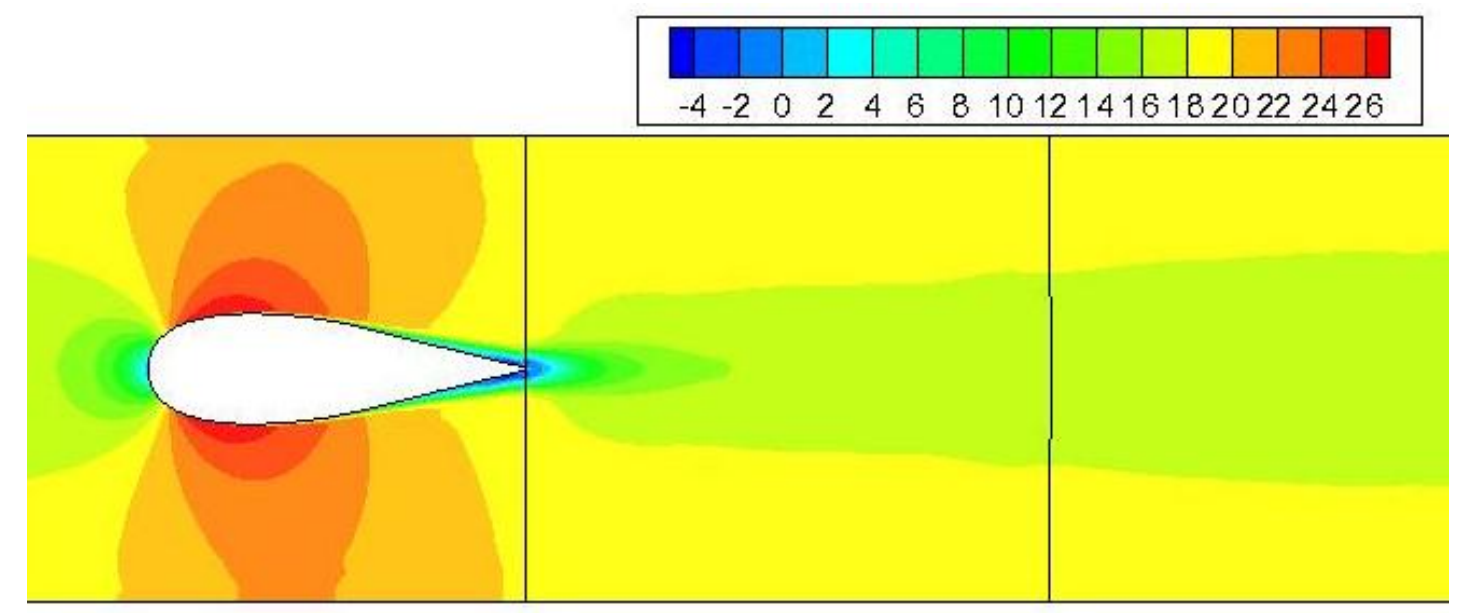

(a)

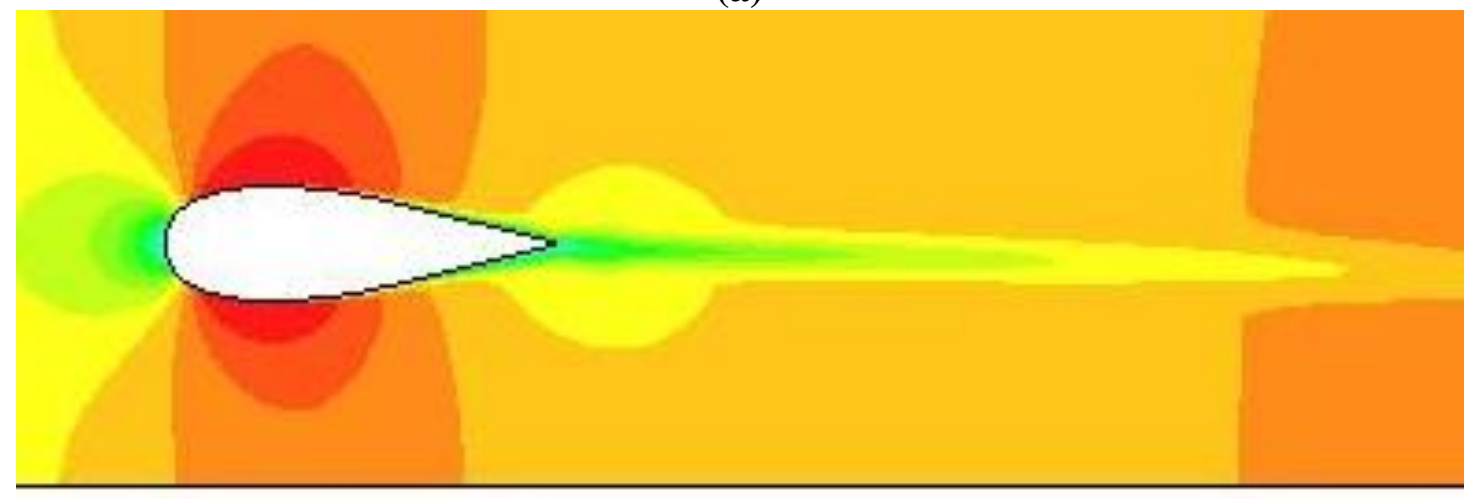

(b)

Figure 8.2.2. Contours of time averaged axial velocity, $\mathrm{u}_{\mathrm{x}}$, in $\mathrm{ft} / \mathrm{s}$ around a single spray bar for (a) hybrid RANS/LES simulation and (b) RANS simulation of quarter-tunnel IRT. 
Figure 8.2.3 shows the time-averaged total turbulent kinetic energy (i.e. the sum of the sub-grid and resolved turbulent kinetic energy) for the hybrid RANS/LES simulation and the quarter-tunnel RANS simulation of the 2000 IRT. The turbulent kinetic energy starts high near the trailing edge and quickly decreases as the flow moves downstream. The turbulent kinetic energy for the RANS simulations is much lower than the hybrid RANS/LES simulations because the RANS simulation does not capture any shedding effects. The majority of the turbulent kinetic energy generated within the wake of the hybrid RANS/LES simulation comes from the flow separation and shedding. The size of the wake behind the spray bar is also much smaller than the hybrid RANS/LES model which was also seen in Fig. 8.2.2. The hybrid RANS/LES method allows for the growth of the vortices which incorporate more area downstream than what is modeled in the RANS simulation. Due to the effect turbulence has on the water droplet dissipation, the LWC within the NASA Glenn IRT RANS simulations may be higher than reality, demonstrating the need for the hybrid model to analyze the area near the spray bar. 

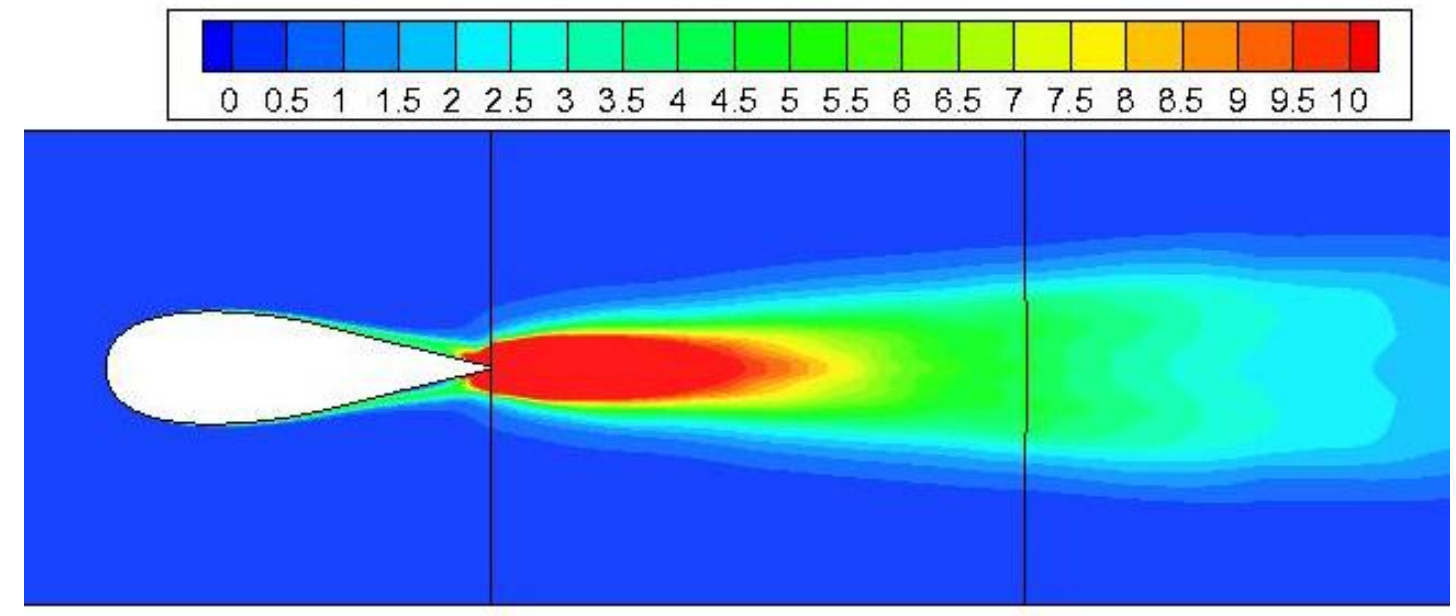

(a)

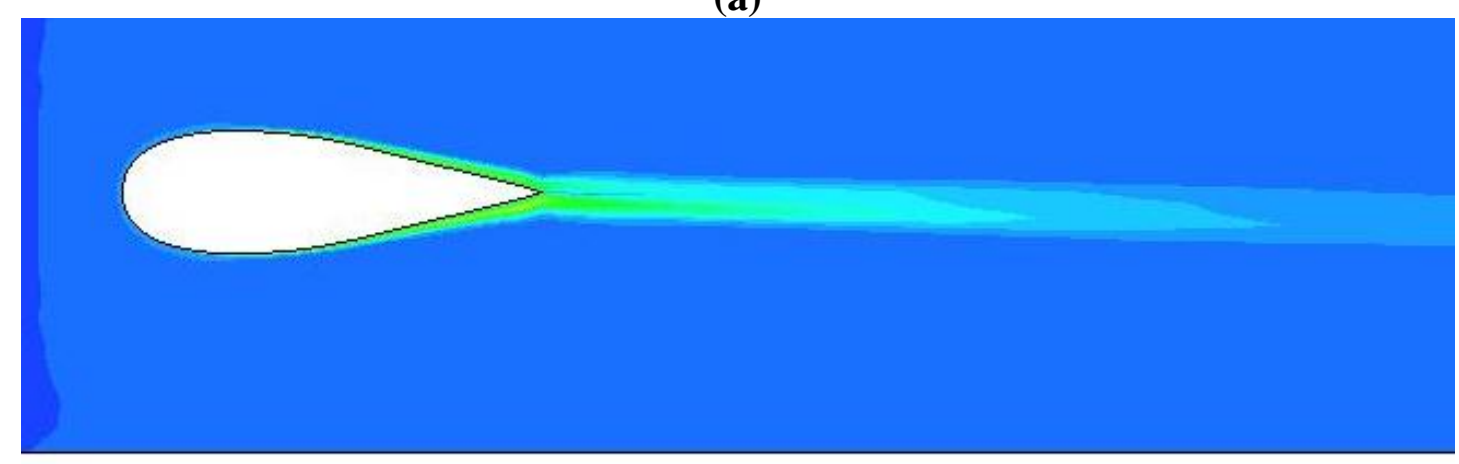

(b)

Figure 8.2.3. Contours of time average turbulent kinetic energy, $\mathrm{k}$, in $\mathrm{ft}^{2} / \mathrm{s}^{2}$ around a single spray bar for (a) hybrid RANS/LES simulation and (b) RANS simulation of quarter-tunnel IRT. 


\section{Chapter 9. Summary and Recommendations for Future Work}

\subsection{Summary}

A single spray bar simulation and a new jet model utilizing a Recorded Interface Boundary Condition (RIBC) were developed for future hybrid RANS/LES simulations of the spray bar region. The hybrid RANS/LES method can simulate the unsteady fluctuations and flow separation generated at the spray bar region which may allow for analysis of the "dancing" behavior observed in the video recording of certain NASA IRT water nozzles. Developing a new jet model that captures the flow instabilities generated at the nozzle exit while still being computationally reasonable to simulate far downstream (far-field) was an emphasis because capturing the "dancing" phenomenon requires proper calculations of the instantaneous flow fluctuations.

The process of separately computing the near-field and far-field jet domains with a RIBC was demonstrated for an axisymmetric round jet exhausting air more than 60 diameters downstream. This allows the far-field domain to be computed with much larger time-steps while still assuring computational accuracy and stability. The RIBC technique greatly reduces the computational demand for LES, with little impact on the fidelity of the predicted flow characteristics. Both the forward-only profile and the forward and backward profiles can be implemented, however the forward-only profile tends to have more problems maintaining stability than the forward and backward profile. As such, the forward and backward profile is recommended for any future implementation into hybrid RANS/LES simulations of the spray bar region.

A single spray bar simulation was developed with finer resolution and improved geometry to better match with the NASA Glenn IRT than the simulations performed by Lee $^{10}$. The predicted flow solution shows the generation of wake shedding and flow 
instabilities that were not captured within the RANS simulations of the IRT. As such, the hybrid RANS/LES simulation predicted higher turbulent kinetic energy and a larger wake than the RANS simulations. Any "dancing" behavior that occurs from flow instabilities generated by the interaction of a jet, the wake behind a spray bar, and any other tunnel component should be appropriately captured by the hybrid RANS/LES simulation.

\subsection{Recommendations for Future Work}

The single spray bar simulation should be utilized as a base computational domain to add jets using the RIBC, vertical struts, and other modifications to generate the "dancing" phenomenon. Incorporating a vertical strut into the spray bar simulations will allow for comparisons between only having a spray bar and the presence of a vertical strut. The RANS simulations of the IRT demonstrated that the flow turbulence increases greatly in magnitude when the vertical struts are installed especially in a wake behind an intersection of a spray bar and strut. The hybrid RANS/LES simulations will illustrate the details from this intersection and simulate the higher velocity fluctuations generated in the wake. Including a RIBC to model a single or multiple active nozzles will give the simulations the opportunity to demonstrate the "dancing" behavior. It is expected that the RANS/LES simulation that includes the vertical strut will simulate jets with higher "dancing" behavior as noticed in the recorded video of the NASA Glenn IRT nozzles.

Currently the RIBC only models the continuous flow generated from the jet nearfield and does not include any multiphase physics that will occur when a nozzle releases both air and water. Properly modeling the water breakup and then tracking the droplets trajectories will have to be developed in full-field and near-field jet simulations. The water droplet position and velocities at the coupling interface between the near- and far- 
field could be done either as a time-average profile or a recording similar to the continuous phase flow in a RIBC. Performing another recording may cause computational memory issues because of the high quantity of droplets that can pass the interface plane in a relatively short amount of time. Both time-average and recorded profiles should be investigated to determine the best way to model the water droplets with the resources available.

Water droplets should be released in the spray bar RANS/LES simulations with and without a vertical strut once the most appropriate model for releasing water droplets is determined. Releasing and tracking the water droplet will give insight into the effects these high fluctuations and "dancing" behavior will have on the droplet distribution near the spray bar. Knowing both the development of these jet fluctuations and their effects on the water droplets will assist in modifying the spray bar to generate these fluctuations without the need for a vertical strut. Since the vertical struts cause issues for spray bar maintenance and possible tunnel blockage, tunnel engineers desire a new spray bar geometry that could be implemented relative easily while still assisting in droplet distribution. Trailing edge flaps seen as the blue rectangles in the drawing looking upstream to the spray bars (Fig. 9.2.1a) are one idea to generate turbulence near the nozzle exits. The bluff spray bars in Fig. 9.2.1b are another idea that would generate greater flow separation upstream of the nozzle exits. These examples are two designs that could possibly generate the "dancing" phenomenon and should be investigated along with other ideas designed during future studies which should include all tunnel test conditions (flow speed, drop sizes, etc.). 


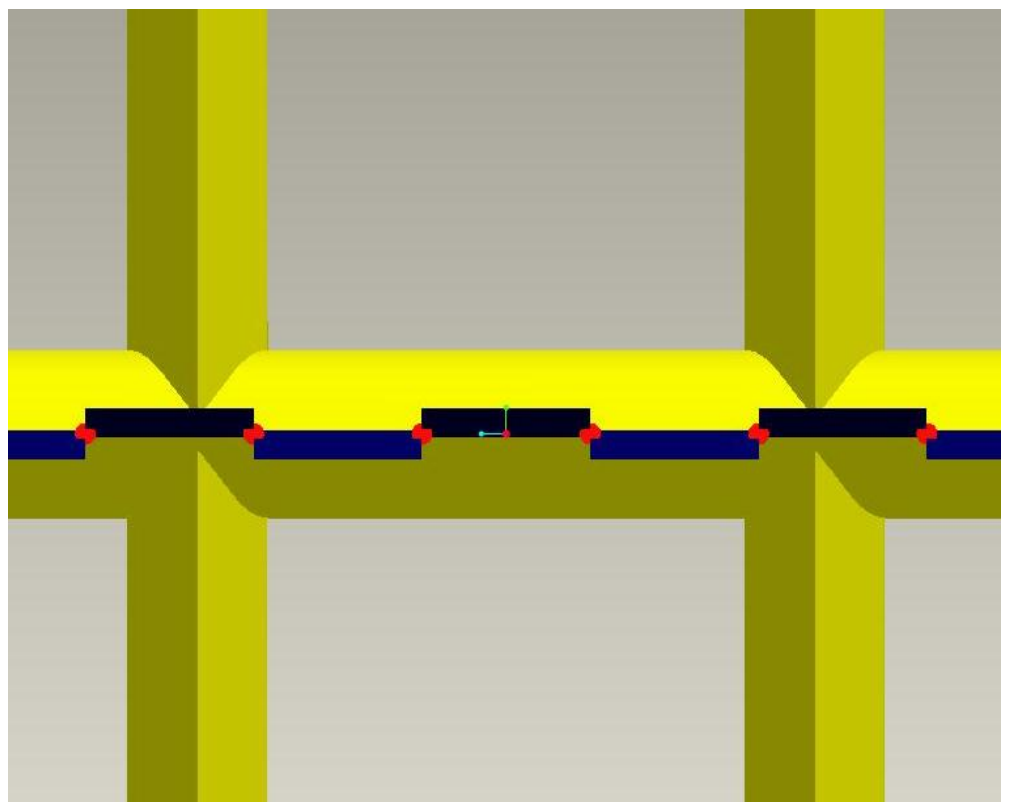

(a)

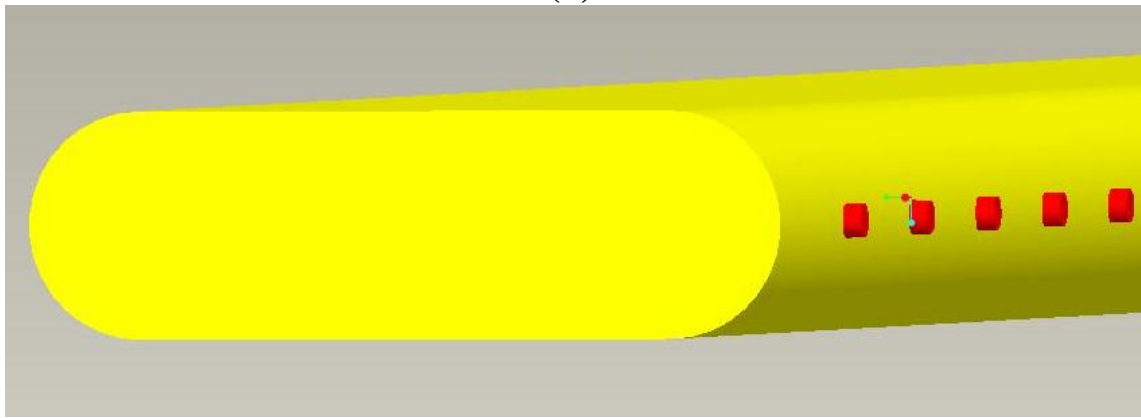

(b)

Figure 9.2.1: Examples of spray bar modifications: (a) alternating flaps seen as the blue rectangles and (b) bluff spray bar.

To summarize, future simulations should keep with the objective of analyzing the velocity fluctuations (i.e. "dancing" behavior) in the jet flow released from certain water nozzles. The recommended plan with the current perspective of the model and objective goes as follows:

1) Incorporate a vertical strut into the spray bar only simulation.

2) Include the RIBC to simulate multiple jet flows behind a single spray bar with and without a vertical strut.

3) Develop and validate the release of water droplets in the RIBC. 
4) Release water droplets into the spray bar simulations with and without a vertical strut.

5) Alter the current spray bar geometry to recreate the "dancing" phenomenon without the need of a vertical strut.

6) Consider other tunnel speeds and spray jet pressures 


\section{Appendix A}

Images of FLUENT to execute the methods described previously:

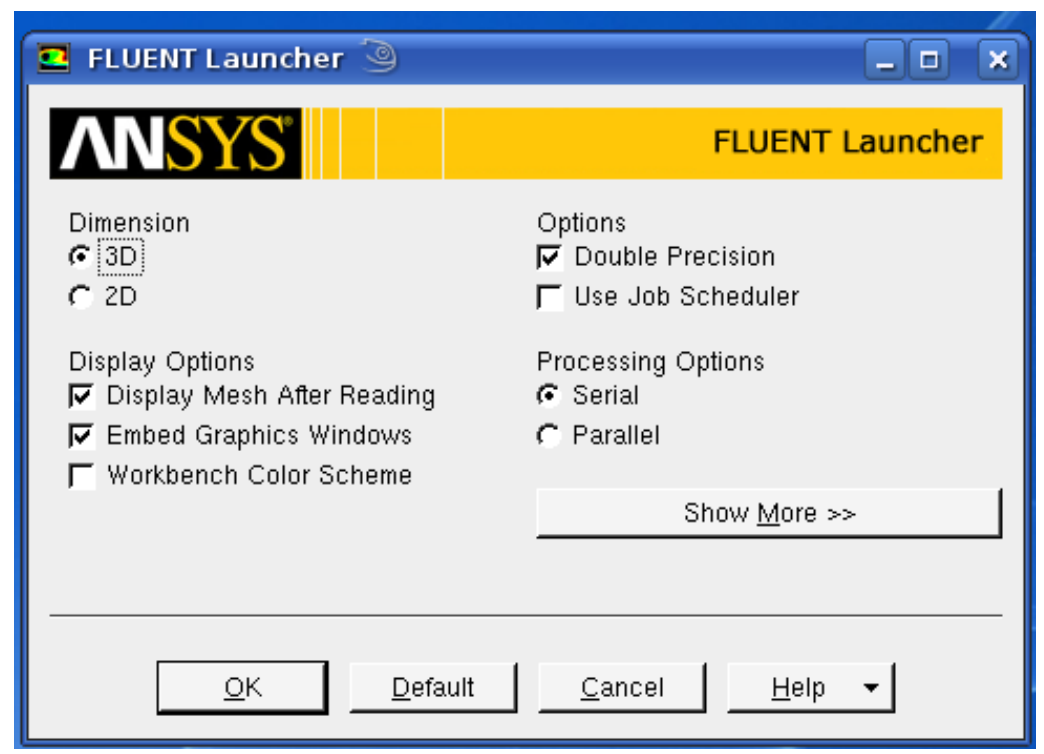

Figure A.1: Launch window for FLUENT which selects the dimension, precision and number of computer processors. Double precision was used for all cases while dimension (2D-3D) and number of process vary (serial - 12 parallel).

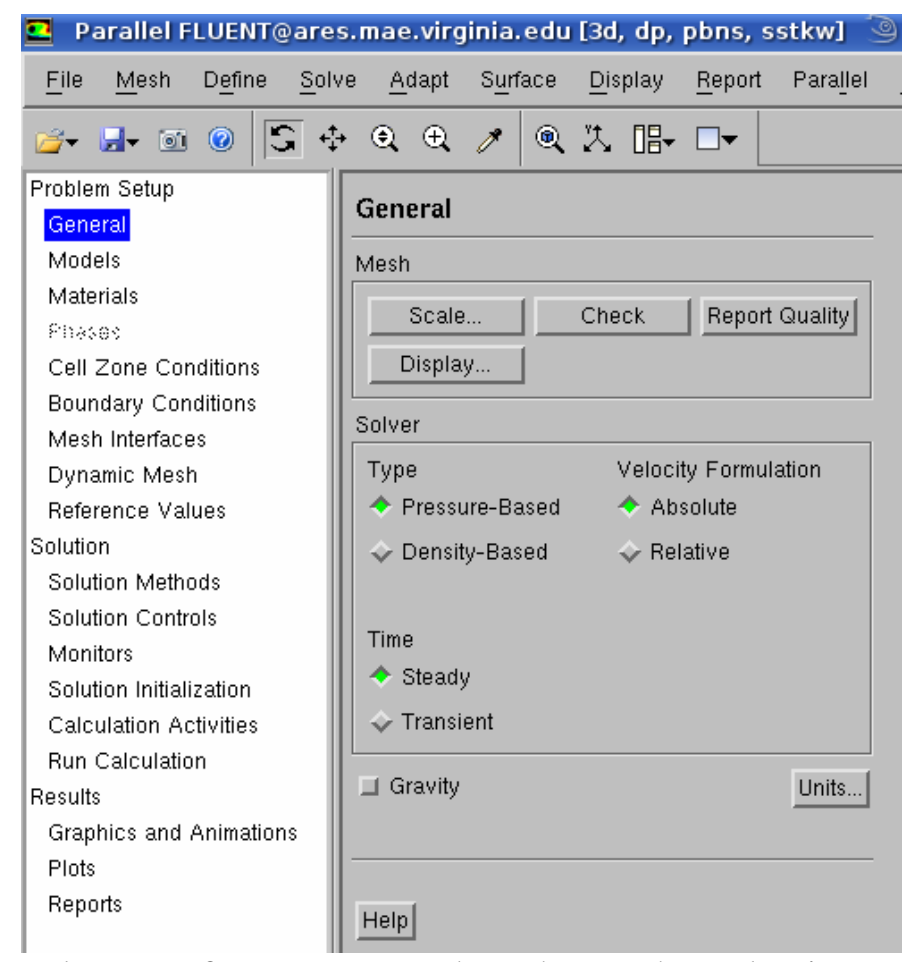

Figure A.2: General menu for FLUENT that shows the selection of a pressure-based solver and steady-state solution used in all tunnel configuration simulations. 


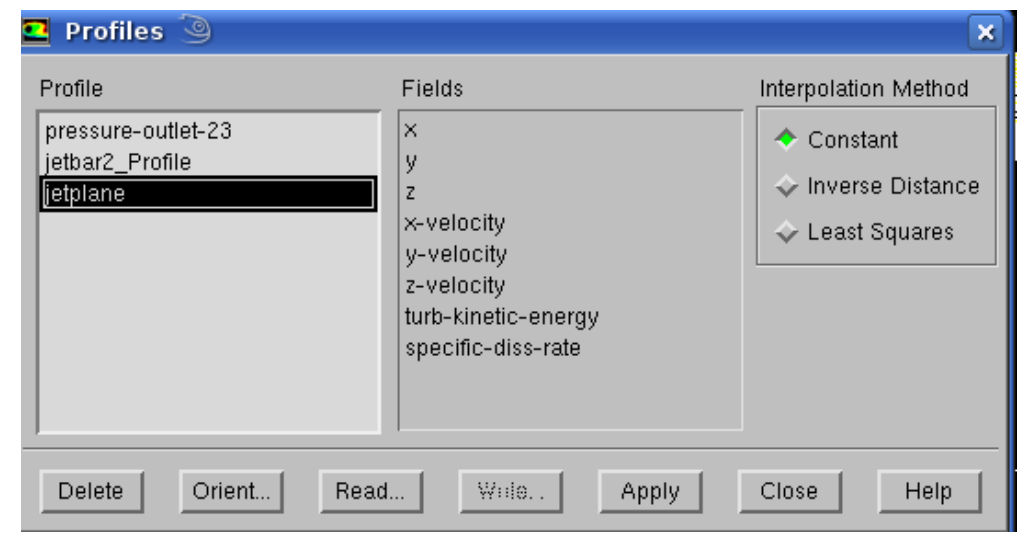

Figure A.3: Photo of the profile screen needed to read profiles used for the 4" jet profile and inlet boundary profiles in different sections of the tunnel.

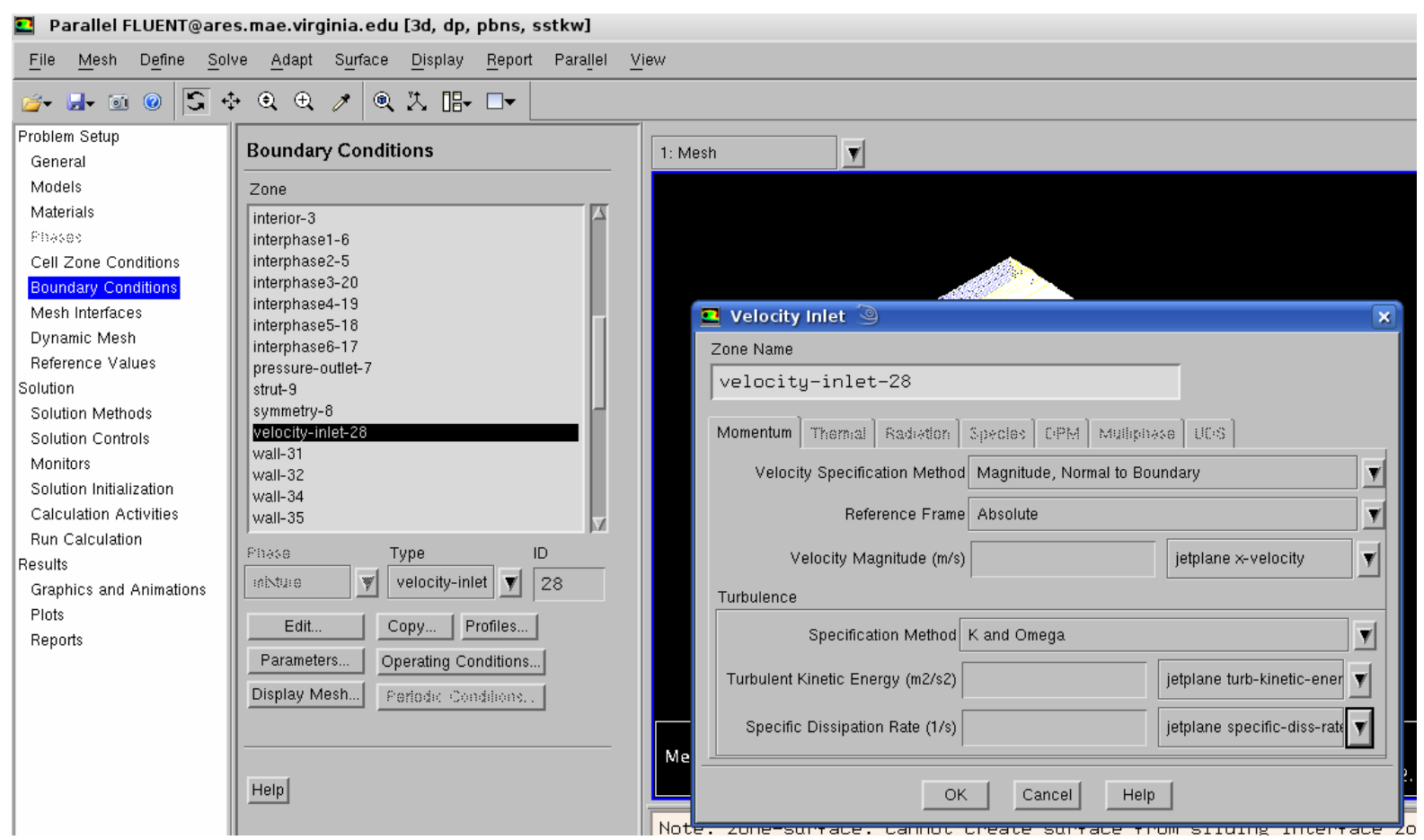

Figure A.4: The boundary conditions menu and a window for a velocity inlet set to a profile that was previously read into FLUENT. The boundary condition menu is used to set all boundary conditions and set interphases to be meshed together. 


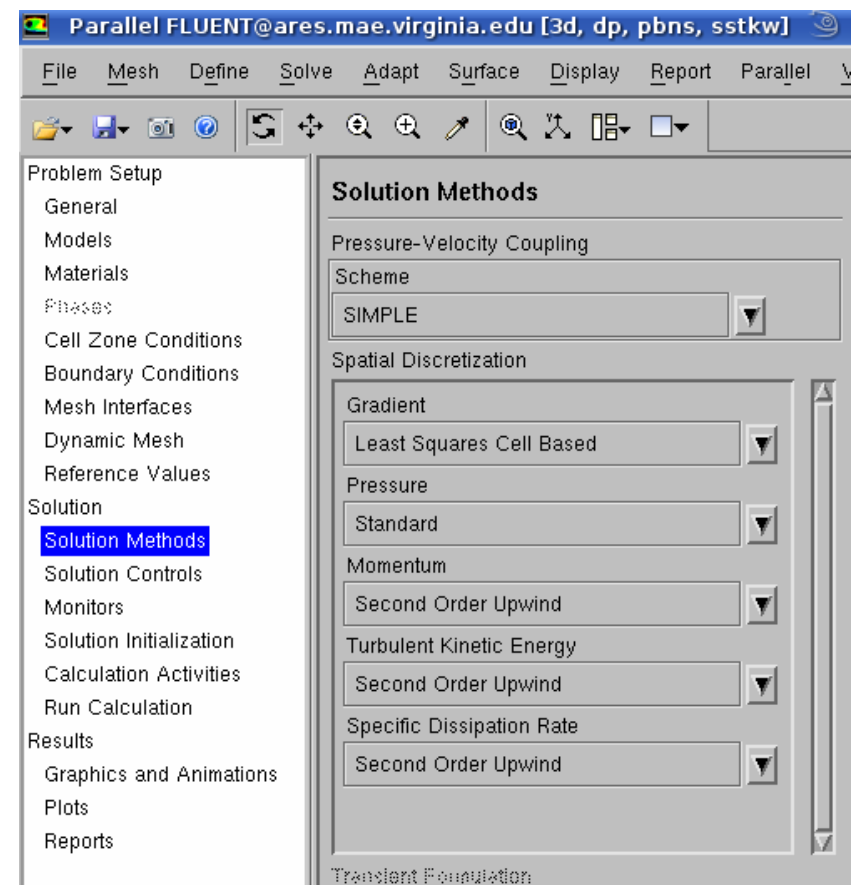

Figure A.5: The methods menu displaying the selections for the SIMPLE pressurevelocity coupling and second-order upwind methods.

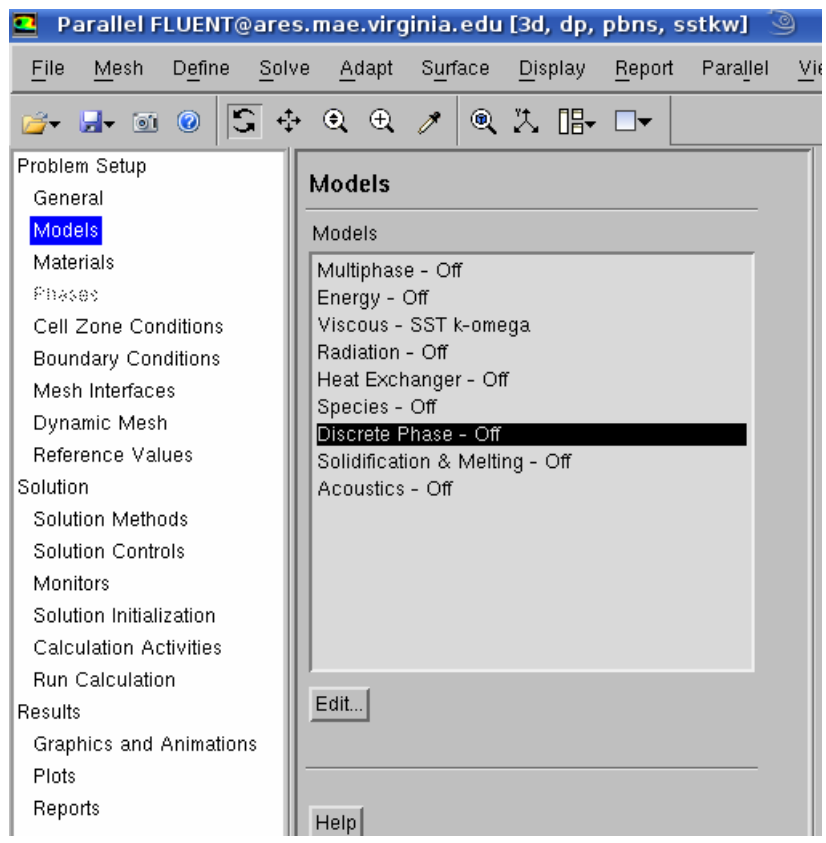

Figure A.6: The model menu to turn on the discrete phase models that execute the Lagrangain calculations for the water droplet trajectories. 


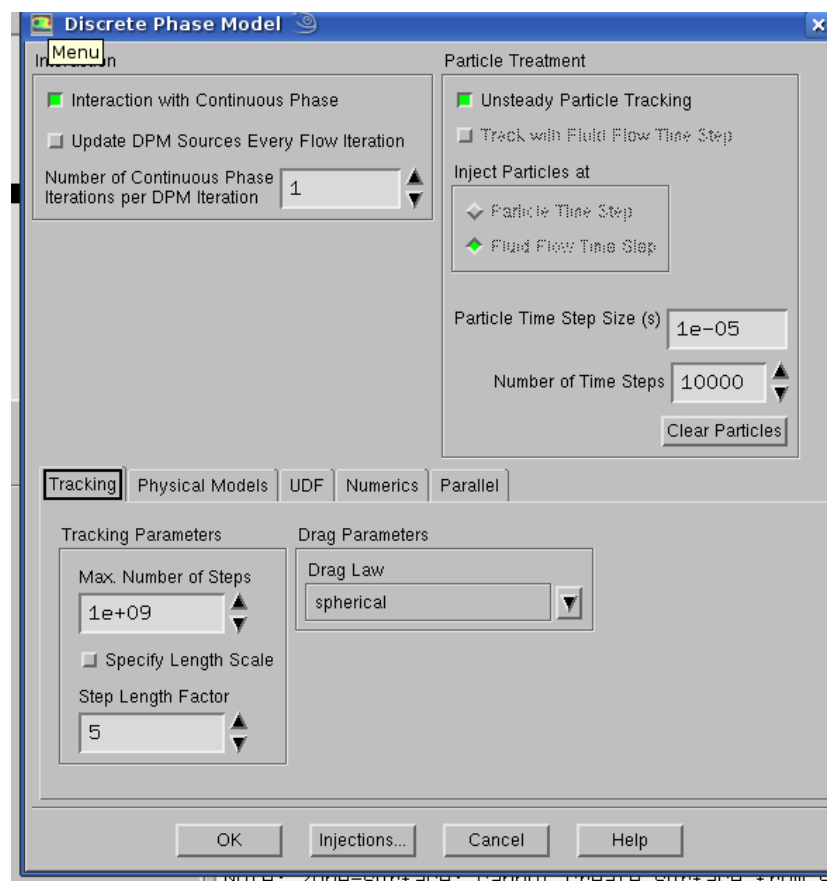

Figure A.7: The discrete phase model menu with unsteady particle tracking active for the water droplet trajectories.

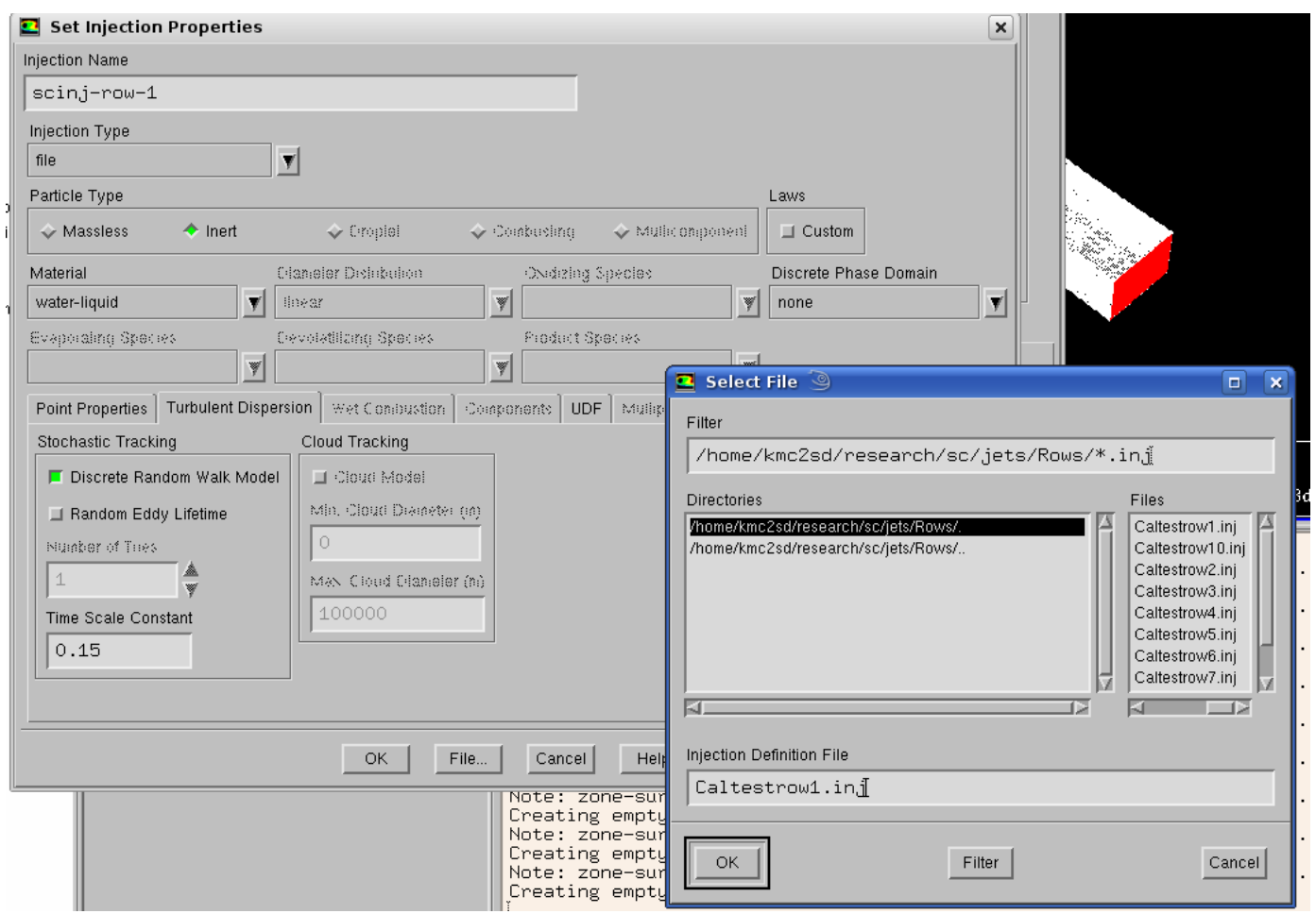

Figure A.8: The injection widows that are using the file type of injection. This allows for the input of injection setting to be imported through an existing file. The DRW model is also activated in this menu. 


\section{Appendix B}

Here is an example of the input file that WIND requires (single spray bar simulation):

IRT Spraybar, 3-D, 3 zones

$\mathrm{V}=175 \mathrm{mph}, \mathrm{D}=19.406 \mathrm{in}$

$\mathrm{Re}=227425$ (reference length=D)

Freestream static 0.0172860737 14.695950254 459.67 0. 0.

Downstream pressure 14.70103 zone 3

Turbulence SST

hybrid VERSION 3 DELTA 2 zone all

!FREE_K 0.527595

!FREE_OM 3.73543

max_wall_distance 35 grid_units

!MULTIPHASE

!file irtspry.phz

!END MULTIPHASE

RHS ROE FIFTH UPWINDBIASED

COUPLING ROE LOW

!TVD OFF zone all

DQ LIMITER ON DTMAX 0.2

Implicit full full full zone all

timestep seconds $0.4 \mathrm{E}-6$

!cfl 0.5 zone all

Cycles 40000

Iteration per cycle 1 zone 1

Iteration per cycle 1 zone 2

Iteration per cycle 1 zone 3

Iteration per cycle 1 zone 4

!SEQUENCE 111 zone 1

!SEQUENCE 111 zone 2

!SEQUENCE 111 zone 3

!SEQUENCE 111 zone 4

arbitrary inflow

static 
zone 4

ijk_range frozen $11114511370.017286073714 .695950254459 .67 \quad 0.0 .0 .527595$ 3.73643

end inflow

SPAWN " /save_cfl /research/irtspry/walls/irtspry.cfl" FREQUENCY 10000 


\section{References}

${ }^{1}$ Gonsalez j., Arrington A., and Curry M., "Flow Quality Surveys of the NASA Glenn Icing Research Tunnel (2000 Tests)," $39^{\text {th }}$ AIAA Aerospace Sciences Meeting \& Exhibit, Reno, NV, AIAA-2001-0232

${ }^{2}$ Ide, R. F., and Oldenburg, J. R., "Icing Cloud Calibration of the NASA Glenn Icing Research Tunnel,” AIAA Paper 2001-0234, Jan. 2001.

${ }^{3}$ Hancir, P., Anderson, A., and Loth, E., "Computations of Droplet Distributions in the NASA Icing Research Tunnel," $38^{\text {th }}$ AIAA Aerospace Sciences Meeting, Reno, NV, Jan. 2000, AIAA Paper 2000-0101.

${ }^{4}$ Bhargava, C., Loth, E., Potapczuk, M., "Numerical Simulation of Icing Clouds in the NASA Glenn Icing Research Tunnel" $42^{\text {nd }}$ AIAA Aerospace Sciences Meeting, Reno, NV, Jan. 2004, AIAA Paper 2004-563.

${ }^{5}$ Anderson, D., Tannehill, J., and Pletcher, R., Computational Fluid Mechanics and Heat Transfer, McGraw-Hill, New York, 1984, pp. 199-206.

${ }^{6}$ Menter, F., "Two-Equation Eddy-Viscosity Turbulence Models for Engineering Applications," AIAA Journal, Vol. 32, No. 8, August 1994, pp. 1598-1605.

${ }^{7}$ Snyder, W. H., and Lumley, J. L., "Some Measurements of Particle Velocity Autocorrelation Functions in a Turbulent Flow" Journal of Fluid Mechanics, Vol. 48, Part. 1, 1971, pp. 41-71

${ }^{8}$ Anderson, J. Computational Fluid Dynamics, McGraw-Hill International Editions, New York, 1995, pp. 247-262.

${ }^{9}$ Bulzan, D.L., Levy, Y., Aggarwal, S.K., and Chitre, S., "Measurements and Predictions of a Liquid Spray from an Air-Assist Nozzle," AIAA 29th Aerospace Sciences Meeting, Reno, NV, AIAA Paper 1991-692.

${ }^{10}$ Lee, A., and Loth, E., "Droplet Dispersion in the NASA Icing Research Tunnel," AIAA Atmospheric Space Environments Conference, Toronto, ON, Aug 2010, AIAA2010-7533.

${ }^{11}$ Loth, E., Particles, Drops and Bubbles: Fluid Dynamics and Numerical Methods, Cambridge University Press, 2013.

${ }^{12}$ Van Zante, J. F., Ide, R. F., Steen, L. E., "NASA Glenn Icing Research Tunnel: 2011 Cloud Calibration Procedure and Results", AIAA Atmospheric Sciences Meeting, New Orleans, Jun. 2012

${ }^{13}$ Irvine, T. B., Oldenburg, J. R., and Sheldon, D. W., "New Icing Cloud Simulation System at the NASA Glenn Research Center Icing Research Tunnel," 1999, NASA TM-1999-208891. 
${ }^{14}$ Steen, L. E., Van Zante, J. F., Broeren, A., Kubiak, M. "Flow Quality Surveys in the Settling Chamber of the NASA Glenn Icing Research Tunnel (2011 Tests)", AIAA Atmospheric Sciences Meeting, New Orleans, Jun. 2012

${ }^{15}$ Crowe, C., Sommerfeld, M., Tsuji Y., Multiphase Flows with Droplets and Particles, CRC Press, Boston, 1998, pp. 195-197

${ }^{16}$ Morsi, S.A., and Alexander, A.J., "An Investigation of Particle Trajectories in TwoPhase Flow Systems" Journal of Fluid Mechanics, Vol. 55, Part 2, 1972, pp. 193208.

${ }^{17}$ Muldoon F., and Acharya S., "Direct Numerical Simulation of pulsed jets-incrossflow," Computers \& Fluids 39, 2010, pp. 1745-1773.

${ }^{18}$ Cai J., Tsai H., and Liu F., "Numerical simulation of vertical flows in the near field of jets from notched circular nozzles," Computers \& Fluids 39, 2010, pp. 539-552.

${ }^{19}$ Dinesh K.K.J., Savill A.M., Jenkins K.W., and Kirkpatrick M.P., "LES of intermittency in a turbulent round jet with different inlet conditions," Computer \& Fluids 39, 2010, pp. 1685-1695.

${ }^{20}$ Urbin, G., and Knight, D., "Large Eddy Simulation of a Supersonic Boundary Layer Using an Unstructured Grid," AIAA Journal, Vol.39, No.7, July 2001.

${ }^{21}$ Yuan, L., Street, R., and Ferziger, J., "Large-eddy simulations of a round jet in crossflow" 1999 Cambridge University Press

${ }^{22}$ Ferziger, J., Peric, M., Computational Methods for Fluid Dynamics, Springer, Berlin, 1999, pp.268-272

${ }^{23}$ Nichols, R. H., and Nelson, C. C., "Application of Hybrid RANS/LES Turbulence Models,” AIAA Paper 2003-0083, Jan. 2003.

${ }^{24}$ Brigham, E., The Fast Fourier Transform, Prentice-Hall, Inc., N.J., 1974, pp.148-150

${ }^{25}$ Dong, S., Karniadakis, G., Ekmekci, A., Rockwell, D., "A combined direct numerical simulation-particle image velocimetry study of the turbulent near wake," J. Fluid Mech, Vol. 569, 2006, pp. 185-207 